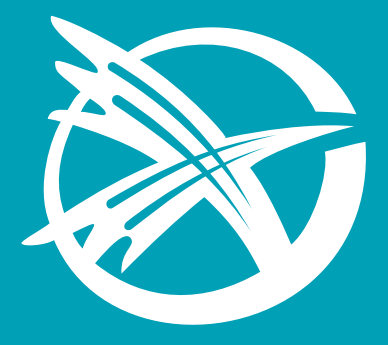

\title{
BULLETIN $(\xi-a)$
}

OF THE KARAGANDA UNIVERSITY
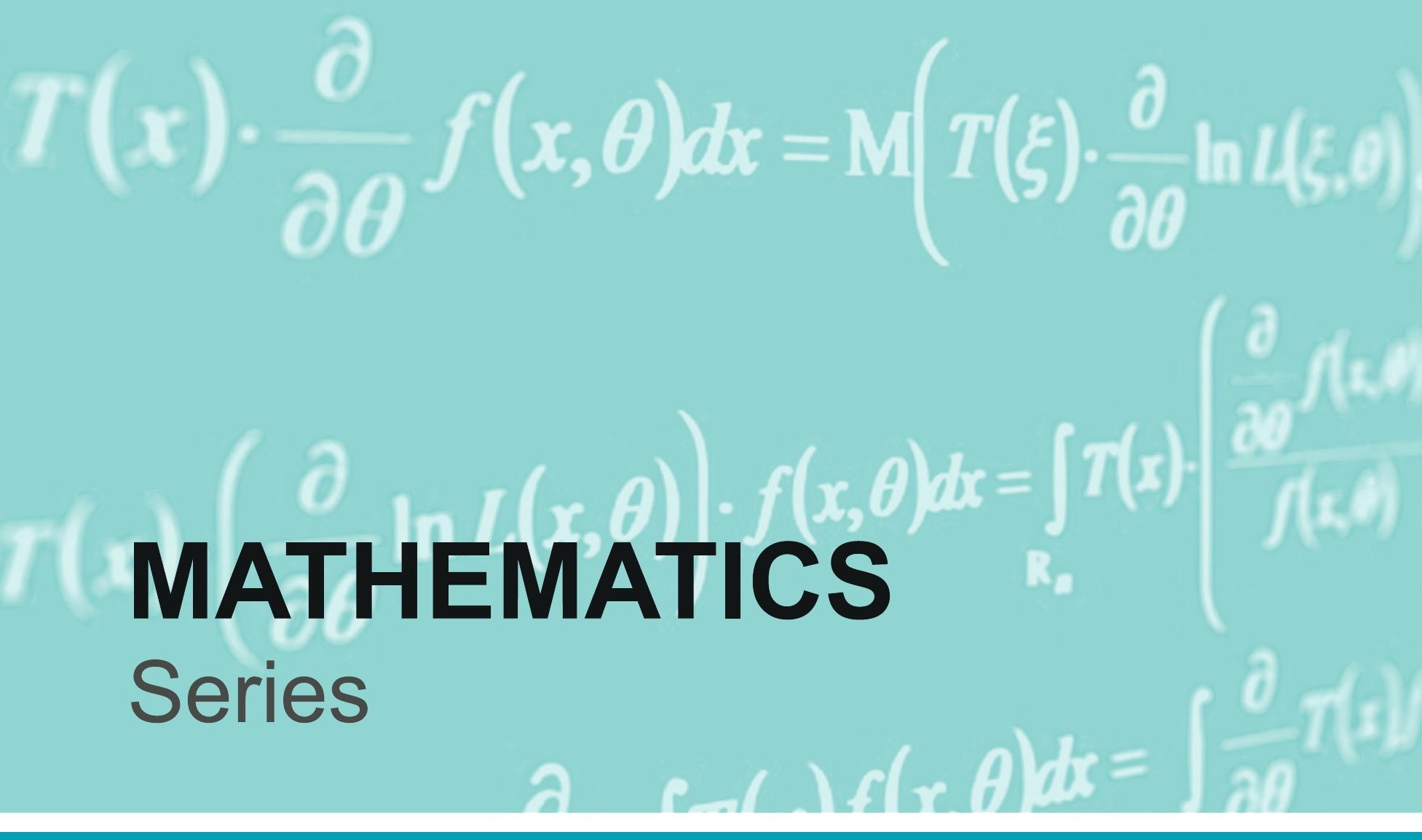

№ 1(101)/2021 
ISSN 2518-7929 (Print)

ISSN 2663-5011(Online)

Индексі 74618

Индекс 74618

\section{ҚАРАҒАНДЫ \\ УНИВЕРСИТЕТІНІН \\ ХАБАРШЫСЫ}

\section{ВЕСТНИК \\ КАРАГАНДИНСКОГО \\ УНИВЕРСИТЕТА}

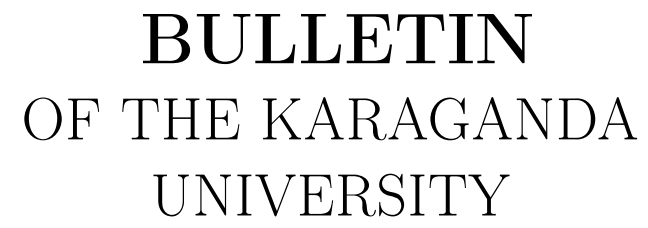

МАТЕМАТИКА сериясы

Серия МАТЕМАТИКА

MATHEMATICS Series

№ $1(101) / 2021$

Қаңтар-ақпан-наурыз

30 наурыз 2021 ж.

Январь-февраль-март

30 марта 2021 г.

January-February-March

March 30 ${ }^{\text {th }}, 2021$

1996 жылдан бастап шығады

Издается с 1996 года

Founded in 1996

Жылына 4 рет шығады

Выходит 4 раза в год

Published 4 times a year

Қарағанды, 2021

Караганда, 2021

Karaganda 2021 


\title{
Main Editor \\ Candidate of Physics and Mathematics sciences \\ N.T. Orumbayeva
}

\author{
Responsible secretary \\ $\mathrm{PhD}$ \\ O.I. Ulbrikht
}

\section{Editorial board}

\section{Otelbayev, \\ B.R. Rakishev, M.A. Sadybekov, \\ U.U. Umirbaev, \\ T. Bekjan, \\ B Poizat,}

A.A. Shkalikov,

A.S. Morozov,

A. Ashyralyev,

G. Akishev,

A.T. Assanova,

N.A. Bokaev,

M.T. Jenaliyev,

A.R. Yeshkeyev,

K.T. Iskakov,

L.K. Kusainova,

E.D. Nursultanov,

M.I. Ramazanov,

E.S. Smailov,

M.T. Kosmakova,
Academician NAS RK, Dr. phys.-math. sciences, Gumilyov ENU, Nur-Sultan (Kazakhstan);

Academician of NAS RK, Dr. techn. sciences, Satbayev University, Almaty (Kazakhstan); Corresponding member of NAS RK, Dr. phys.-math. sciences, IMMM, Almaty (Kazakhstan);

Corresponding member of NAS RK, Dr. phys.-math. sciences, L.N. Gumilyov ENU, Nur-Sultan (Kazakhstan);

Professor, Xinjiang University, Urumchi (China);

Professor, Universite Claude Bernard Lyon-1, Villeurbanne (France);

Corresponding member of RAS RF, Dr. phys.-math. sciences, Lomonosov Moscow State University, Moscow (Russia);

Dr. phys.-math. sciences, Sobolev Institute of Mathematics, Novosibirsk (Russia);

Dr. phys.-math. sciences, Near East University, Nicosia, TRNC, Mersin 10 (Turkey);

Dr. phys.-math. sciences, Gumilyov ENU, Nur-Sultan (Kazakhstan);

Dr. phys.-math. sciences, IMMM, Almaty (Kazakhstan);

Dr. phys.-math. sciences, Gumilyov ENU, Nur-Sultan (Kazakhstan);

Dr. phys.-math. sciences, IMMM, Almaty (Kazakhstan);

Dr. phys.-math. sciences, Buketov KU, Karaganda (Kazakhstan);

Dr. phys.-math. sciences, Gumilyov ENU, Nur-Sultan (Kazakhstan);

Dr. phys.-math. sciences, Gumilyov ENU, Nur-Sultan (Kazakhstan);

Dr. phys.-math. sciences, KB Lomonosov MSU, Nur-Sultan (Kazakhstan);

Dr. phys.-math. sciences, Buketov KU, Karaganda (Kazakhstan);

Dr. phys.-math. sciences, IMMM, Almaty (Kazakhstan);

$\mathrm{PhD}$, Buketov KU, Karaganda (Kazakhstan)

Postal address: 28, University Str., Karaganda, 100024, Kazakhstan

Tel.: (7212) 77-04-38 (add. 1026); fax: (7212) 35-63-98.

E-mail: vestnikku@gmail.com. Web-site: mathematics-vestnik.ksu.kz

\section{Editors}

Zh.T. Nurmukhanova, S.S. Balkeyeva

Computer layout

G.K. Kalel

Bulletin of the Karaganda University. Mathematics series.

ISSN 2518-7929 (Print). ISSN 2663-5011 (Online).

Proprietary: NLC «Karagandy University of the name of academician E.A. Buketov».

Registered by the Ministry of Information and Social Development of the Republic of Kazakhstan.

Rediscount certificate No. KZ43VPY00027385 dated 30.09.2020.

Signed in print 29.03.2021. Format $60 \times 84$ 1/8. Offset paper. Volume 18,75 p.sh. Circulation 200 copies. Price upon request. Order № 14.

Printed in the Publishing house of NLC «Karagandy University of the name of acad. E.A. Buketov». 28, University Str., Karaganda, 100024, Kazakhstan. Tel.: (7212) 35-63-16. E-mail: izd_kargu@mail.ru 


\section{CONTENTS}

\section{MATHEMATICS}

Attaev A.Kh. On Problem of Internal Boundary Control for String Vibration Equation $\ldots \ldots \ldots \ldots \ldots \quad 4$

Bekbolat B., Tokmagambetov N. Well-posedness results for the wave equation generated by the Bessel

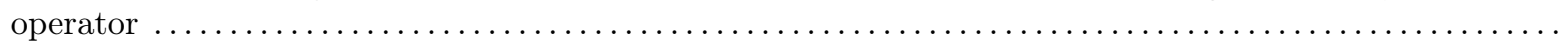

Bokayev N.A., Khairkulova A.A. On the boundedness of the fractional maximal operator on global

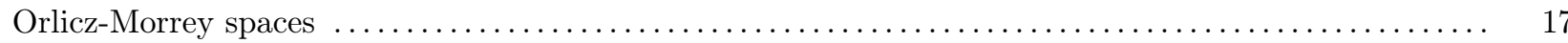

Davoodi S.M., Abdul Rahman N.A. Predicting the optimal solution in fuzzy linear programming problem

Jenaliyev M.T., Ramazanov M.I., Tanin A.O. To the solution of the Solonnikov-Fasano problem with boundary moving on arbitrary law

Jwamer K. H. F., Ahmed Sh. Sh., Abdullah D.Kh. Approximate Solution of Volterra Integro-Fractional

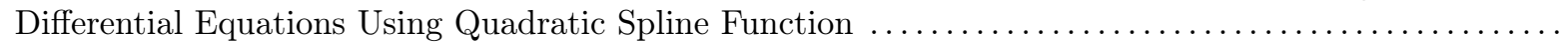

Kosmakova M.T., Iskakov, S.A., Kasymova L.Zh. To solving the fractionally loaded heat equation ...

Kvasnikov V.P., Yehorov S.V., Shkvarnytska T.Yu. Technology for restoring functional dependencies to determine reliability parameters

Muratbekov I.B., Suleimbekova A.O. On the existence of the resolvent and separability of a class of the Korteweg-de Vriese type linear singular operators

Tleubergenov M.I., Vassilina G.K., Tuzelbaeva G.A. On construction of a field of forces along given

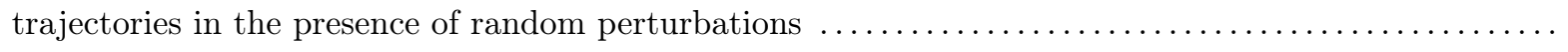

Yesbayev A.N., Ospanov M.N. The solvability conditions for the second order nonlinear differential

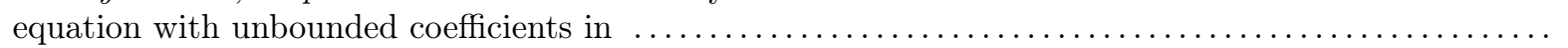

Yeshkeyev A.R., Mussina N.M. An algebra of the central types of the mutually model-consistent frag-

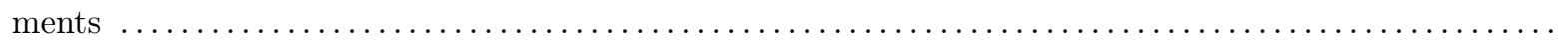

Yeshkeyev A.R., Omarova M.T. An essential base of the central types of the convex theory .........

Yuldashev T.K., Islomov B.I., Ubaydullaev U.Sh. On Boundary Value Problems for a Mixed Type Frac-

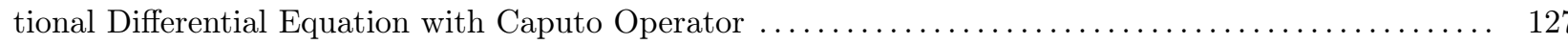

Yuldashev T.K., Zarifzoda S.K. On a New Class of Singular Integro-differential Equations ......... 138

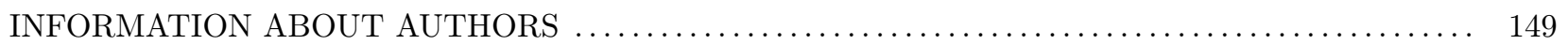




\title{
MATЕМАТИКА MATHEMATICS
}

DOI $10.31489 / 2021 \mathrm{M} 1 / 4-10$

UDC 517.956

\author{
A.Kh. Attaev \\ Institute of Applied Mathematics and Automation of Kabardin-Balkar Scientific Centre of RAS, Nalchik, Russia \\ (E-mail: attaev.anatoly@yandex.ru)
}

\section{On Problem of Internal Boundary Control for String Vibration Equation}

\begin{abstract}
The article deals with the vibration control problem described by one dimensional wave equation with integral type boundary condition. As usual, the initial and final moments of time for arbitrary displacements and velocities of the wave are specified by points on a string (Cauchy data). It is shown that the minimum time for the realizable control is uniquely determined by the condition of correct solvability to the Cauchy problem involving data lying on disconnected manifold. This suggests that the internal boundary conditions does not affect the minimum time value. Necessary and sufficient conditions for the existence of the desired internal-boundary controls that move the process from the state initially specified to a predetermined final one are obtained and written out. The controls are presented in explicit analytical form. Moreover, it is shown that for the inner-boundary controls expressions, one should use not the representation of the solution to the Cauchy problem in the sought-for domain, but the formula for the general solution of the string oscillation equation (d'Alembert's formula).
\end{abstract}

Keywords: string vibration equation, boundary control, Cauchy problem, trunk and branched pipeline networks, nonlocal mixed problem.

\section{Introduction}

One of the main parts of systems with distributed parameters in the Control Theory is one-dimensional distributed-parameter system for objects with the motion described by hyperbolic partial differential equations. As a rule, these objects control requires considering oscillation and wave propagation. We refer to objects include such technical facilities as a compressor and pumping stations providing distributing water via trunk and branched pipeline networks at a given flow rate and pressure. The problem of pressure pulsation dampening in pipelines is considered to be a classical one. A detailed account on engineering aspects and mathematical formulations for this problem and also various solution techniques can be found in [1], as well as in [2,3]. There are many works devoted to boundary control problems for hyperbolic equations, including loaded ones, and to optimization problems in terms of an arbitrary sufficiently large time interval, and others, we here mention only some of them [4-14]. These papers investigated boundary control problems for hyperbolic equations with both local and nonlocal multi-point boundary conditions. The boundary control problem is formulated as follows: Define the control action for moving the internal state of a system from any initial state to any other final state in a finite time interval. If the controls are determined on the boundary such problem is called the boundary control problem. If controls are defined inside the domain as well as on the boundary such a problem is logically called the problem of the internal boundary control. In mathematical terms, the unique solvability of the control problem is equivalent to the well-posed solvability of the Cauchy problem with data lying on a disconnected 
manifold. It is this fact that makes it possible to determine the minimum time during which unambiguous control is carried out.

The following results were obtained:

1. Necessary and sufficient conditions $\varphi_{0}(x), \varphi_{1}(x), \psi_{0}(x), \psi_{1}(x), k_{1}(x, t), k_{2}(x, t)$, are established ensuring the existence of the boundary controls $\mu(t)$ and $\nu(t)$, in the form of

$$
\begin{gathered}
\varphi_{0}^{\prime}(0)-\psi_{0}(0)-\varphi_{1}^{\prime}(l)+\psi_{1}(l)=0, \\
\varphi_{0}^{\prime}(l)+\psi_{0}(l)-\varphi_{1}^{\prime}(0)-\psi_{1}(0)=0, \\
\varphi_{0}^{\prime \prime}(0)-\psi_{0}^{\prime}(0)-\varphi_{1}^{\prime \prime}(l)+\psi_{1}^{\prime}(l)=0, \\
\varphi_{0}^{\prime \prime}(l)+\psi_{0}^{\prime}(l)-\varphi_{1}^{\prime \prime}(0)-\psi_{1}^{\prime}(0)=0, \\
\varphi_{0}(0)+\psi_{0}(l)+\int_{0}^{l} \psi_{0}(\xi) d \xi-\varphi_{1}(0)-\varphi_{1}(l)+\int_{0}^{l} \psi_{1}(\xi) d \xi=0, \\
\int_{0}^{l} k_{i}(\xi, 0) \varphi_{0}(\xi) d \xi=0, \int_{0}^{l} k_{i}(\xi, l) \varphi_{1}(\xi) d \xi=0, \quad i=1,2, \\
\int_{0}^{l} k_{i}^{\prime}(\xi, 0) \varphi_{0}(\xi) d \xi+\int_{0}^{l} k_{i}(\xi, 0) \psi_{0}(\xi) d \xi=0, \quad i=1,2, \\
\int_{0}^{l} k_{i}^{\prime}(\xi, l) \varphi_{0}(\xi) d \xi+\int_{0}^{l} k_{i}(\xi, l) \psi_{1}(\xi) d \xi=0, \quad i=1,2 .
\end{gathered}
$$

2. Under conditions (1)-(4), an explicit analytical form of the sought controls is found

$$
\begin{aligned}
& \mu(t)=\frac{1}{2} \varphi_{0}(t)+\frac{1}{2} \int_{0}^{t} \psi_{0}(\xi) d \xi+\frac{1}{2} \varphi_{1}(l-t)+\frac{1}{2} \int_{l-t}^{l} \psi_{1}(\xi) d \xi+\frac{1}{2} \varphi_{0}(0)-\frac{1}{2} \varphi_{1}(l)+U_{k_{1}}(t), \\
& \nu(t)=\frac{1}{2} \varphi_{0}(l-t)+\frac{1}{2} \int_{l-t}^{l} \psi_{0}(\xi) d \xi+\frac{1}{2} \varphi_{1}(t)+\frac{1}{2} \int_{0}^{t} \psi_{1}(\xi) d \xi+\frac{1}{2} \varphi_{0}(l)-\frac{1}{2} \varphi_{1}(0)+U_{k_{2}}(t),
\end{aligned}
$$

where

$$
\begin{gathered}
U_{k_{i}}(t)=\frac{1}{2} \int_{0}^{l-t} k_{i}(t+\xi, t) \varphi_{0}(\xi) d \xi+\frac{1}{2} \int_{t}^{l} k_{i}(\xi-t, t) \varphi_{0}(\xi) d \xi+ \\
\frac{1}{2} \int_{t}^{l} k_{i}(\xi-t, t) d \xi \int_{0}^{t} \psi_{0}(\xi) d \xi-\frac{1}{2} \int_{0}^{l-t}\left(\int_{\xi}^{l-t} k_{i}(t+\eta, t) d \eta\right) \psi_{0}(\xi) d \xi+ \\
\frac{1}{2} \int_{t}^{l}\left(\int_{\xi}^{l} k_{i}(\eta-t, t) d \eta\right) \psi_{0}(\xi) d \xi+\frac{1}{2} \int_{0}^{t} k_{i}(\xi+l-t, t) \varphi_{1}(\xi) d \xi+ \\
\frac{1}{2} \int_{0}^{t} k_{i}(\xi+l-t, t) d \xi \int_{t}^{l} \psi_{1}(\xi) d \xi+\frac{1}{2} \int_{l}^{l-t}\left(\int_{l-\xi}^{t} k_{i}(t-\eta, t) d \eta\right) \psi_{1}(\xi) d \xi+ \\
\frac{1}{2} \int_{0}^{t}\left(\int_{0}^{\xi} k_{i}(\eta+l-t, t) d \eta\right) \psi_{1}(\xi) d \xi+\frac{1}{2}\left[\varphi_{0}(0)-\varphi_{1}(l)\right]\left[\int_{0}^{t} k_{i}(z, t) d z-\int_{l-t}^{l} k_{i}(z, t) d z\right], i=1,2 .
\end{gathered}
$$

Below the problem of internal-boundary-value control for the string oscillation equation is formulated and the possibility for obtaining unique solution in minimum time interval using the control and the Cauchy problems is discussed. 


\section{Main part}

In the domain $\Omega=\{(x, t): 0<x<l, 0<t<T\}$ the one-dimensional equation of string vibration is considered

$$
u_{x x}-u_{t t}=0,
$$

describing, for example, the string vibration with the ends fixed at the points $x=0$ and $x=l$. Assume that arbitrary displacements and arbitrary speeds are set at the initial time $t=0$ and the final time $t=T$ :

$$
\begin{gathered}
u(x, 0)=\varphi_{0}(x), u_{t}(x, 0)=\psi_{0}(x), 0 \leq x \leq l, \\
u(x, T)=\varphi_{1}(x), u_{t}(x, T)=\psi_{1}(x), 0 \leq x \leq l .
\end{gathered}
$$

Internal boundary conditions are specified by the following relations:

$$
\begin{aligned}
& u(0, t)+\int_{0}^{l} k_{1}(\xi, t) u(\xi, t) d \xi=\mu(t), \\
& u(l, t)+\int_{0}^{2} k_{2}(\xi, t) u(\xi, t) d \xi=\nu(t),
\end{aligned}
$$

where $k_{1}(x, t), k_{2}(x, t)$ - are the given functions, and $k_{i}(x, t), \frac{\partial}{\partial t} k_{i}(x, t) \in C(\bar{\Omega}), i=1,2$.

Further in this paper the function $u(x, t) \in C^{2}(\Omega) \cap C^{1}(\bar{\Omega})$ - is understood as the solution to equation (11). The problem is to find such values of $\mu(t)$ and $\nu(t)$ for unambiguous moving the system from state (14) to state (15) in the minimum time interval.

From the problem formulated above it immediately follows that $\mu(t)$ and $\nu(t)$ are uniquely determined in the domain $\Omega$ if and only if the Cauchy problem (12), (13) for equation (11) in the domain $\Omega$ is well-posed.

Indeed, in case when the Cauchy problem (12), (13) for equation (11) occurs underdetermined, the infinite set of $(\mu(t), \nu(t))$ can perform the desired control. This implies the non-uniqueness of the solution to the Cauchy problem (12), (13) for equation (11) in the domain $\Omega$. If the Cauchy problem (12), (13) for equation (11) in the domain $\Omega$ is underdetermined, then control is possible only for linearly depend (12) and (13). These items are discussed in [4] and in more detail in [14]. There you can also find out that the Cauchy problem (12), (13) for equation (11) in the domain $\Omega$ is well-posed if and only if the value of $T$ is equal to $l$. If $T>l$, the Cauchy problem is underdetermined. IF $T<l$, the Cauchy problem is redefined. This suggests that the desired control is unambiguously feasible if and only if $T=l$.

Consider $T=l$. It is well known that any regular solution to equation (11) can be represented as follows:

$$
u(x, t)=f(x-t)+g(x+t),
$$

where $f(x)$ and $g(x)$ - $\mathrm{g}(\mathrm{x})$ - are arbitrary twice-continuously differentiable functions. Further, for conveniences for the functions $f(x)$ and $g(x)$ from formula (16), introduce the following notation:

$$
\begin{gathered}
f(x)=f_{0}(x), g(x)=g_{0}(x), x \in[0, l], \\
f(x)=f_{1}(x), x \in[-l, 0], \\
g(x)=g_{1}(x), x \in[l, 2 l] .
\end{gathered}
$$

Satisfying conditions (12) for (16) and using the notation, obtain

$$
\begin{aligned}
& f_{0}(x)=\frac{\varphi_{0}(x)}{2}-\frac{1}{2} \int_{0}^{x} \psi_{0}(\xi) d \xi+C_{1}, \\
& g_{0}(x)=\frac{\varphi_{0}(x)}{2}+\frac{1}{2} \int_{0}^{x} \psi_{0}(\xi) d \xi-C_{1} .
\end{aligned}
$$

Now satisfying conditions (13) for (16), as $T=l$, we obtain

$$
\begin{gathered}
f(x-l)+g(x+l)=\varphi_{1}(x), \\
-f^{\prime}(x-l)+g^{\prime}(x+l)=\psi_{1}(x) .
\end{gathered}
$$


Hence, it is obvious that

$$
f^{\prime}(x-l)=\frac{\varphi_{1}^{\prime}(x)}{2}-\frac{\psi(x)}{2} .
$$

Using the last identity integration from $l$ to $l-x$, get

$$
f(-x)=\frac{\varphi_{1}^{\prime}(l-x)}{2}-\frac{\varphi_{1}^{\prime}(l)}{2}-\frac{1}{2} \int_{l}^{l-x} \psi_{1}(\xi) d \xi+f(0) .
$$

Taking into account that $f_{0}(0)=\frac{\varphi_{0}(0)}{2}+C_{1}$ by (17) and using our notation, obtain

$$
f_{1}(-x)=\frac{\varphi_{1}(l-x)}{2}+\frac{1}{2} \int_{l-x}^{l} \psi_{1}(\xi) d \xi+\frac{\varphi_{0}(0)}{2}+\frac{\varphi_{1}(l)}{2}+C_{1}, x \in[0, l],
$$

and by (13) get

$$
g_{1}(l+x)=\frac{\varphi_{1}(x)}{2}-\frac{1}{2} \int_{x}^{l} \psi_{1}(\xi) d \xi-\frac{\varphi_{0}(0)}{2}+\frac{\varphi_{1}(l)}{2}-C_{1}, x \in[0, l] .
$$

Now let us find the control actions $\mu(t)$ and $\nu(t)$. Generally speaking, the solution to the Cauchy problem in the domain $\Omega$ as $T=l$ could be found and the resulting expression could be substituted into (14) and (15) respectively. We find another way that does not require solving the Cauchy problem.

Substituting (16) sequentially (13) and (14), we obtain

$$
\begin{gathered}
\mu(t)=V_{0}(t)+V_{k_{1}}(t), \\
\nu(t)=V_{l}(t)+V_{k_{2}}(t), \\
V_{k_{i}}(t)=\int_{0}^{l} k_{i}(\xi, t) f(\xi-t) d \xi+\int_{0}^{l} k_{i}(\xi, t) g(\xi+t) d \xi, \\
V_{0}(t)=f_{1}(-t)+g_{0}(t), V_{l}(t)=f_{0}(l-t)+g_{1}(l+t), t \in[0, l] .
\end{gathered}
$$

Replacing $t-\xi=z$ in the first integral of (23), and $t+\xi=l+z$ in the second, we obtain

$$
\begin{aligned}
V_{k_{i}}(t) & =\int_{t-l}^{t} k_{i}(t-z, t) f(z) d z+\int_{t-l}^{t} k_{i}(z+l-t, t) g(l+z) d z= \\
= & \int_{0}^{t} k_{i}(t-z, t) f(z) d z+\int_{0}^{t} k_{i}(z+l-t, t) g(l+z) d z+ \\
+ & \int_{t-l}^{0} k_{i}(t-z, t) f(z) d z+\int_{t-l}^{0} k_{i}(z+l-t, t) g(l+z) d z .
\end{aligned}
$$

Substituting $-z=z$ and $l+z=z$ in the last two integrals, respectively, and using our notation for the functions $f(x)$ and $g(x)$, finally obtain the expression for $V_{k_{i}}(t)$.

$$
\begin{gathered}
V_{k_{i}}(t)=\int_{0}^{t} k_{i}(t-z, t) f_{1}(-z) d z+\int_{0}^{t} k_{i}(z+l-t, t) g_{1}(l+z) d z+ \\
\quad+\int_{0}^{l-t} k_{i}(t+z, t) f_{0}(z) d z+\int_{t}^{l} k_{i}(z-t, t) g_{0}(z) d z, i=1,2 .
\end{gathered}
$$

Replace $f_{0}, g_{0}, f_{1}, g_{1}$ with expressions from (17), (18) and (19), (20), substitute then the expressions into $(21)$ and (22) for $V_{k_{1}}(t)$ and $V_{k_{2}}(t)$, respectively, and making some transformations, obtain (9), (10).

Since the functions $u(x, t)$ belong to the class $C^{2}(\Omega) \cap C^{1}(\bar{\Omega})$, the continuity conditions

and consistency conditions

$$
\begin{gathered}
f_{0}(0)=f_{1}(0), f_{0}^{\prime}(0)=f_{1}^{\prime}(0), f_{0}^{\prime \prime}(0)=f_{1}^{\prime \prime}(0), \\
g_{0}(l)=g_{1}(l), g_{0}^{\prime}(l)=g_{1}^{\prime}(l), g_{0}^{\prime \prime}(l)=g_{1}^{\prime \prime}(l)
\end{gathered}
$$

$$
\begin{gathered}
\mu(0)=\varphi_{0}(0), \mu(l)=\varphi_{1}(0), \mu^{\prime}(0)=\psi_{0}(0), \mu^{\prime}(l)=\psi_{1}(0), \\
\nu(0)=\varphi_{0}(l), \nu(l)=\varphi_{1}(l), \nu^{\prime}(0)=\psi_{0}(l), \nu^{\prime}(l)=\psi_{1}(l) .
\end{gathered}
$$


Conditions (24), (25) are necessary and sufficient for the existence of a solution to the mixed problem (12), (13) for equation (11) in the domain $\Omega$ as $T=l$, in the form of

$$
u(x, t)=f_{i}(x-t)+g_{j}(x+t),-i l<x-t<(1-i) l, j l<x+t<(1+j) l, i, j=0,1 .
$$

Conditions (24)-(27) are necessary and sufficient for the functions $\varphi_{0}(x), \varphi_{1}(x) \in C^{2}(0, l) \cap C^{1}[0, l], \psi_{0}(x)$, $\psi_{1}(x) \in C^{1}[0, l]$, there were internal boundary controls $\mu(t), \nu(t) \in C^{2}(0, l) \cap C^{1}[0, l]$ satisfying conditions (13) solutions $u(x, t) \in C^{2}(\Omega) \cap C^{1}(\bar{\Omega})$ to the mixed problem (12) for equation (11).

The proof of the uniqueness of the solution to the mixed problem (12), (13) and nonlocal mixed problems (12), (14), (15) and (13), (14), (15) for equation (11) is carried out the same way as in [4]. Substituting (17) and (18) into (24) and (25), respectively, and (14) and (15), into (26) and (27), we obtain (1)-(8).

\section{References}

1 Бутковский А.Г. Теория оптимального управления системами с распределенными параметрами / А.Г. Бутковский - М.: Наука, 1965. - 476 с.

2 Bayraktar B. Determining optimum hydraulic parameters in branched water supply system / B. Bayraktar // Journal of the Balkan Tribological Assocition. - 2012. - 18. - № 2. - P. 174-183.

3 Bayraktar B. Problem of Constructing a Step Function Fluctuating Least around a Given Function / B. Bayraktar // TWMS Journal of Pure and Applied Mathematics. - 2013. - 4. - № 2. - P. 131-145.

4 Ильин В.А. Волновое уравнение с граничным управлением на двух концах и о полном успокоении колебательного процесса / В.А. Ильин, В.В. Тихомиров // Дифференц. уравнения. $-1999 .-35$. - № 5. - С. 692-704.

5 Ильин В.А. Граничное управление смещением на одном конце струны при наличии нелокального граничного условия одного из четырех типов и его оптимизация / В.А. Ильин // Дифференц. уравнения. - 2008. - 44. - № 11. - С. 1487-1498.

6 Моисеев Е.И. Оптимальное граничное управление смещенными колебаниями струны с нелокальным условием нечетности первого рода / Е.И. Моисеев, А.А. Холомеева // Дифференц. уравнения. 2010. - 46. - № 11. - С. 1623-1630.

7 Моисеев Е.И. Оптимальное граничное управление смещенными колебаниями струны с нелокальным условием четности второго рода / Е.И. Моисеев, А.А. Холомеева // Дифференц. уравнения. - 2011. - 47. - № 1. - C. 127-134.

8 Attaev A.Kh. Boundary control by displacement at one end of string and the integral condition on the other // Int. Conf. "Functional Analysis in Interdisciplinary Applications"(FAIA 2017), Melville, New York: Am. Inst. Phys. - 2017. - P. 040005.

9 Аттаев А.Х. Задача граничного управления для существенно нагруженного уравнения колебания струны / А.Х. Аттаев // Докл. Адыгской (Черкесской) Междунар. акад. наук. - 2011. - 13. - № 1. - C. $30-35$.

10 Бутковский А.Г. Оптимальное управление двумерной колебательной системой / А.Г. Бутковский, Л.И. Полтавский // Автоматика и телемеханика. - 1966. - № 4. - С. 32-41.

11 Lions J.L. Exact controllability, stabilization, and perturbations for distributed systems / Lions J. L. // SIAM Rev. - 1988. - 30. - № 2. - P. 1-68.

12 Васильев Ф.П. О двойственности в линейных задачах управления и наблюдения / Ф.П. Васильев // Дифференц. уравнения. - 1995. - 31. - № 11. - С. 1893-1900.

13 Бутковский А.Г. Методы управления системами с распределенными параметрами / А.Г. Бутковский. - М.: Наука, 1975. - 568 с.

14 Attaev A.Kh. Boundary control problem for a loaded string vibration equation / A.Kh. Attaev // Differ. Equations. - 2020. - 56. - 5. - P. 635-640. 


\title{
А.Х. Аттаев \\ Ішек тербелісінің теңдеуі үшін ішкі-шеттік басқарудың бір есебі жайында
}

\begin{abstract}
Мақалада интегралды типті ішкі-шеттік шарттары бар бір өлшемді ішектің тербеліс теңдеуімен сипатталатын процесті басқару есебі қарастырылған. Әдеттегідей, уақыттың бастапқы және соңғы сәттерінде еркін ығысулар мен ішек нүктелерінің жылдамдығы (Коши мәліметтері) беріледі. Ізделінді басқарудың жалғыз мүмкін болатын ең аз уақыты шекаралық үзілісті көпбейнеліктерімен Коши есебінің корректілі шешілу жағдайынан нақты анықталатындығы көрсетілген. Бұл ішкі-шеттік шарттардың түрі минималды уақыт мәніне әсер етпейтінін көрсетеді. Тербелмелі жүйені бастапқы берілген күйден алдын-ала берілген соңғы күйге аударатын ішкі-шеттік басқармалардың қажетті және жеткілікті жағдайлары алынып жазылды. Басқармалардың өздері нақты аналитикалық түрде жазылған. Сонымен қатар, ішкі-шеттік басқару үшін өрнектерді алуда ізделінді облыстағы Коши есебін шешімімен емес, ішек тербеліс теңдеуінің жалпы шешімінің формуласын (Даламбер формуласы) қолдану керек екендігі көрсетілген.
\end{abstract}

Kiлm сөздер: ішектің тербеліс теңдеуі, шекаралық басқару есебі, Коши есебі, магистральдық және тармақталған құбыр желілері, бейлокалды аралас есеп.

\section{A.X. Аттаев \\ Об одной задаче внутренне-краевого управления для уравнения колебания струны}

В статье рассмотрена задача управления процессом, который описывается уравнением колебания одномерной струны с внутренне-краевыми условиями интегрального типа. Как обычно, в начальный и финальный моменты времени задаются произвольные смещения и скорости точек струны (данные Коши). Показано, что минимальное время, в течение которого единственным образом осуществимо искомое управление однозначным образом, определяется из условия корректной разрешимости задачи Коши с данными на граничном разрывном многообразии. Это свидетельствует о том, что сам вид внутренне-краевых условий на значение минимального времени не влияет. Получены и выписаны необходимые и достаточные условия существования искомых внутренне-краевых управлений, переводящих колебательную систему из начально заданного состояния в наперед заданное финальное состояние. Сами управления выписаны в явном аналитическом виде. При этом показано, что для получения выражений для внутренне-краевых управлений нужно воспользоваться не самим представлением решения задачи Коши в искомой области, а формулой общего решения уравнения колебания струны (формулой Даламбера).

Ключевые слова: уравнение колебания струны, задача граничного управления, задача Коши, магистральные и разветвленные трубопроводные сети, нелокальная смешанная задача.

\section{References}

1 Butkovskij, A.G. (1965). Teoriia optimalnoho upravleniia sistemami s raspredelennymi parametrami /The theory of optimal control of systems with distributed parameters]. Moscow: Nauka [in Russian].

2 Bayraktar, B. (2012). Determining optimum hydraulic parameters in branched water supply system. Journal of the Balkan Tribological Assocition, 18(2), 174-183.

3 Bayraktar, B. (2013). Problem of Constructing a Step Function Fluctuating Least around a Given Function. TWMS Journal of Pure and Applied Mathematics, 4(2), 131-145.

4 Il'in, V.A., \& Tihomirov, V.V. (1999). Volnovoe uravnenie s hranichnym upravleniem na dvukh kontsakh i o polnom uspokoenii kolebatelnoho protsessa [The wave equation with boundary control at two ends and the problem of the complete damping of a vibration process]. Differentsialnye uravneniia - Differ. Equations, 35(5), 692-704 [in Russian]. 
5 Il'in, V.A. (2008). Hranichnoe upravlenie smeshcheniem na odnom kontse struny pri nalichii nelokalnoho hranichnoho usloviia odnoho iz chetyrekh tipov i eho optimizatsiia [Boundary control by displacement at one end of a string in the presence of a nonlocal boundary condition of one of four types and its optimization]. Differentsialnye uravneniia - Differ. Equations, 44(11), 1487-1498 [in Russian].

6 Moiseev, E.I., \& Holomeeva, A.A. (2010). Optimalnoe hranichnoe upravlenie smeshchennymi kolebaniiami struny s nelokalnym usloviem nechetnosti pervoho roda [Optimal boundary displacement control of string vibrations with a nonlocal oddness condition of the first kind]. Differentsialnye uravneniia - Differ. Equations, 46(11), 1623-1630 [in Russian].

7 Moiseev, E.I., \& Holomeeva, A.A. (2011). Optimalnoe hranichnoe upravlenie smeshchennymi kolebaniiami struny s nelokalnym usloviem chetnosti vtoroho roda [Optimal boundary displacement control of string vibrations with a nonlocal evenness condition of the second kind]. Differentsialnye uravneniia - Differ. Equations, 47(1), 127-134 [in Russian].

8 Attaev, A.Kh. (2017). Boundary control by displacement at one end of string and the integral condition on the other. Int. Conf. "Functional Analysis in Interdisciplinary Applications"(FAIA 2017), Melville, New York: Am. Inst. Phys., 040005.

9 Attaev, A.Kh. (2011). Zadacha hranichnoho upravleniia dlia sushchestvenno-nahruzhennoho uravneniia kolebaniia struny [Boundary control problem for essentially loaded string vibration equation]. Doklady Adyhskoi (Cherkesskoi) Mezhdunarodnoi akademii nauk, 13(1), 30-35 [in Russian].

10 Butkovskij, A.G., \& Poltavskij, L.I. (1966). Optimalnoe upravlenie dvumernoi kolebatelnoi sistemoi [Optimal control over a two-dimensional vibrational system ]. Avtomatika i telemekhanika, 4, 32-41 [in Russian].

11 Lions, J. L. (1988). Exact controllability, stabilization, and perturbations for distributed systems. SIAM Rev, 30(2), 1-68.

12 Vasil'ev, F.P. (1995). O dvoistvennosti v lineinykh zadachakh upravleniia i nabliudeniia [On duality in linear control and observation problems]. Differentsialnye uravneniia - Differ. Equations, 31(11), 18931900 [in Russian].

13 Butkovskij, A.G. (1975). Metody upravleniia sistemami s raspredelennymi parametrami [Methods for control systems with distributed parameters]. Moscow: Nauka [in Russian].

14 Attaev, A.Kh. (2020). Boundary control problem for a loaded string vibration equation. Differ. Equations, $56(5), 635-640$. 


\author{
B. Bekbolat ${ }^{1,2,3}{ }^{*}$, N. Tokmagambetov ${ }^{1,2,3}$ \\ ${ }^{1}$ Al-Farabi Kazakh National University, Almaty, Kazakhstan; \\ ${ }^{2}$ Institute of Mathematics and Mathematical Modeling, Almaty, Kazakhstan; \\ ${ }^{3}$ Department of Mathematics: Analysis, Logic and Discrete Mathematics, Ghent University, Belgium \\ (E-mail: bekbolat@math.kz,niyaz.tokmagambetov@gmail.com)
}

\title{
Well-posedness results for the wave equation generated by the Bessel operator
}

\begin{abstract}
In this paper, we consider the non-homogeneous wave equation generated by the Bessel operator. We prove the existence and uniqueness of the solution of the non-homogeneous wave equation generated by the Bessel operator. The representation of the solution is given. We obtained a priori estimates in Sobolev type space. This problem was firstly considered in the work of M. Assal [1] in the setting of Bessel-Kingman hypergroups. The technique used in [1] is based on the convolution theorem and related estimates. Here, we use a different approach. We study the problem from the point of the Sobolev spaces. Namely, the conventional Hankel transform and Parseval formula are widely applied by taking into account that between the Hankel transformation and the Bessel differential operator there is a commutation formula [2].
\end{abstract}

Keywords: Bessel operator, wave equation, Hankel transform, inverse Hankel transform, Sobolev type space.

\section{Introduction}

In this paper we consider the nonhomogeneous wave equation

$$
\frac{\partial^{2} u}{\partial t^{2}}-S_{\mu} u=f(x, t), \quad x \in R_{+}, \quad 0<t<T
$$

under the conditions

$$
u(x, 0)=u_{0}(x), u_{t}(x, 0)=u_{1}(x),
$$

where $T$ is fixed positive real number, generated by the Bessel operator

$$
S_{\mu} \triangleq \frac{d^{2}}{d x^{2}}+\frac{1-4 \mu^{2}}{4 x^{2}}
$$

where $\mu \geq-\frac{1}{2}$. The operator (1) is widely analysed in [2]. In the book [2], the author considered the Hankel transform in the countably multinormed space $\mathcal{H}$. Between the Hankel transform and (1) there is a commutation formula. The heat equation generated by (1) was studied in [3]. In [3], it was proved that the Cauchy problem has a unique solution $u(x, t)$ in the space $\left(U_{\mu, q^{\prime}, 1 /(b+d)}^{q^{\prime}, 1 /(a-d)}\right)^{\prime}, q^{\prime}=\left(2 p_{0}-1\right) /\left(2 p_{0}\right)$, for the interval $0 \leq t \leq T$, $T<\left(4 c p_{0}\right)^{-1}(d / 2)^{2 p_{0}}, d<a$. Where $\left(U_{\mu, q^{\prime}, 1 /(b+d)}^{q^{\prime}, 1 /(a-d)}\right)^{\prime}$ is dual for $U_{\mu, q^{\prime}, 1 /(b+d)}^{q^{\prime}, 1 /(a-d)}$. Here $U_{\mu, q^{\prime}, 1 /(b+d)}^{q^{\prime}, 1 /(a-d)}$ is a linear space (see [3]), where the topology over this space is generated by the norm

$$
\|f\|_{\delta \rho}=\sup _{s \in \mathbb{C}}\left|s^{-\mu-\frac{1}{2}} f(s)\right| \cdot \exp \left(q^{\prime}\left[\left(\frac{1}{b+d}-\delta\right) x\right]-q^{\prime}\left[\left(\frac{1}{a-d}+\rho\right) y\right]\right) .
$$

A generalized Bessel operator is given by the following expression

$$
\frac{d^{2}}{d x^{2}}+\frac{2 \mu+1}{x} \frac{d}{d x}
$$

In [1] the author studied the operator (2) in the setting of Bessel-Kingman hypergroups and as an application studied the homogeneous wave equation. In the paper [1] it is studied radial solutions of the Cauchy problem for the wave equations in the multidimensional unit ball $B^{d}, d \geq 1$. For more information about the analysis associated with the generalized Bessel operator, we refer to [4-10].

\footnotetext{
${ }^{*}$ Corresponding author.

E-mail: bekbolat@math.kz
} 


\section{The test function space}

The space $\mathcal{H}_{\mu}$ is defined for each $\mu \in \mathbb{R}$ as follows (see [2]):

Definition 1. $\mathcal{H}_{\mu}$ is a space of complex-valued, smooth functions and for each pair of $m, k \in \mathbb{Z}_{+}$with the seminorms

$$
\gamma_{m, k}^{\mu}(f) \triangleq \sup _{0<x<\infty}\left|x^{m}\left(x^{-1} D\right)^{k}\left(x^{-\mu-\frac{1}{2}} f(x)\right)\right|<\infty .
$$

Lemma 1. $\mathcal{H}_{\mu}$ is complete. Thus, it is a Fréchet space.

We denote by $\mathcal{H}_{\mu}^{\prime}$ the dual of $\mathcal{H}_{\mu}$. $\mathcal{H}_{\mu}^{\prime}$ is also complete.

We introduce following linear operators:

$$
\begin{gathered}
N_{\mu} f(x) \triangleq x^{\mu+\frac{1}{2}} D x^{-\mu-\frac{1}{2}} f(x), \\
M_{\mu} f(x) \triangleq x^{-\mu-\frac{1}{2}} D x^{\mu+\frac{1}{2}} f(x), \\
N_{\mu}^{-1} f(x) \triangleq x^{\mu+\frac{1}{2}} \int_{\infty}^{x} t^{-\mu-\frac{1}{2}} f(t) d t .
\end{gathered}
$$

Now, we can rewrite (1) as follows

$$
S_{\mu}=M_{\mu} N_{\mu}=\frac{d^{2}}{d x^{2}}+\frac{1-4 \mu^{2}}{4 x^{2}} .
$$

$S_{\mu}$ is a continuous linear mapping as

$$
S_{\mu}: \mathcal{H}_{\mu} \rightarrow \mathcal{H}_{\mu} .
$$

\section{The Hankel transform and its inverse}

If $\mu \geq-\frac{1}{2}$, for every $\phi \in \mathcal{H}_{\mu}$ we can define the conventional Hankel transform

$$
\Phi(y)=\left(h_{\mu} \phi\right)(y)=\int_{0}^{\infty} \sqrt{x y} J_{\mu}(x y) \phi(x) d x, \quad 0<y<\infty .
$$

Here, the kernel $\sqrt{x y} J_{\mu}(x y)$ is an eigenfunctions of the operator $S_{\mu}(1)$.

Lemma 2. ([2]) Assume that $\mu \geq-\frac{1}{2}$ and $f \in \mathcal{H}_{\mu}$. Then

$$
h_{\mu}\left(S_{\mu} f\right)=-y^{2} h_{\mu} f .
$$

Theorem 1. ([11]) Let $f \in L^{1}$. If $f$ is a bounded variation in a neighborhood of the point $x=x_{0}$, if $\mu \geq-\frac{1}{2}$, and if $F(y)$ is defined by (3), then

$$
\frac{1}{2}\left[f\left(x_{0}\right)+f\left(x_{0}-0\right)\right]=h_{\mu}^{-1} F=\int_{0}^{\infty} F(y) \sqrt{x_{0} y} J_{\mu}\left(x_{0} y\right) d y .
$$

Theorem 2. ([2]) The Hankel transformation $h_{\mu}$ is an automorphism on $\mathcal{H}_{\mu}$, for $\mu \geq-\frac{1}{2}$.

Theorem 3. ([2]) Let $\mu \geq-\frac{1}{2}$. If $f(x)$ and $G(y)$ are in $L^{1}(0, \infty)$ then

$$
\int_{0}^{\infty} f(x)\left(h_{\mu}^{-1} G\right)(x) d x=\int_{0}^{\infty} F(y) G(y) d y .
$$

Definition 2. ([12]) For $s, \mu \in \mathbb{R}$ and $1 \leq p<\infty$ the Sobolev type space $G_{\mu}^{s, p}$ is the set of all tempered distributions $u \in \mathcal{H}^{\prime}$ such that $U$ is locally integrable function over $I:=(0, \infty)$ and

$$
\|u\|_{G_{\mu}^{s, p}}^{p}:=\int_{0}^{\infty}\left(1+y^{2}\right)^{s p}|U(y)|^{p} d y<\infty .
$$

In view of the Parseval formula

$$
\|u\|_{2}^{2}=\|U\|_{2}^{2}, \quad \mu \geq-\frac{1}{2}
$$


it follows that

$$
G_{\mu}^{0,2}(I)=L^{2}(I)
$$

Theorem 4. ([12]) The Sobolev type space $G_{\mu}^{s, p}, 1 \leq p<\infty$, is complete.

Theorem 5. ([12]) $\mathcal{H}_{\mu}$ is dense in $G_{\mu}^{s, p}, 1 \leq p<\infty, \forall s \in \mathbb{R}$.

\section{Wave equation generated by the Bessel operator}

In this section, we consider the Cauchy problem for the nonhomogeneous wave equation generated by the Bessel operator on the $Q_{T}:=\left\{(t, x): t \in[0, T], x \in \mathbb{R}_{+}\right\}$. Our aim to prove that the Problem 1 has a unique solution in the space $C^{2}\left([0, T], L^{2}(I)\right) \cap C\left([0, T], G_{\mu}^{1,2}(I)\right)$.

Problem 1. Let $T$ be a fixed positive number. We aim to find a function $u=u(t, x)$ satisfying the nonhomogeneous wave equation

$$
u_{t t}(t, x)-S_{\mu} u(t, x)=f(t, x), \quad(t, x) \in Q_{T}
$$

under the conditions

$$
\begin{gathered}
u(0, x)=u_{0}(x), \\
u_{t}(0, x)=u_{1}(x) .
\end{gathered}
$$

The Problem 1 has solution in the space $C^{2}\left([0, T], L^{2}(I)\right) \cap C\left([0, T], G_{\mu}^{1,2}(I)\right)$ and as a result we obtain following theorem:

Theorem 6. Let $\mu \geq-1 / 2$ and assume that $f \in C\left([0, T], G_{\mu}^{1,2}(I)\right)$ and $u_{0}, u_{1} \in G_{\mu}^{1,2}(I)$. Then the Problem 1 has a unique solution $u$ and it can be represented by the expression

$$
\begin{gathered}
u(t, x)=\int_{0}^{\infty} \sqrt{x y} J_{\mu}(x y) \int_{0}^{t} F(\tau, y) \frac{\sin y(t-\tau)}{y} d \tau d y+ \\
\int_{0}^{\infty} \sqrt{x y} J_{\mu}(x y) U_{1}(y) \frac{\sin (y t)}{y} d y+\int_{0}^{\infty} \sqrt{x y} J_{\mu}(x y) U_{0}(y) \cos (y t) d y
\end{gathered}
$$

where $F(\tau, y), U_{0}(y)$ and $U_{1}(y)$ are Hankel transforms of the functions $f(t, x), u_{0}(x)$ and $u_{1}(x)$, respectively.

Proof. By using the Hankel transform, we can show the uniqueness of the solution if the later exists. First, we prove the existence of the solution. After using the Hankel transform $h_{\mu}(3)$ and (4) for (7)-(9), we obtain

$$
\begin{gathered}
U_{t t}(t, y)+y^{2} U(t, y)=F(t, y), \quad y \in R_{+}, \\
U(0, y)=U_{0}(y), \quad y \in R_{+} \\
U_{t}(0, y)=U_{1}(y), \quad y \in R_{+} .
\end{gathered}
$$

Solution of the equation (10) is

$$
\begin{gathered}
U(t, y)=\left(\int_{0}^{t} \frac{F(\tau, y)}{y} \cos (y \tau) d \tau+A(y)\right) \sin (y t)+ \\
\left(-\int_{0}^{t} \frac{F(\tau, y)}{y} \sin (y \tau) d \tau+B(y)\right) \cos (y t) .
\end{gathered}
$$

By using initial conditions (11)-(12) for (13), we have

$$
U(t, y)=\int_{0}^{t} F(\tau, y) \frac{\sin y(t-\tau)}{y} d \tau+U_{1}(y) \frac{\sin (y t)}{y}+U_{0}(y) \cos (y t)
$$

After using the inverse Henkel transform $h_{\mu}^{-1}(5)$, we obtain that the solution of the problem (7)-(9) is given by

$$
\begin{gathered}
u(t, x)=\int_{0}^{\infty} \sqrt{x y} J_{\mu}(x y) \int_{0}^{t} F(\tau, y) \frac{\sin y(t-\tau)}{y} d \tau d y+ \\
\int_{0}^{\infty} \sqrt{x y} J_{\mu}(x y)\left(U_{1}(y) \frac{\sin (y t)}{y} d y+U_{0}(y) \cos (y t)\right) d y
\end{gathered}
$$

Mathematics series. № 1(101)/2021 
$F \lesssim G$ denotes $F \leq C G$ for some positive constant $C$ independent of $F$ and $G$.

Let $f \in C\left([0, T], G_{\mu}^{1,2}(I)\right)$ and $u_{0}, u_{1} \in G_{\mu}^{1,2}(I)$. By taking into account the definition of the space $G_{\mu}^{1,2}(I)$ (Definition 2), we obtain

$$
\begin{gathered}
\|u(t, \cdot)\|_{G_{\mu}^{1,2}}^{2}=\left\|\left(1+y^{2}\right) U(t, \cdot)\right\|_{2}^{2}=\int_{0}^{\infty}\left|\left(1+y^{2}\right) U(t, y)\right|^{2} d y \\
\lesssim \int_{0}^{\infty}\left|\left(1+y^{2}\right) \int_{0}^{t} F(\tau, y) \frac{\sin y(t-\tau)}{y} d \tau\right|^{2} d y+\int_{0}^{\infty}\left|\left(1+y^{2}\right) U_{1}(y) \frac{\sin (y t)}{y}\right|^{2} d y \\
+\int_{0}^{\infty}\left|\left(1+y^{2}\right) U_{0}(y) \cos (y t)\right|^{2} d y .
\end{gathered}
$$

Now, we are going to do some necessary calculations, as following

$$
\begin{gathered}
\int_{0}^{\infty}\left|\left(1+y^{2}\right) \int_{0}^{t} F(\tau, y) \frac{\sin y(t-\tau)}{y} d \tau\right|^{2} d y \\
=\int_{0}^{\infty}\left|\left(1+y^{2}\right) \int_{0}^{t} F(\tau, y) \frac{\sin y(t-\tau)}{y(t-\tau)} \cdot(t-\tau) d \tau\right|^{2} d y \lesssim \int_{0}^{\infty}\left(\left(1+y^{2}\right) \int_{0}^{t}|F(\tau, y)| d \tau\right)^{2} d y \\
\lesssim \int_{0}^{\infty}\left(1+y^{2}\right)^{2}\left(\int_{0}^{T}|F(t, y)| d t\right)^{2} d y \lesssim \int_{0}^{\infty} \int_{0}^{T}\left|\left(1+y^{2}\right) F(t, y)\right|^{2} d t d y=\int_{0}^{T}\|f(t, \cdot)\|_{G_{\mu}^{1,2}}^{2} d t
\end{gathered}
$$

here we used the Hölder's inequality and

$$
\begin{gathered}
\int_{0}^{\infty}\left|\left(1+y^{2}\right) U_{1}(y) \frac{\sin (y t)}{y}\right|^{2} d y=\int_{0}^{\infty}\left|\left(1+y^{2}\right) U_{1}(y) \frac{\sin (y t)}{y t} \cdot t\right|^{2} d y \\
\lesssim \int_{0}^{\infty}\left|\left(1+y^{2}\right) U_{1}(y)\right|^{2} d y=\left\|u_{1}\right\|_{G_{\mu}^{1,2}}^{2}
\end{gathered}
$$

Consequently, we have

$$
\|u(t, \cdot)\|_{G_{\mu}^{1,2}}^{2} \lesssim \int_{0}^{T}\|f(t, \cdot)\|_{G_{\mu}^{1,2}}^{2} d t+\left\|u_{1}\right\|_{G_{\mu}^{1,2}}^{2}+\left\|u_{0}\right\|_{G_{\mu}^{1,2}}^{2}
$$

From (14), we obtain

$$
\|u\|_{C\left([0, T], G_{\mu}^{1,2}(I)\right)}^{2}:=\max _{0<t<T}\|u(t, \cdot)\|_{G_{\mu}^{1,2}}^{2} \lesssim\|f\|_{C\left([0, T], G_{\mu}^{1,2}(I)\right)}^{2}+\left\|u_{1}\right\|_{G_{\mu}^{1,2}}^{2}+\left\|u_{0}\right\|_{G_{\mu}^{1,2}}^{2} .
$$

Now, for $u_{t t}$ we have

$$
\begin{gathered}
\left\|u_{t t}(t, \cdot)\right\|_{2}^{2}=\left\|U_{t t}(t, \cdot)\right\|_{2}^{2}=\int_{0}^{\infty}\left|U_{t t}(t, y)\right|^{2} d y=\int_{0}^{\infty}\left|F(t, y)-y^{2} U(t, y)\right|^{2} d y \\
\lesssim \int_{0}^{\infty}|F(t, y)|^{2} d y+\int_{0}^{\infty}\left|y^{2} U(t, y)\right|^{2} d y \lesssim\|F(t, \cdot)\|_{2}^{2}+\int_{0}^{\infty}\left|\left(1+y^{2}\right) U(t, y)\right|^{2} d y \\
=\|f(t, \cdot)\|_{2}^{2}+\|u(t, \cdot)\|_{G_{\mu}^{1,2}}^{2},
\end{gathered}
$$

by using the Parseval formula (6). Thus, we obtain

$$
\begin{gathered}
\|u\|_{C^{2}\left([0, T], L^{2}(I)\right)}^{2} \lesssim\|f\|_{C\left([0, T], L^{2}(I)\right)}^{2}+\|u\|_{C\left([0, T], G_{\mu}^{1,2}(I)\right)}^{2} \\
\lesssim\|f\|_{C\left([0, T], G_{\mu}^{1,2}(I)\right)}^{2}+\|u\|_{C\left([0, T], G_{\mu}^{1,2}(I)\right)}^{2} \lesssim\|f\|_{C\left([0, T], G_{\mu}^{1,2}(I)\right)}^{2}+\left\|u_{1}\right\|_{G_{\mu}^{1,2}}^{2}+\left\|u_{0}\right\|_{G_{\mu}^{1,2}}^{2} .
\end{gathered}
$$

The existence is proved.

Let us suppose that $u_{1}$ and $u_{2}$ are two solutions of Problem 1 . Then $u(t, x)=u_{1}(t, x)-u_{2}(t, x)$ is the solution of following problem:

$$
u_{t t}(t, x)-S_{\mu} u(t, x)=0, u(0, x)=0, u_{t}(0, x)=0 .
$$

Above problem has only trivial solution $u(t, x) \equiv 0$, showing the uniqueness of the solution of the Problem 1 . The uniqueness is proved. 


\title{
Acknowledgements
}

This research was funded by the Science Committee of the Ministry of Education and Science of the Republic of Kazakhstan (Grant No. AP08052028).

\section{References}

1 Assal, M. (2008). Generalized wave equations in the setting of Bessel-Kingman hypergroups. An International Journal for Theory and Applications, 11(3), 249-257.

2 Zemanian, A.H. (1968). Generalized Integral Transformations. Interscience, New York.

3 Pathak, R.S. (1985). On Hankel transformable spaces and a Cauchy problem. Can. J. Math., 37(1), 84-106.

4 Albeverio, S., Hryniv, R., \& Mykytyuk, Ya. (2007). Invers spectral problems for Bessel operators. J. differential Equations, 241, 130-159.

5 Ciaurri, Ó., \& Roncal L. (2014). The wave equation for the Bessel Laplacian. J. Math. Anal. Appl., 409, 263-274.

6 Dostanić, M.R. (2014). Spectral properties of the operator of Bessel potential type. J. Math. Anal. Appl., 419, 255-272.

7 Haimo, D.T. (1965). Integral equations associated with Hankel convolutions. Transactions of the American Mathematical Society, 116, 330-375.

8 Hirschman, I.I. (1960). Variation diminishing Hankel transforms. J. Analyse Math., 8, 307-336.

9 Masood, K., Messaoudi, S., \& Zaman, F.D. (2002). Initial inverse problem in heat equation with Bessel operator. International Journal of Heat and Mass Transfer, 45, 2959-2965.

10 Zaidman, S. (1991). Distributions and Pseudo-differential Operators. Copublished in the United States with John Wiley and Sons, Inc., New York.

11 Watson, G.N. (1922). A treatise on the theory of Bessel functions. Cambridge at the university press.

12 Pathak, R.S., \& Pandey, P.K. (1997). Sobolev type spaces associated with Bessel Operators. Journal of mathematical analysis and applications, 215, 95-111.

\section{Б. Бекболат, Н. Тоқмағамбетов}

\section{Бессель операторы арқылы туындаған толқын теңдеуі үшін тұрақтылық нәтижелері}

\begin{abstract}
Мақалада Бессель операторы арқылы туындаған біртекті емес толқын теңдеуі қарастырылған. Бессель операторы арқылы туындаған біртекті емес толқын теңдеуі шешімінің бар және жалғыздығы дәлелденген. Шешімнің түрі келтірілген. Соболев типтес кеңістікте априорлы бағалаулар алынған. Бұл есеп алғаш рет M. Assal [1] жұмысында Bessel-Kingman гипергруппасында қарастырылған. [1] жұмысында қолданылған әдіс үйірткі теоремасына және оған байланысты бағалауларға негізделген. Мақала авторлары бұл жұмыста басқа әдіс қолданған. Есеп Соболев кеңістігі көзқарасынан зерттелген. Атап айтқанда, Ханкель түрлендіруі және Парсеваль формуласы, Ханкель түрлендіруі және Бессель дифференциалдық операторы арасында коммутация формуласы бар екені ескеріле отырып [2], кеңінен қолданылады.
\end{abstract}

Kiлm сөздер: Бессель операторы, толқын теңдеуі, Ханкель түрлендіруі, кері Ханкель түрлендіруі, Соболев типтес кеңістік. 
Б. Бекболат, Н. Токмагамбетов

\section{Результаты корректности волнового уравнения, порожденного оператором Бесселя}

В статье рассмотрено неоднородное волновое уравнение, порожденное оператором Бесселя. Доказаны существование и единственность решения неоднородного волнового уравнения, порожденного оператором Бесселя. Приведено представление решения. Получены априорные оценки в пространстве соболевского типа. Эта проблема впервые была рассмотрена в работе M. Assal [1] в гипергруппе Bessel-Kingman. Метод, используемый в [1], основан на теореме свертки и связанных с ней оценках. Авторами статьи использован другой подход. Проблема изучена с точки зрения пространств Соболева. А именно преобразование Ханкеля и формула Парсеваля широко применяются с учетом того, что между преобразованием Ханкеля и дифференциальным оператором Бесселя существует коммутационная формула [2].

Ключевые слова: оператор Бесселя, волновое уравнение, преобразование Ханкеля, обратное преобразование Ханкеля, пространство соболевского типа. 


\author{
N.A. Bokayev, A.A. Khairkulova* \\ L.N. Gumilyov Eurasian national university, Nur-Sultan, Kazakhstan \\ (E-mail: bokayev2011@yandex.ru, aitbekovna3@mail.ru)
}

\title{
On the boundedness of the fractional maximal operator on global Orlicz-Morrey spaces
}

\begin{abstract}
The article deals with the global Orlia-Morrey spaces $G M_{\Phi, \varphi, \theta}\left(R^{n}\right)$. We find sufficient conditions on pairs of functions $(\varphi, \eta)$ and $(\Phi, \Psi)$, which ensure the boundedness of the fractional maximal operator $M_{\alpha}$ from $G M_{\Phi, \varphi, \theta}\left(R^{n}\right)$ in $G M_{\Psi, \eta, \theta}\left(R^{n}\right)$. It is proved that under some additional conditions on the function $\varphi$, the conditions obtained are also necessary. In the proof, the boundedness condition is essentially used, the maximal Hardy-Littlewood functions and the estimate of the norm of the characteristic function in global Orlicz-Morrey spaces are used.
\end{abstract}

Keywords: Orlicz space, Morrey type space, the fractional maximal functions, the global Orlicz-Morrey spaces.

\section{Introduction}

The classical Morrey space was introduced in the works of Charles Morrey in 1938 [1] in connection with the study of the solution of quasilinear elliptic differential equations. Inrecent decades, the boundedness of various operators in spaces of Morrey type has been actively studied. This paper, we consider the boundedness of the fractional maximal functions in global Orlicz-Morrey spaces. We note that the issues of the boundedness of the fractional maximal operator and the Riesz potential in various function spaces are well studied. For classical Lebesgue spaces they are detailed in monographs $[2,3]$.

We give definitions of the classical operators of the theory of functions and various Morrey spaces of interest to us and some papers in which the boundedness of these operators in these spaces is considered.

The Hardy-Littlewood maximal operator

$$
M f(x)=\sup _{r>0} \frac{1}{|B(x, r)|} \int_{B(x, r)}|f(y)| d y
$$

is bounded on $L_{p}$ for $1<p<\infty$.

Let $f \in L_{1}^{\text {loc }}\left(R^{n}\right)$. The fractional maximal operator $M_{\alpha}$ are defined by

$$
M_{\alpha} f(x)=\sup _{r>0}|B(x, r)|^{\frac{\alpha}{n}-1} \int_{B(x, r)}|f(y)| d y, 0 \leq \alpha<n,
$$

where $B(x, r)$ is an open ball centered at a point $x \in R^{n}$ of radius $r>0$. If $\alpha=0$, then $M_{0} \equiv M$ is the Hardy-Littlewood maximal operator.

The classical Morrey spaces $M_{p, \lambda}\left(R^{n}\right)$ are defined as the set of all functions $f \in L_{p}^{\text {loc }}\left(R^{n}\right)$ for which

$$
\|f\|_{M_{p, \lambda}\left(R^{n}\right)}=\sup _{x, r>0} r^{-\lambda / p}\|f\|_{L_{p}(B(x, r))}
$$

where $0 \leq \lambda \leq n, 1 \leq p<\infty$. It's clear that $\|f\|_{M_{p, 0}\left(R^{n}\right)} \equiv\|f\|_{L_{p}\left(R^{n}\right)},\|f\|_{M_{p, \frac{n}{p}}\left(R^{n}\right)} \equiv\|f\|_{L_{\infty}\left(R^{n}\right)}$.

Let $1 \leq p \leq \infty, \omega$ be measurable non-negative function on $(0, \infty)$, not equivalent to zero. The generalized Morrey spaces $M_{p, \omega(\cdot)} \equiv M_{p, \omega(\cdot)}\left(R^{n}\right)$ are defined as the set of all functions $f \in L_{p}^{l o c}\left(R^{n}\right)$ with finite norm

$$
\|f\|_{M_{p, \omega(\cdot)}} \equiv \sup _{x \in R^{n}, r>0}\left(\omega(r)\|f\|_{L_{p}(B(x, r))}\right)<\infty .
$$

\footnotetext{
${ }^{*}$ Corresponding author.

E-mail: aitbekovna3@mail.ru
} 
The generalized Morrey space $M_{p, \omega(\cdot)}$ coincides with the classical Morrey spaces for $\omega(r)=r^{-\lambda}$ where $0 \leq \lambda \leq \frac{n}{p}$

The generalized Morrey space $M_{p, \omega(\cdot)} \equiv M_{p, \omega(\cdot)}\left(R^{n}\right)$ introduced by (Mizuhara, Nakai 1990, 1994) $[4,5]$ and they were reviewed in [6], in which various sufficient conditions are given for the boundedness of the maximal Hardy-Littlewood functions, the fractional maximal functions and the Riesz potential in these spaces. Global Morrey-type spaces $G M_{p, \theta, \omega(\cdot)}\left(R^{n}\right)$ and the local Morrey spaces were considered by of Burenkov V.I., Guliev V.S., A.Gogatishvili, R. Mustafaev ([7-9]), in which various sufficient, and for some values of the parameters, necessary conditions for the boundedness of the maximal Hardy-Littlewood functions, the fractional maximal functions in these spaces are obtained.

Let $1 \leq p \leq \infty, 1 \leq \theta \leq \infty, \omega$ be measurable non-negative function on $(0, \infty)$, not equivalent to zero. The global Morrey spaces $G M_{p, \theta, \omega(\cdot)} \equiv G M_{p, \theta, \omega(\cdot)}\left(R^{n}\right)$ are defined as the set of all functions $f \in L_{p}^{\text {loc }}\left(R^{n}\right)$ with finite quasinorm

$$
\|f\|_{G M_{p, \theta, \omega(\cdot)}} \equiv \sup _{x \in R^{n}}\|\omega(r)\| f\left\|_{L_{p}(B(x, r))}\right\|_{L_{\theta}(0, \infty)},
$$

where $B(x, r)$ is an open ball centered at a point $x \in R^{n}$ of radius $r>0$.

The spaces $G M_{p, \theta, \omega(\cdot)}\left(R^{n}\right)$ coincides with the generalized Morrey space $M_{p, \omega(\cdot)}\left(R^{n}\right)$ at $\theta=\infty$.

The results on the boundedness of various classical operators in the theory of functions in global and local Morrey spaces (until 2013) are presented in review articles by V. I. Burenkov [10, 11].

Another well-known space that generalizes Lebesgue space $L_{p}$ is the space introduced by Orlicz ([12]).

We recall the definition of Young functions.

A function $\Phi:[0,+\infty] \rightarrow[0, \infty]$ is called Young's function if $\Phi$ is a convex function, left continuous, and such that

$$
\lim _{r \rightarrow+0} \Phi(r)=\Phi(0)=0, \lim _{r \rightarrow+\infty} \Phi(r)=\infty .
$$

From the convexity of the function and $\Phi(0)=0$ it follows that any Young function is increasing.

If there is $s \in(0, \infty)$ such that $\Phi(s)=\infty$, then $\Phi(r)=\infty$ for $r \geq s$.

Let $E$ be the set of all Young functions $\Phi$ such that

$$
0<\Phi(r)<+\infty
$$

for

$$
0<r<+\infty
$$

If $\Phi \in E$, then $\Phi$ is absolutely continuous on every closed interval in $[0, \infty)$ and bijective from $[0, \infty)$ to itself.

For a Young function $\Phi$, the set

$$
L_{\Phi}\left(R^{n}\right)=\left\{f \in L_{1}^{\text {loc }}\left(R^{n}\right): \int_{R^{n}} \Phi(k|f(x)|) d x<+\infty, \text { for some } k>0\right\}
$$

is called Orlicz space. In the works $[13,14]$ the questions of the boundedness of classical operators of the theory of functions in Orlicz spaces were studied.

If $\Phi(r)=r^{p}, 1 \leq p<\infty$, then $L_{\Phi}\left(R^{n}\right)=L_{p}\left(R^{n}\right)$. If $\Phi(r)=0(0 \leq r \leq 1)$ and $\Phi(r)=\infty(r>1)$, then $L_{\Phi}\left(R^{n}\right)=L_{\infty}\left(R^{n}\right)$.

$L_{\Phi}\left(R^{n}\right)$ is a Banach space with respect to the norm

$$
\|f\|_{L_{\Phi}\left(R^{n}\right)}=\inf \left\{\lambda>0: \int_{R^{n}} \Phi\left(\frac{|f(x)|}{\lambda}\right) d x \leq 1\right\} .
$$

We note that

$$
\int_{R^{n}} \Phi\left(\frac{|f(x)|}{\|f\|_{L_{\Phi}}}\right) d x \leq 1 .
$$

For Young's function $\Phi$ and $0 \leq s \leq+\infty$ let

$$
\Phi^{-1}(s)=\inf \{r \geq 0: \Phi(r)>s\} .
$$

If $\Phi \in E$, then $\Phi^{-1}$ this is the usual inverse function for $\Phi$. We note that

$$
\Phi\left(\Phi^{-1}(r)\right) \leq r \leq \Phi^{-1}(r), \text { for } 0 \leq r<\infty .
$$


A Young function $\Phi$ is said to satisfy the $\triangle_{2}$-condition, denoted by $\Phi \in \triangle_{2}$, if

$$
\Phi(2 r) \leq k \Phi(r), \text { for } r>0
$$

for some $k>1$.

A Young function $\Phi$ is said to satisfy the $\nabla_{2}$-condition, denoted also by $\Phi \in \nabla_{2}$, if

$$
\Phi(r) \leq \frac{1}{2 k} \Phi(k r), r \geq 0
$$

for some $k>1$. The function $\Phi(r)=r$ satisfies the $\triangle_{2}$-condition but does not satisfy the $\nabla_{2}$-condition. If $1<p<\infty$, then $\Phi(r)=r^{p}$ satisfies the $\nabla_{2}$-condition but does not satisfy the $\triangle_{2}$-condition.

A Young function $\Phi$ is said to satisfy the $\triangle^{\prime}$ - condition, denoted also as $\Phi \in \triangle^{\prime}$, if

$$
\Phi(t r) \leq k \Phi(t) \Phi(r), t, r \geq 0
$$

for some $k>1$. If $\Phi \in \nabla_{2}$, then $\Phi \in E$.

The boundedness conditions for classical operators of the theory of functions in generalized Orlicz-Morrey spaces $M_{\Phi, \varphi}$ were considered by V.S.Guliyev, F.Deringoz, S.Samko and others [15-18].

The purpose of this paper is to consider global Orlicz-Morrey spaces $G M_{\Phi, \varphi, \theta}\left(R^{n}\right)$ (the corresponding definition is given in the next section) and find conditions for the boundedness the fractional maximal functions in these spaces.

\section{Definitions, notation, and auxiliary statements}

We give the definition of global Orlicz-Morrey spaces in the following way:

Definition 1. Let $\varphi(x, r)$ be a positive, measurable function on $R^{n} \times(0, \infty)$ and $\Phi$ be a Young function, $1 \leq \theta \leq \infty$. We defined the global Orlicz-Morrey spaces $G M_{\Phi, \varphi, \theta}=G M_{\Phi, \varphi, \theta}\left(R^{n}\right)$ as the set of all functions $f \in L_{\Phi}^{\text {loc }}\left(R^{n}\right)$ with finite quasinorm

$$
\|f\|_{G M_{\Phi, \varphi, \theta}}=\sup _{x \in R^{n}}\left\|\varphi(x, r)^{-1} \Phi^{-1}\left(|B(x, r)|^{-1}\right)\right\| f\left\|_{L_{\Phi}(B(x, r))}\right\|_{L_{\theta}(0, \infty)} .
$$

At $\theta=\infty$ the corresponding space is called the generalized Orlicz-Morrey space $M_{\Phi, \varphi}$.

Let $\varphi(x, r)$ be a positive, measurable function on $R^{n} \times(0, \infty)$ and $\Phi$ be a Young function. We denote by $M_{\Phi, \varphi}$ the generalized Orlicz-Morrey spaces, the space of all functions $f \in L_{\Phi}^{\text {loc }}\left(R^{n}\right)$ with finite quasinorm

$$
\|f\|_{M_{\Phi, \varphi}}=\sup _{x \in R^{n}, r>0} \varphi(x, r)^{-1} \Phi^{-1}\left(|B(x, r)|^{-1}\right)\|f\|_{L_{\Phi}(B(x, r))} .
$$

At $\Phi(r)=r^{p}, 1 \leq p<\infty$ the corresponding global Orlicz-Morrey space is denoted by $G M_{p, \varphi, \theta}\left(R^{n}\right)$ :

$$
G M_{p, \varphi, \theta}\left(R^{n}\right)=\left.G M_{\Phi, \varphi, \theta}\left(R^{n}\right)\right|_{\Phi(r)=r^{p}} .
$$

At

$$
\varphi(x, r)=\left(\Phi^{-1}\left(r^{-n}\right) / \Phi^{-1}\left(r^{-\lambda}\right)\right)
$$

the corresponding global Orlicz-Morrey space is denoted by $G M_{\Phi, \lambda, \theta}\left(R^{n}\right)$ :

$$
G M_{\Phi, \lambda, \theta}\left(R^{n}\right)=\left.G M_{\Phi, \varphi}\right|_{\varphi(x, r)=\Phi^{-1}\left(r^{-n}\right) / \Phi^{-1}\left(r^{-\lambda}\right)}
$$

At $\Phi(r)=r^{p}, 1 \leq p<\infty$ and $\varphi(x, r)=\left(\Phi^{-1}\left(r^{-n}\right) / \Phi^{-1}\left(r^{-\lambda}\right)\right)$ the global Orlicz-Morrey space coincides with the Morrey space, i.e. $G M_{p, \lambda, \infty}\left(R^{n}\right)=M_{p, \lambda}\left(R^{n}\right)$.

Let $\Phi$ be a Young function. We denote by $\Omega_{\Phi}$ the sets of all positive measurable functions $\varphi$ on $R^{n} \times(0, \infty)$ such that for all $t>0$,

$$
\sup _{x \in R^{n}}\left\|\frac{\Phi^{-1}\left(|B(x, r)|^{-1}\right)}{\varphi(x, r)}\right\|_{L_{\infty}(t, \infty)}<\infty
$$

and

$$
\sup _{x \in R^{n}}\left\|\varphi(x, r)^{-1}\right\|_{L_{\infty}(0, t)}<\infty
$$

respectively. 
We give auxiliary statements that we will need in the proof of the main statement.

By $\chi_{B}$ we denote the characteristic function of the set $B$.

Lemma 1. [16] Let $\Phi$ be a Young's function and $B$ a set in $R^{n}$ finite Lebesgue measure. Then

$$
\left\|\chi_{B}\right\|_{L_{\Phi}}=\frac{1}{\Phi^{-1}\left(|B|^{-1}\right)} .
$$

Lemma 2. [16] Let $0<\alpha<n, 1<\theta<\infty$ and function $\varphi(x, t)$ satisfies condition

$$
t^{\alpha} \varphi(x, t)+\left\|r^{\alpha-1}\right\|_{L_{\theta}(t, \infty)} \leq C \varphi(x, t)^{\beta}
$$

for some $\beta \in(0,1)$ and for each $x \in R^{n}$ and $t>0$. Then there is pointwise inequality

$$
M_{\alpha} f(x) \leq C(M f(x))^{\beta} \cdot\|f\|_{G M_{\Phi, \varphi, \theta}} .
$$

Lemma 3. [16] If $B_{0}=B\left(x_{0}, r_{0}\right)$, then $r_{0}^{\alpha} \leq C M_{\alpha} \chi_{B_{0}}(x)$ for every $x \in B_{0}$.

A function $\varphi:(0, \infty) \rightarrow(0, \infty)$ is said to be almost increasing (respectively, almost decreasing) if there exists a constant $c>0$ such that

$$
\varphi(r) \leq C \varphi(s)(\text { respectively } \varphi(r) \geq C \varphi(s), \text { for } r \leq s) .
$$

For Young's function $\Phi$ we denote by $£_{\Phi}$ the set of all almost decreasing functions $\varphi:(0, \infty) \rightarrow(0, \infty)$ such that $t \in(0, \infty) \mapsto \frac{\varphi(t)}{\Phi^{-1}\left(t^{-n}\right)}$ is almost increasing.

Lemma 4. Let $B_{0}:=B\left(x_{0}, r_{0}\right)$. If $\varphi \in £_{\Phi}$ then there exist $c>0$ such that

$$
\frac{1}{\varphi\left(r_{0}\right)} \leq\left\|\chi_{B_{0}}\right\|_{G M_{\Phi, \varphi, \theta}} \leq \frac{C}{\varphi\left(r_{0}\right)} .
$$
have:

Proof. Let $B_{s}=B(x, s)$ arbitrary ball from $R^{n}$. If $s \leq r$, then $\varphi(r) \leq C \varphi(s)$ and according to Lemma 1 we

$$
\varphi(s)^{-1} \Phi^{-1}\left(|B|^{-1}\right)\left\|\chi_{B}\right\|_{L_{\Phi}(B)} \leq \frac{1}{\varphi(s)} \leq \frac{C}{\varphi(r)} .
$$

Hence

$$
\left\|\varphi(s)^{-1} \Phi^{-1}\left(|B|^{-1}\right)\right\| \chi_{B}\left\|_{L_{\Phi}(B)}\right\|_{L_{\theta}(0, \infty)} \leq \frac{C}{\|\varphi(r)\|_{L_{\theta}(0, \infty)}} .
$$

Means

$$
\left\|\chi_{\Phi}\right\|_{G M_{\Phi, \varphi, \theta}} \leq \frac{C}{\|\varphi(r)\|_{L_{\theta}(0, \infty)}} .
$$

If $s \geq r$ then by $\varphi \in £_{\Phi}$ we have:

$$
\frac{\varphi(r)}{\Phi^{-1}\left(\left|B_{0}\right|^{-1}\right)} \leq C \frac{\varphi(s)}{\Phi^{-1}\left(|B|^{-1}\right)}
$$

therefore

it follows that

$$
\varphi(r)^{-1} \Phi^{-1}\left(|B|^{-1}\right)\left\|\chi_{B_{0}}\right\|_{L_{\Phi}(B)} \leq \frac{C}{\varphi(r)},
$$

$$
\left\|\chi_{B}\right\|_{G M_{\Phi, \varphi, \theta}} \leq \frac{C}{\|\varphi(r)\|_{L_{\theta}(0, \infty)}} .
$$

Lemma 4 is proved.

\section{Results for fractional maximal operator in global spaces of Orlicz-Morrey type}

Theorem 1. Let $\Phi \in \triangle^{\prime} \cap \nabla_{2}$, and $0<\alpha<n, 1<\theta<\infty$. Let $\varphi \in \Omega_{\Phi}$ satisfies condition

$$
r^{\alpha} \varphi(x, r)+\sup _{r<t<\infty} t^{\alpha} \varphi(x, t) \leq C \varphi(x, r)^{\beta}
$$


for some $\beta \in(0,1)$ and for every $x \in R^{n}$ and $r>0$. Define $\eta(x, r)=\varphi(x, r)^{\beta}$, and $\Psi(r)=\Phi\left(r^{1 / \beta}\right)$. The maximal operator $M$ the bounded in $G M_{\Phi, \varphi, \theta}\left(R^{n}\right)$, then the operator $M_{\alpha}$ is bounded from $G M_{\Phi, \varphi, \theta}\left(R^{n}\right)$ to $G M_{\Psi, \eta, \theta}\left(R^{n}\right)$.

Proof. By Lemma 2, we have

$$
M_{\alpha} f(x) \leq C\left(M_{\alpha} f(x)\right)^{\beta} \cdot\|f\|_{G M_{\Phi, \varphi, \theta}}^{1-\beta}, x \in R^{n},
$$

therefore

$$
\left\|M_{\alpha} f(x)\right\|_{L_{\Psi(B)}} \leq C\left\|\left(M_{\alpha} f\right)^{\beta}\right\|_{L_{\Psi}(B)} \cdot\|f\|_{G M_{\Phi, \varphi, \theta}}^{1-\beta}, x \in R^{n}
$$

where $B=B(x, t)$.

It follows from the definition of Orlicz space that

$$
\int_{B} \Psi\left(\frac{(M f(x))^{\beta}}{\|M f\|_{L_{\Phi}(B)}}\right) d x=\int_{B}\left(\Phi \frac{M f(x))}{\|M f\|_{L_{\Phi}(B)}}\right) d x \leq 1 .
$$

Hence

SO

$$
\left\|(M f)^{\beta}\right\|_{L_{\Psi}(B)} \leq\|M f\|_{L_{\Phi}(B)}^{\beta}
$$

$$
\left\|M_{\alpha} f\right\|_{L_{\Psi(B)}} \leq C\|M f\|_{L_{\Psi}(B)}^{\beta} \cdot\|f\|_{G M_{\Phi, \varphi, \theta}}^{1-\beta} .
$$

Based on this inequality, given the boundedness of the maximal operator in $G M_{\Phi, \varphi, \theta}$, we obtain

$$
\begin{gathered}
\left\|M_{\alpha} f\right\|_{G M_{\Psi, \eta, \theta}}=\sup _{x \in R^{n}}\left\|\eta(x, t)^{-1} \Psi^{-1}\left(|B|^{-1}\right)\right\| M_{\alpha} f\left\|_{L_{\Psi}(B)}\right\|_{L_{\theta}(0, \infty)} \leq \\
\leq C\|f\|_{G M_{\Phi, \varphi, \theta}}^{1-\beta} \cdot \sup _{x \in R^{n}}\left\|\varphi(x, t)^{-1} \Phi^{-1}\left(|B|^{-1}\right)\right\| M f\left\|_{L_{\Phi}(B)}^{\beta}\right\|_{L_{\theta}(0, \infty)}= \\
=C\|f\|_{G M_{\Phi, \varphi, \theta}}^{1-\beta}\|M f\|_{G M_{\Phi, \varphi, \theta}}^{\beta} \leq C \cdot\|f\|_{G M_{\Phi, \varphi, \theta}} .
\end{gathered}
$$

Theorem 1 is proved.

Theorem 2. Let $\Phi \in \triangle^{\prime}, 0<\alpha<n, 1<\theta<\infty, \varphi \in \Omega_{\Phi}, \beta \in(0,1), \eta(t) \equiv \varphi(t)^{\beta}$, and $\Psi(t) \equiv \Phi\left(t^{1 / \beta}\right)$.

1. Let $\Phi \in \nabla_{2}$ and the maximal operator $M$ the bounded in $G M_{\Phi, \varphi, \theta}\left(R^{n}\right)$, then the condition

$$
t^{\alpha} \varphi(t)+\left\|r^{\alpha-1} \varphi(r)\right\|_{L_{\theta^{\prime}}(t, \infty)} \leq C \varphi(t)^{\beta}
$$

for all $t>0$, where $C>0$ does not depend on $\mathrm{t}$, is sufficient for the boundedness of $M_{\alpha}$ from $G M_{\Phi, \varphi, \theta}\left(R^{n}\right)$ to $G M_{\Psi, \eta, \theta}\left(R^{n}\right)$.

2. If $\varphi \in £_{\Phi}$, then the condition

$$
\left\|\frac{t^{\alpha}}{\varphi(t)^{\beta}}\right\|_{L_{\theta}(0, \infty)} \leq \frac{C}{\|\varphi(t)\|_{L_{\theta}(0, \infty)}}
$$

is necessary for boundedness of the operator $M_{\alpha}$ from $G M_{\Phi, \varphi, \theta}\left(R^{n}\right)$ to $G M_{\Psi, \eta, \theta}\left(R^{n}\right)$.

3. Let $\Phi \in \nabla_{2}$. If $\varphi \in £_{\Phi}$ satisfies the regularity condition

$$
\left\|r^{\alpha-1} \varphi(r)\right\|_{L_{\theta^{\prime}}(t, \infty)} \leq C t^{\alpha} \varphi(t)
$$

for all $t>0$, where $C>0$ independent of $t$, then the condition (1) is necessary and sufficient for the boundedness $M_{\alpha}$ from $G M_{\Phi, \varphi, \theta}\left(R^{n}\right)$ to $G M_{\Psi, \eta, \theta}\left(R^{n}\right)$.

Proof. The first part follows from Theorem 1 . To prove the second part, we put $B_{0}=B\left(x_{0}, t\right)$, by Lemma 3 , we have

$$
t^{\alpha} \leq C M_{\alpha} \chi_{B_{0}}(x), x \in B_{0} .
$$

We estimate by Lemma 1 ana Lemma 3

$$
t^{\alpha} \leq C \Psi^{-1}\left(\left|B_{0}\right|^{-1}\right)\left\|M_{\alpha} \chi_{B_{0}}\right\|_{L_{\Psi}\left(B_{0}\right)} .
$$

Hence

$$
\frac{t^{\alpha}}{\eta(t)} \leq C \eta^{-1}(t) \Psi^{-1}\left(\left|B_{0}\right|^{-1}\right)\left\|M_{\alpha} \chi_{B_{0}}\right\|_{L_{\Psi}\left(B_{0}\right)}
$$

Mathematics series. № 1(101)/2021 
Therefore

$$
\left\|\frac{t^{\alpha}}{\eta(t)}\right\|_{L_{\theta}(0, \infty)} \leq C\left\|M_{\alpha} \chi_{B_{0}}\right\|_{G M_{\Psi, \eta, \theta}} \leq C\left\|\chi_{B_{0}}\right\|_{G M_{\Phi, \varphi, \theta}} \leq C \frac{1}{\|\varphi(r)\|_{L_{\theta}(r, \infty)}} .
$$

The third part follows from parts 1 and 2. Theorem 2 is proved.

Corollary 1. Let $0<\alpha<n, 1<\theta<\infty, 1<p<q<\infty$ and $\varphi \in \Omega_{p} \equiv \Omega_{t^{p}}$.

1. If $\varphi(t)$ satisfics

$$
\sup _{r<t<\infty} \frac{e s s \inf _{t<s<\infty} \varphi(s) s^{\frac{n}{p}}}{t^{\frac{n}{p}}} \leq C \varphi(r)
$$

then the condition

$$
t^{\alpha} \varphi(t)+\sup _{t<r<\infty} r^{\alpha} \varphi(r) \leq C \varphi(t)^{\frac{p}{q}}
$$

for all $t>0$, where $C>0$ independent of $t$, is sufficient for the boundedness of $M_{\alpha}$ from $G M_{p, \varphi, \theta}\left(R^{n}\right)$ to $G M_{q, \varphi^{\frac{p}{q}}, \theta}\left(R^{n}\right)$.

2. If $\varphi \in £_{p} \equiv £_{t^{p}}$, then the condition

$$
t^{\alpha} \varphi(t) \leq C \varphi(t)^{\frac{p}{q}}
$$

for all $t>0$, where $C>0$ independent of $t$, is necessary for the boundedness of $M_{\alpha}$ from $G M_{p, \varphi, \theta}\left(R^{n}\right)$ to $G M_{q, \varphi^{\frac{p}{q}, \theta}}\left(R^{n}\right)$.

3. If $\varphi \in £_{p}$ then the condition (2) is necessary and sufficient for the boundedness of $M_{\alpha}$ from $G M_{p, \varphi, \theta}\left(R^{n}\right)$ to $G M_{q, \varphi^{\frac{p}{q}, \theta}}\left(R^{n}\right)$.

If we take $\varphi(t)=\frac{\Phi^{-1}\left(t^{-n}\right)}{\Phi^{-1}\left(t^{-\lambda}\right)}, 0 \leq \lambda \leq n, \Psi(t) \equiv \Phi\left(t^{\frac{1}{\beta}}\right), \beta \in(0,1)$,

$$
\eta(t) \equiv \varphi(t)^{\beta}=\left(\frac{\Phi^{-1}\left(t^{-n}\right)}{\Phi^{-1}\left(t^{-\lambda}\right)}\right)^{\beta}=\frac{\Psi^{-1}\left(t^{-n}\right)}{\Psi^{-1}\left(t^{-\lambda}\right)}
$$

at Theorem 2 we get the following result.

Corollary 2. Let $\Phi \in \triangle^{\prime} \cap \nabla_{2}, \Psi(t) \equiv \Phi\left(t^{1 / \beta}\right)$ and $\beta \in(0,1)$. If

$$
\sup _{t<r<\infty} r^{\alpha} \frac{\Phi^{-1}\left(r^{-n}\right)}{\Phi^{-1}\left(r^{-\lambda}\right)} \leq C t^{\alpha} \frac{\Phi^{-1}\left(t^{-n}\right)}{\Phi^{-1}\left(t^{-\lambda}\right)}
$$

for all $t>0$, where $C>0$ independent of $t$, then the condition

$$
t^{\alpha} \leq C\left[\frac{\Phi^{-1}\left(t^{-n}\right)}{\Phi^{-1}\left(t^{-\lambda}\right)}\right]^{\beta-1}
$$

for all $t>0$, where $C>0$ independent of $t$, is necessary and sufficient for the boundedness of $M_{\alpha}$ from $G M_{\Phi, \lambda}\left(R^{n}\right)$ to $G M_{\Psi, \lambda}\left(R^{n}\right)$.

Remark. The Theorem 1 and 2 are an Adams type result. The similar theorem for the generalized OrliczMorrey spaces $M_{\Phi, \varphi}$ was proved in [16-18].

\section{References}

1 Morrey, Ch. (1938). On the solutions of quasi-linear elliptic partial differential equations. Trans. Amer. Math. Soc., 126-166.

2 Stein, E.M. (1970) Singular Integrals and Differentiability Properties of Functions. Princeton, New Jersey: Princetion University Press.

3 Torchinsky, A. (1986) Real-Variable Methods in Harmonic Analysis. Academic Press INC.

4 Mizuhara, T. (1991). Boundedness of some classical operators on generalized Morrey spaces Harmonic Analisis (S.Igari, Editor) ICM 90 Satellite Proceedings, Springer-Verlag, Tokyo, 183-189.

5 Nakai, E. (1994). Hardy-Littlewood maximal operator, singular integral operators and the Riesz potential on generalized Morrey spaces. Math. Nachr., 166(1), 95-103. 
6 Guliyev, V.S. (2009). Boundedness of the maximal, potential and singular operators in the generalized Morrey spaces. J.Inequal. Appl., 503948.

7 Burenkov, V.I., \& Guliyev, H.V. (2004). Necessary and sufficient conditions for boundedness of the maximal operator in the local Morrey-type spaces. Studia Mathematica., 163(2), 157-176.

8 Burenkov, V.I., Gogatishvili, A., Guliyev, V.S., \& Mustafaev, R. (2010). Boundedness of the maximal operator in the local Morrey-type spaces. Complex Analysis and Elliptic Equations 55(8-10), 739-758.

9 Burenkov V.I., \& Goldman, M.L. (2014). Necessary and sufficient conditions for boundedness of the maximal operator from Lebesgue spaces to Morrey-type spaces. Mathematical Inequalities and Applications. Accepted for publication. Math.Inequal.Appl. 17(2), 401-418.

10 Burenkov, V.I. (2012). Recent progress in studying the boundedness of classical operators of real analysis in general Morrey-type spaces I. Eurasian Math. J. 3(3), 11-32.

11 Burenkov, V.I. (2013). Recent progress in studying the boundedness of classical operators of real analysis in general Morrey-type spaces II. Eurasian Math. J. 4(1), 21-45.

12 Krasnoselskii, M.A., \& Rutickii, Ja.B. (1961). Convex Functions and Orlicz Spaces. P.Noordhoff, Groningen, The Netherlands.

13 Kita, H. (1997). On Hardy-Littlewood maximal functions in Orlicz spaces. Math. Nachr., 183, $135-155$.

14 Cianchi, A. (1999). Strong and weak type inequalities for some classical operators in Orlicz spaces. J. London Math. Soc. 60(2), 187-202.

15 Deringoz, F., Guliyev, V.S., \& Samko, S. (2014). Boundedness of the maximal operator and singular operators on generalized Orlicz-Morrey spaces. Operator Theory, Operator Algebras and Applications, Series: Operator Theory: Advances and Applications, 242, 139-158.

16 Deringoz, F., Guliyev, V.S., \& Hasanov, S.G. (2017). A characterizations for Adams-type boundedness of the fractional maximal operator on generalized Orlicz-Morrey spaces. arXiv: 1610.09823v2 [math.FA], $1-20$.

17 Deringoz, F., \& Guliyev, V.S. (2014). On the Riesz potential and its commutators on generalized OrliczMorrey spaces. Hindawi Publishing Corporation Journal of Function Spaces Volume, 1-11.

18 Sawano, Y., Sugano, S., \& Tanaka, H. (2012). Orlicz-Morrey spaces and fractional operators. Potential Analysis, 3(4), 517-556.

\author{
Н.А. Бокаев, А.А. Хайркулова
}

\title{
Бөлшек максималды оператордың Орлич-Морри кеңістігіндегі шекарасы
}

\begin{abstract}
Мақалада $G M_{\Phi, \varphi, \theta}\left(R^{n}\right)$ ғаламдық Орлич-Морри кеңістігі қарастырылған. Авторлар $(\varphi, \eta)$ және $(\Phi, \Psi)$ функцияларының жұптары үшін $M_{\alpha}$ бөлшек максималды операторының шекарасын қамтамасыз ететін жеткілікті шарттарды тапқан. $G M_{\Phi, \varphi, \theta}\left(R^{n}\right)$ кеңістігінен $G M_{\Psi, \eta, \theta}\left(R^{n}\right)$ кеңістігіне $\varphi$ функциясы бойынша кейбір қосымша заңдылық шарттарында алынған шарттар да қажет екендігі дәлелденді. Дәлелдеуде негізінен шектілік шарты максималды Харди-Литтвуд функциялары және ғаламдық Орлич-Морри кеңістігіндегі сипаттамалық функция нормасы қолданылған.
\end{abstract}

Kiлm сөздер: Орлич кеңістігі, Морри типті кеңістік, бөлшек максималды функциялар, жалпыланған Орлич-Морри кеңістіктері. 
Н.А. Бокаев, А.А. Хайркулова

\section{Об ограниченности дробного максимального оператора в глобальных пространствах Орлича-Морри}

В статье рассмотрены глобальные пространства Орлича-Морри $G M_{\Phi, \varphi, \theta}\left(R^{n}\right)$. Авторы находят достаточные условия на пары функций $(\varphi, \eta)$ и $(\Phi, \Psi)$, которые обеспечивают ограниченность дробномаксимального оператора $M_{\alpha}$ из $G M_{\Phi, \varphi, \theta}\left(R^{n}\right)$ в пространство $G M_{\Psi, \eta, \theta}\left(R^{n}\right)$. Доказано, что при некоторых дополнительных условиях регулярность на функцию $\varphi$, полученные условия являются необходимыми. При доказательстве существенно использованы условие ограниченности, максимальные функции Харди-Литлвуда и оценка нормы характеристической функции в глобальных пространствах Орлича-Морри.

Ключевые слова: пространство Орлича, пространство типа Морри, дробные максимальные функции, глобальные пространства Орлича-Морри. 


\author{
S.M. Davoodi*, N.A. Abdul Rahman \\ School of Mathematical Sciences, Universiti Sains Malaysia, Penang, Malaysia \\ (E-mail: smd1376@gmail.com,aswad.rahman@usm.my)
}

\title{
Predicting the optimal solution in fuzzy linear programming problem
}

\begin{abstract}
In this paper we try to define a percentage form of LR fuzzy numbers which is a useful form of fuzzy numbers and its' arithmetics. So, we show how the maximum variation range of optimal value of fuzzy objective function can be predicted by using this form of fuzzy numbers. Since fuzzy problems are generally solved through a complicated manner, the purpose of this study is releasing a kind of prediction for the final solution in the way that the manager can access to an outlook to optimal solution $\left(Z^{*}\right)$ without solving the problem. Finally, optimal value of fuzzy objective function on fuzzy linear programming is predicted when maximum variation range of fuzzy variable have been predetermined.
\end{abstract}

Keywords: Percentage form of fuzzy numbers, Fuzzy number, Fuzzy linear programming, Fuzzy Arithmetic

\section{Introduction}

In the recent past the fields of management science, operational research and industrial engineering have dedicated a lot of attention to decision making theories, decision making methods and application. For the past sixty years, many research works in these areas have been documented. However, there is a need to develop new methods that would fit real world problems in the context of linear programming. The traditional methods of linear programming modeling require precise model parameters and this, is not obtainable in real world problems. So, researchers usually estimate model parameters by themselves and These estimated values of the model parameters may not be precise.

Tanaka et al. [1] proposed the concept of fuzzy mathematical programming based on the fuzzy decision framework of Bellman and Zadeh [2]. Zimmerman [3] introduced the first formulation of fuzzy linear programming (FLP) to address the impreciseness of the parameters in linear programming (LP) problems with fuzzy constraints and objective functions. A number of researchers have exhibited their interest to solve the FLP problems [4-13] and fully FLP problems [14-23].

A new form of fuzzy numbers called percentage form of LR fuzzy numbers (PLR fuzzy number) is now introduced in this paper. This form of fuzzy numbers are applied to many discipline such as industrial application, mathematical modeling and management sciences. Arithmetic operations of this form of fuzzy numbers and some properties of it is studied. Also the prediction process of fuzzy optimal solution and its variation range is described in this paper according to properties of this form of fuzzy numbers.

The solution of FLP problems and fully FLP problems cannot be calculated by applying the majority of existing methods in which some or all the parameters are represented by unrestricted LR fuzzy numbers. In addition, there are large computational procedure with some existing methods [20]. By predicting the range of fuzzy numbers' change and reaching to an outlook of optimal solution and also having an alternating fuzzy variables' frequency range on demand, we are able to find the optimal solution with ideal frequency range.

\section{Preliminary Concepts}

In this section, we introduce some basic definitions on fuzzy set theory and the main concepts needed in the paper.

Definition 1. Let $X$ be a collection of objects denoted generically by $x$. $\widetilde{A}$ is called $a$ fuzzy set in $X$ if $\widetilde{A}$ is a set of ordered pairs:

$$
\widetilde{A}=\left\{\left(x, \mu_{\widetilde{A}}(x)\right) \mid x \in X\right\}
$$

where $\mu_{\widetilde{A}}(x)$ is a membership function of $x$ in $\mu_{\widetilde{A}}$ such that $\mu_{\widetilde{A}}(x): X \rightarrow[0,1]$.

\footnotetext{
${ }^{*}$ Corresponding author.

E-mail:smd1376@gmail.com
} 
Definition 2. A fuzzy number $\widetilde{A}$ is said to be a $L R$-flat fuzzy number, $\widetilde{A}=\left(a^{l}, a^{u}, \alpha, \beta\right)_{L R}$, if its membership function is defined as

$$
\mu_{\widetilde{A}}(x)= \begin{cases}L\left(\frac{a^{l}-x}{\alpha}\right) & , \quad x<a^{l} \\ 1 & , \quad a^{l} \leq x \leq a^{u} \\ R\left(\frac{x-a^{u}}{\beta}\right) & , \quad x>a^{u} \\ 0 & , \text { otherwise }\end{cases}
$$

where $L$ and $R$ are reference functions, i.e., $L, R:[0,+\infty) \rightarrow[0,1]$ are non-increasing that $L(0)=R(0)=$ 1 and $L(1)=R(1)=0$. The membership function of a $L R$ fuzzy number, $\widetilde{A}=(a, \alpha, \beta)_{L R}$, can be also defined as

$$
\mu_{\widetilde{A}}(x)= \begin{cases}L\left(\frac{a-x}{\alpha}\right) & , \quad x \leq a \\ R\left(\frac{x-a}{\beta}\right), & x>a \\ 0 & , \text { otherwise }\end{cases}
$$

Definition 3. Let $\widetilde{A}_{1}=\left(a_{1}, \alpha_{1}, \beta_{1}\right)_{L R}$ and $\widetilde{A}_{2}=\left(a_{2}, \alpha_{2}, \beta_{2}\right)_{L R}$ be $L R$ fuzzy numbers and $r \in \mathbb{R},(r \neq 0)$ then arithmetic on fuzzy numbers are defined

$$
\begin{aligned}
& \widetilde{A}_{1} \oplus \widetilde{A}_{2}=\left(a_{1}+a_{2}, \alpha_{1}+\alpha_{2}, \beta_{1}+\beta_{2}\right)_{L R} \\
& r \widetilde{A}_{1}= \begin{cases}\left(r a_{1}, r \alpha_{1}, r \beta_{1}\right)_{L R} & , \quad r>0 \\
\left(r a_{1},-r \beta_{1},-r \alpha_{1}\right)_{L R} & , \quad r<0\end{cases} \\
& \widetilde{A}_{1} \otimes \widetilde{A}_{2}=\left\{\begin{array}{rlll}
\left(a_{1} a_{2}, a_{1} \alpha_{2}+a_{2} \alpha_{1}, a_{1} \beta_{2}+a_{2} \beta_{1}\right)_{L R} & , \text { where } \widetilde{A}_{1} \succ 0 & , \widetilde{A}_{2} \succ 0 \\
\left(a_{1} a_{2}, a_{2} \alpha_{1}-a_{1} \beta_{2}, a_{2} \beta_{1}-a_{1} \alpha_{2}\right)_{L R} & , \text { where } \widetilde{A}_{1} \prec 0 & , \widetilde{A}_{2} \succ 0 \\
\left(a_{1} a_{2}, a_{1} \alpha_{2}-a_{2} \beta_{1}, a_{1} \beta_{2}-a_{2} \alpha_{1}\right)_{L R} & , \text { where } \widetilde{A}_{1} \succ 0 & , \widetilde{A}_{2} \prec 0 \\
\left(a_{1} a_{2},-a_{1} \beta_{2}-a_{2} \beta_{1},-a_{1} \alpha_{2}-a_{2} \alpha_{1}\right)_{L R} & , \text { where } \widetilde{A}_{1} \prec 0 & , & \widetilde{A}_{2} \prec 0
\end{array}\right.
\end{aligned}
$$

Remark 1 . We denote the set of all $L R$-fuzzy numbers by $\mathcal{F}(\mathbb{R})$.

Definition 4. A linear ranking function is a function $\mathfrak{R}: \mathcal{F}(\mathbb{R}) \rightarrow \mathbb{R}$, which maps each fuzzy number into a real line, where there is a natural order.

$$
\Re(\widetilde{A})=\frac{1}{2} \int_{0}^{1}\left(L_{h}(x)+R_{h}(x)\right) d h
$$

where $\left[L_{h}(x), R_{h}(x)\right]$ is $h$ - cute of $\widetilde{A}$.

Let $\widetilde{A}=(a, \alpha, \beta)$ be a triangular fuzzy number then

$$
\mathfrak{R}(\widetilde{A})=a+\frac{1}{4}(\beta-\alpha) .
$$

\section{PLR fuzzy number}

Here a very useful form of fuzzy numbers is discussed and the arithmetic definition of this form of fuzzy numbers is given. We also show that the arithmetic of this form of fuzzy numbers is simple when compared with the current form of fuzzy numbers.

Definition 5. (PLR fuzzy number) Let

$$
\widetilde{A}=(a, \alpha, \beta)_{L R}, \quad \alpha, \beta \geq 0 \text { and } a \neq 0
$$

be the $L R$ fuzzy number, then

$$
\widetilde{A}=(a, \widehat{\alpha} \%, \widehat{\beta} \%), \quad \widehat{\alpha}, \widehat{\beta} \geq 0 \text { and } a \neq 0
$$


is called $P L R$ fuzzy number and the $\widehat{\alpha}$ and $\widehat{\beta}$ are the left and right percentage deviation from the center of fuzzy numbers $(a)$, respectively. The formula to convert $L R$ fuzzy number to $P L R$ fuzzy numbers and back are as follows :

$$
\begin{aligned}
& \widetilde{A}=(a, \alpha, \beta)_{L R}=\left(a, \frac{\alpha}{|a|} 100 \%, \frac{\beta}{|a|} 100 \%\right), \\
& \widetilde{A}=(a, \alpha \%, \beta \%)=\left(a, \frac{\alpha|a|}{100}, \frac{\beta|a|}{100}\right)_{L R} .
\end{aligned}
$$

Note that, $L R$ fuzzy numbers and $P L R$ fuzzy number are called normal and percentage form of fuzzy numbers respectively.

Example 1. Suppose $\widetilde{A}=(10,2,3)_{L R}$ is a $L R$ fuzzy number then its $P L R$ form is as follow:

$$
\widetilde{A}=(10,2,3)_{L R}=\left(10, \frac{2}{|10|} 100 \%, \frac{3}{|10|} 100 \%\right)=(10,20 \%, 30 \%) .
$$

Furthermore suppose $\widetilde{A}=(-50,8 \%, 14 \%)$ is a $P L R$ fuzzy number then its $L R$ form is as follow:

$$
\widetilde{A}=(-50,8 \%, 14 \%)=\left(-50, \frac{8 \times|-50|}{100}, \frac{14 \times|-50|}{100}\right)_{L R}=(-50,4,7)_{L R} .
$$

Definition 6. Suppose $\widetilde{A}=(a, \alpha \%, \beta \%)$ is $P L R$ fuzzy number, if $\alpha=\beta$ then $\widetilde{A}$ is symmetric $P L R$ fuzzy number and we show $\widetilde{A}=(a, \alpha \%)$.

\section{Arithmetic Operation on PLR Fuzzy Numbers}

Let $\widetilde{A}_{1}=\left(a_{1}, \alpha_{1} \%, \beta_{1} \%\right)$ and $\widetilde{A}_{2}=\left(a_{2}, \alpha_{2} \%, \beta_{2} \%\right)$ denote $P L R$ fuzzy numbers and $r \in \mathbb{R},(r \neq 0)$ then $L R$ form of $\widetilde{A}_{1}$ and $\widetilde{A}_{2}$ are as follows:

$$
\widetilde{A}_{1}=\left(a_{1}, \frac{\alpha_{1}\left|a_{1}\right|}{100}, \frac{\beta_{1}\left|a_{1}\right|}{100}\right)_{L R}, \quad \widetilde{A}_{2}=\left(a_{2}, \frac{\alpha_{2}\left|a_{2}\right|}{100}, \frac{\beta_{2}\left|a_{2}\right|}{100}\right)_{L R} .
$$

Addition $(\oplus)$ : According to Equation (1),we obtain

$$
\begin{aligned}
\widetilde{A}_{1} \oplus \widetilde{A}_{2} & =\left(a_{1}+a_{2}, \frac{\alpha_{1}\left|a_{1}\right|}{100}+\frac{\alpha_{2}\left|a_{2}\right|}{100}, \frac{\beta_{1}\left|a_{1}\right|}{100}+\frac{\beta_{2}\left|a_{2}\right|}{100}\right)_{L R} \\
& =\left(a_{1}+a_{2}, \frac{\alpha_{1}\left|a_{1}\right|+\alpha_{2}\left|a_{2}\right|}{100}, \frac{\beta_{1}\left|a_{1}\right|+\beta_{2}\left|a_{2}\right|}{100}\right)_{L R} .
\end{aligned}
$$

$P L R$ form of above $L R$ fuzzy number is as follow:

$$
\begin{aligned}
\widetilde{A}_{1} \oplus \widetilde{A}_{2} & =\left(a_{1}+a_{2}, \frac{\alpha_{1}\left|a_{1}\right|+\alpha_{2}\left|a_{2}\right|}{100} \times \frac{100 \%}{\left|a_{1}+a_{2}\right|}, \frac{\beta_{1}\left|a_{1}\right|+\beta_{2}\left|a_{2}\right|}{100} \times \frac{100 \%}{\left|a_{1}+a_{2}\right|}\right) \\
& =\left(a_{1}+a_{2}, \frac{\alpha_{1}\left|a_{1}\right|+\alpha_{2}\left|a_{2}\right|}{\left|a_{1}+a_{2}\right|} \%, \frac{\beta_{1}\left|a_{1}\right|+\beta_{2}\left|a_{2}\right|}{\left|a_{1}+a_{2}\right|} \%\right) .
\end{aligned}
$$

Scalar Multiplication:

Case 1: If $r>0$ as a result $|r a|=r|a|$ therefore

$$
r \widetilde{A}=\left(r a, r \frac{\alpha|a|}{100}, r \frac{\beta|a|}{100}\right)_{L R} .
$$

$P L R$ form of above $L R$ fuzzy number is as follow:

$$
\begin{aligned}
r \widetilde{A} & =\left(r a, r \frac{\alpha|a|}{100} \times \frac{100 \%}{|r a|}, \frac{r \beta|a|}{100} \times \frac{100 \%}{|r a|}\right) \\
& =\left(r a, r \frac{\alpha|a|}{100} \times \frac{100 \%}{r|a|}, \frac{r \beta|a|}{100} \times \frac{100 \%}{r|a|}\right)=(r a, \alpha \%, \beta \%) .
\end{aligned}
$$

Case 2: If $r<0$, similar to Case 1 we have

$$
r \widetilde{A}=(r a, \beta \%, \alpha \%) .
$$




\section{Multiplication:}

Case 1: Let $\widetilde{A}_{1}$ and $\widetilde{A}_{2}$ be non-negative $P L R$ fuzzy numbers then $\left|a_{1}\right|=a_{1},\left|a_{2}\right|=a_{2}$ and $\left|a_{1} a_{2}\right|=a_{1} a_{2}$. According to Equations (2), we have

$$
\begin{aligned}
\widetilde{A}_{1} \otimes \widetilde{A}_{2} & =\left(a_{1} a_{2}, \frac{\alpha_{1} a_{1}}{100} a_{2}+\frac{\alpha_{2} a_{2}}{100} a_{1}, \frac{\beta_{1} a_{1}}{100} a_{2}+\frac{\beta_{2} a_{2}}{100} a_{1}\right)_{L R} \\
& =\left(a_{1} a_{2}, \frac{a_{1} a_{2}\left(\alpha_{1}+\alpha_{2}\right)}{100}, \frac{a_{1} a_{2}\left(\beta_{1}+\beta_{2}\right)}{100}\right)_{L R} .
\end{aligned}
$$

$P L R$ form of above $L R$ fuzzy number is as follow:

$$
\begin{aligned}
\widetilde{A}_{1} \otimes \widetilde{A}_{2} & =\left(a_{1} a_{2}, \frac{a_{1} a_{2}\left(\alpha_{1}+\alpha_{2}\right)}{100} \times \frac{100}{\left|a_{1} a_{2}\right|} \%, \frac{a_{1} a_{2}\left(\beta_{1}+\beta_{2}\right)}{100} \times \frac{100}{\left|a_{1} a_{2}\right|} \%\right) \\
\Rightarrow \widetilde{A}_{1} \otimes \widetilde{A}_{2} & =\left(a_{1} a_{2},\left(\alpha_{1}+\alpha_{2}\right) \%,\left(\beta_{1}+\beta_{2}\right) \%\right) .
\end{aligned}
$$

Case 2: Let $\widetilde{A}_{1}=\left(a_{1}, \alpha_{1} \%, \beta_{1} \%\right)$ and $\widetilde{A}_{2}=\left(a_{2}, \alpha_{2} \%, \beta_{2} \%\right)$ be non-positive $P L R$ fuzzy numbers then

$$
\widetilde{A}_{1} \otimes \widetilde{A}_{2}=\left(a_{1} a_{2},\left(\beta_{1}+\beta_{2}\right) \%,\left(\alpha_{1}+\alpha_{2}\right) \%\right) .
$$

Case 3: Let $\widetilde{A}_{1}=\left(a_{1}, \alpha_{1} \%, \beta_{1} \%\right)$ is non-negative and $\widetilde{A}_{2}=\left(a_{2}, \alpha_{2} \%, \beta_{2} \%\right)$ be non-positive PLR fuzzy numbers then

$$
\widetilde{A}_{1} \otimes \widetilde{A}_{2}=\left(a_{1} a_{2},\left(\alpha_{2}+\beta_{1}\right) \%,\left(\alpha_{1}+\beta_{2}\right) \%\right) .
$$

Case 4: Let $\widetilde{A}_{1}=\left(a_{1}, \alpha_{1} \%, \beta_{1} \%\right)$ is non-positive and $\widetilde{A}_{2}=\left(a_{2}, \alpha_{2} \%, \beta_{2} \%\right)$ be non-negative PLR fuzzy numbers then

$$
\widetilde{A}_{1} \otimes \widetilde{A}_{2}=\left(a_{1} a_{2},\left(\alpha_{1}+\beta_{2}\right) \%,\left(\alpha_{2}+\beta_{1}\right) \%\right)
$$

In summing up, if $\widetilde{A}_{1}=\left(a_{1}, \alpha_{1} \%, \beta_{1} \%\right)$ and $\widetilde{A}_{2}=\left(a_{2}, \alpha_{2} \%, \beta_{2} \%\right)$ be $P L R$ fuzzy numbers then

$$
\begin{aligned}
& \widetilde{A}_{1} \oplus \widetilde{A}_{2}=\left(a_{1}+a_{2}, \frac{\alpha_{1}\left|a_{1}\right|+\alpha_{2}\left|a_{2}\right|}{\left|a_{1}+a_{2}\right|} \%, \frac{\beta_{1}\left|a_{1}\right|+\beta_{2}\left|a_{2}\right|}{\left|a_{1}+a_{2}\right|} \%\right) \\
& r \widetilde{A}= \begin{cases}(r a, \alpha \%, \beta \%) & , \quad r>0 \\
(r a, \beta \%, \alpha \%) & , \quad r<0\end{cases} \\
& \widetilde{A}_{1} \otimes \widetilde{A}_{2}=\left\{\begin{array}{lll}
\left(a_{1} a_{2},\left(\alpha_{1}+\alpha_{2}\right) \%,\left(\beta_{1}+\beta_{2}\right) \%\right) & , \widetilde{A}_{1} \succeq 0, & \widetilde{A}_{2} \succeq 0 \\
\left(a_{1} a_{2},\left(\alpha_{1}+\beta_{2}\right) \%,\left(\alpha_{2}+\beta_{1}\right) \%\right) & , \widetilde{A}_{1} \preceq 0, & \widetilde{A}_{2} \succeq 0 \\
\left(a_{1} a_{2},\left(\alpha_{2}+\beta_{1}\right) \%,\left(\alpha_{1}+\beta_{2}\right) \%\right) & , \widetilde{A}_{1} \succeq 0, & \widetilde{A}_{2} \preceq 0 \\
\left(a_{1} a_{2},\left(\beta_{1}+\beta_{2}\right) \%,\left(\alpha_{1}+\alpha_{2}\right) \%\right) & , \widetilde{A}_{1} \preceq 0, & \widetilde{A}_{2} \preceq 0
\end{array}\right.
\end{aligned}
$$

Proposition 1. Let $\widetilde{A}_{1}=\left(a_{1}, \alpha_{1} \%, \beta_{1} \%\right), \widetilde{A}_{2}=\left(a_{2}, \alpha_{2} \%, \beta_{2} \%\right), \ldots, \widetilde{A}_{n}=\left(a_{n}, \alpha_{n} \%, \beta_{n} \%\right)$ be PLR fuzzy numbers and

$$
\begin{aligned}
& P=\left\{i \mid \widetilde{A}_{i} \succ 0, \quad i=1,2, \ldots, n\right\} \\
& N=\left\{i \mid \widetilde{A}_{i} \prec 0, \quad i=1,2, \ldots, n\right\}
\end{aligned}
$$

then

$$
\widetilde{A}_{1} \otimes \widetilde{A}_{2} \otimes \cdots \otimes \widetilde{A}_{n}=\left(a_{1} a_{2} \cdots a_{n},\left(\sum_{i \in P} \alpha_{i}+\sum_{i \in N} \beta_{i}\right) \%,\left(\sum_{i \in N} \alpha_{i}+\sum_{i \in P} \beta_{i}\right) \%\right)
$$


Proposition 2. Let $\widetilde{A}_{1}=\left(a_{1}, \alpha_{1} \%, \beta_{1} \%\right), \widetilde{A}_{2}=\left(a_{2}, \alpha_{2} \%, \beta_{2} \%\right), \ldots, \widetilde{A}_{n}=\left(a_{n}, \alpha_{n} \%, \beta_{n} \%\right)$ be non-negative $P L R$ fuzzy numbers then

$$
\widetilde{A}_{1} \otimes \widetilde{A}_{2} \otimes \cdots \otimes \widetilde{A}_{n}=\left(a_{1} a_{2} \cdots a_{n},\left(\alpha_{1}+\alpha_{2}+\cdots+\alpha_{n}\right) \%,\left(\beta_{1}+\beta_{2}+\cdots+\beta_{n}\right) \%\right) .
$$

Proposition 3. Let $\widetilde{A}_{1}=\left(a_{1}, \alpha_{1} \%, \beta_{1} \%\right), \widetilde{A}_{2}=\left(a_{2}, \alpha_{2} \%, \beta_{2} \%\right), \ldots, \widetilde{A}_{n}=\left(a_{n}, \alpha_{n} \%, \beta_{n} \%\right)$ be non-positive $P L R$ fuzzy numbers then

$$
\widetilde{A}_{1} \otimes \widetilde{A}_{2} \otimes \cdots \otimes \widetilde{A}_{n}=\left(a_{1} a_{2} \cdots a_{n},\left(\beta_{1}+\beta_{2}+\cdots+\beta_{n}\right) \%,\left(\alpha_{1}+\alpha_{2}+\cdots+\alpha_{n}\right) \%\right) .
$$

Example 2. Let $\widetilde{A}_{1}=(4,2 \%, 3 \%), \widetilde{A}_{2}=(-5,8 \%, 4 \%), \widetilde{A}_{3}=(6,9 \%, 5 \%), \widetilde{A}_{4}=(3,2 \%, 8 \%)$ and $\widetilde{A}_{5}=(-3,1 \%, 6 \%)$ be $P L R$ fuzzy numbers. Then $P=\left\{i \mid \widetilde{A}_{i} \succ 0\right\}=\{1,3,4\}$ and $N=\left\{i \mid \widetilde{A}_{i} \prec 0\right\}=$ $=\{2,5\}$. Multiplying these fuzzy numbers is calculated as follows:

$$
\begin{aligned}
& \widetilde{A}_{1} \otimes \widetilde{A}_{2} \otimes \widetilde{A}_{3} \otimes \widetilde{A}_{4} \otimes \widetilde{A}_{5} \\
& =\left(4 \times(-5) \times 6 \times 3 \times(-3),\left[\sum_{i \in\{1,3,4\}} \alpha_{i}+\sum_{i \in\{2,5\}} \beta_{i}\right] \%,\left[\sum_{i \in\{2,5\}} \alpha_{i}+\sum_{i \in\{1,3,4\}} \beta_{i}\right] \%\right) \\
& =(1080,[(2+9+2)+(4+6)] \%,[(8+1)+(3+5+8)] \%) \\
& =(1080,23 \%, 25 \%) .
\end{aligned}
$$

According to Proposition 1, it is observed that multiplication of fuzzy numbers by $P L R$ fuzzy numbers is simple and uncomplicated in contrast to $L R$ fuzzy numbers.

\section{Predicting fuzzy optimal solution}

In this section, we describe how the maximum variation range of optimal solution $\left(\widetilde{Z}^{*}\right)$ can be predicted respectively for general form of fuzzy linear programming and special form of fuzzy linear programming problem when the maximum variation range of fuzzy variable is predetermined.

Proposition 4. Let $\widetilde{A}_{1}=\left(a_{1}, \alpha_{1} \%, \beta_{1} \%\right)$ and $\widetilde{A}_{2}=\left(a_{2}, \alpha_{2} \%, \beta_{2} \%\right)$ be $P L R$ fuzzy numbers and $a_{1} a_{2}>0$ then

$$
\widetilde{A}_{1} \oplus \widetilde{A}_{2}=(\widehat{a}, \widehat{\alpha} \%, \widehat{\beta} \%)
$$

then

$$
\begin{aligned}
& \min \left\{\alpha_{1}, \alpha_{2}\right\} \leq \widehat{\alpha} \leq \max \left\{\alpha_{1}, \alpha_{2}\right\} \\
& \min \left\{\beta_{1}, \beta_{2}\right\} \leq \widehat{\beta} \leq \max \left\{\beta_{1}, \beta_{2}\right\}
\end{aligned}
$$

Proof. Without prejudice to the generality of the problem suppose $\alpha_{1} \leq \alpha_{2}$ as a result $\max \left\{\alpha_{1}, \alpha_{2}\right\}=\alpha_{2}$ and $\min \left\{\alpha_{1}, \alpha_{2}\right\}=\alpha_{1}$. In addition,

$$
a_{1} a_{2}>0 \Rightarrow\left|a_{1}+a_{2}\right|=\left|a_{1}\right|+\left|a_{2}\right| \neq 0
$$

then

$$
\begin{aligned}
& \alpha_{1} \leq \alpha_{2} \\
\Rightarrow & \alpha_{1}\left|a_{1}\right| \leq \alpha_{2}\left|a_{1}\right| \\
\Rightarrow & \alpha_{1}\left|a_{1}\right|+\alpha_{2}\left|a_{2}\right| \leq \alpha_{2}\left|a_{1}\right|+\alpha_{2}\left|a_{2}\right| \\
\Rightarrow & \alpha_{1}\left|a_{1}\right|+\alpha_{2}\left|a_{2}\right| \leq \alpha_{2}\left(\left|a_{1}\right|+\left|a_{2}\right|\right)=\alpha_{2}\left|a_{1}+a_{2}\right| \\
\Rightarrow & \frac{\alpha_{1} a_{1}+\alpha_{2} a_{2}}{\left|a_{1}+a_{2}\right|} \leq \alpha_{2}=\max \left\{\alpha_{1}, \alpha_{2}\right\} \\
\Rightarrow & \widehat{\alpha} \leq \max \left\{\alpha_{1}, \alpha_{2}\right\}
\end{aligned}
$$




$$
\begin{aligned}
& \alpha_{2} \geq \alpha_{1} \\
\Rightarrow & \alpha_{2}\left|a_{2}\right| \geq \alpha_{1}\left|a_{2}\right| \\
\Rightarrow & \alpha_{1}\left|a_{1}\right|+\alpha_{2}\left|a_{2}\right| \geq \alpha_{1}\left|a_{1}\right|+\alpha_{1}\left|a_{2}\right| \\
\Rightarrow & \alpha_{1}\left|a_{1}\right|+\alpha_{2}\left|a_{2}\right| \geq \alpha_{1}\left(\left|a_{1}\right|+\left|a_{2}\right|\right)=\alpha_{1}\left|a_{1}+a_{2}\right| \\
\Rightarrow & \frac{\alpha_{1} a_{1}+\alpha_{2} a_{2}}{\left|a_{1}+a_{2}\right|} \geq \alpha_{1}=\min \left\{\alpha_{1}, \alpha_{2}\right\} \\
\Rightarrow & \widehat{\alpha} \geq \min \left\{\alpha_{1}, \alpha_{2}\right\}
\end{aligned}
$$

$$
\text { (3) and (4) } \Rightarrow \min \left\{\alpha_{1}, \alpha_{2}\right\} \leq \widehat{\alpha} \leq \max \left\{\alpha_{1}, \alpha_{2}\right\} \text {. }
$$

Proof for $\widehat{\beta}$ is similar to the proof for $\widehat{\alpha}$.

Proposition 5. Let $\widetilde{A}_{1}=\left(a_{1}, \alpha_{1} \%, \beta_{1} \%\right), \widetilde{A}_{2}=\left(a_{2}, \alpha_{2} \%, \beta_{2} \%\right), \ldots, \widetilde{A}_{n}=\left(a_{n}, \alpha_{n} \%, \beta_{n} \%\right)$ be PLR fuzzy numbers and $a_{i} a_{j}>0, i \neq j$ then

$$
\widetilde{A}_{1} \oplus \widetilde{A}_{2} \oplus \cdots \oplus \widetilde{A}_{n}=\left(a_{1}+a_{2}+\cdots+a_{n}, \widehat{\alpha} \%, \widehat{\beta} \%\right)
$$

then

$$
\min \left\{\alpha_{1}, \alpha_{2}, \ldots, \alpha_{n}\right\} \leq \widehat{\alpha} \leq \max \left\{\alpha_{1}, \alpha_{2}, \ldots, \alpha_{n}\right\}
$$

and

$$
\min \left\{\beta_{1}, \beta_{2}, \ldots, \beta_{n}\right\} \leq \widehat{\beta} \leq \max \left\{\beta_{1}, \beta_{2}, \ldots, \beta_{n}\right\}
$$

Predicting maximum variation range of objective function

Now we show how the maximum variation range of fuzzy objective function is predicted for general form of fully fuzzy linear programming problems.

Let a fully fuzzy linear programming be defined as follow:

$$
\begin{aligned}
& \begin{array}{l}
\text { Min } \\
\operatorname{Max}
\end{array} \quad \widetilde{Z}=\sum_{j=1}^{n} \widetilde{c}_{j} \otimes \widetilde{x}_{j} \\
& \text { s.t. } \quad \sum_{j=1}^{n} \widetilde{a}_{i j} \otimes \widetilde{x}_{j} \begin{array}{l}
\underset{\text { or }}{\approx} \\
\text { or }
\end{array} \quad \widetilde{b}_{i} \quad, \quad i=1,2, \ldots, m \\
& \widetilde{x}_{j} \succeq 0
\end{aligned}
$$

where $\widetilde{x}_{j}=\left(x_{j}, \alpha_{x_{j}} \%, \beta_{x_{j}} \%\right), \widetilde{c}_{j}=\left(c_{j}, \alpha_{c_{j}} \%, \beta_{c_{j}} \%\right), \widetilde{a}_{i j}=\left(a_{i j}, \alpha_{a_{i j}} \%, \beta_{a_{i j}} \%\right)$ and $\widetilde{b}_{i}=\left(b_{i}, \alpha_{b_{i}} \%, \beta_{b_{i}} \%\right)$ be $P L R$ fuzzy numbers for $i=1, \ldots, m, j=1, \ldots, n$.

Theorem 1. Let $\widetilde{c}_{j}=\left(c_{j}, \alpha_{c_{j}} \%, \beta_{c_{j}} \%\right)$ and $\widetilde{x}_{j}=\left(x_{j}, \alpha_{x_{j}} \%, \beta_{x_{j}} \%\right), j=1,2, \ldots, n$ be non-negative $P L R$ fuzzy numbers and $\widetilde{x}_{j}$ be feasible solution of fully fuzzy linear programming problem (5) also

$$
\widetilde{Z}=(\widehat{z}, \widehat{\alpha} \%, \widehat{\beta} \%)=\sum_{j=1}^{n} \widetilde{c}_{j} \otimes \widetilde{x}_{j}
$$

is the objective function of fully fuzzy linear programming problem (32) then

$$
\widehat{z}=c_{1} x_{1}+c_{2} x_{2}+\cdots+c_{n} x_{n}
$$

and

$$
\alpha_{\min } \leq \widehat{\alpha} \leq \alpha_{\max }, \quad \beta_{\min } \leq \widehat{\beta} \leq \beta_{\max }
$$


where

$$
\begin{aligned}
& \alpha_{\text {min }}=\min \left\{\alpha_{c_{i}}+\alpha_{x_{i}} \mid i=1,2, \ldots, n\right\} \\
& \alpha_{\text {max }}=\max \left\{\alpha_{c_{i}}+\alpha_{x_{i}} \mid i=1,2, \ldots, n\right\} \\
& \beta_{\text {min }}=\min \left\{\beta_{c_{i}}+\beta_{x_{i}} \mid i=1,2, \ldots, n\right\} \\
& \beta_{\text {max }}=\max \left\{\beta_{c_{i}}+\beta_{x_{i}} \mid i=1,2, \ldots, n\right\}
\end{aligned}
$$

Theorem 2. Let $\widetilde{c}_{j}=\left(c_{j}, \alpha_{c_{j}} \%, \beta_{c_{j}} \%\right)$ and $\widetilde{x}_{j}=\left(x_{j}, \alpha_{x_{j}} \%, \beta_{x_{j}} \%\right), j=1,2, \ldots, n$ be non-negative $P L R$ fuzzy numbers as well as $\alpha_{x_{j}} \in\left[0, s_{j}\right]$ and $\beta_{x_{j}} \in\left[0, r_{j}\right]$ also

$$
\widetilde{Z}=(\widehat{z}, \widehat{\alpha} \%, \widehat{\beta} \%)=\sum_{j=1}^{n} \widetilde{c}_{j} \otimes \widetilde{x}_{j}
$$

is the objective function of fully fuzzy linear programming problem (5) then

$$
\begin{aligned}
& \min _{j=1,2, \ldots, n}\left\{\alpha_{c_{j}}\right\} \leq \widehat{\alpha} \leq \max _{j=1,2, \ldots, n}\left\{s_{j}+\alpha_{c_{j}}\right\} \\
& \min _{j=1,2, \ldots, n}\left\{\beta_{c_{j}}\right\} \leq \widehat{\beta} \leq \max _{j=1,2, \ldots, n}\left\{r_{j}+\beta_{c_{j}}\right\}
\end{aligned}
$$

Proof.

$$
\begin{aligned}
& \forall j=1, \ldots, n, \alpha_{x_{j}} \in\left[0, s_{j}\right] \text { and } \beta_{x_{j}} \in\left[0, r_{j}\right] \Rightarrow 0 \leq \alpha_{x_{j}} \leq s_{j} \quad \text { and } 0 \leq \beta_{x_{j}} \leq r_{j} \\
& \Rightarrow \forall j=1,2, \ldots, n \quad, \quad \alpha_{c_{j}} \leq \alpha_{x_{j}}+\alpha_{c_{j}} \leq s_{j}+\alpha_{c_{j}} \quad, \quad \beta_{c_{j}} \leq \beta_{x_{j}}+\beta_{c_{j}} \leq r_{j}+\beta_{c_{j}}
\end{aligned}
$$

So

$$
\begin{aligned}
\min _{j=1, \ldots, n}\left\{\alpha_{c_{j}}\right\} & \leq \min _{j=1, \ldots, n}\left\{\alpha_{x_{j}}+\alpha_{c_{j}}\right\} \\
\max _{j=1, \ldots, n}\left\{\alpha_{x_{j}}+\alpha_{c_{j}}\right\} & \leq \max _{j=1, \ldots, n}\left\{s_{j}+\alpha_{c_{j}}\right\} \\
\min _{j=1, \ldots, n}\left\{\beta_{c_{j}}\right\} & \leq \min \left\{\beta_{x_{j}}+\beta_{c_{j}}\right\} \\
\max _{j=1, \ldots, n}\left\{\beta_{x_{j}}+\beta_{c_{j}}\right\} & \leq \max _{j=1, \ldots, n}\left\{r_{j}+\beta_{c_{j}}\right\}
\end{aligned}
$$

According to Theorem 1 and above inequalities we obtain

$$
\begin{array}{r}
\min _{j=1,2, \ldots, n}\left\{\alpha_{c_{j}}\right\} \leq \widehat{\alpha} \leq \max _{j=1,2, \ldots, n}\left\{s_{j}+\alpha_{c_{j}}\right\} \\
\min _{j=1,2, \ldots, n}\left\{\beta_{c_{j}}\right\} \leq \widehat{\beta} \leq \max _{j=1,2, \ldots, n}\left\{r_{j}+\beta_{c_{j}}\right\}
\end{array}
$$

Example 3. Consider the following fully fuzzy linear programming problem:

$$
\begin{array}{cl}
\text { Min } \quad \widetilde{Z}= & \widetilde{c}_{1} \otimes \widetilde{x}_{1} \oplus \widetilde{c}_{2} \otimes \widetilde{x}_{2} \\
\text { s.t. } & \widetilde{a}_{11} \otimes \widetilde{x}_{1} \oplus \widetilde{a}_{12} \otimes \widetilde{x}_{2} \succeq \widetilde{b}_{1} \\
& \widetilde{a}_{21} \otimes \widetilde{x}_{1} \oplus \widetilde{a}_{22} \otimes \widetilde{x}_{2} \preceq \widetilde{b}_{2} \\
& \widetilde{x}_{1}, \widetilde{x}_{2} \succeq 0
\end{array}
$$

where $\widetilde{c}_{1}=(12,6 \%, 4 \%), \quad \widetilde{c}_{2}=(20,5 \%, 5 \%), \quad \widetilde{a}_{11}=(8,5 \%, 4 \%), \quad \widetilde{a}_{12}=(10,5 \%, 4 \%), \quad \widetilde{a}_{21}=(15,10 \%, 8 \%)$, $\widetilde{a}_{22}=(18,6 \%, 5 \%), \widetilde{b}_{1}=(60,10 \%, 6 \%), \widetilde{b}_{2}=(120,6 \%, 8 \%), \widetilde{x}_{1}=\left(x_{1}, \alpha_{x_{1}} \%, \beta_{x_{1}} \%\right)$ and $\widetilde{x}_{2}=\left(x_{2}, \alpha_{x_{2}} \%, \beta_{x_{2}} \%\right)$ be PLR fuzzy numbers. An addition maximum variation range of $\widetilde{x}_{1}$ and $\widetilde{x}_{2}$ are predetermined in Table 1.

Maximum variation range of $\widetilde{x}_{1}$ and $\widetilde{x}_{2}$

\begin{tabular}{lcclcc}
\hline & \multicolumn{2}{c}{$\widetilde{x}_{1}$} & & \multicolumn{2}{c}{$\widetilde{x}_{2}$} \\
\cline { 2 - 3 } \cline { 5 - 6 } & $s_{1}$ & $r_{1}$ & & $s_{2}$ & $r_{2}$ \\
\hline Maximum variation range & $6 \%$ & $4 \%$ & & $3 \%$ & $4 \%$ \\
\hline
\end{tabular}


Therefor

$$
\begin{aligned}
0 \leq \alpha_{x_{1}} \leq s_{1} & \Rightarrow \alpha_{c_{1}} \leq \alpha_{x_{1}}+\alpha_{c_{1}} \leq s_{1}+\alpha_{c_{1}} \\
0 \leq \alpha_{x_{2}} \leq s_{2} & \Rightarrow 6 \leq \alpha_{x_{1}}+\alpha_{c_{1}} \leq s_{1}+6=6+6=12 \\
& \Rightarrow 5 \leq \alpha_{c_{2}} \leq \alpha_{x_{2}}+\alpha_{c_{2}} \leq s_{2}+\alpha_{c_{2}} \\
0 \leq \beta_{x_{1}} \leq r_{1} & \Rightarrow \beta_{c_{1}} \leq \beta_{x_{1}}+\beta_{c_{1}} \leq r_{1}+\beta_{c_{1}} \\
& \Rightarrow 4 \leq \beta_{x_{1}}+\beta_{c_{1}} \leq r_{1}+4=4+8=8 \\
0 \leq \beta_{x_{2}} \leq r_{2} & \Rightarrow \beta_{c_{2}} \leq \beta_{x_{2}}+\beta_{c_{2}} \leq r_{2}+\beta_{c_{2}} \\
& \Rightarrow 5 \leq \beta_{x_{2}}+\beta_{c_{2}} \leq r_{2}+5=4+5=9
\end{aligned}
$$

Thus

$$
\begin{aligned}
& \min \left\{\alpha_{c_{1}}+\alpha_{x_{1}}, \alpha_{c_{2}}+\alpha_{x_{2}}\right\} \geq \min \{6,5\}=5 \\
& \max \left\{\alpha_{c_{1}}+\alpha_{x_{1}}, \alpha_{c_{2}}+\alpha_{x_{2}}\right\} \leq \max \{12,8\}=12
\end{aligned}
$$

and

$$
\begin{aligned}
& \min \left\{\beta_{c_{1}}+\beta_{x_{1}}, \beta_{c_{2}}+\beta_{x_{2}}\right\} \geq \min \{4,5\}=4 \\
& \max \left\{\beta_{c_{1}}+\beta_{x_{1}}, \beta_{c_{2}}+\beta_{x_{2}}\right\} \leq \max \{8,9\}=9
\end{aligned}
$$

According to Theorem 1 we find

$$
5 \leq \widehat{\alpha} \leq 12, \quad 4 \leq \widehat{\beta} \leq 9
$$

where $\widetilde{x}_{1}$ and $\widetilde{x}_{2}$ are feasible solutions and $\widetilde{Z}=(\widehat{z}, \widehat{\alpha} \%, \widehat{\beta} \%)$ is a value of objective function proportional to $\widetilde{x}_{1}$ and $\widetilde{x}_{2}$. The variation range of $\widehat{\alpha}$ and $\widehat{\beta}$ indicate that, for each fuzzy feasible solutions, $\widehat{\alpha}$ vary between $5 \%$ and $12 \%$ and $\widehat{\beta}$ vary between $4 \%$ and $9 \%$ (See Figure 1 ). Especially, if $\widetilde{x}_{1}^{*}$ and $\widetilde{x}_{2}^{*}$ are optimal feasible solutions then variation range of $\widetilde{Z}^{*}$ can be predicted as follows:

$$
\widetilde{Z}^{*}=\left\{\left(z^{*}, \alpha^{*} \%, \beta^{*} \%\right) \mid \alpha^{*} \in[5,12], \beta^{*} \in[4,9]\right\}
$$

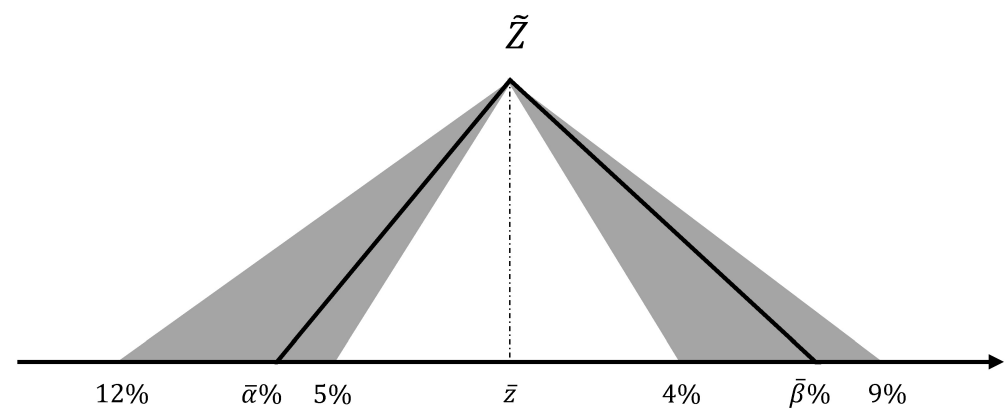

Figure 1. The variation range of left and right side of $\widetilde{Z}$

Note that Theorem 2 imply that the maximum variation range of left and right side of $\widetilde{Z}^{*}$ may be changed if the maximum variation range of left and right side of fuzzy variable is changed.

In other words:

- The minimum variation range of left and right side of $\widetilde{Z}^{*}$ is independent of fuzzy variables' variation range.

- The maximum variation range of left and right side of $\widetilde{Z}^{*}$ is dependent on fuzzy variables' variation range.

\section{Predicting optimal value of objective function}

Here, we describe how the optimal value of objective function in fully fuzzy linear programming problems are predictable when all of the parameters and variables are symmetric triangular fuzzy numbers. 
Theorem 3. If $\widetilde{A}=(a, \alpha \%)$ and $\widetilde{B}=(b, \beta \%)$ are symmetric $P L R$ fuzzy number and $a+b \neq 0$, then $\widetilde{A} \oplus \widetilde{B}$ is symmetric $P L R$ fuzzy number.

Proof. $\widetilde{A}=(a, \alpha \%)$ and $\widetilde{B}=(b, \beta \%)$ are symmetric $P L R$ fuzzy number, then we can show $\widetilde{A}=(a, \alpha \%, \alpha \%)$ and $\widetilde{B}=(b, \beta \%, \beta \%)$ and we have

$$
\widetilde{A} \oplus \widetilde{B}=\left(a+b, \frac{\alpha|a|+\beta|b|}{|a+b|} \%, \frac{\alpha|a|+\beta|b|}{|a+b|} \%\right) .
$$

Shows that $\widetilde{A} \oplus \widetilde{B}$ is symmetric $P L R$ fuzzy number.

Theorem 4. If $\widetilde{A}=(a, \alpha \%)$ and $\widetilde{B}=(b, \beta \%)$ are symmetric $P L R$ fuzzy number and $\widetilde{A}, \widetilde{B} \not 0$, then $\widetilde{A} \otimes \widetilde{B}$ is symmetric $P L R$ fuzzy number.

Proof. Analogous to the proof of Theorem 3.

Lemma 1. Let $\widetilde{A}_{j}=\left(a_{j}, \alpha_{j} \%\right)$ and $\widetilde{B}_{j}=\left(b_{j}, \beta_{j} \%\right), j=1,2, \ldots, n$ be non-negative symmetric $P L R$ fuzzy numbers and

$$
\widetilde{Z}=\sum_{j=1}^{n} \widetilde{A}_{j} \otimes \widetilde{B}_{j}
$$

then $\widetilde{Z}$ is symmetric $P L R$ fuzzy numbers.

Lemma 2. If $\widetilde{A}=(a, \alpha \%)$ is symmetric triangular fuzzy number then $\mathfrak{R}(\widetilde{A})=a$.

Suppose all the parameters and variables in the fully fuzzy linear programming (5) are triangular symmetric fuzzy numbers, then as a consequence of Lemma 1 the result of $\sum_{j=1}^{n} \widetilde{c}_{j} \otimes \widetilde{x}_{j}$ and $\sum_{j=1}^{n} \widetilde{a}_{i j} \otimes \widetilde{x}_{j}$ are symmetric triangular fuzzy numbers. In addition

$$
\begin{array}{ll}
\mathfrak{R}\left(\sum_{j=1}^{n} \widetilde{c}_{j} \otimes \widetilde{x}_{j}\right)=\sum_{j=1}^{n} c_{j} x_{j} & \\
\mathfrak{R}\left(\sum_{j=1}^{n} \widetilde{a}_{i j} \otimes \widetilde{x}_{j}\right)=\sum_{j=1}^{n} a_{i j} x_{j}, & i=1,2, \ldots, m \\
\mathfrak{R}\left(\widetilde{b}_{i}\right)=b_{i}, & i=1,2, \ldots, m \\
\mathfrak{R}\left(\widetilde{x}_{j}\right)=x_{j}, & j=1,2, \ldots, n
\end{array}
$$

In fact, the left and right sides of $P L R$ fuzzy parameters and variables are ignored by applying defuzzification. Hence the fully fuzzy linear programming (5) with using ranking function is converted to crisp linear programming as follows:

$$
\begin{aligned}
& \underset{(\operatorname{Max})}{\operatorname{Min}} \quad Z=\sum_{j=1}^{n} c_{j} x_{j} \\
& \text { s.t. } \quad \sum_{j=1}^{n} a_{i j} x_{j} \stackrel{\geq}{=} b_{i} \quad, \quad i=1,2, \ldots, m \\
& x_{j} \geq 0
\end{aligned}
$$

Assume that $x_{j}^{*}, j=1,2, \ldots, n$ are optimal feasible solutions of linear programming $(6)$ then $\widetilde{Z^{*}}=\left(z^{*}, \alpha^{*} \%\right)$ is a prediction for the optimal objective function of model (5) where

$$
z^{*}=c_{1} x_{1}^{*}+c_{2} x_{2}^{*}+\cdots+c_{n} x_{n}^{*}
$$

and

$$
\min _{j=1, \ldots, n}\left\{\alpha_{c_{j}}\right\} \leq \alpha^{*} \leq \max _{j=1, \ldots, n}\left\{\alpha_{c_{j}}+s_{j}\right\} .
$$

Example 4. A company produces 3 kinds of goods with high profitability. Market estimation shows that each of these products can be sold at $\$ 865, \$ 580$ and $\$ 470$ with maximum variation rate of $9 \%, 7.5 \%$ and $12 \%$ 
respectively. The company has 3 stages of production and each of these products require a proportion of the capacity of these stages. Maximum capacity of each production stages and proportion of the needed capacity for each product with its' maximum variation rate are given in Table 2.

Table 2

Require a portion of the capacity of production stages for each products

\begin{tabular}{|c|c|c|c|c|c|c|}
\hline \multirow[b]{2}{*}{ Products } & \multicolumn{2}{|c|}{ Production Stage 1} & \multicolumn{2}{|c|}{ Production Stage 2} & \multicolumn{2}{|c|}{ Production Stage 3} \\
\hline & Value & $\alpha$ & Value & $\alpha$ & Value & $\alpha$ \\
\hline P1 & 1 & $0 \%$ & 5 & $3 \%$ & 3 & $1 \%$ \\
\hline P2 & 0.8 & $0 \%$ & 3 & $5 \%$ & 2 & $2 \%$ \\
\hline P3 & 2 & $0 \%$ & 2.5 & $4 \%$ & 1.2 & $4 \%$ \\
\hline Maximum Capacity & 200 & $2 \%$ & 680 & $6 \%$ & 400 & $3 \%$ \\
\hline
\end{tabular}

$\alpha$ : Maximum variation rate of each data

Since these goods are all best-selling, the company wants to get maximum production capacity. Here the manager really wants to know the amount of maximum profit and variation rate of profit when maximum variation rate of products is predetermined to be $5 \%$ and $10 \%$ respectively. This problem of goods production in the way that profit is maximized and the restrictions are met, is formulated as the following fully fuzzy linear programming.

$$
\begin{aligned}
\operatorname{Max} \widetilde{Z}= & (865,9 \%) \otimes \widetilde{x}_{1} \oplus(580,7.5 \%) \otimes \widetilde{x}_{2} \oplus(470,12 \%) \otimes \widetilde{x}_{3} \\
\text { s.t. } \quad & (1,0 \%) \otimes \widetilde{x}_{1} \oplus(0.8,0 \%) \otimes \widetilde{x}_{2} \oplus(2,0 \%) \otimes \widetilde{x}_{3} \preceq(200,2 \%) \\
& (5,3 \%) \otimes \widetilde{x}_{1} \oplus(3,5 \%) \otimes \widetilde{x}_{2} \oplus(2.5,4 \%) \otimes \widetilde{x}_{3} \preceq(680,6 \%) \\
& (3,1 \%) \otimes \widetilde{x}_{1} \oplus(2,2 \%) \otimes \widetilde{x}_{2} \oplus(1.2,4 \%) \otimes \widetilde{x}_{3} \preceq(400,3 \%) \\
& \widetilde{x}_{1}, \widetilde{x}_{2}, \widetilde{x}_{3} \succeq 0
\end{aligned}
$$

By appealing to the ranking function for defuzzification, the above fully fuzzy linear programming is converted to simple crisp linear programming as shown by the following

$$
\begin{array}{cr}
\operatorname{Max} \quad Z= & 865 x_{1}+580 x_{2}+470 x_{3} \\
\text { s.t. } & x_{1}+0.8 x_{2}+2 x_{3} \leq 200 \\
& 5 x_{1}+3 x_{2}+2.5 x_{3} \leq 680 \\
& 3 x_{1}+2 x_{2}+1.2 x_{3} \leq 400 \\
& x_{1}, x_{2}, x_{3} \geq 0
\end{array}
$$

\begin{tabular}{|c|c|c|c|c|c|}
\hline & \multicolumn{3}{|c|}{ Maximum variation range of products } & \multirow{2}{*}{$\begin{array}{l}\text { Maximum variation } \\
\text { range of profit }\end{array}$} & \multirow[t]{2}{*}{$\widetilde{Z}^{*}$} \\
\hline & $\mathrm{P} 1\left(x_{1}\right)$ & $\mathrm{P} 2\left(x_{2}\right)$ & P3 $\left(x_{3}\right)$ & & \\
\hline 1 & $5 \%$ & $5 \%$ & $5 \%$ & $17 \%$ & $(2520,17 \%)$ \\
\hline 2 & $10 \%$ & $10 \%$ & $10 \%$ & $22 \%$ & $(2520,22 \%)$ \\
\hline
\end{tabular}

The maximum value of objective function of linear programming problem (8) is ( $\left.Z^{*}\right)$ equal to 2520 . Since fully fuzzy linear programming problem (7) is simplified to crisp linear programming problem $(8), Z^{*}=2520$ is an appropriate approximation for optimal profit irrespective of variation range of fuzzy parameters and fuzzy variables. Then, by applying Theorem 2 , according to maximum variation range of products, maximum variation rate of optimal profit is calculated, as given in Table 3.

Maximum variation range of products and profit for Example 4

\section{Conclusion}

The given approach of in this paper differs significantly from other similar research studies which were conducted in the class of fuzzy linear programming problems. Here we introduced the PLR fuzzy numbers which are applicable to many branches of knowledge. In addition, in contrast to arithmetic operations on $L R$ fuzzy numbers which are usually very complicated and voluminous, we have developed $P L R$ fuzzy numbers with simpler multiplication operations and fewer computational procedures. Therefor in this method we are 
able to reduce the lengthy numerical algorithms required in an arithmetic operations on fuzzy numbers when the number of multiplication operations are more than the number of additional operations. Furthermore, by applying our percentage form of fuzzy numbers in fuzzy linear programming problem, we are able to predict maximum variation range of optimal value of objective function when the maximum variation range of variables are predetermined.

\section{References}

1 Nehi, H. M., Maleki, H. R., \& Mashinchi, M. (2004). Solving fuzzy number linear programming problem by lexicographic ranking function. Italian Journal of Pure and Applied Mathematics, 15, 9-20.

2 Tanaka, H., Okuda, T., \& Asai, K. (1973). On fuzzy-mathematical programming. Journal of Cybernetics, 3, 37-46.

3 Bellman, R. E., \& Zadeh, L. A. (1970). Decision-making in a fuzzy environment. Management Science, $17,141-164$.

4 Zimmermann, H.-J. (1978). Fuzzy programming and linear programming with several objective functions. Fuzzy Sets and Systems, 1, 45-55.

5 Campos, L., \& Verdegay, J. (1989). Linear programming problems and ranking of fuzzy numbers. Fuzzy Sets and Systems, 32, 1-11.

6 Ebrahimnejad, A., Nasseri, S.H., Lotfi, F. H., \& Soltanifar, M. (2010). A primal-dual method for linear programming problems with fuzzy variables. European Journal of Industrial Engineering, 4, 189-209.

7 Ganesan, K., \& Veeramani, P. (2006). Fuzzy linear programs with trapezoidal fuzzy numbers. Annals of Operations Research, 143, 305-315.

8 Mahdavi-Amiri, N., \& Nasseri, S. (2006). Duality in fuzzy number linear programming by use of a certain linear ranking function. Applied Mathematics and Computation, 180, 206-216.

9 Mahdavi-Amiri, N., \& Nasseri, S. (2007). Duality results and a dual simplex method for linear programming problems with trapezoidal fuzzy variables. Fuzzy Sets and Systems, 158, 1961-1978.

10 Maleki, H., Tata, M., \& Mashinchi, M. (2000). Linear programming with fuzzy variables. Fuzzy Sets and Systems, 109, 21-33.

11 Chen, L.H., \& Ko, W.C. (2009). Fuzzy linear programming models for new product design using QFD with FMEA. Applied Mathematical Modelling, 33, 633-647.

12 Gupta, A., Kumar, A., \& Sharma, M. (2013). Applications of fuzzy linear programming with generalized lr flat fuzzy parameters. Fuzzy Information and Engineering, 5, 475-492.

13 Ebrahimnejad, A., \& Tavana, M. (2014). A novel method for solving linear programming problems with symmetric trapezoidal fuzzy numbers. Applied Mathematical Modelling, 38, 4388-4395.

14 Dong, J.-y., \& Wan, S.-P. (2018). A new trapezoidal fuzzy linear programming method considering the acceptance degree of fuzzy constraints violated. Knowledge-Based Systems, 148, 100-114.

15 Allahviranloo, T., Lotfi, F. H., Kiasary, M. K., Kiani, N. A., \& Alizadeh, L. (2008). Solving fully fuzzy linear programming problem by the ranking functhion. Applied Matematical Sciences, 2, 19-32.

16 Buckley, J. J., \& Feuring, T. (2000). Evolutionary algorithm solution to fuzzy problems: Fuzzy linear programming. Fuzzy Sets and Systems, 109, 35-53.

17 Hashemi, S. M., Modarres, M., Nasrabadi, E., \& Nasrabadi, M. M. (2006). Fully fuzzified linear programming, solution and duality. J. Intell. Fuzzy Syst., 17, 253-261.

18 Kumar, A., Kaur, J., \& Singh, P. (2010). Fuzzy optimal solution of fully fuzzy linear programming problems with inequality constraints. International Journal of Mathematical and Computer Sciences, 6, $37-41$.

19 Kumar, A., Kaur, J., \& Singh, P. (2011). A new method for solving fully fuzzy linear programming problems. Applied Mathematical Modelling, 35, 817-823.

20 Lotfi, F. H., Allahviranloo, T., Jondabeh, M. A., \& Alizadeh, L. (2009). Solving a full fuzzy linear programming using lexicography method and fuzzy approximate solution. Applied Mathematical Modelling, 33, 3151-3156.

21 Kaur, J., \& Kumar, A. (2013). Mehar's method for solving fully fuzzy linear programming problems with L-R fuzzy parameters. Applied Mathematical Modelling, 37, 7142-7153. 
22 Cheng, H., Huang, W., \& Cai, J. (2013). Method for solving fully fuzzy linear programming problems using deviation degree measure. Journal of Systems Engineering and Electronics, 24, 793-799.

23 Chehlabi, M., \& Allahviranloo, T. (2018). Positive or negative solutions to first-order fully fuzzy linear differential equations under generalized differentiability. Applied Soft Computing, 70, 359-370.

24 Najafi, H. S., Edalatpanah, S., \& Dutta, H. (2016). A nonlinear model for fully fuzzy linear programming with fully unrestricted variables and parameters. Alexandria Engineering Journal, 55, 2589-2595.

25 Dubois, D., \& Prade, H. (1980). Systems of linear fuzzy constraints. Fuzzy Sets and Systems, 3, 37-48.

26 Yager, R. R. (1981). A procedure for ordering fuzzy subsets of the unit interval. Information Sciences, 24, 143-161.

\author{
С.М. Давуди, Н.А. Абдул Рахман
}

\title{
Анықемес сызықтық бағдарламалау есебіндегі оңтайлы шешімді болжау
}

\begin{abstract}
Мақалада анықемес сандардың пайдалы түрі мен оның арифметикасы болып табылатын, анықемес сандардың LR пайыздық түрін анықтауға тырысқан. Авторлар анықемес сандардың осы формасын қолдана отырып, анықемес объективті функцияның оңтайлы мәнінің өзгеруінің максималды диапазонын қалай болжауға болатындығын көрсеткен. Анықемес мәселелер әдетте күрделі түрде шешілетіндіктен, бұл зерттеудің мақсаты - менеджер мәселелерді шешпестен оңтайлы шешім болжамына $\left(Z^{*}\right)$ қол жеткізе алатындай етіп түпкілікті шешім үшін болжам түрін шығару болып табылады. Соңында, анықемес сызықтық бағдарламалау кезінде анықемес объективті функцияның оңтайлы мәні анықемес айнымалының өзгеруінің максималды диапазоны алдын-ала анықталған кезде болжанады.
\end{abstract}

Kiлm сөздер: анықемес сандардың пайыздық түрі, тақ сан, анықемес сызықтық бағдарламалау, анықемес арифметика.

\author{
C.M. Давуди, Н.А. Абдул Рахман
}

\section{Прогнозирование оптимального решения в задаче нечеткого линейного программирования}

В статье предпринята попытка определить процентную форму LR нечетких чисел, которая является полезной формой нечетких чисел и ее арифметикой. Авторы показывают, как можно предсказать максимальный диапазон изменения оптимального значения нечеткой целевой функции с помощью этой формы нечетких чисел. Поскольку нечеткие проблемы, как правило, решаются сложным образом, цель этого исследования - выпустить своего рода прогноз для окончательного решения таким образом, чтобы менеджер мог получить доступ к прогнозу оптимального решения $\left(Z^{*}\right)$, не решая проблемы. Наконец, оптимальное значение нечеткой целевой функции при нечетком линейном программировании предсказывается, когда предопределен максимальный диапазон изменения нечеткой переменной.

Ключевые слова: процентная форма нечетких чисел, нечеткое число, нечеткое линейное программирование, нечеткая арифметика. 


\author{
M.T. Jenaliyev ${ }^{1}$, M.I. Ramazanov ${ }^{2, *}$, A.O. Tanin ${ }^{2}$ \\ ${ }^{1}$ Institute of Mathematics and Mathematical Modeling, Almaty, Kazakhstan; \\ ${ }^{2}$ Buketov Karaganda University, Karaganda, Kazakhstan \\ (E-mail: muvasharkhan@gmail.com,ramamur@mail.ru,tanin.alibek@gmail.com)
}

\title{
To the solution of the Solonnikov-Fasano problem with boundary moving on arbitrary law $x=\gamma(t)$.
}

\begin{abstract}
In this paper we study the solvability of the boundary value problem for the heat equation in a domain that degenerates into a point at the initial moment of time. In this case, the boundary changing with time moves according to an arbitrary law $x=\gamma(t)$. Using the generalized heat potentials, the problem under study is reduced to a pseudo-Volterra integral equation such that the norm of the integral operator is equal to one and it is shown that the corresponding homogeneous integral equation has a nonzero solution.

Key words: heat equation, moving boundary, degenerating domain, pseudo-Volterra integral equation.
\end{abstract}

\section{Introduction}

In many practically important engineering problems, the process of forming a temperature field in the structure under study is accompanied by the removal of a part of the substance from the surface, which leads to a change in its boundaries over time. The need to take into account the of the boundaries mobility of the studied domain significantly complicates the solution of the corresponding problems [1-5].

For example, in mathematical modeling of thermophysical processes in an electric arc of high-current disconnecting devices, the heat equation is used, which takes into account the effect of heat sources in the arc and the effect of contracting the axial section of the arc in the cathode region into the contact spot [6]. Moreover, the diameter of the contact spot is much less than the section diameter of the developed column of the arc, which makes it possible to consider it as a mathematical point. At the initial moment of time, the contacts are in a closed state and solution domain of the problem is absent; then, the solution domain changes over time according to the conditions for opening the contacts.

From a mathematical point of view, the singularity of the problem under consideration lies, firstly, in the presence of a moving boundary, and secondly, in the degeneration of the solution domain at the initial moment $[7,8]$. Problems in domains with moving boundaries are also relevant in modeling physical processes in a gas discharge plasma, during melting of electrical contacts, the effect of an electric arc on contacts, in studying the problems of thermal shock in domains with a moving boundary, in solving a number of problems in hydromechanics [9-13].

Applying the method of generalized heat potentials, a number of similar problems can be reduced to the solution of sinqular Volterra type integral equations of the second kind. It is essential here that if in the boundary value problem the variable domain does not degenerate into a point at the initial moment of time, then the integral equation equivalent to it is solved by the method of successive approximations. If the domain degenerates into a point at the initial moment of time, then the integral equation of the boundary value problem has a singularity, which is that the integral from the kernel tends to unity as the upper limit of integration tends to the lower one, and this means that the method of successive approximations is not applicable to it.

\section{Statement of the problem}

Let's study the solvability issues of the following boundary value problem:

$$
\frac{\partial u}{\partial t}-a^{2} \frac{\partial^{2} u}{\partial x^{2}}=f(x, t), \quad\{0<x<\gamma(t), t>0\}
$$

\footnotetext{
${ }^{*}$ Corresponding author.
}

E-mail:ramamur@mail.ru 


$$
\left.\frac{\partial u}{\partial x}\right|_{x=0}=u_{0}(t), \quad \frac{d \tilde{u}(t)}{d t}+\left.\frac{\partial u}{\partial x}\right|_{x=\gamma(t)}=u_{1}(t)
$$

where $\tilde{u}(t)=u(\gamma(t), t), \quad \gamma(0)=0$ for $\gamma(t)=\left[t\left(1+\alpha_{0}(t)\right)\right]^{\omega}, \quad \omega>\frac{1}{2}$.

Function $\gamma(t):(0, \infty) \rightarrow(0, \infty)$ satisfies the following conditions:

1. asymptotics of the function $\gamma(t)$ as $t \rightarrow 0$ and as $t \rightarrow \infty$ has the form $t^{\omega}$, where $\omega>\frac{1}{2}$;

2. starting from some moment of time $t_{1}^{*}$ until moment of time $t_{2}^{*}$ the function $\gamma(t)$ is arbitrary, strictly monotone and one-to-one, i.e. there is a reverse transformation $\gamma^{-1}(t)$.

We introduce the classes of solutions and data of the problem as follows:

$$
\begin{gathered}
\left(x+[\gamma(t)]^{\frac{3}{2 \omega}-1}\right)^{-1} u(x, t) \in L_{\infty}(G), \text { i.e. } \quad u(x, t) \in L_{\infty}\left(G ;\left(x+[\gamma(t)]^{3 / 2 \omega-1}\right)^{-1}\right), \\
f(x, t) \in W_{\infty}^{1,0}\left(G ;[\gamma(t)]^{3 / 2 \omega-1} \exp \left\{[\gamma(t)]^{\frac{2 \omega-1}{\omega}} /\left(4 a^{2}\right)\right\}\right) ; \\
u_{0}(t) \in L_{\infty}\left(R_{+} ;[\gamma(t)]^{-(3 / 2 \omega-1)}\right) ; \quad u_{1}(t) \in L_{\infty}\left(R_{+} ;[\gamma(t)]^{3 / 2 \omega-1}\right) .
\end{gathered}
$$

This kind of boundary value problem (1) arises, for example, in studies of the Stefan problem [14].

\section{Transformation of problem (1) and reduction of it to the integral equation}

Introducing a new unknown function $v(x, t)=\frac{\partial u}{\partial x}$, we transform problem (1)-(2) to the next problem:

$$
\begin{gathered}
\frac{\partial v}{\partial t}-a^{2} \frac{\partial^{2} v}{\partial x^{2}}=\tilde{f}(x, t), \quad\{0<x<t, \quad t>0\} \\
\left.v(x, t)\right|_{x=0}=v_{0}(t),\left.\quad\left(\frac{\partial v}{\partial x}+\frac{1+\gamma^{\prime}(t)}{a^{2}} v\right)\right|_{x=\gamma(t)}=v_{1}(t),
\end{gathered}
$$

where $\tilde{f}(x, t) \equiv \frac{\partial f(x, t)}{\partial x}, \quad v_{0}(t) \equiv u_{0}(t), \quad v_{1}(t) \equiv \frac{u_{1}(t)}{a^{2}}+\left.\frac{f(x, t)}{a^{2}}\right|_{x=\gamma(t)}$.

Remark 1. Each solution to boundary value problem (3)-(4) defines a unique solution (up to a constant) of boundary value problem (1)-(2).

We will find the solution of problem (3)-(4) as the sum of heat potentials [15]:

$$
\begin{aligned}
& v(x, t)=\frac{1}{2 a \sqrt{\pi}} \int_{0}^{t} \int_{0}^{\infty} \frac{1}{(t-\tau)^{1 / 2}} \exp \left\{-\frac{(x-\xi)^{2}}{4 a^{2}(t-\tau)}\right\} \tilde{f}(\xi, \tau) d \xi d \tau+ \\
& +\frac{1}{4 a^{3} \sqrt{\pi}} \int_{0}^{t} \frac{x}{(t-\tau)^{3 / 2}} \exp \left\{-\frac{x^{2}}{4 a^{2}(t-\tau)}\right\} \nu(\tau) d \tau+ \\
& +\frac{1}{2 a \sqrt{\pi}} \int_{0}^{t} \frac{1}{(t-\tau)^{1 / 2}} \exp \left\{-\frac{(x-\gamma(\tau))^{2}}{4 a^{2}(t-\tau)}\right\} \varphi(\tau) d \tau .
\end{aligned}
$$

The function defined by equality (5) satisfies the equation (3) for any functions $\nu(t)$ and $\varphi(t)$, which are still unknown and are to be determined further.

Satisfying solution (5) with boundary conditions (4), we obtain the following integral equation:

$$
\varphi(t)+\int_{0}^{t} K_{\gamma}(t, \tau) \varphi(\tau) d \tau=F(t)
$$

kernel $K_{\gamma}(t, \tau)$ of which can be represented as a sum:

$$
K_{\gamma}(t, \tau)=\sum_{i=1}^{4} K_{\gamma}^{(i)}(t, \tau),
$$


where:

$$
\begin{aligned}
& K_{\gamma}^{(1)}=\frac{1}{2 a \sqrt{\pi}} \frac{\gamma(t)+\gamma(\tau)}{(t-\tau)^{\frac{3}{2}}} \exp \left\{-\frac{(\gamma(t)+\gamma(\tau))^{2}}{4 a^{2}(t-\tau)}\right\} ; \\
& K_{\gamma}^{(2)}=-\frac{1}{2 a \sqrt{\pi}} \frac{\gamma(t)-\gamma(\tau)}{(t-\tau)^{\frac{3}{2}}} \exp \left\{-\frac{(\gamma(t)-\gamma(\tau))^{2}}{4 a^{2}(t-\tau)}\right\} ; \\
& K_{\gamma}^{(3)}=-\frac{1}{a \sqrt{\pi}} \frac{1+\gamma(t)^{\prime}}{(t-\tau)^{\frac{1}{2}}} \exp \left\{-\frac{(\gamma(t)+\gamma(\tau))^{2}}{4 a^{2}(t-\tau)}\right\} ; \\
& K_{\gamma}^{(3)}=\frac{1}{a \sqrt{\pi}} \frac{1+\gamma(t)^{\prime}}{(t-\tau)^{\frac{1}{2}}} \exp \left\{-\frac{(\gamma(t)-\gamma(\tau))^{2}}{4 a^{2}(t-\tau)}\right\} .
\end{aligned}
$$

The free term of equation (6) has the following form:

$$
\begin{gathered}
F(t)=-\frac{a}{\sqrt{\pi}} \int_{0}^{t}\left[\frac{1}{(t-\tau)^{3 / 2}}-\frac{\gamma^{2}(t)}{2 a^{2}(t-\tau)^{5 / 2}}\right] \exp \left\{-\frac{\gamma^{2}(t)}{4 a^{2}(t-\tau)}\right\} v_{0}(\tau) d \tau+ \\
-\frac{\left.1+\gamma^{\prime}(\mathrm{t})\right)}{a \sqrt{\pi}} \int_{0}^{t} \frac{\gamma(t)}{(t-\tau)^{3 / 2}} \exp \left\{-\frac{\gamma^{2}(t)}{4 a^{2}(t-\tau)}\right\} v_{0}(\tau) d \tau+2 a^{2} \cdot v_{1}(t)- \\
-\frac{1}{2 a \sqrt{\pi}} \int_{0}^{t} \int_{0}^{\infty}\left[\frac{\gamma(t)+\xi}{(t-\tau)^{3 / 2}} \exp \left\{-\frac{(\gamma(t)+\xi)^{2}}{4 a^{2}(t-\tau)}\right\}-\frac{\gamma(t)-\xi}{(t-\tau)^{3 / 2}} \exp \left\{-\frac{(\gamma(t)-\xi)^{2}}{4 a^{2}(t-\tau)}\right\}\right] \tilde{f}(\xi, \tau) d \xi d \tau- \\
-\frac{1}{a \sqrt{\pi}} \int_{0}^{t} \int_{0}^{\infty} \frac{1+\gamma^{\prime}(t)}{(t-\tau)^{1 / 2}} \cdot \exp \left\{-\frac{(\gamma(t)-\xi)^{2}}{4 a^{2}(t-\tau)}\right\} \cdot \tilde{f}(\xi, \tau) d \xi d \tau .
\end{gathered}
$$

We will find the solution of integral equation (6) in the class of functions:

$$
[\gamma(t)]^{\frac{3}{2 \omega}-1} \varphi(t) \in L_{\infty}(0, \infty) \text {, т.e. } \varphi(t) \in L_{\infty}\left(0, \infty ;[\gamma(t)]^{\frac{3}{2 \omega}-1}\right) .
$$

For convenience, we represent equation (6) as follows:

$$
\varphi_{1}(t)+\int_{0}^{t}\left[\frac{\gamma(t)}{\gamma(\tau)}\right]^{\frac{3}{2 \omega}-1} K_{\gamma}(t, \tau) \varphi_{1}(\tau) d \tau=F_{1}(t)
$$

where

$$
\varphi_{1}(t)=t^{\frac{3}{2 \omega}-1} \cdot \varphi(t), \quad F_{1}(t)=t^{\frac{3}{2 \omega}-1} \cdot F(t)
$$

Remark 2. ([16], p.183) If the (particular) solution of the integral equation

$$
y(x)+\int_{a}^{x} K(x, t) y(t) d t=f(x)
$$

is given by formula

$$
y(x)=f(x)+\int_{a}^{x} R(x, t) f(t) d t,
$$

then the (particular) solution of the integral equation (with a modified kernel)

$$
y(x)+\int_{a}^{x} K(x, t) \frac{g(x)}{g(t)} y(t) d t=f(x)
$$

is given by the formula

$$
y(x)=f(x)+\int_{a}^{x} R(x, t) \frac{g(x)}{g(t)} f(t) d t .
$$

The same is true for the solutions of the corresponding homogeneous equations.

Such kind of the Volterra integral equations were considered in the papers $[17,18]$.

Note that a feature of integral equation (7) is the following property of the kernel $K_{\gamma}(t, \tau)$ :

$$
\lim _{t \rightarrow 0+} \int_{0}^{t} K_{\gamma}(t, \tau) d \tau=1 .
$$

In order to solve integral equation (7), consider the corresponding characteristic integral equation. 
Characteristic integral equation. Estimates for kernels of integral operators

For integral equation (7) we will construct a characteristic equation

$$
\varphi(t)+\int_{0}^{t}\left[\frac{\gamma(t)}{\gamma(\tau)}\right]^{\frac{3}{2 \omega}-1} K_{h}(t, \tau) \varphi(\tau) d \tau=g(t),
$$

where

$$
\begin{aligned}
& K_{h}(t, \tau)=\sum_{i=1}^{4} K_{h}^{(i)}(t, \tau) \\
& K_{h}^{(1)}(t, \tau)=\frac{1}{2 a \sqrt{\pi}} \cdot \frac{(2 \omega-1)^{\frac{3}{2}}\left([\gamma(\tau)]^{\frac{2 \omega-1}{\omega}} \cdot[\gamma(t)]^{\frac{2 \omega-2}{\omega}}+[\gamma(t)]^{\frac{4 \omega-3}{\omega}}\right)\left\{[\gamma(\tau)]^{\frac{1}{\omega}}\right\}^{\prime}}{\left([\gamma(t)]^{\frac{2 \omega-1}{\omega}}-[\gamma(\tau)]^{\frac{2 \omega-1}{\omega}}\right)^{\frac{3}{2}}} . \\
& \cdot \exp \left\{-\frac{(2 \omega-1)\left([\gamma(t)]^{\frac{2 \omega-1}{\omega}}+[\gamma(\tau)]^{\frac{2 \omega-1}{\omega}}\right)^{2}}{4 a^{2}\left([\gamma(t)]^{\frac{2 \omega-1}{\omega}}-[\gamma(\tau)]^{\frac{2 \omega-1}{\omega}}\right)}\right\} \\
& K_{h}^{(2)}(t, \tau)=-\frac{1}{2 a \sqrt{\pi}} \cdot \frac{(2 \omega-1)^{\frac{3}{2}} \cdot[\gamma(t)]^{\frac{2 \omega-2}{\omega}} \cdot\left\{[\gamma(\tau)]^{\frac{1}{\omega}}\right\}^{\prime}}{\left(t^{2 \omega-1}-\tau^{2 \omega-1}\right)^{\frac{1}{2}}} \cdot \exp \left\{-\frac{(2 \omega-1)\left([\gamma(t)]^{\frac{2 \omega-1}{\omega}}-[\gamma(\tau)]^{\frac{2 \omega-1}{\omega}}\right)^{2}}{4 a^{2}\left([\gamma(t)]^{\frac{2 \omega-1}{\omega}}-[\gamma(\tau)]^{\frac{2 \omega-1}{\omega}}\right)}\right\} \\
& K_{h}^{(3)}(t, \tau)=-\frac{2}{2 a \sqrt{\pi}} \cdot \frac{(2 \omega-1)^{\frac{3}{2}} \cdot[\gamma(t)]^{\frac{2 \omega-2}{\omega}} \cdot\left\{[\gamma(\tau)]^{\frac{1}{\omega}}\right\}^{\prime}}{\left(t^{2 \omega-1}-\tau^{2 \omega-1}\right)^{\frac{1}{2}}} \cdot \exp \left\{-\frac{(2 \omega-1)\left([\gamma(t)]^{\frac{2 \omega-1}{\omega}}+[\gamma(\tau)]^{\frac{2 \omega-1}{\omega}}\right)^{2}}{4 a^{2}\left([\gamma(t)]^{\frac{2 \omega-1}{\omega}}-[\gamma(\tau)]^{\frac{2 \omega-1}{\omega}}\right)}\right\} \\
& K_{h}^{(4)}(t, \tau)=\frac{2}{a \sqrt{\pi}} \cdot \frac{(2 \omega-1)^{\frac{3}{2}} \cdot[\gamma(t)]^{\frac{2 \omega-2}{\omega}} \cdot\left\{[\gamma(\tau)]^{\frac{1}{\omega}}\right\}^{\prime}}{\left(t^{2 \omega-1}-\tau^{2 \omega-1}\right)^{\frac{1}{2}}} \cdot \exp \left\{-\frac{(2 \omega-1)\left([\gamma(t)]^{\frac{2 \omega-1}{\omega}}-[\gamma(\tau)]^{\frac{2 \omega-1}{\omega}}\right)^{2}}{4 a^{2}\left([\gamma(t)]^{\frac{2 \omega-1}{\omega}}-[\gamma(\tau)]^{\frac{2 \omega-1}{\omega}}\right)}\right\}
\end{aligned}
$$

Next, we show that it is indeed characteristic equation for the equation (7). Firstly, we note that the kernel $K_{h}(t, \tau)$ has the property:

$$
\lim _{t \rightarrow 0} \int_{0}^{t} K_{\gamma}^{(1)}(t, \tau) d \tau=1 .
$$

Equation (8), using the following the change of variables:

$$
\begin{aligned}
& \gamma(t)=\left(\frac{1}{2 \omega-1} \cdot t_{1}\right)^{\frac{\omega}{2 \omega-1}}, \quad \gamma(t)=\left(\frac{1}{2 \omega-1} \cdot \tau_{1}\right)^{\frac{\omega}{2 \omega-1}}, \\
& \varphi\left[\left(\frac{1}{2 \omega-1} \cdot t_{1}\right)^{\frac{\omega}{2 \omega-1}}\right]=\varphi_{1}\left(t_{1}\right), \quad g\left[\left(\frac{1}{2 \omega-1} \cdot t_{1}\right)^{\frac{\omega}{2 \omega-1}}\right]=g_{1}\left(t_{1}\right),
\end{aligned}
$$

reduces to the following integral equation [8]:

$$
\varphi_{1}\left(t_{1}\right)+\int_{0}^{t_{1}} \sqrt{\frac{t_{1}}{\tau_{1}}} K_{1}\left(t_{1}, \tau_{1}\right) \varphi_{1}\left(\tau_{1}\right) d \tau_{1}=g_{1}\left(t_{1}\right) .
$$

The kernel $K_{1}\left(t_{1}, \tau_{1}\right)$ has the form:

$$
K_{1}\left(t_{1}, \tau_{1}\right)=\sum_{i=1}^{4} K_{1}^{(i)}\left(t_{1}, \tau_{1}\right),
$$


where

$$
\begin{aligned}
& K_{1}^{(1)}\left(t_{1}, \tau_{1}\right)=\frac{1}{2 a \sqrt{\pi}} \cdot \frac{t_{1}+\tau_{1}}{\left(t_{1}-\tau_{1}\right)^{\frac{3}{2}}} \cdot \exp \left\{-\frac{\left(t_{1}+\tau_{1}\right)^{2}}{4 a^{2}\left(t_{1}-\tau_{1}\right)}\right\} ; \\
& K_{1}^{(2)}\left(t_{1}, \tau_{1}\right)=-\frac{1}{2 a \sqrt{\pi}} \cdot \frac{t_{1}-\tau_{1}}{\left(t_{1}-\tau_{1}\right)^{\frac{3}{2}}} \cdot \exp \left\{-\frac{\left(t_{1}-\tau_{1}\right)^{2}}{4 a^{2}\left(t_{1}-\tau_{1}\right)}\right\} ; \\
& K_{1}^{(3)}\left(t_{1}, \tau_{1}\right)=-\frac{2}{a \sqrt{\pi}} \cdot \frac{1}{\left(t_{1}-\tau_{1}\right)^{\frac{1}{2}}} \cdot \exp \left\{-\frac{\left(t_{1}+\tau_{1}\right)^{2}}{4 a^{2}\left(t_{1}-\tau_{1}\right)}\right\} ; \\
& K_{1}^{(4)}\left(t_{1}, \tau_{1}\right)=\frac{2}{a \sqrt{\pi}} \cdot \frac{1}{\left(t_{1}-\tau_{1}\right)^{\frac{1}{2}}} \cdot \exp \left\{-\frac{\left(t_{1}-\tau_{1}\right)^{2}}{4 a^{2}\left(t_{1}-\tau_{1}\right)}\right\}
\end{aligned}
$$

Solution of integral equation (9) has the form [8]:

$$
\varphi\left(t_{1}\right)=g\left(t_{1}\right)+\int_{0}^{t} \sqrt{\frac{t_{1}}{\tau_{1}}} \cdot R\left(t_{1}, \tau_{1}\right) \cdot g\left(t_{1}\right) d \tau_{1}+C \cdot \varphi_{\text {hom }}\left(t_{1}\right),
$$

which also belongs to the class $L_{\infty}\left(\mathrm{R}_{+} ; \sqrt{t_{1}} \exp \left\{\frac{t_{1}}{4 a^{2}}\right\}\right)$.

Moreover, the following Lemma holds for the resolvent [8].

Lemma 1. The resolvent $R\left(\mathrm{t}_{1}, \tau_{1}\right)$ admits an estimate

$$
\left|R\left(\mathrm{t}_{1}, \tau_{1}\right)\right| \leq C \frac{\tau_{1}}{\left(t_{1}-\tau_{1}\right)^{3 / 2}} \exp \left\{-\frac{t_{1} \tau_{1}}{a^{2}\left(t_{1}-\tau_{1}\right)}\right\}, \quad 0<\tau_{1}<t_{1}<+\infty .
$$

\section{Solution of characteristic integral equation (8)}

Returning to the old variables, in equality (10), we obtain the solution of characteristic equation (8):

$$
\varphi(t)=g(t)+\int_{0}^{t}\left(\frac{t}{\tau}\right)^{\frac{3}{2 \omega}-1} R_{h}(t, \tau) g(t) d \tau+C \cdot \varphi_{\mathrm{hom}}\left((2 \omega-1)(2 \omega-1)[\gamma(\tau)]^{\frac{2 \omega-1}{\omega}}\right),
$$

and the resolvent $R_{h}(t, \tau)$ satisfies the estimate

$$
R_{h}(t, \tau) \leq C_{1}(\omega) \cdot \frac{[\gamma(t)]^{\frac{5 \omega-3}{\omega}}\left\{[\gamma(\tau)]^{\frac{1}{\omega}}\right\}^{\prime}}{\left([\gamma(t)]^{\frac{2 \omega-1}{\omega}}-[\gamma(\tau)]^{\frac{2 \omega-1}{\omega}}\right)^{\frac{3}{2}}} \cdot \exp \left\{-\frac{(2 \omega-1)[\gamma(t)]^{\frac{2 \omega-1}{\omega}} \cdot[\gamma(\tau)]^{\frac{2 \omega-1}{\omega}}}{a^{2}\left([\gamma(t)]^{\frac{2 \omega-1}{\omega}}-[\gamma(\tau)]^{\frac{2 \omega-1}{\omega}}\right)}\right\} .
$$

Theorem 1. For any right side $g(t) \in L_{\infty}\left(R ;[\gamma(t)]^{\frac{3}{2 \omega}-1}\right)$ integral equation (8) has a general solution $\varphi(t) \in L_{\infty}\left(R ;[\gamma(t)]^{\frac{3}{2 \omega}-1}\right):$

$$
\varphi(t)=g(t)+\int_{0}^{t}\left(\frac{\gamma(t)}{\gamma(\tau)}\right)^{\frac{3}{2 \omega}-1} R_{h}(t, \tau) g(t) d \tau+C \cdot \varphi_{\mathrm{hom}}\left((2 \omega-1) t^{2 \omega-1}\right),
$$

where $\varphi_{\text {hom }}(t)$ is the solution of the homogeneous equation, and for the resolvent $R_{h}(t, \tau)$ we have estimate (11).

Solution of integral equation (7). (Regularization method for solving the characteristic equation)

Using Remark 2, we consider equation (6), which we represent as:

$$
\varphi(t)+\int_{0}^{t} K_{h}(t, \tau) \varphi(\tau) d \tau=\int_{0}^{t}\left[K_{h}(t, \tau)-K_{\gamma}(t, \tau)\right] \varphi(\tau) d \tau+F(t) .
$$


Assuming the right-hand side of equation (12) is temporarily known, we write its solution as:

$$
\begin{gathered}
\varphi(t)=\int_{0}^{t}\left[K_{h}(t, \tau)-K_{\gamma}(t, \tau)\right] \varphi(\tau) d \tau+\int_{0}^{t} R_{h}(t, \tau)\left\{\int_{0}^{\tau}\left[K_{h}\left(\tau, \tau_{1}\right)-K_{\gamma}\left(\tau, \tau_{1}\right)\right] \varphi\left(\tau_{1}\right) d \tau_{1}\right\} d \tau+ \\
+F(t)+\int_{0}^{t} R_{h}(t, \tau) \cdot F(\tau) d \tau+C_{0} \cdot \varphi_{\mathrm{hom}}\left((2 \omega-1) \cdot t^{2 \omega-1}\right) .
\end{gathered}
$$

In the iterated integral, we change the order of integration and change the roles of the variables $\tau$ and $\tau_{1}$, then we obtain

$$
\varphi(t)+\int_{0}^{t} \overline{\tilde{K}}(t, \tau) \cdot \varphi(\tau) d \tau=C_{0} \cdot \varphi_{\mathrm{hom}}\left((2 \omega-1) \cdot t^{2 \omega-1}\right)+\hat{F}(t)
$$

The kernel $\overline{\tilde{K}}(t, \tau)$ has the form:

$$
\overline{\tilde{K}}(t, \tau)=\tilde{K}(t, \tau)+\bar{K}(t, \tau)
$$

where

$$
\tilde{K}(t, \tau)=K_{h}(t, \tau)-K_{\gamma}(t, \tau), \quad \bar{K}(t, \tau)=\int_{\tau_{1}}^{t} R\left(t, \tau_{1}\right)\left[K_{h}\left(\tau_{1}, \tau\right)-K_{\gamma}\left(\tau_{1}, \tau\right)\right] d \tau_{1} .
$$

Let's introduce the following notations:

$$
K_{h}^{(i)}(t, \tau)=P_{h}^{(i)} \exp \left\{-Q_{h}^{(i)}\right\} ; \quad K_{\gamma}^{(i)}(t, \tau)=P_{\gamma}^{(i)} \exp \left\{-Q_{\gamma}^{(i)}\right\}, \quad i=1,2,3,4,
$$

where

$$
\begin{gathered}
P_{h}^{(1)}(t, \tau)=\frac{1}{2 a \sqrt{\pi}} \cdot \frac{(2 \omega-1)^{\frac{3}{2}}\left([\gamma(\tau)]^{\frac{2 \omega-1}{\omega}} \cdot[\gamma(t)]^{\frac{2 \omega-2}{\omega}}+[\gamma(t)]^{\frac{4 \omega-3}{\omega}}\right)\left\{[\gamma(\tau)]^{\frac{1}{\omega}}\right\}^{\prime}}{\left([\gamma(t)]^{\frac{2 \omega-1}{\omega}}-[\gamma(\tau)]^{\frac{2 \omega-1}{\omega}}\right)^{\frac{3}{2}}}, \\
Q_{h}^{(1)}(t, \tau)=\frac{(2 \omega-1)\left([\gamma(t)]^{\frac{2 \omega-1}{\omega}}+[\gamma(\tau)]^{\frac{2 \omega-1}{\omega}}\right)^{2}}{4 a^{2}\left([\gamma(t)]^{\frac{2 \omega-1}{\omega}}-[\gamma(\tau)]^{\frac{2 \omega-1}{\omega}}\right)} \\
P_{\gamma}^{(1)}(t, \tau)=\frac{1}{2 a \sqrt{\pi}} \cdot \frac{\gamma(t)+\gamma(\tau)}{(t-\tau)^{\frac{3}{2}}}, \quad Q_{\gamma}^{(1)}(t, \tau)=\frac{(\gamma(t)+\gamma(\tau))^{2}}{4 a^{2}(t-\tau)}
\end{gathered}
$$

Now we prove the following theorem.

Theorem 2. If function $\gamma(t)=\left[t\left(1+\alpha_{0}(t)\right)\right]^{\omega}$, where $\alpha_{0}(t)=t^{\beta} \sigma(t), \quad \beta>0$, and function $\sigma(t)$ is twice continuously differentiable for $0<t<\infty$, and $|\sigma(t)| \leq C, \sigma(t) \neq 0$, then we have an estimate:

$$
\left|K_{\gamma}(t, \tau)-K_{h}(t, \tau)\right| \leq C(\omega) \frac{t^{\omega-1}}{\sqrt{t-\tau}} \times\left[\exp \left(-Q_{\gamma}(t, \tau) / 2\right)+\exp \left(-Q_{h}(t, \tau) / 2\right)\right]
$$

and the limit relation:

$$
\lim _{t \rightarrow+0} \int_{0}^{t}\left[K_{\gamma}(t, \tau)-K_{h}(t, \tau)\right] d \tau=0
$$

holds.

Note that estimate (14) is obvious for the terms

$$
\left|K_{h}^{(i)}(t, \tau)-K_{\gamma}^{(i)}(t, \tau)\right| \text { for } i=2,3,4 .
$$

Now we prove the estimate (14) for $i=1$. 
Lemma 2. If $\alpha_{0}(t)$ is monotonically increasing function, then the inequalities:

$$
\begin{gathered}
2 \omega-1 \leq \frac{\left[t\left(1+\alpha_{0}(t)\right)\right]^{2 \omega-1}-\left[\tau\left(1+\alpha_{0}(\tau)\right)\right]^{2 \omega-1}}{\left[t\left(1+\alpha_{0}(t)\right)\right]^{2 \omega-2}\left(1+\alpha_{0}\left(t_{1}\right)+t_{1} \alpha_{0}^{\prime}\left(t_{1}\right)\right)(t-\tau)} \leq 1 \text { for } \frac{1}{2}<\omega<1, \\
1 \leq \frac{\left[t\left(1+\alpha_{0}(t)\right)\right]^{2 \omega-1}-\left[\tau\left(1+\alpha_{0}(\tau)\right)\right]^{2 \omega-1}}{\left[t\left(1+\alpha_{0}(t)\right)\right]^{2 \omega-2}\left(1+\alpha_{0}\left(t_{1}\right)+t_{1} \alpha_{0}^{\prime}\left(t_{1}\right)\right)(t-\tau)} \leq 2 \omega-1 \text { for } \omega \geq 1,
\end{gathered}
$$

hold, where $t_{1}=\tau+\theta_{1}(t-\tau), \quad 0<\theta_{1}<1$.

Proof of the Lemma 2. It's obvious that [20;456]:

$$
\begin{gathered}
2 \omega-1 \leq \frac{1-x^{2 \omega-1}}{1-x} \leq 1, \quad \text { if } \quad \frac{1}{2}<\omega<1, \quad 0 \leq x \leq 1 ; \\
1 \leq \frac{1-x^{2 \omega-1}}{1-x} \leq 2 \omega-1, \quad \text { if } \quad \omega \geq 1, \quad 0 \leq x \leq 1 .
\end{gathered}
$$

Let $\omega \geq 1$, then for $0 \leq \tau \leq t$ we get

$$
\begin{gathered}
{\left[t\left(1+\alpha_{0}(t)\right)\right]^{2 \omega-2}\left(t-\tau+t \alpha_{0}(t)-\tau \alpha_{0}(\tau)\right) \leq\left[t\left(1+\alpha_{0}(t)\right)\right]^{2 \omega-1}-\left[\tau\left(1+\alpha_{0}(\tau)\right)\right]^{2 \omega-1} \leq} \\
\leq(2 \omega-1)\left[t\left(1+\alpha_{0}(t)\right)\right]^{2 \omega-2}\left(t-\tau+t \alpha_{0}(t)-\tau \alpha_{0}(\tau)\right) .
\end{gathered}
$$

Using Lagrange's interpolation formula, we have

$$
t \alpha_{0}(t)-\tau \alpha_{0}(\tau)=\left(\alpha_{0}\left(t_{1}\right)+t_{1} \alpha_{0}^{\prime}\left(t_{1}\right)\right)(t-\tau),
$$

where $t_{1}=\tau+\theta_{1}(t-\tau), 0<\theta_{1}<1$.

The proof is analogously for the case $\frac{1}{2}<\omega<1$.

The following lemmas are proved in a similar way.

Lemma 3. If the function $\gamma(t)=\left[t\left(1+\alpha_{0}(t)\right)\right]^{\omega}$, where $\alpha_{0}(t)=t^{\beta} \sigma(t), \beta>0$ and the function $\alpha_{0}(t)$ increases monotonically for $0<t<\infty$, and $|\sigma(t)| \leq C$, then estimate:

$$
\left|P_{h}^{(1)}(t, \tau)-P_{\gamma}^{(1)}(t, \tau)\right| \leq C_{2}(\omega) \frac{t^{\omega+\beta}}{(t-\tau)^{\frac{3}{2}}}
$$

holds.

Lemma 4. Under the conditions of Lemma 3, estimate:

$$
\left|Q_{h}^{1}(t, \tau)-Q_{\gamma}^{1}(t, \tau)\right| \leq M_{1} \frac{t^{2 \omega+\beta}}{t-\tau}+M_{2} t^{2 \omega-1}
$$

holds.

Proof of the Theorem 2. First, we establish the following inequality:

$$
P_{2}(t, \tau)=\cdot \frac{\left[t\left(1+\alpha_{0}(t)\right)\right]^{\omega}}{2 \sqrt{\pi}(t-\tau)^{3 / 2}} \leq M_{3}(\omega) \cdot \frac{t^{\omega}}{(t-\tau)^{3 / 2}} .
$$

For those values of parameter $\omega, 0<\tau<t<\infty$, for which $\left|Q_{h}^{1}(t, \tau)-Q_{\gamma}^{1}(t, \tau)\right|>0$, the required estimate follows from the following inequalities:

$$
\begin{gathered}
\left|K_{h}^{1}(t, \tau)-K_{\gamma}^{1}(t, \tau)\right| \leq\left|\left(P_{h}^{1}(t, \tau)-P_{\gamma}^{1}(t, \tau)\right) \exp \left\{-Q_{\gamma}(t, \tau)\right\}\right|+ \\
+\left|P_{\gamma}^{1}(t, \tau) \exp \left\{-Q_{h}^{1}(t, \tau)\right\}\left(1-\exp \left\{-Q_{\gamma}^{1}(t, \tau)+Q_{h}^{1}(t, \tau)\right\}\right)\right| \leq \\
\leq\left|P_{h}^{1}(t, \tau)-P_{\gamma}^{1}(t, \tau)\right| \exp \left\{-Q_{\gamma}^{1}(t, \tau)\right\}+\left|P_{\gamma}^{1}(t, \tau)\left(Q_{\gamma}^{1}(t, \tau)-Q_{h}^{1}(t, \tau)\right) \exp \left\{-Q_{h}^{1}(t, \tau)\right\}\right| .
\end{gathered}
$$

Hence, taking into account the Lemmas $2-4$, we get:

$$
\left|K_{h}-K_{\gamma}\right| \leq\left\{\bar{M} \frac{t^{\omega+\beta}}{(t-\tau)^{\frac{3}{2}}}+M_{3} \frac{t^{\omega}}{(t-\tau)^{\frac{3}{2}}}\left(M_{1} \frac{t^{2 \omega+\beta}}{t-\tau}+M_{2} t^{2 \omega-1}\right)\right\} e^{-Q_{h}} \leq
$$




$$
\begin{gathered}
\leq \cdot \frac{t^{\omega-1}}{(t-\tau)^{\frac{1}{2}}}\left(\bar{M} \frac{t^{\beta+1}}{t-\tau}+\bar{M}_{1} \frac{t^{2 \omega+\beta+1}}{(t-\tau)^{2}}+\bar{M}_{2} \frac{t^{2 \omega}}{t-\tau}\right) \cdot e^{-Q_{h}} \leq \\
\leq \\
\frac{t^{\omega-1}}{\sqrt{t-\tau}}\left(\frac{t}{t-\tau} \cdot e^{\frac{-Q_{h}}{2}} \cdot \bar{M} t^{\beta}+\frac{t^{2}}{(t-\tau)^{2}} e^{\frac{-Q_{h}}{2}} \cdot \bar{M}_{1} t^{2 \omega+\beta-1}+\right. \\
\left.+\frac{t}{t-\tau} e^{\frac{-Q_{h}}{2}} \cdot \bar{M}_{2} t^{2 \omega-1}\right) e^{\frac{-Q_{h}}{2}} \leq C(\omega)\left[\frac{\alpha(t)}{\alpha(\tau)}\right]^{\frac{3}{2}-\omega} \cdot \frac{t^{\omega-1}}{\sqrt{t-\tau}} e^{\frac{-Q_{h}}{2}} .
\end{gathered}
$$

If the values of the parameter $\omega$ and $0<\tau<t<\infty$ are such that the difference $Q_{\gamma}(t, \tau)-Q_{h}(t, \tau)<0$, then it is enough in the same inequalities to interchange the functions $Q_{\gamma}(t, \tau)$ and $Q_{h}(t, \tau), P_{\gamma}(t, \tau)$ and $P_{h}(t, \tau)$ accordingly.

The validity of inequality (14) shows that the difference $K_{\gamma}(t, \tau)-K_{h}(t, \tau)$ has a weak singularity and the limit relation (15) holds.

$$
\lim _{t \rightarrow+0} \int_{0}^{t} \frac{t^{\omega-1}}{\sqrt{t-\tau}}\left[\exp \left(-Q_{h}(t, \tau) / 2\right)+\exp \left(-Q_{\gamma}(t, \tau) / 2\right)\right] d \tau=0 .
$$

Consequently, equation (8) is indeed characteristic equation for equation (7). Theorem 2 is proved.

In order to obtain estimates for $\bar{K}(t, \tau)$, and at the same time for $\overline{\tilde{K}}(t, \tau)$ estimate for the resolvent, we represent in the form:

$$
\left|R\left\{\frac{1}{2 \omega-1}\left([\alpha(\eta)]^{\frac{1-2 \omega}{\omega}}-[\alpha(t)]^{\frac{1-2 \omega}{\omega}}\right)\right\}\right| \leq \tilde{M}_{2} \frac{t^{3 / 2} \eta^{\frac{3}{2}(2 \omega-1)}}{(t-\eta)^{3 / 2}} \exp \left(-C_{2}(\omega) \frac{t \eta^{2 \omega-1}}{t-\eta}\right) .
$$

Here $C_{j}(\omega), \tilde{M}_{j}(\omega), j=1,2$ are constants depending only on $\omega$.

Using estimates (14) and (16), we obtain the following theorem:

Theorem 3. If function $\gamma(t)=\left[t\left(1+\alpha_{0}(t)\right)\right]^{\omega}$, where $\alpha_{0}(t)=t^{\beta} \sigma(t), \beta>0$, and the function $\sigma(t)$ is twice continuously differentiable for $0<t<\infty$, and $|\sigma(t)| \leq C, \sigma(t) \neq 0$, then the kernel $\tilde{K}(t, \tau)$ has a weak singularity, i.e. we have an estimate:

$$
|\overline{\tilde{K}}(t, \tau)| \leq \frac{t^{1 / 2+\varepsilon}}{\tau^{3 / 2-\omega+\varepsilon}(t-\tau)^{1 / 2}}, 0<\varepsilon<\omega-\frac{1}{2}, 0<\tau<t<\infty,
$$

which means that integral equation (7) for any $f(t),[\gamma(t)]^{\frac{3}{2 \omega-1}} \cdot f(t) \in L_{\infty}\left(R_{+}\right)$has a unique nonzero solution:

$$
\varphi(t) \in L_{\infty}\left(R_{\infty} ;[\gamma(t)]^{3 / 2 \omega-1}\right)
$$

Proof. Since $\overline{\tilde{K}}(t, \tau)=\tilde{K}(t, \tau)+\bar{K}(t, \tau)$, then estimate (17) follows from (14), estimates for resolvent (16) and below relations. Using the following double inequality $[19 ; 55]$ :

$$
C_{1} t^{\rho-1}(t-\tau) \leq t^{\rho}-\tau^{\rho} \leq C_{2} \tau^{\rho-1}(t-\tau) \text {, где } C_{1}=\min \{1, \rho\}, C_{2}=\max \{1, \rho\},
$$

first we get $(\rho=2 \omega-1)$ :

$$
\bar{K}(t, \tau) \leq M_{2}(\omega) \int_{\tau}^{t} \eta^{-\gamma-1}\left(\frac{\eta}{\tau}\right)^{1-\gamma / 2} \frac{\eta^{\omega-1}}{\sqrt{\eta-\tau}} \frac{t^{3 / 2} \eta^{3 \omega 3 / 2}}{(t-\eta)^{3 / 2}} \exp \left(-C_{2}(\omega) \frac{t \eta^{2 \omega-1}}{t-\eta}\right) d \eta=I_{2}(t, \tau) .
$$

We represent the function $I_{2}$ as a sum of two terms:

$$
I_{2}(t, \tau)=I_{21}(t, \tau)+I_{22}(t, \tau)
$$

for each of which we will have:

$$
\leq \frac{1}{\sqrt{t-\tau}}\left[C_{1}(\omega)+C_{2}(\omega)(\tau / t)^{\varepsilon} \cdot \ln |\tau / t| \cdot(t / \tau)^{\varepsilon}\right]=\frac{1}{\sqrt{t-\tau}}\left[C_{1}(\omega)+C_{3}(\omega)(t / \tau)^{\varepsilon}\right]
$$


For the second term

$$
\begin{gathered}
I_{22}(t, \tau)=\int_{\frac{t+\tau}{2}}^{t} \frac{t \eta^{\omega-1}}{\sqrt{\eta-\tau}(t-\eta)^{\frac{3}{2}}} \exp \left(-C_{2}(\omega) \frac{t \eta^{2 \omega-1}}{t-\eta}\right) d \eta \leq \\
\leq \frac{C(\omega)}{\sqrt{t-\tau}} \int_{\frac{t+\tau}{2}}^{t} \frac{t \eta^{\omega-1}}{(t-\eta)^{\frac{3}{2}}} \exp \left(-C_{3}(\omega) \frac{t(t+\tau)^{2 \omega-1}}{t-\eta}\right) d \eta \leq \\
\leq \frac{C(\omega)}{\sqrt{t-\tau}} \int_{\frac{t+\tau}{2}}^{t} \frac{t \eta^{\omega-1}}{(t-\eta)^{\frac{3}{2}}} \exp \left(-C_{3}(\omega) \frac{t^{2 \omega}}{t-\eta}\left\{1+\frac{\tau}{t}\right\}^{2 \omega-1}\right) d \eta \leq \\
\leq \frac{C(\omega)}{\sqrt{t-\tau}} \int_{0}^{t} \frac{t^{\omega}}{(t-\eta)^{\frac{3}{2}}} \exp \left(-C_{4}(\omega) \frac{t^{2 \omega}}{t-\eta}\right) d \eta=\frac{C_{5}(\omega)}{\sqrt{t-\tau}} \int_{t^{\frac{2 \omega-1}{2}}}^{\infty} \exp \left\{-z^{2}\right\} d z \leq \frac{C_{5}(\omega)}{\sqrt{t-\tau}} .
\end{gathered}
$$

In these inequalities, the constants $C(\omega), C_{j}(\omega), j=1,2,3,4,5$ are different and depend only on $\omega$. The obtained inequalities imply the required estimate (17). This completes the proof of the Theorem.

Remark 3. From relation (13) it follows that homogeneous equation

$$
\varphi(t)-\int_{0}^{t} K_{\omega}(t, \tau) \mu(\tau) d \tau=0, t \in R_{+},
$$

is equivalent to the nonhomogeneous equation:

$$
\varphi(t)+\int_{0}^{t} \overline{\tilde{K}}(t, \tau) \cdot \varphi(\tau) d \tau=C_{0} \cdot \varphi_{\mathrm{hom}}\left((2 \omega-1) \cdot t^{2 \omega-1}\right) .
$$

Study of the boundary value problem

A solution of the original boundary value problem (1)-(2) have the form:

$$
u(x, t)=\int_{0}^{x} v(\xi, t) d \xi
$$

where $v(x, t)=v_{\text {hom }}(x, t)+v_{\text {part }}(x, t)$, and

$$
\begin{gathered}
v_{\text {hom }}(x, t)=\frac{1}{2 a \sqrt{\pi}} \int_{0}^{t} \frac{1}{(t-\tau)^{1 / 2}}\left[-\exp \left\{-\frac{(x+\gamma(\tau))^{2}}{4 a^{2}(t-\tau)}\right\}+\exp \left\{-\frac{(x-\gamma(\tau))^{2}}{4 a^{2}(t-\tau)}\right\}\right] \cdot \varphi_{0}(\tau) d \tau \\
v_{\text {part }}(x, t)=\frac{1}{2 a \sqrt{\pi}} \int_{0}^{t} \frac{1}{(t-\tau)^{1 / 2}}\left[-\exp \left\{-\frac{(x+\gamma(\tau))^{2}}{4 a^{2}(t-\tau)}\right\}+\exp \left\{-\frac{(x-\gamma(\tau))^{2}}{4 a^{2}(t-\tau)}\right\}\right] \cdot \varphi_{\text {part }}(\tau) d \tau+ \\
+\frac{1}{2 a \sqrt{\pi}} \int_{0}^{t} \int_{0}^{\infty} \frac{1}{(t-\tau)^{1 / 2}}\left[-\exp \left\{-\frac{(x+\xi)^{2}}{4 a^{2}(t-\tau)}\right\}+\exp \left\{-\frac{(x-\xi)^{2}}{4 a^{2}(t-\tau)}\right\}\right] \cdot \tilde{f}(\xi, \tau) d \xi d \tau+ \\
\quad+\frac{1}{2 a \sqrt{\pi}} \int_{0}^{t} \frac{x}{(t-\tau)^{3 / 2}} \exp \left\{-\frac{x^{2}}{4 a^{2}(t-\tau)}\right\} v_{0}(\tau) d \tau
\end{gathered}
$$

where the functions $(\gamma(t))^{\frac{3 / 2-\omega}{\omega}} \cdot \varphi(t)$ and $(\gamma(t))^{\frac{3 / 2-\omega}{\omega}} \cdot \tilde{f}(x, t)$ are bounded and continuous functions on $R_{+}$and $Q$, respectively.

From (18)-(20) we obtain the following estimates:

$$
u_{h o m}(x, t) \leq a \sqrt{\pi}\left\{-2 \operatorname{erf}\left(\frac{x}{2 a \sqrt{2 \omega-1} \cdot[\gamma(t)]^{\frac{2 \omega-1}{2 \omega}}}\right)+\right.
$$

Mathematics series. № 1(101)/2021 


$$
\begin{gathered}
+2 \exp \left\{\frac{(2 \omega-1)[\gamma(t)]^{\frac{2 \omega-1}{\omega}}}{4 a^{2}}\right\} \operatorname{erfc}\left(\frac{\sqrt{2 \omega-1} \cdot[\gamma(t)]^{\frac{2 \omega-1}{2 \omega}}}{2 a}\right)- \\
-\exp \left\{\frac{2 x+(2 \omega-1)[\gamma(t)]^{\frac{2 \omega-1}{\omega}}}{4 a^{2}}\right\} \operatorname{erfc}\left(\frac{x+(2 \omega-1)[\gamma(t)]^{\frac{2 \omega-1}{\omega}}}{2 a \sqrt{2 \omega-1} \cdot[\gamma(t)]^{\frac{2 \omega-1}{2 \omega}}}\right)- \\
\left.-\exp \left\{-\frac{2 x-(2 \omega-1)[\gamma(t)]^{\frac{2 \omega-1}{\omega}}}{4 a^{2}}\right\} \operatorname{erfc}\left(-\frac{x-(2 \omega-1)[\gamma(t)]^{\frac{2 \omega-1}{\omega}}}{2 a \sqrt{2 \omega-1}[\gamma(t)]^{\frac{2 \omega-1}{2 \omega}}}\right)\right\} .
\end{gathered}
$$

For $u_{\text {part }}(x, t)$, we have that

$$
\begin{gathered}
u_{\text {part }}(x, t) \leq a \sqrt{\pi}\left\{-2 \operatorname{erf}\left(\frac{x}{2 a \sqrt{2 \omega-1 \cdot} \cdot[\gamma(t)]^{\frac{2 \omega-1}{2 \omega}}}\right)+\right. \\
+2 \exp \left\{\frac{(2 \omega-1)[\gamma(t)]^{\frac{2 \omega-1}{\omega}}}{4 a^{2}}\right\} \operatorname{erfc}\left(\frac{\sqrt{2 \omega-1} \cdot[\gamma(t)]^{\frac{2 \omega-1}{2 \omega}}}{2 a}\right)- \\
-\exp \left\{\frac{2 x+(2 \omega-1)[\gamma(t)]^{\frac{2 \omega-1}{\omega}}}{4 a^{2}}\right\} \operatorname{erfc}\left(\frac{x+(2 \omega-1)[\gamma(t)]^{\frac{2 \omega-1}{\omega}}}{2 a \sqrt{2 \omega-1} \cdot[\gamma(t)]^{\frac{2 \omega-1}{2 \omega}}}\right)- \\
\left.-\exp \left\{-\frac{2 x-(2 \omega-1)[\gamma(t)]^{\frac{2 \omega-1}{\omega}}}{4 a^{2}}\right\} \operatorname{erfc}\left(-\frac{x-(2 \omega-1)[\gamma(t)]^{\frac{2 \omega-1}{\omega}}}{2 a \sqrt{2 \omega-1}[\gamma(t)]^{\frac{2 \omega-1}{2 \omega}}}\right)\right\}+\frac{1}{\sqrt{\pi}} \operatorname{erf}\left(\frac{\sqrt{2 \omega-1}[\gamma(t)]^{\frac{2 \omega-1}{2 \omega}}}{2 a}\right) .
\end{gathered}
$$

Therefore estimates of these integrals give the statement of the Theorem.

Theorem 5. For any right side $f(t) \in L_{\infty}\left(\mathrm{R}_{+} ;[\gamma(t)]^{\frac{3 / 2-\omega}{\omega}} \exp \left\{\gamma(t) /\left(4 a^{2}\right)\right\}\right)$ and for given functions $f(x, t) \in W_{\infty}^{1,0}\left(G ;[\gamma(t)]^{3 / 2 \omega-1} \exp \left\{[\gamma(t)]^{\frac{2 \omega-1}{\omega}} /\left(4 a^{2}\right)\right\}\right), u_{0}(t) \in L_{\infty}\left(R_{+} ;[\gamma(t)]^{\frac{\omega-3 / 2}{\omega}}\right) ; u_{1}(t) \in L_{\infty}\left(\mathrm{R}_{+}\right.$; $[\gamma(t)]^{\frac{3 / 2-\omega}{\omega}}$ ) boundary value problem $(1)-(2)$ has a general solution $u(x, t) \in L_{\infty}\left(G ;\left(x+[\gamma(t)]^{3 / 2 \omega-1}\right)^{-1}\right)$, which is determined from formula (18)-(20).

\section{Acknowledgments}

Supported by the grant projects AP08956033 (2020 - 2021) and AP08855372 (2020 - 2022) from the Ministry of Science and Education of the Republic of Kazakhstan.

\section{References}

1 Карташов Э.М. Аналитические методы в теории теплопроводности твердых тел / Э.М. Карташов. - М.: Высш. шк., 1985. - 553 с.

2 Карташов Э.М. Аналитические методы решения краевых задач уравнения теплопроводности в области с движущимися границами / Э.М. Карташов, Б.Я. Любов // Изв. АН СССР. Энергетика и транспорт. - 1974. - № 6. - С. 83-111.

3 Карташов Э.М. Аналитические методы решения краевых задач нестационарной теплопроводности в областях с движущимися границами / Э.М. Карташов // Изв. РАН. Энергетика. $-1999 .-$ № 5. - C. 3-34.

4 Карташов Э.М. Аналитические методы решения краевых задач нестационарной теплопроводности в областях с движущимися границами / Э.М. Карташов // Инж.-физ. журн. $-2001 .-74 .-$ № 2. - C. 171-195. DOI: 10.1023/A:1016641613982

5 Аттетков А.В. Температурное поле полупространства с термически тонким покрытием в импульсных режимах теплообмена с окружающей средой / А.В. Аттетков, Р.А. Власов, И.К. Волков // Инж.-физ. журн. - 2001. - 74. - № 3. - C. 647-655. DOI: 10.1023/A:1016756227188

6 Kharin S.N. Mathematical models of phenomena in electrical contacts: Monograph / S.N. Kharin // A.P. Ershov Institute of Informatics system, Siberian Branch of RAS. - 2017. - 193. 
7 Amangaliyeva M.M. On one homogeneous problem for the heat equation in an infinite angular domain / M.M. Amangaliyeva, M.T. Jenaliyev, M.T. Kosmakova, M.I. Ramazanov // Sib. Math. Jour. - 2015. 56. - № 6. - P. 82-995.

8 Jenaliev M.T. On a homogeneous parabolic problem in an infinite angular domain / M.T. Jenaliev, S.A. Iskakov, M.I. Ramazanov // Eurasian journal of mathematical and computer applications. - 2019. - 7. - № 1. - P. 38-52.

9 Шишкин А.Г. Математическое моделирование физических процессов в термоядерной и газоразрядной плазме / А.Г. Шишкин. - М.: Аргамак-медиа, 2015. - 235 с.

10 Намитоков K.K. Математическое моделирование процессов в газоразрядной плазме / K.K. Намитоков, П.Л. Пахомов, С.Н. Харин. - Алма-Ата: Наука, 1988. - 208 с.

11 Карташов Э.М. Проблема теплового удара в области с движущейся границей на основе новых интегральных соотношений / Э.М. Карташов // Изв. РАН. Энергетика. - 1997. - 4. - С. 122-137.

12 Веригин Н.Н. Об одном классе гидромеханических задач для областей с подвижными границами / Н.Н. Веригин // Динамика жидкости со свободными границами. - 1980. - 46. - С. 23-32.

13 Багдасаров Х.С. Тепло- и массоперенос при выращивании монокристаллов направленной кристаллизацией / Х.С. Багдасаров, Л.А. Горяинов. - М.: Физматлит, 2007. - 234 с.

14 Солонников В.А. Одномерная параболическая задача, возникающая при исследовании некоторых задач со свободными границами / В.А. Солонников, А. Фазано // Зап. науч. семин. ПОМИ. - 2000. - 269. - С. 322-338.

15 Тихонов А.Н. Уравнения математической физики: учеб. пос. для ун-тов / А.Н. Тихонов, А.А. Самарский. - 4-е изд., испр. - М.: Наука, 1972. - 735 с.

16 Полянин А.Д. Справочник по интегральным уравнениям / А.Д. Полянин, А.В. Манжиров. - М.: Физматлит, 2003. - 608 с.

17 Jenaliyev M.T. On a Volterra equation of the second kind with 'incompressible' kernel / M.T. Jenaliyev, M.M. Amangaliyeva, M.T. Kosmakova and M.I. Ramazanov // Advances in Difference Equations. 2015. - 71. - P. 14

18 Amangaliyeva M.M. About Dirichlet boundary value problem for the heat equation in the infinite angular domain / M.M. Amangaliyeva, M.T. Jenaliyev, M.T. Kosmakova and M.I. Ramazanov // Boundary Value Problem-. - 2014. - 213. - P. 21.

19 Hardy G.G. Inequalities / G.G. Hardy, J.E. Littlewood, G.M. Polya. - Foreign literature, 1948. - 456 p.

\author{
М. Т. Дженалиев, М.И. Рамазанов, А.О. Танин
}

\title{
Шекарасы $x=\gamma(t)$ заңдылығымен қозғалатын Солонников-Фазан есебінің шешімі туралы
}

\begin{abstract}
Жұмыста бастапқы мезетте жойылатын облыстағы жылуөткізгіштік теңдеу үшін шекаралық есептің шешімі зерттелген. Мұнда, шекарасы уақытқа байланысты $x=\gamma(t)$ заңдылығымен өзгереді. Қарастырылып отырған есеп жалпыланған жылу потенциалдарының көмегімен псевдо-вольтерралық интегралдық теңдеуге келтіріледі. Ал интегралдық оператордың нормасының бірге тең болуы оның ерекшелігі болып табылады. Сонымен қатар, сәйкес біртекті интегралдық теңдеудің нөлдік емес шешімінің болатыны көрсетілген.
\end{abstract}

Кілт сөздер: жылуөткізгіштік теңдеу, жылжымалы шекара, жойылатын облыс, псевдо-вольтерралық интегралдық теңдеу. 
М.Т. Дженалиев, М.И. Рамазанов, А.О. Танин

\title{
К решению задачи Солонникова-Фазано при движении границы по произвольному закону $x=\gamma(t)$
}

\begin{abstract}
В работе исследованы вопросы разрешимости граничной задачи для уравнения теплопроводности в области, которая вырождается в точку в начальный момент времени. При этом изменяющаяся со временем граница движется по произвольному закону $x=\gamma(t)$. С помощью обобщенных тепловых потенциалов исследуемая задача редуцируется к псевдо-вольтерровому интегральному уравнению, особенность которого заключается в том, что норма интегрального оператора равна единице. Показано, что соответствующее однородное интегральное уравнение имеет ненулевое решение.
\end{abstract}

Ключевые слова: уравнение теплопроводности, подвижная граница, вырождающаяся область, псевдовольтерровое интегральное уравнение.

\section{References}

1 Kartashov, E.M. (1985). Analiticheskie metody v teorii teploprovodnosti tverdykh tel [Analytic methods in the theory of thermal conductivity of solids/. Moscow: Vysshaia shkola [in Russian].

2 Kartashov, E.M., \& Liubov B.Ya. (1974). Analiticheskie metody resheniia kraevykh zadach uravneniia teploprovodnosti v oblasti s dvizhushchimisia hranitsami [Analytic methods for solving boundary value problems for the heat equation in a region with moving boundaries]. Izvestiia AN SSSR. Enerhetika $i$ transport - Proceeding of the USSR AS. Power engineering and transport, 6, 83-111 [in Russian].

3 Kartashov, E.M. (1999). Analiticheskie metody resheniia kraevykh zadach nestatsionarnoi teploprovodnosti v oblastiakh s dvizhushchimisia hranitsami [Analytical Methods of Solution of Boundary-Value Problems of Nonstationary Heat Conduction in Region with Moving Boundaries]. Izvestiia RAN. Enerhetika-Proceedings of the RAS. Power engineering, 5, 3-34 [in Russian].

4 Kartashov, E.M. (2001). Analytical methods of solution of boundary-value problems of nonstationary heat conduction in region with moving boundaries. Journal of Engineering Physics and Thermophysics, 74(2), 498-536. DOI: 10.1023/A:1016641613982.

5 Attetkov, A.V., Vlasov, P.A., \& Volkov, I.K. (2001). Temperature field of a half-space with a thermally thin coating in pulse modes of heat exchange with the environment. Journal of Engineering Physics and Thermophysics, 74(3), 647-655. DOI: 10.1023/A:1016756227188.

6 Kharin, S.N. (2017). Mathematical models of phenomena in electrical contacts: Monograph. A.P. Ershov Institute of Informatics system, Siberian Branch of RAS, 193.

7 Amangaliyeva, M.M., Jenaliyev, M.T., Kosmakova, M.T., \& Ramazanov, M.I. (2015). On one homogeneous problem for the heat equation in an infinite angular domain. Sib. Math. Jour., 56(6), 982-995.

8 Jenaliev, M.T., Iskakov, S.A., \& Ramazanov, M.I. (2019). On a homogeneous parabolic problem in an infinite angular domain. Eurasian journal of mathematical and computer applications, 7(1), 38-52.

9 Shishkin, A.G. (2015). Matematicheskoe modelirovanie fizicheskikh protsessov v termoiadernoi i hazorazriadnoi plazme [Mathematical modeling of physical processes in a fuel and gas-fueled process]. Moscow: Arhamak-media [in Russian].

10 Namitokov, K.K., Pakhomov, P.L., \& Kharin, S.N. (1988). Matematicheskoe modelirovanie protsessov v hazorazriadnoi plazme [Mathematical Modeling of Phenomena in Gaseous Plasma Discharge]. Alma-Ata: Nauka [in Russian].

11 Kartashov, E.M. (1997). Problema teplovoho udara v oblasti s dvizhushcheisia hranitsei na osnove novykh intehralnykh sootnoshenii [Heatstroke problem in a region with a moving boundary based on new integral relations]. Izvestiia RAN. Enerhetika - Proceedings of the RAS. Power engineering, 4, 122-137 [in Russian].

12 Verigin, N.N. (1980). Ob odnom klasse hidromekhanicheskikh zadach dlia ispolzovaniia s podvizhnymi hranitsami [On a class of hydromechanical problems for domains with moving boundaries]. Dinamika sploshnoi sredy - Fluid dynamics with free boundaries, 46, 23-32 [in Russian]. 
13 Bagdasarov, Kh.S., \& Goryainov, L.A. (2007). Teplo- i massoperenos pri vyrashchivanii monokristallov napravlennoi kristallizatsiei [Heat and mass transfer during the growth of single crystals by deposited crystallization]. Moscow: FML [in Russian].

14 Solonnikov, V.A., \& Fasano, A. (2000). Odnomernaia parabolicheskaia zadacha, voznikaiushchaia pri issledovanii nekotorykh zadach so svobodnymi hranitsami [One-dimensional parabolic problem arising in the studyof some free boundary problems]. Zapiski nauchnykh seminarov POMI - POMI Scientific Workshop Notes 269, 322-338 [in Russian].

15 Tikhonov, A.N., \& Samarskii, A.A. (1972). Uravneniia matematicheskoi fiziki [Equations of mathematical physics]. Moscow: Nauka [in Russian].

16 Polyanin, A.D., \& Manzhirov, A.V. (2003). Spravochnik po intehralnym uravneniiam /Handbook of integral equations]. Moscow: FML [in Russian].

17 Jenaliyev, M.T., Amangaliyeva, M.M., Kosmakova, M.T., \& Ramazanov, M.I. (2015). On a Volterra equation of the second kind with "incompressible"kernel. Advances in DiferenceEquations, 71, 14.

18 Amangaliyeva, M.M., Jenaliyev, M.T., Kosmakova, M.T. \& Ramazanov, M.I. (2014). About Dirichlet boundary value problem for the heat equation in the innite angular domain. Boundary Value Problem, 213, 21.

19 Hardy, G.G, Littlewood, J.E., \& Polya, G. (1948). Inequalities. Moscow: Foreign literature. 


\author{
K.H.F. Jwamer*, Sh.Sh. Ahmed, D.Kh. Abdullah
}

University of Sulaimani, Sulaimani, Kurdistan Region, Iraq

(E-mail: karwan.jwamer@univsul.edu.iq,Shazad.ahmed@univsul.edu.iq,Diar.khalid85@gmail.com)

\title{
Approximate Solution of Volterra Integro-Fractional Differential Equations Using Quadratic Spline Function
}

In this paper, we suggest two new methods for approximating the solution to the Volterra integro-fractional differential equation (VIFDEs), based on the normal quadratic spline function and the second method used the Richardson Extrapolation technique the usage of discrete collocation points. The fractional derivatives are regarded in the Caputo perception. A new theorem for the Richardson Extrapolation points for using the finite difference approximation of Caputo derivative is introduced with their proof. New techniques using the first derivative at the initial point such that obtained by follow two cases the first using trapezoidal rule and the second using the first step of linear spline function using the Richardson Extrapolation method. Specifically, the program is given in examples analysis in Matlab (R2018b). Numerical examples are available to illuminate the productivity and trustworthiness of the methods, as well as, follow the Clenshaw Curtis rule for calculating the required integrals for those equations.

Keywords: Integro-fractional differential equation, Caputo derivative, Quadratic spline, Extrapolation method, Clenshaw.

\section{Introduction}

In this research we will improve a proximity based on the quadratic spline to attain the numerical solution of the following Volterra integro- fractional differential equation (VIFDE's) of the second kind of the form:

$$
{ }_{a}^{C} D_{t}^{\alpha_{n}} u(t)+\sum_{i=1}^{n-1} \mathcal{P}_{i}(t)_{a}^{C} D_{t}^{\alpha_{(n-i)}} u(t)+\mathcal{P}_{n}(t) u(t)=f(t)+\sum_{\ell=0}^{m} \lambda_{\ell} \int_{a}^{t} \mathcal{K}_{\ell}(t, s){ }_{a}^{C} D_{t}^{\beta_{m-\ell}} u(s) d s, \quad t \in[a, b]
$$

Subject to

$$
[u(t)]_{(t=a)}=u_{a}
$$

where $\alpha_{n}>\alpha_{n-1}>\cdots>\alpha_{1}>\alpha_{0}=0$ and $\beta_{m}>\beta_{m-1}>\cdots>\beta_{1}>\beta_{0}=0$

$$
0<\alpha_{i}, \beta_{j} \leq 1
$$

Connected with $N$-condition; $N=\max \left\{n_{i}, m_{i}\right.$ for all $i$ and $\left.j\right\}$, where $\alpha_{i}, \beta_{j} \in \mathbb{R}^{+}, n_{i}-1<\alpha_{i} \leq n_{i}$ and $m_{j}-1<\beta_{j} \leq m_{j}, n_{i}=\left\lceil\alpha_{i}\right\rceil$ and $m_{j}=\left\lceil\beta_{j}\right\rceil$ for all $i=1,2, \ldots, n$ and $j=1,2, \ldots, m, u(t)$ is the unknown function and $\mathcal{K}: S \times \mathbb{R} \rightarrow \mathbb{R}$ (with $S=\{(t, s): a \leq s \leq t \leq b\}$ ) denote a given functions, $f(t), \mathcal{P}_{i}(t)$; $(i=0,1,2, \ldots, n)$ are given continuous real valued function on $I$, and $\lambda_{l}$ is a scalar parameter.

In Eqn.(1), ${ }_{a}^{C} D_{t}^{\alpha}$ and ${ }_{a}^{C} D_{t}^{\beta}$ denotes fractional differential operator where $n-1<\alpha, \beta<n \in N$ in the sense of Caputo and is given by

$$
{ }_{a}^{C} D_{t}^{\alpha} u(t)= \begin{cases}\frac{1}{\Gamma(n-\alpha)} \int_{a}^{t} \frac{u^{(n)}(s)}{(t-s)^{\alpha+1-n}} d s & n-1<\alpha<n \in \mathbb{N} \\ \frac{d^{n}}{d t^{n}} u(t) & \alpha=n \in \mathbb{N}\end{cases}
$$

${ }^{*}$ Corresponding author.

E-mail: karwan.jwamer@univsul.edu.iq 
Properties of the operator ${ }_{a}^{C} D_{t}^{\alpha}$ can be found in [1-5], we mention the following

i. ${ }_{a}^{C} D_{t}^{\alpha}(t-a)^{p}= \begin{cases}0 & \text { if } p \in\{0,1,2, \ldots, n-1\} \\ \frac{\Gamma(p+1)}{\Gamma(p-\alpha+1)}(t-a)^{p-\alpha} & \text { if } p \in \mathbb{N} \text { and } p \geq n \text { or } p \notin \mathbb{N} \text { and } p>n-1\end{cases}$

ii. Let $n-1<\alpha<n, n \in \mathbb{N}$ and $\alpha, A \in \mathbb{R}$ and functions $u(t)=A$ is constant function such that ${ }_{a}^{C} D_{t}^{\alpha} u(t)=0$

iii. If $0<\alpha \leq 1, t_{r} \in \mathbb{R}$ and $m \in \mathbb{R}^{+}$and for any arbitrary $t_{r} \geq a=t_{0}$. Then, for all $a \leq t \leq b[6]$.

$$
{ }_{a}^{C} D_{t}^{\alpha}\left(t-t_{r}\right)^{m}=\left[\sum_{i=0}^{m-1} \frac{(-1)^{i} \Gamma(m+1)(t-a)^{m-a}}{i ! \Gamma(m+1-i-a)}\left(\frac{t_{r}-a}{t-a}\right)^{i}\right]
$$

where $\lceil\alpha\rceil$ denote the smallest integer greater than or equal to $\alpha$. In the present research.

Several methods have been introduced in the literature for the numerical approaches to IFDE's have been recently studied by numerous authors [7-11].

This work is organized as follows: we start by an introduction then focus the fractional differential operator Caputo sense. Preliminaries and discussing numerical methods, quadratic spline function is defined, devoted to applying the integro-fractional differential, and explains some of the theorems that are needed for this work described in section two. Section three the proposed method is applied to two examples. Also a conclusion is given in section five.

\section{Preliminaries}

In this section, we will introduce and study the concepts such that we divided into two subsections.

Definition 1. Clenshaw and Curtis (1960) defined a procedure for evaluating a definite integral by expanding the integrand in the finite Chebyshev series and adding the terms in the series one by one the technique is very effective especially for integral equations [12] as follows.

$$
\int_{-1}^{1} f(x) d x=\sum_{\substack{r=0 \\ r \text { even }}}^{N} \frac{2}{N} \sum_{k=0}^{N "} \cos \left(\frac{r k \pi}{N}\right) f\left(\cos \left(\frac{k \pi}{N}\right)\right) \quad k=0,1, \ldots, N .
$$

Remark 1.

i. The notation $\sum^{\prime \prime}$ means the first and last terms are to be halved before summing.

ii. The transformation $x=\frac{a+b}{2}+\frac{b-a}{2} y$, converting interval $[a, b]$ into $[-1,1]$.

Definition 2. [13] Richardson's extrapolation is used to create high-accuracy result using low-order formulas.

$$
T_{i}^{(m)}=T_{i+1}^{(m-1)}+\frac{T_{i+1}^{(m-1)}-T_{i}^{(m-1)}}{\left(\frac{h_{i}}{h_{i+m}}\right)-1} .
$$

$i=1,2, \ldots, m$ and $m=1,2, \ldots, k-1$. Where $T_{i}^{(m)}$ is an approximate value. Richardson extrapolation using step sizes of $h_{i}, h_{i+1}, \ldots, h_{i+m}$, and $0<m \leq k-1$.

Remark 2. The sequence $\left\{h_{i}\right\}$ usually is of the form

$$
\left\{h_{0}, \frac{h_{0}}{2}, \frac{h_{0}}{4}, \frac{h_{0}}{8}, \frac{h_{0}}{16}, \ldots\right\},\left\{h_{0}, \frac{h_{0}}{2}, \frac{h_{0}}{3}, \frac{h_{0}}{4}, \frac{h_{0}}{5}, \ldots\right\} \text { and }\left\{h_{0}, \frac{h_{0}}{2}, \frac{h_{0}}{3}, \frac{h_{0}}{4}, \frac{h_{0}}{6}, \frac{h_{0}}{8}, \frac{h_{0}}{12}, \ldots\right\}
$$

In this paper we will consider the sequence $\left\{h_{i}\right\}$ as the form $\left\{h_{0}, \frac{h_{0}}{2}, \frac{h_{0}}{4}, \frac{h_{0}}{8}, \frac{h_{0}}{16}, \ldots\right\}$. 


\subsection{Quadratic Classic Spline $Q(t):[14]$}

A function $Q(t)$ is a piecewise spline of degree two can be written as:

$$
Q(t)= \begin{cases}Q_{0}(t) & t \in\left[t_{0}, t_{1}\right] \\ Q_{1}(t) & t \in\left[t_{1}, t_{2}\right] \\ \vdots & \vdots \\ Q_{N-1}(t) & t \in\left[t_{N-1}, t_{N}\right]\end{cases}
$$

A quadratic spline consisting of $N$ separate pieces of quadratic functions of the form

$$
Q_{r}(t)=a_{r} t^{2}+b_{r} t+c_{r}, t \in\left[t_{r}, t_{r+1}\right], \forall r=0,1, \ldots, N-1 .
$$

In addition $Q(t)$ satisfy the following conditions:

1 The domain of $Q$ is an interval $[a, b]$.

$2 Q$ and $Q^{\prime}$ are continuous on $[a, b]$.

3 There are points $t_{r}$ such that $a=t_{0}<t_{1}<\cdots<t_{N}=b$ and $Q$ is a polynomial of degree two on each subinterval $\left[t_{r}, t_{r+1}\right]$.

4 A quadratic spline is a continuously differentiable piecewise quadratic function.

5 A quadratic spline is a linear combination of basic functions $1, t, t^{2}$. The smoothness condition is stronger than that for the first-degree spline.

6 The interpolating condition $Q_{r}\left(t_{r}\right)=Q_{r}$ and $Q_{r}\left(t_{r+1}\right)=Q_{r+1}$.

We drive the equation for interpolating quadratic spline $Q(t)$, after some manipulation we obtain,

$$
Q(t)=\left(1-\left(\frac{t-t_{r}}{h}\right)^{2}\right) Q_{r}+\left(\frac{t-t_{r}}{h}\right)^{2} Q_{r+1}+\frac{\left(t-t_{r}\right)\left(t_{r+1}-t\right)}{h} Q_{r}^{\prime},
$$

Where $Q_{r}\left(t_{r}\right)=Q_{r}, Q_{r+1}=Q_{r}\left(t_{r+1}\right), Q_{r}^{\prime}\left(t_{r}\right)=m_{r}, Q_{r}^{\prime}=Q_{r}^{\prime}\left(t_{r}\right)$ and $t_{r+1}-t_{r}=h$ for $r=0,1, \ldots, N-1$. We require from the continuously differentiable condition of quadratic spline function i.e. $Q_{r}^{\prime}\left(t_{r+1}\right)=Q_{r+1}^{\prime}$, differentiating Eqn.(3) with respect to $t$, we get

$$
Q_{r}^{\prime}(t)=-\frac{2\left(t-t_{r}\right)}{h^{2}} Q_{r}\left(t_{r}\right)+\frac{2\left(t-t_{r}\right)}{h^{2}} Q_{r}\left(t_{r+1}\right)+\frac{\left(t_{r+1}-t\right)-\left(t-t_{r}\right)}{h} Q_{r}^{\prime}\left(t_{r}\right)
$$

Putting $t=t_{r+1}$, we obtain $Q_{r}^{\prime}\left(t_{r+1}\right)=-Q_{r}^{\prime}\left(t_{r}\right)+2\left(\frac{Q_{r}\left(t_{r+1}\right)-Q_{r}\left(t_{r}\right)}{h}\right)$

$$
Q_{r+1}^{\prime}=-Q_{r}^{\prime}+2\left(\frac{Q_{r+1}-Q_{r}}{h}\right), r=1,2, \ldots, N .
$$

\subsection{Fractional Derivative of Spline Functions}

In this section, we discuss the way of obtaining fractional derivative for all the quadratic spline. So that, here we apply Caputo properties to accomplish.

Lemma 1. The fractional derivative of quadratic spline of order $\alpha$ with respect to $t$ as:

$$
\begin{aligned}
{ }_{a}^{C} D_{t}^{\alpha} Q(t) & =\frac{(t-a)^{1-\alpha}}{h \Gamma(3-a)}\left[\frac{-1}{h}\left(2(t-a)-2(2-\alpha)\left(t_{r}-a\right)\right) Q_{r}+\frac{1}{h}\left(2(t-a)-2(2-\alpha)\left(t_{r}-a\right)\right) Q_{r+1}\right. \\
& \left.+\left[\left(t_{r+1}+t_{r}-2 a\right)(2-\alpha)-2(t-a)\right] Q_{r}^{\prime}\right], \quad \text { where } 0<\alpha \leq 1 .
\end{aligned}
$$

Proof. Quadratic spline function $\mathrm{Q}(\mathrm{t})$ in the interval $\left[t_{r}, t_{r+1}\right]$ give the formula in Eqn.(3)

$$
Q(t)=A_{r}(t) Q_{r}+B_{r}(t) Q_{r+1}+B_{r}(t) Q_{r}^{\prime},
$$

where:

$$
A_{r}(t)=1-\left(\frac{t-t_{r}}{h}\right)^{2}, B_{r}(t)=1-A_{r}(t)=\left(\frac{t-t_{r}}{h}\right)^{2}
$$




$$
\begin{gathered}
C_{r}(t)=\frac{\left(t-t_{r}\right)\left(t_{r+1}-t\right)}{h}, \forall r=0,1, \ldots, N-1 . \\
{ }_{a}^{C} D_{t}^{\alpha} Q(t)=Q_{r a}^{C} D_{t}^{\alpha}\left[1-\left(\frac{t-t_{r}}{h}\right)^{2}\right]+Q_{r+1}{ }_{a}^{C} D_{t}^{\alpha}\left(\frac{t-t_{r}}{h}\right)^{2}+Q_{r a}^{\prime} D_{t}^{\alpha} \frac{\left(t-t_{r}\right)\left(t_{r+1}-t\right)}{h}
\end{gathered}
$$

Using the definition of the Caputo fractional derivative in the from (1)-(3), we have

$$
\begin{aligned}
{ }_{a}^{C} D_{t}^{\alpha} Q(t) & =\frac{-1}{h^{2}}\left[\frac{2(t-a)^{2-\alpha}}{\Gamma(3-\alpha)}-\frac{2(t-a)^{1-\alpha}}{\Gamma(2-\alpha)}\left(t_{r}-a\right)\right] Q_{r}+\frac{1}{h^{2}}\left[\frac{2(t-a)^{2-\alpha}}{\Gamma(3-\alpha)}-\frac{2(t-a)^{1-\alpha}}{\Gamma(2-\alpha)}\left(t_{r}-a\right)\right] Q_{r+1} \\
& +\frac{1}{h}\left[\left(t_{r+1}+t_{r}-2 a\right) \frac{(t-a)^{1-\alpha}}{\Gamma(2-\alpha)}-\frac{2(t-a)^{2-\alpha}}{\Gamma(3-\alpha)}\right] Q_{r}^{\prime}
\end{aligned}
$$

Through calculation, you can get

$$
\begin{gathered}
{ }_{a}^{C} D_{t}^{\alpha} Q(t)=\frac{(t-a)^{1-\alpha}}{h \Gamma(3-a)}\left[\frac{-1}{h}\left(2(t-a)-2(2-\alpha)\left(t_{r}-a\right)\right) Q_{r}+\frac{1}{h}\left(2(t-a)-2(2-\alpha)\left(t_{r}-a\right)\right) Q_{r+1}\right. \\
\left.+\left[\left(t_{r+1}+t_{r}-2 a\right)(2-\alpha)-2(t-a)\right] Q_{r}^{\prime}\right], \quad \text { where } \quad 0<\alpha \leq 1 .
\end{gathered}
$$

Theorem 1. [6] The finite difference approximation of Caputo derivative for $0<\alpha \leq 1$ at define points $t=t_{r+1} ; r=0,1, \ldots, N-1$ and $h=(b-a) / N$, is formed as

$$
{ }_{a}^{C} D_{t}^{\alpha} u\left(t_{r+1}\right)=\frac{h^{-\alpha}}{\Gamma(2-\alpha)} \sum_{j=0}^{r}\left[u\left(t_{r-j+1}\right)-u\left(t_{r-j}\right)\right] b_{j}^{\alpha}
$$

where $b_{j}^{\alpha}=(j+1)^{1-\alpha}-j^{1-\alpha}$.

Theorem 2. (new) The finite difference approximation of Caputo derivative for $0<\alpha \leq 1$ of the subinterval $\left[t_{r}, t_{r+1}\right]$, define the point $t=t_{r}+(i+1) h_{M^{*}}, h_{M^{*}}=\frac{h}{2^{M^{*}}}, h=(b-a) / N$, according the step size of Richardson Extrapolation $M^{*}=0,1, \ldots, M$, is formed as

$$
\begin{aligned}
\left.{ }_{a}^{C} D_{t}^{\alpha} u(t)\right|_{t=t_{r}+(i+1) h_{M^{*}}} & =\frac{h^{-\alpha}}{\Gamma(2-\alpha)} \sum_{j=0}^{r-1}\left[u\left(t_{r-j}\right)-u\left(t_{r-j-1}\right)\right] C_{i, j}^{M^{*}, \alpha} \\
& +\frac{h_{M^{*}}^{-\alpha}}{\Gamma(2-\alpha)} \sum_{j=0}^{i}\left[u\left(t_{r}+(i-j+1) h_{M^{*}}\right)-u\left(t_{r}+(i-j) h_{M^{*}}\right)\right] b_{j}^{\alpha},
\end{aligned}
$$

where $b_{j}^{\alpha}=(j+1)^{1-\alpha}-j^{1-\alpha}, C_{i, j}^{M^{*}, \alpha}=\left[\left((j+1)+\frac{i+1}{2^{M^{*}}}\right)^{1-\alpha}-\left(j+\frac{(i+1)}{2^{M^{*}}} h_{M^{*}}\right)^{1-\alpha}\right] \cdot r=0,1, \ldots, N-1$, $i=0,1, \ldots, 2^{M^{*}}-1, h=t_{r+1}-t_{r}$.

Proof. Recall the definition of Caputo fractional derivative for $0<\alpha \leq 1$ and using first order forward difference approximation [15], to obtain

$$
\left.{ }_{a}^{C} D_{t}^{\alpha} u(t)\right|_{t=t_{r}+(i+1) h_{M^{*}}}=\frac{1}{\Gamma(1-\alpha)} \int_{a}^{t_{r}+(i+1) h_{M^{*}}} \frac{\partial u(s) / \partial s}{\left(t_{r}+(i+1) h_{M^{*}}-s\right)^{\alpha}} d s,
$$




$$
\begin{aligned}
\left.{ }_{a}^{C} D_{t}^{\alpha} u(t)\right|_{t=t_{r}+(i+1) h_{M^{*}}} & =\frac{1}{\Gamma(1-\alpha)}\left\{\int_{a}^{t_{r}} \frac{\partial u(s) / \partial s}{\left(t_{r}+(i+1) h_{M^{*}}-s\right)^{\alpha}} d s+\int_{t_{r}}^{t_{r}+(i+1) h_{M^{*}}} \frac{\partial u(s) / \partial s}{\left(t_{r}+(i+1) h_{M^{*}}-s\right)^{\alpha}} d s\right\} \\
& =\frac{1}{\Gamma(1-\alpha)}\left\{\sum_{\ell=0}^{r-1} \int_{a+\ell h}^{a+(\ell+1) h} \frac{\partial u(s) / \partial s}{\left(t_{r}+(i+1) h_{M^{*}}-s\right)^{\alpha}} d s\right. \\
& \left.+\sum_{\ell=0}^{i} \int_{t_{r}+\ell h_{M^{*}}}^{t_{r}+(\ell+1) h_{M^{*}}} \frac{\partial u(s) / \partial s}{\left(t_{r}+(i+1) h_{M^{*}}-s\right)^{\alpha}} d s\right\} \\
& =\frac{1}{\Gamma(1-\alpha)}\left\{\sum_{\ell=0}^{r-1} \frac{u\left(t_{\ell+1}\right)-u\left(t_{\ell}\right)}{h} \int_{a+\ell h}^{a+(\ell+1) h} \frac{d s}{\left(t_{r}+(i+1) h_{M^{*}}-s\right)^{\alpha}}\right. \\
& \left.+\sum_{\ell=0}^{i} \frac{u\left(t_{r}+(\ell+1) h_{M^{*}}\right)-u\left(t_{r}+\ell h_{M^{*}}\right)}{h_{M^{*}}} \int_{t_{r}+\ell h_{M^{*}}}^{t_{r}+(\ell+1) h_{M^{*}}} \frac{d s}{\left(t_{r}+(i+1) h_{M^{*}}-s\right)^{\alpha}}\right\} .
\end{aligned}
$$

By assumption Let $\xi=t_{r}+(i+1) h_{M^{*}}-s$ then $d \xi=-d s$, if $s=a+\ell h$ then $\xi=(r-\ell) h+(i+1) h_{M^{*}}$ $s=a+(\ell+1) h$ then $\xi=(r-\ell-1) h+(i+1) h_{M^{*}}$, if $s=t_{r}+\ell h_{M^{*}}$ then $\xi=(i-\ell+1) h_{M^{*}} s=t_{r}+(\ell+1) h_{M^{*}}$ then $\xi=(i-\ell) h_{M^{*}}$, we obtain

$$
\begin{aligned}
\left.{ }_{a}^{C} D_{t}^{\alpha} u(t)\right|_{t=t_{r}+(i+1) h_{M^{*}}} & =\frac{1}{\Gamma(1-\alpha)}\left\{\sum_{\ell=0}^{r-1} \frac{u\left(t_{\ell+1}\right)-u\left(t_{\ell}\right)}{h} \int_{(r-\ell-1) h+(i+1) h_{M^{*}}}^{(r-\ell) h+(i+1) h_{M^{*}}} \frac{d \xi}{\xi^{\alpha}}\right. \\
& \left.+\sum_{\ell=0}^{i} \frac{u\left(t_{r}+(\ell+1) h_{M^{*}}\right)-u\left(t_{r}+\ell h_{M^{*}}\right)}{h_{M^{*}}} \int_{(i-\ell) h_{M^{*}}}^{(i-\ell+1) h_{M^{*}}} \frac{d \xi}{\xi^{\alpha}}\right\} .
\end{aligned}
$$

For first sum; let $j=r-\ell-1$ and the second let $j=i-\ell$

$$
\begin{aligned}
& =\frac{1}{\Gamma(1-\alpha)}\left\{\sum_{\ell=0}^{r-1} \frac{u\left(t_{r-j}\right)-u\left(t_{r}-j-1\right)}{h} \int_{j h+(i+1) h_{M^{*}}}^{(j+1) h+(i+1) h_{M^{*}}} \frac{d \xi}{\xi^{\alpha}}\right. \\
& \left.+\sum_{j=0}^{i} \frac{u\left(t_{r}+(i-j+1) h_{M^{*}}\right)-u\left(t_{r}+(i-j) h_{M^{*}}\right)}{h_{M^{*}}} \int_{j h_{M^{*}}}^{(i+1) h_{M^{*}}} \frac{d \xi}{\xi^{\alpha}}\right\} .
\end{aligned}
$$

After integrating then compute we can obtain,

$$
\begin{gathered}
\left.{ }_{a}^{C} D_{t}^{\alpha} u(t)\right|_{t=t_{r}+(i+1) h_{M^{*}}}=\frac{h^{-\alpha}}{\Gamma(2-\alpha)} \sum_{j=0}^{r-1}\left[u\left(t_{r-j}\right)-u\left(t_{r-j-1}\right)\right] C_{i, j}^{M^{*}, \alpha} \\
+\frac{h_{M^{*}}^{-\alpha}}{\Gamma(2-\alpha)} \sum_{j=0}^{i}\left[u\left(t_{r}+(i-j+1) h_{M^{*}}\right)-u\left(t_{r}+(i-j) h_{M^{*}}\right) b_{j}^{\alpha}\right]
\end{gathered}
$$

where $C_{i, j}^{M^{*}, \alpha}=\left[\left((j+1)+\frac{(i+1)}{2^{M^{*}}}\right)^{1-\alpha}-\left(j+\frac{(i+1)}{2^{M^{*}}} h_{M^{*}}\right)^{1-\alpha}\right], \quad b_{j}^{\alpha}=\left[(j+1)^{1-\alpha}+j^{1-\alpha}\right]$

\section{Methods Analysis}

To find numerical solution of Eqn.(1), using quadratic spline function, we can use the following two cases. The first one using the normal and the second using extrapolation method. Now we can drive each cases.

\subsection{Normal Quadratic spline function (VFID's)}

To progress the quadratic spline approximation method for solving Volterra integro-fractional differential equation from Eqns.(1) and (2) on the interval $[a, b]$. First divided the interval $[a, b]$ be into $N$-equal subintervals of length of $h=\frac{b-a}{N}$ with endpoints, the quadratic spline $Q(t)$ interpolating the function $u(t)$ at the grid points are specified by the equation.

$$
{ }_{a}^{C} D_{t}^{\alpha_{n}} Q(t)+\sum_{i=1}^{n-1} \mathcal{P}_{i}(t)_{a}^{C} D_{t}^{\alpha_{(n-i)}} Q(t)+\mathcal{P}_{n}(t) Q(t)=f(t)+\sum_{\ell=0}^{m} \lambda_{\ell} \int_{a}^{t} \mathcal{K}_{\ell}(t, s){ }_{a}^{C} D_{t}^{\beta_{m-\ell}} Q(s) d s .
$$


Substituting $t=t_{r+1}, r=0,1,2, \ldots, N-1$, from Eqn.(5) then collocate Eqn.(5) at the uniform grid points after substituting Eqn.(4) into Eqn.(5) then we obtain

$$
\begin{gathered}
\frac{((r+1) h)^{1-\alpha_{n}}}{\Gamma\left(3-\alpha_{n}\right)}\left\{\frac{2}{h}\left[(1-r) \alpha_{n} r\right]\left(Q_{r+1}-Q_{r}\right)-\left[\alpha_{n}(2 r+1)-2 r\right] Q_{r}^{\prime}\right\} \\
+\sum_{i=1}^{n-1} p_{i}\left(t_{r+1}\right) \frac{((r+1) h)^{1-\alpha_{n}-i}}{\Gamma\left(3-\alpha_{n-i}\right)}\left\{\frac{2}{h}\left[(1-r) \alpha_{n-i} r\right]\left(Q_{r+1}-Q_{r}\right)-\left[\alpha_{n-i}(2 r+1)-2 r\right] Q_{r}^{\prime}\right\}+p_{n(r+1)} Q_{r+1} \\
=f_{r+1}+\sum_{\ell=0}^{m-1} \lambda_{\ell}\left\{\sum_{j=0}^{r-1} \int_{t_{j}}^{t_{j+1}} \mathcal{K}_{\ell}\left(t_{r+1}, s\right)\left[Q_{j a}^{C} D_{s}^{\beta_{m-\ell}} A_{j}(s)+Q_{j+1}{ }_{a}^{C} D_{s}^{\beta_{m-\ell}} B_{j}(s)+Q_{j a}^{\prime}{ }_{j}^{C} D_{s}^{\beta_{m-\ell}} C_{j}(s)\right] d s\right. \\
\left.+\int_{t_{r}}^{t_{r+1}} \mathcal{K}_{\ell}\left(t_{r+1}, s\right)\left[Q_{r}{ }_{a}^{C} D_{s}^{\beta_{m-\ell}} A_{r}(s)+Q_{r+1}{ }_{a}^{C} D_{s}^{\beta_{m-\ell}} B_{r}(s)+Q_{r}^{\prime}{ }_{a}^{C} D_{s}^{\beta_{m-\ell}} C_{r+1}(s)\right] d s\right\} \\
+\lambda_{m}\left\{\sum_{j=0}^{r-1} \int_{t_{j}}^{t_{j+1}} \mathcal{K}_{m}\left(t_{r+1}, s\right)\left[A_{j}(s) Q_{j}+B_{j}(s) Q_{j+1}+C_{j}(s) Q_{j}^{\prime}\right] d s\right. \\
\left.+\int_{t_{r}}^{t_{r+1}} \mathcal{K}_{m}\left(t_{r+1}, s\right)\left[A_{r}(s) Q_{r}+B_{r}(s) Q_{r+1}+C_{r}(s) Q_{r}^{\prime}\right] d s\right\} .
\end{gathered}
$$

Let $\mathcal{W}_{n}^{r}(s)=P_{s(r+1)} \frac{((r+1) h)^{1-\alpha_{n-s}}}{\Gamma\left(3-\alpha_{n-s}\right)} \frac{2}{h}\left((1-r)+\alpha_{(n-s) r}\right), \mathcal{H}_{n}^{r}=P_{n(r+1)}+\sum_{s=0}^{n-1} \mathcal{W}_{n}^{r}(s)$ and

$$
\mathcal{V}_{n}^{r}(s)=P_{s(r+1)} \frac{((r+1) h)^{1-\alpha_{n-s}}}{\Gamma\left(3-\alpha_{n-s}\right)}\left(\alpha_{(n-s)}(2 r+1)-2 r\right), s=0,1, \ldots, n-1, r=0,1,2, \ldots, N-1
$$

The Eqn.(6) becomes,

$$
\begin{gathered}
Q_{r+1}\left\{\mathcal{H}_{n}^{r}-\lambda_{m} \int_{t_{r}}^{t_{r+1}} \mathcal{K}_{m}\left(t_{r+1}, s\right) B_{r}(s) d s-\sum_{\ell=0}^{m-1} \lambda_{\ell} \int_{t_{r}}^{t_{r+1}} \mathcal{K}_{\ell}\left(t_{r+1}, s\right)_{a}^{C} D_{s}^{\beta_{m-\ell}} B_{r}(s) d s\right\} \\
=Q_{r}\left\{\sum_{s=0}^{n-1} \mathcal{W}_{n}^{r}(s)+\sum_{\ell=0}^{m-1} \lambda_{\ell} \int_{t_{r}}^{t_{r+1}} \mathcal{K}_{\ell}\left(t_{r+1}, s\right)_{a}^{C} D_{s}^{\beta_{m-\ell}} A_{r}(s) d s+\lambda_{m} \int_{t_{r}}^{t_{r+1}} \mathcal{K}_{m}\left(t_{r+1}, s\right) A_{r}(s) d s\right\} \\
+Q_{r}^{\prime}\left\{\sum_{s=0}^{n-1} \mathcal{V}_{n}^{r}(s)+\sum_{\ell=0}^{m-1} \lambda_{\ell} \int_{t_{r}}^{t_{r+1}} \mathcal{K}_{\ell}\left(t_{r+1}, s\right)_{a}^{C} D_{s}^{\beta_{m-\ell}} C_{r+1}(s) d s+\lambda_{m} \int_{t_{r}}^{t_{r+1}} \mathcal{K}_{m}\left(t_{r+1}, s\right) C_{r+1}(s) d s\right\}+f_{r+1} \\
+\sum_{\ell=0}^{m-1} \lambda_{\ell}\left\{\sum_{j=0}^{r-1} \int_{t_{j}}^{t_{j+1}} \mathcal{K}_{\ell}\left(t_{r+1}, s\right)\left[Q_{j a}^{C} D_{s}^{\beta_{m-\ell}} A_{j}(s)+Q_{j+1}^{C} D_{s}^{\beta_{m-\ell}} B_{j}(s)+Q_{j a}^{\prime} D_{s}^{\beta_{m-\ell}} C_{j}(s)\right] d s\right\} \\
+\lambda_{m} \sum_{j=0}^{r-1} \int_{t_{j}}^{t_{j+1}} \mathcal{K}_{m}\left(t_{r+1}, s\right)\left[A_{j}(s) Q_{j}+B_{j}(s) Q_{j+1}+C_{j}(s) Q_{j}^{\prime}\right] d s .
\end{gathered}
$$

To acquire $Q_{1}$, inserting $r=0$ into Eqn.(7), we must enter $Q_{0}$ and $Q_{0}^{\prime}$ into Eqn.(7) but as we see we have the best $Q_{0}$ from the initial condition. To getting $Q_{0}^{\prime}$ from the forward formula, we ought to have $Q_{1}^{(p)}$. So for preparing $Q_{1}^{(p)}$ we've critical approaches: (I) Trapezoidal Method and (II) First step of the linear spline.

I- Using Trapezoidal Method

Calculate the Eqn.(1)on interval $\left[t_{0}, t_{1}\right]$ we obtain,

$$
\begin{gathered}
\left.{ }_{a}^{C} D_{t}^{\alpha_{n}} u(t)\right|_{t=t_{1}}+\left.\sum_{i=1}^{n-1} p_{i}\left(t_{1}\right)_{a}^{C} D_{t}^{\alpha_{n-i}} u(t)\right|_{t=t_{1}}+p_{n}\left(t_{1}\right) u_{1} \\
=f\left(t_{1}\right)+\sum_{\ell=0}^{m-1} \lambda_{\ell} \int_{a}^{t_{1}} \mathcal{K}_{\ell}\left(t_{1}, s\right)_{a}^{C} D_{s}^{\beta_{m-\ell}} u(s) d s+\lambda_{m} \int_{a}^{t_{1}} \mathcal{K}_{m}\left(t_{1}, s\right) u(s) d s
\end{gathered}
$$

Recall the Theorem (1)

$$
\left.{ }_{a}^{C} D_{t}^{\alpha} u(t)\right|_{t=t_{i}}=\frac{h^{-\alpha}}{\Gamma(2-\alpha)} \sum_{j=0}^{i-1}\left[u\left(t_{i-j}\right)-u\left(t_{i-j-1}\right)\right] b_{j}^{\alpha}
$$


where

$$
b_{j}^{\alpha}=(j+1)^{1-\alpha}-j^{1-\alpha} .
$$

First we can find $\left.{ }_{a}^{C} D_{t}^{\alpha} u(t)\right|_{t=t_{1}}=\frac{h^{-\alpha}}{\Gamma(2-\alpha)}\left[u_{1}-u_{0}\right], \int_{a}^{t_{1}} \mathcal{K}_{\ell}\left(t_{1}, s\right)_{a}^{C} D_{s}^{\beta_{m-\ell}} u(s) d s=\frac{h^{1-\beta_{m-\ell}}}{2 \Gamma\left(2-\beta_{m-\ell}\right)} \mathcal{K}_{11}^{\ell}\left[u_{1}-u_{0}\right]$ and $\int_{a}^{t_{1}} \mathcal{K}_{m}\left(t_{1}, s\right) u(s) d s=\frac{h}{2}\left[\mathcal{K}_{10}^{m} u_{0}+\mathcal{K}_{11}^{m} u_{1}\right]$, the Eqn.(8) becomes

$$
\begin{gathered}
\frac{h^{-\alpha_{n}}}{\Gamma\left(2-\alpha_{n}\right)}\left[u_{1}-u_{0}\right]+\sum_{i=1}^{n-1} p_{i}\left(t_{1}\right) \frac{h^{-\alpha_{n}-i}}{\Gamma\left(2-\alpha_{n}-i\right)}\left[u_{1}-u_{0}\right]+p_{n}\left(t_{1}\right) u_{1} \\
=f\left(t_{1}\right)+\sum_{\ell=0}^{m-1} \lambda_{\ell} \frac{h^{1-\beta_{m-\ell}}}{2 \Gamma\left(2-\beta_{m-\ell}\right)} \mathcal{K}_{11}^{\ell}\left[u_{1}-u_{0}\right]+\lambda_{m} \frac{h}{2}\left[\mathcal{K}_{10}^{m} u_{0}+\mathcal{K}_{11}^{m} u_{1}\right] \\
u_{1}\left\{\frac{h^{-\alpha_{n}}}{\Gamma\left(2-\alpha_{n}\right)}+\sum_{i=1}^{n-1} p_{i}\left(t_{1}\right) \frac{h^{-\alpha_{n}-i}}{\Gamma\left(2-\alpha_{n}-i\right)}+p_{n}\left(t_{1}\right)-\sum_{\ell=0}^{m-1} \lambda_{\ell} \frac{h^{1-\beta_{m-\ell}}}{2 \Gamma\left(2-\beta_{m-\ell}\right)} \mathcal{K}_{11}^{\ell}-\lambda_{m} \frac{h}{2} \mathcal{K}_{11}^{m}\right\} \\
=u_{0}\left\{\frac{h^{-\alpha_{n}}}{\Gamma\left(2-\alpha_{n}\right)}+\sum_{i=1}^{n-1} p_{i}\left(t_{1}\right) \frac{h^{-\alpha_{n}-i}}{\Gamma\left(2-\alpha_{n}-i\right)}-\sum_{\ell=0}^{m-1} \lambda_{\ell} \frac{h^{1-\beta_{m-\ell}}}{2 \Gamma\left(2-\beta_{m-\ell}\right)} \mathcal{K}_{11}^{\ell}+\lambda_{m} \frac{h}{2} \mathcal{K}_{10}^{m}\right\}+f\left(t_{1}\right)
\end{gathered}
$$

So $u_{1} \approx Q_{1}^{(p)}$.

\section{II- First step of linear spline(using R-Extrapolation Technique)}

Calculate the Eqn.(1) on interval $\left[t_{0}, t_{1}\right], t=t_{0}+(i+1) h_{M^{*}}, i=0,1, \ldots, 2^{M^{*}}-1, M^{*}=0,1, \ldots, M$. and $h_{M^{*}}=\frac{h}{2^{M^{*}}}$, is formed as

$$
\begin{gathered}
\left.{ }_{a}^{C} D_{t}^{\alpha_{n}} u(t)\right|_{t=t_{0}+(i+1) h_{M^{*}}}+\left.\sum_{i=1}^{n-1} P_{i}\left(t_{0}+(i+1) h_{M^{*}}\right)_{a}^{C} D_{t}^{\alpha_{n-i}} u(t)\right|_{t=t_{0}+(i+1) h_{M^{*}}} \\
+\left.p_{n}\left(t_{0}+(i+1) h_{M^{*}}\right) u(t)\right|_{t=t_{0}+(i+1) h_{M^{*}}}=f\left(t_{0}+(i+1) h_{M^{*}}\right) \\
+\sum_{\ell=0}^{m-1} \lambda_{\ell} \int_{a}^{t_{0}+(i+1) h_{M^{*}}} k_{\ell}\left(t_{0}+(i+1) h_{M^{*}}, s\right)_{a}^{C} D_{s}^{\beta_{m-\ell}} u(s) d s \\
\quad+\lambda_{m} \int_{a}^{t_{0}+(i+1) h_{M^{*}}} k_{m}\left(t_{0}+(i+1) h_{M^{*}}, s\right) u(s) d s
\end{gathered}
$$

Since by finite difference approximation and recall the theorem (2)

$$
\left.{ }_{a}^{C} D_{t}^{\alpha} u(t)\right|_{t=t_{0}+(i+1) h_{M^{*}}}=\frac{h_{M^{*}}^{-\alpha}}{\Gamma(2-\alpha)} \sum_{j=0}^{i}\left[u\left(t_{r}+(i-j+1) h_{M^{*}}\right)-u\left(t_{r}+(i-j) h_{M^{*}}\right)\right] b_{j}^{\alpha}
$$

if $M^{*}=0, i=0, t=t_{0}+(0+1) h_{0^{*}}$ and $h_{0^{*}}=\frac{h}{2^{0^{*}}}=h$, we obtain $=\frac{h_{0}^{-\alpha}}{\Gamma(2-\alpha)}\left[u_{0,1}^{0}-u_{0,0}^{0}\right] b_{0}^{\alpha}=\frac{h^{-\alpha}}{\Gamma(2-\alpha)}\left[u_{1}-u_{0}\right]$, the equation (10) becomes

$$
\begin{aligned}
& \frac{h_{M^{*}}^{-\alpha_{n}}}{\Gamma\left(2-\alpha_{n}\right)}\left\{\left[u_{M^{*}, i+1}^{0}-u_{M^{*}, i}^{0}\right]+\sum_{j=1}^{i}\left[u_{M^{*}, i-j+1}^{0}-u_{M^{*}, i-j}^{0}\right] b_{j}^{\alpha_{n}}\right\} \\
& \quad+\sum_{i i=1}^{n-1} P_{i i}\left(t_{0}+(i+1) h_{M^{*}}\right) \frac{h_{m^{*}}^{-\alpha_{n-i i}}}{\Gamma\left(2-\alpha_{n-i i}\right)}\left\{\left[u_{M^{*}, i+1}^{0}-u_{M^{*}, i}^{0}\right]\right. \\
& \left.+\sum_{j=1}^{i}\left[u_{M^{*}, i-j+1}^{0}-u_{M^{*}, i-j}^{0}\right] b_{j}^{\alpha_{n-i i}}\right\}+p_{n}\left(t_{0}+(i+1) h_{M^{*}}\right) u_{M^{*}, i+1}^{0}
\end{aligned}
$$




$$
\begin{gathered}
=f\left(t_{0}+(i+1) h_{M^{*}}\right)+\sum_{\ell=0}^{m-1} \lambda_{\ell} \sum_{\ell=0}^{m-1} \lambda_{\ell} \frac{h_{M^{*}}}{2} \\
\left\{2 \sum_{d=0}^{i} k_{\ell}\left(t_{0}+(i+1) h_{M^{*}}, t_{0}+d h_{M^{*}}\right) \frac{h_{M^{*}-\ell}}{\Gamma\left(2-\beta_{m-\ell}\right)} \sum_{j=0}^{d-1}\left[u_{M^{*}, d-j}^{0}-u_{M^{*}, d-j-1}^{0}\right] b_{j}^{\beta_{m-\ell}}\right. \\
+k_{\ell}\left(t_{0}+(i+1) h_{M^{*}}, t_{0}+(i+1) h_{M^{*}}\right) \frac{h_{M^{*}-\ell}^{-\beta_{m}}}{\Gamma\left(2-\beta_{m-\ell}\right)}\left[\left(u_{M^{*}, i+1}^{0}-u_{M^{*}, i}^{0}\right)\right. \\
\left.\left.+\sum_{j=1}^{i}\left(u_{M^{*}, i-j+1}^{0}-u_{M^{*}, i-j}^{0}\right) b_{j}^{\beta_{m-\ell}}\right]\right\} \\
+\lambda_{m} \frac{h_{M^{*}}}{2}\left[k_{m}\left(t_{0}+(i+1) h_{M^{*}}, t_{0}\right) u_{M^{*}, 0}^{0}+2 \sum_{d=1}^{i} k_{m}\left(t_{0}+(i+1) h_{M^{*}}, t_{0}+d h_{M^{*}}\right) u_{M^{*}, d}^{0}\right. \\
\left.+k_{m}\left(t_{0}+(i+1) h_{M^{*}}, t_{0}(i+1) h_{M^{*}}\right) u_{M^{*}, i+1}^{0}\right] .
\end{gathered}
$$

Let $\quad \mathcal{A}_{k}^{\sigma, M^{*}}(s)=\frac{h_{M^{*}}^{-\sigma_{k-s}}}{\Gamma\left(2-\sigma_{k-s}\right)}$ and $\mathcal{H}_{k}^{\sigma, M^{*}}(i+1) p_{k}\left(t_{0}+(i+1) h_{M^{*}}\right)+\sum_{s=0}^{n-1} P_{s}\left(t_{0}+(i+1) h_{M^{*}}\right) \mathcal{A}_{k}^{\sigma, M^{*}}(s)$, $\forall k \in \mathbb{Z}^{+}, s=0,1, \ldots, k-1, \quad i=0,1, \ldots, 2^{M^{*}}-1$ with $\mathcal{A}_{k}^{\sigma, M^{*}}(k)=1, P_{0}\left(t_{0}+(i+1) h_{M^{*}}\right)=1$

$$
\begin{gathered}
u_{M^{*}, i+1}^{0}\left\{\mathcal{H}_{n}^{\alpha, M^{*}}(i+1)-\frac{h_{M^{*}}}{2} \sum_{\ell=0}^{m-1} \lambda_{\ell} k_{i+1, i+1}^{\ell, M^{*}, 0} \mathcal{A}_{m}^{\beta, M^{*}}(\ell)-\frac{\lambda_{m} h_{M^{*}}}{2} k_{i+1, i+1}^{m, M^{*}, 0}\right\} \\
=f_{i+1}^{M^{*}, 0}+\left\{\sum_{s=0}^{n-1} p_{s, i+1}^{0} \mathcal{A}_{n}^{\alpha, M^{*}}(s)-\frac{h_{M^{*}}}{2} \sum_{\ell=0}^{m-1} \lambda_{\ell} k_{i+1, i+1}^{\ell, M^{*}, 0} \mathcal{A}_{m}^{\beta, M^{*}}(\ell)\right\} u_{M^{*}, i}^{0} \\
-\sum_{j=1}^{i}\left[u_{M^{*}, i-j+1}^{0}-u_{M^{*}, i-j}^{0}\right]\left(\sum_{i i=0}^{n-1} P_{i i, i+1}^{0} \mathcal{A}_{n}^{\alpha, M^{*}}(i i) b_{j}^{\alpha_{n}-i i}\right) \\
+h_{M^{*}} \sum_{d=1}^{i} \sum_{j=0}^{d=1}\left[u_{M^{*}, d-j}^{0}-u_{M^{*}, d-j-1}^{0}\right]\left(\sum_{\ell=0}^{m-1} \lambda_{\ell} k_{i+1, d}^{\ell, M^{*}, 0} \mathcal{A}_{m}^{\beta, M^{*}}(\ell) b_{j}^{\beta_{m}-\ell}\right) \\
+\frac{h_{M^{*}}}{2} \sum_{j=1}^{i}\left[u_{M^{*}, i-j+1}^{0}-u_{M^{*}, i-j}^{0}\right]\left(\sum_{\ell=0}^{m-1} \lambda_{\ell} k_{i+1, i+1}^{\ell, M^{*}, 0} \mathcal{A}_{m}^{\beta, M^{*}}(\ell) b_{j}^{\beta_{m}-\ell}\right) \\
+\frac{\lambda_{m} h_{M^{*}}}{2} k_{i+1, i}^{m, M^{*}, 0} u_{M^{*}, i}^{0}+\lambda_{m} h_{M^{*}} \sum_{d=0}^{i} k_{i+1, d}^{m, M^{*}, 0} u_{M^{*}, d}^{0} .
\end{gathered}
$$

If $M^{*}=0, i=0$ and $h_{0}=\frac{h}{2^{0}}=h$, we obtain

$$
\begin{gathered}
u_{0,1}^{0}\left\{\mathcal{H}_{n}^{\alpha, 0}(1)-\frac{h_{0}}{2} \sum_{\ell=0}^{m-1} \lambda_{\ell} k_{1,1}^{\ell, 0} \mathcal{A}_{m}^{\beta, 0}(\ell)-\frac{\lambda_{m} h_{0}}{2} k_{1,1}^{m, 0}\right\} \\
=f_{1}^{0}+\left\{\sum_{s=0}^{n-1} p_{s, 1}^{0} \mathcal{A}_{n}^{\alpha, 0}(s)-\frac{h_{0}}{2} \sum_{\ell=0}^{m-1} \lambda_{\ell} k_{1,1}^{\ell, 0} \mathcal{A}_{m}^{\beta, 0}(\ell)\right\} u_{0,0}^{0}+\frac{h_{0}}{2} k_{1,0}^{m, 0,0} u_{0,0}^{0}
\end{gathered}
$$

So we can say $u_{0,1}^{0} \approx Q_{1}^{(p)}$, from this we come to the conclusion that the path normal quadratic spline function can be analyzed with two technique the first is using Trapezoid (NQST). And the second using First step of linear spline (using Richardson Extrapolation) (NQSL).

\subsection{Quadratic spline function using Extrapolation (VFID's)}

To sketching the quadratic spline proximity method for solving Volterra integro- fractional differential equation Eqns.(1) and (2) using extrapolation, the interval $[a, b]$. First divided the interval $[a, b]$ be into $N$-equal subintervals of length of $h=\frac{b-a}{N}$ with endpoints, the quadratic spline $Q(t)$ interpolating the function with Extrapolation in the interval $t \in\left[t_{r}+i h_{M^{*}}, t_{r}+(i+1) h_{M^{*}}\right]$ is specified by the formula

$$
\begin{gathered}
Q_{M^{*}, i}^{r}(t)=A_{M^{*}, i}^{r}(t) Q_{M^{*}, i}^{r}+B_{M^{*}, i}^{r}(t) Q_{M^{*}, i}^{r+1}+C_{M^{*}, i}^{r}(t) Q_{M^{*}, i}^{\left({ }^{\prime}\right) r} \\
r=0,1, \ldots, N-1, i=0,1, \ldots, 2^{M^{*}}-1, M^{*}=0,1, \ldots, M, h_{M^{*}}=\frac{h}{2^{M^{*}}}
\end{gathered}
$$


Where

$$
\begin{gathered}
A_{M^{*}, i}^{r}(t)=1-\left(\frac{t-\left(t_{r}+i h_{M^{*}}\right)}{h_{M^{*}}}\right)^{2}, B_{M^{*}, i}^{r}(t)=1-A_{M^{*}, i}^{r}(t)=\left(\frac{t-\left(t_{r}+i h_{M^{*}}\right)}{h_{M^{*}}}\right)^{2} \\
C_{M^{*}, i}^{r}(t)=\frac{\left(t-\left(t_{r}+i h_{M^{*}}\right)\right)\left(\left(t_{r}+(i+1) h_{M^{*}}\right)-t\right)}{h_{M^{*}}}
\end{gathered}
$$

Thus

$$
\begin{aligned}
&{ }_{a}^{C} D_{t}^{\alpha} Q_{M^{*}, i}^{r}(t) \quad \frac{-2}{h_{M^{*}}^{2}}\left[\frac{(t-a)^{2-\alpha}}{\Gamma(3-\alpha)}-\frac{(t-a)^{1-\alpha}}{\Gamma(2-\alpha)}\left(t_{r}+i h_{M^{*}}-a\right)\right] Q_{M^{*}, i}^{r} \\
&+\frac{2}{h_{M^{*}}^{2}}\left[\frac{(t-a)^{2-\alpha}}{\Gamma(3-\alpha)}-\frac{(t-a)^{1-\alpha}}{\Gamma(2-\alpha)}\left(t_{r}+i h_{M^{*}}-a\right)\right] Q_{M^{*}, i+1}^{r} \\
&-\frac{2}{h_{M^{*}}^{2}}\left[\frac{(t-a)^{2-\alpha}}{\Gamma(3-\alpha)}-\frac{(t-a)^{1-\alpha}}{\Gamma(2-\alpha)}\left(t_{r}+i h_{M^{*}}-a\right)\right] Q_{M^{*}, i+1}^{r} \\
&-\frac{2}{h_{M^{*}}^{2}}\left[\frac{(t-a)^{2-\alpha}}{\Gamma(3-\alpha)}-\left(t_{r}+\left(i+\frac{1}{2}\right) h_{M^{*}}-a\right) \frac{(t-a)^{1-\alpha}}{\Gamma(2-\alpha)}\right] Q_{M^{*}, i}^{\left(^{\prime}\right) r}
\end{aligned}
$$

From Eqn.(1) putting $t=t_{r}+(i+1) h_{M^{*}}$, then collocate Eqn.(5) at the uniform grid points after substituting Eqn.(12) into Eqn.(5) then we obtain

$$
\begin{aligned}
& \frac{2 h_{M^{*}}^{2-\alpha_{n}}\left(2^{M^{*}} r+i+1\right)^{1-\alpha_{n}}}{h_{M^{*}}^{2} \Gamma\left(3-\alpha_{n}\right)}\left[2^{M^{*}} r\left(\alpha_{n}-1\right)+i\left(\alpha_{n}-1\right)+1\right]\left[Q_{M^{*}, i+1}^{r}-Q_{M^{*}, i}^{r}\right] \\
& -\frac{2 h_{M^{*}}^{2-\alpha_{n}}\left(2^{M^{*}} r+i+1\right)^{1-\alpha_{n}}}{h_{M^{*}} \Gamma\left(3-\alpha_{n}\right)}\left[2^{M^{*}} r\left(\alpha_{n}-1\right)+i\left(\alpha_{n}-1\right)+\frac{1}{2} \alpha_{n}\right] Q_{M^{*}, i}^{\left(^{\prime}\right) r} \\
& +\sum_{i i=1}^{n-1} P_{i i}\left(t_{r}+(i+1) h_{M^{*}}\right)\left\{\frac{2 h_{M^{*}}^{2-\alpha_{n-i i}}\left(2^{M^{*}} r+i+1\right)^{1-\alpha_{n-i i}}}{h_{M^{*}}^{2} \Gamma\left(3-\alpha_{n-i i}\right)}\right. \\
& \left.\left[2^{M^{*}} r\left(\alpha_{n-i i}-1\right)+i\left(\alpha_{n-i i}-1\right)+1\right]\left[Q_{M^{*}, i+1}^{r}-Q_{M^{*}, i}^{r}\right]\right\}-\frac{2 h_{M^{*}}^{2-\alpha_{n-i i}}\left(2^{M^{*}} r+i+1\right)^{1-\alpha_{n-i i}}}{h_{M^{*}} \Gamma\left(3-\alpha_{n-i i}\right)} \\
& {\left[2^{M^{*}} r\left(\alpha_{n-i i}-1\right)+i\left(\alpha_{n-i i}-1\right)+\frac{1}{2} \alpha_{n-i i}\right] Q_{M^{*}, i}^{\left({ }^{\prime}\right)}+p_{n}\left(t_{r}+(i+1) h_{M^{*}}\right) Q_{M^{*}, i+1}^{r}} \\
& =f\left(t_{r}+(i+1) h_{M^{*}}\right)+\sum_{\ell=0}^{m-1} \lambda_{\ell}\left[\sum _ { j = 0 } ^ { r - 1 } \int _ { t _ { j } } ^ { t _ { j + 1 } } k _ { \ell } ( t _ { r } + ( i + 1 ) h _ { M ^ { * } } , s ) \left[{ }_{a}^{C} D_{s}^{\beta_{m-\ell}} A_{j}(s) Q_{j}^{C}\right.\right. \\
& \left.\left.+{ }_{a}^{C} D_{s}^{\beta_{m-\ell}} B_{j}(s) Q_{j+1}^{C}+{ }_{a}^{C} D_{s}^{\beta_{m-\ell}} C_{j}(s) Q_{j}^{\left({ }^{\prime}\right), C}\right] d s\right] \\
& +\sum_{j=0}^{i-1} \int_{t_{r}+j h_{M^{*}}}^{t_{r}+(j+1) h_{M^{*}}} k_{\ell}\left(t_{r}+(i+1) h_{M^{*}}, s\right)\left[{ }_{a}^{C} D_{s}^{\beta_{m-\ell}} A_{M^{*}, j}^{r}(s) Q_{M^{*}, j}^{r}\right. \\
& \left.+{ }_{a}^{C} D_{s}^{\beta_{m-\ell}} B_{M^{*}, j}^{r}(s) Q_{M^{*}, j+1}^{r}+{ }_{a}^{C} D_{s}^{\beta_{m-\ell}} C_{M^{*}, j}^{r}(s) Q_{M^{*}, j}^{\left({ }^{\prime}\right), r}\right] d s \\
& +\int_{t_{r}+i h_{M^{*}}}^{t_{r}+(i+1) h_{M^{*}}} k_{\ell}\left(t_{r}+(i+1) h_{M^{*}}, s\right) \\
& {\left[{ }_{a}^{C} D_{s}^{\beta_{m-\ell}} A_{M^{*}, i}^{r}(s) Q_{M^{*}, i}^{r}+{ }_{a}^{C} D_{s}^{\beta_{m-\ell}} B_{M^{*}, i}^{r}(s) Q_{M^{*}, i+1}^{r}+{ }_{a}^{C} D_{s}^{\beta_{m-\ell}} C_{M^{*}, i}^{r}(s) Q_{M^{*}, i}^{\left({ }^{\prime}\right), r}\right] d s} \\
& \lambda_{m}\left[\sum_{j=0}^{r-1} \int_{t_{j}}^{t_{j+1}} k_{m}\left(t_{r}+(i+1) h_{M^{*}}, s\right)\left[A_{j}(s) Q_{j}^{C}+B_{j}(s) Q_{j+1}^{C}+C_{j}(s) Q_{j}^{\left({ }^{\prime}\right), C}\right] d s\right] \\
& +\sum_{j=0}^{i-1} \int_{t_{r}+j h_{M^{*}}}^{t_{r}+(j+1) h_{M^{*}}} k_{m}\left(t_{r}+(i+1) h_{M^{*}}, s\right)\left[A_{M^{*}, j}^{r}(s) Q_{M^{*}, j}^{r}+B_{M^{*}, j}^{r}(s) Q_{M^{*}, j+1}^{r}+C_{M^{*}, j}^{r}(s) Q_{M^{*}, j}^{\left({ }^{\prime}\right), r}\right] d s \\
& +\int_{t_{r}+i h_{M^{*}}}^{t_{r}+(i+1) h_{M^{*}}} k_{m}\left(t_{r}+(i+1) h_{M^{*}}, s\right)\left[A_{M^{*}, i}^{r}(s) Q_{M^{*}, i}^{r}+B_{M^{*}, i}^{r}(s) Q_{M^{*}, i+1}^{r}+C_{M^{*}, i}^{r}(s) Q_{M^{*}, i}^{\left(^{\prime}\right), r}\right] d s
\end{aligned}
$$

Let

$$
\mathcal{W}_{M^{*}, i}^{n, r}(s)=P_{s\left(t_{r}+(i+1) h_{M^{*}}\right)} \frac{2 h_{M^{*}}^{2-\alpha_{n-s}}\left(2^{M^{*}} r+i+1\right)^{1-\alpha_{(n-s)}}}{h_{M^{*}}^{2} \Gamma\left(3-\alpha_{(n-s)}\right)}\left[2^{M^{*}} r\left(\alpha_{(n-s)}-1\right)+i\left(\alpha_{(n-s)}-1\right)+1\right]
$$




$$
\begin{gathered}
\mathcal{V}_{M^{*}, i}^{n, r}(s)= \\
P_{s\left(t_{r}+(i+1) h_{M^{*}}\right)} \frac{2 h_{M^{*}}^{2-\alpha_{n-s}}\left(2^{M^{*}} r+i+1\right)^{1-\alpha_{(n-s)}}}{h_{M^{*}} \Gamma\left(3-\alpha_{(n-s)}\right)}\left[2^{M^{*}} r\left(\alpha_{(n-s)}-1\right)+i\left(\alpha_{(n-s)}-1\right)+\frac{1}{2} \alpha_{(n-s)}\right] \\
\mathcal{H}_{M^{*}, i}^{n, r}(s)=P_{s\left(t_{r}+(i+1) h_{M^{*}}\right)}+\sum_{s=0}^{n-1} \mathcal{W}_{M^{*}, i}^{n, r}(s), r=0,1, \ldots, N-1, s=0,1, \ldots, n-1 .
\end{gathered}
$$

$i=0,1, \ldots, 2^{M^{*}}-1, M^{*}=0,1, \ldots, M . h_{M^{*}}=\frac{h}{2^{M^{*}}}, \forall M \in \mathbb{Z}^{+} \cup\{0\}$, then Eqn(13) becomes

$$
\begin{aligned}
& Q_{M^{*}, i+1}^{r}\left\{\mathcal{H}_{M^{*}, i}^{n, r}(s)-\sum_{\ell=0}^{m-1} \lambda_{\ell} \int_{t_{r}+i h_{M^{*}}}^{t_{r}+(i+1) h_{M^{*}}} k_{\ell}\left(t_{r}+(i+1) h_{M^{*}}, s\right)_{a}^{C} D_{s}^{\beta_{m-\ell}} B_{M^{*}, j}^{r}(s) d s\right. \\
& \left.-\lambda_{m} \int_{t_{r}+i h_{M^{*}}}^{t_{r}+(i+1) h_{M^{*}}} k_{m}\left(t_{r}+(i+1) h_{M^{*}}, s\right) B_{M^{*}, j}^{r}(s) d s\right\} \\
& =f\left(t_{r}+(i+1) h_{M^{*}}\right)+Q_{M^{*}, i}^{r}\left\{\sum_{s=0}^{n-1} \mathcal{W}_{M^{*}, i}^{n, r}(s)\right. \\
& +\sum_{\ell=0}^{m-1} \lambda_{\ell} \int_{t_{r}+i h_{M^{*}}}^{t_{r}+(i+1) h_{M^{*}}} k_{\ell}\left(t_{r}+(i+1) h_{M^{*}}, s\right)_{a}^{C} D_{s}^{\beta_{m-\ell}} A_{M^{*}, i}^{r}(s) d s \\
& \left.+\lambda_{m} \int_{t_{r}+i h_{M^{*}}}^{t_{r}+(i+1) h_{M^{*}}} k_{m}\left(t_{r}+(i+1) h_{M^{*}}, s\right) A_{M^{*}, j}^{r}(s) d s\right\} \\
& +Q_{M^{*}, i}^{\left({ }^{\prime}\right) r}\left\{\sum_{s=0}^{n-1} \mathcal{V}_{M^{*}, i}^{n, r}(s)+\sum_{\ell=0}^{m-1} \lambda_{\ell} \int_{t_{r}+i h_{M^{*}}}^{t_{r}+(i+1) h_{M^{*}}} k_{\ell}\left(t_{r}+(i+1) h_{M^{*}}, s\right)_{a}^{C} D_{s}^{\beta_{m-\ell}} C_{M^{*}, i}^{r}(s) d s\right. \\
& \left.+\lambda_{m} \int_{t_{r}+i h_{M^{*}}}^{t_{r}+(i+1) h_{M^{*}}} k_{m}\left(t_{r}+(i+1) h_{M^{*}}, s\right) C_{M^{*}, i}^{r}(s) d s\right\} \\
& +\sum_{\ell=0}^{m-1} \lambda_{\ell}\left[\sum _ { j = 0 } ^ { n - 1 } \int _ { t _ { j } } ^ { t _ { j + 1 } } k _ { \ell } ( t _ { r } + ( i + 1 ) h _ { M ^ { * } } , s ) \left[{ }_{a}^{C} D_{s}^{\beta_{m-\ell}} A_{j}(s) Q_{j}^{c}\right.\right. \\
& \left.+{ }_{a}^{C} D_{s}^{\beta_{m-\ell}} B_{j}(s) Q_{j+1}^{C}(s)+{ }_{a}^{C} D_{s}^{\beta_{m-\ell}} C_{j}(s) Q_{j}^{\left({ }^{\prime}\right), C}\right] d s \\
& \sum_{j=0}^{i-1} \int_{t_{r}+j h_{M^{*}}}^{t_{r}+(j+1) h_{M^{*}}} k_{\ell}\left(t_{r}+(i+1) h_{M^{*}}, s\right)\left[{ }_{a}^{C} D_{s}^{\beta_{m-\ell}} A_{M^{*}, j}^{r}(s) Q_{M^{*}, j}^{r}\right. \\
& \left.\left.+{ }_{a}^{C} D_{s}^{\beta_{m-\ell}} B_{M^{*}, j}^{r}(s) Q_{M^{*}, j+1}^{r}(s)+{ }_{a}^{C} D_{s}^{\beta_{m-\ell}} C_{M^{*}, j}^{r}(s) Q_{M^{*}, j}^{\left({ }^{\prime}\right), r}\right] d s\right] \\
& +\lambda_{m}\left[\sum _ { j = 0 } ^ { n - 1 } \int _ { t _ { j } } ^ { t _ { j + 1 } } k _ { m } ( t _ { r } + ( i + 1 ) h _ { M ^ { * } } , s ) \left[A_{j}(s) Q_{j}^{c}\right.\right. \\
& \left.+B_{j}(s) Q_{j+1}^{C}+C_{j}(s) Q_{j}^{\left({ }^{\prime}\right), C}\right] d s+\sum_{j=0}^{i-1} \int_{t_{r}+j h_{M^{*}}}^{t_{r}+(j+1) h_{M^{*}}} k_{m}\left(t_{r}+(i+1) h_{M^{*}}, s\right) \\
& \left.\left[A_{M^{*}, j}^{r}(s) Q_{M^{*}, j}^{r}+B_{M^{*}, j}^{r}(s) Q_{M^{*}, j+1}^{r}(s)+C_{M^{*}, j}^{r}(s) Q_{M^{*}, j}^{('), r}\right] d s\right]
\end{aligned}
$$

Eqn.(14) is obtained to find the approximate solution $Q_{M^{*}, i+1}^{r}, \forall r=0,1, \ldots, N-1, \forall i=0,1, \ldots, 2^{M^{*}}-1$, $M^{*}=0,1, \ldots, M, h_{M^{*}}=\frac{h}{2^{M^{*}}}, \forall M \in \mathbb{Z}^{+} \cup\{0\}$. For finding $Q_{0}^{\prime}$ by forward formula $Q_{0}^{\prime}=\frac{Q_{1}^{(p)}-Q_{0}}{h}, Q_{0}$ is the initial condition Eqn.(2), using for next iterations $Q_{r}^{\prime}+Q_{r-1}^{\prime}=\frac{2\left(Q_{r}-Q_{r-1}\right)}{h}, r=1,2, \ldots, N$, in this technique we need to find $Q_{1}^{(p)}$ so in the same way such as how through from Eqn.(9) or Eqn.(11), from this we come to the conclusion that the path Extrapolation quadratic spline function can be analyzed with two technique the first is using trapezoid (EQST). And the second using first step of linear spline (using Richardson Extrapolation) (EQSL).

\section{Numerical scheme}

In this section, we present two examples in which their numerical results. In examples (1) and (2), we have compared the results of this method for (VIFDE's) of the second kind with collocation normal spline and Richard Extrapolation method the result shown in tables $(1,3)$ an almost large interval to show capability of the 
method shown in table (4) for example 2. Finally, demonstrates figures $(1,2)$ to compared numerical and the exact solution of the examples (1) and (2) where $h=0.1$.

Example 1. Consider the linear VIFDE on $0 \leq t \leq 1$ :

$$
\begin{gathered}
{ }_{0}^{C} D_{t}^{0.7} u(t)+t_{0}^{C} D_{t}^{0.2}-2 u(t)= \\
=f(t)+\int_{0}^{t}\left[\left(t-2 s^{2}\right)_{0}^{C} D_{t}^{0.3} u(s)+(t-s)_{0}^{C} D_{t}^{0.1} u(s)-(t s-1) u(s)\right] d s,
\end{gathered}
$$

where

$$
f(t)=-\frac{2}{3} t^{4}+\frac{1}{2} t^{3}+t^{2}-t-\frac{2}{\Gamma(1.3)} t^{0.3}-\frac{2}{\Gamma(1.8)}-2(1-2 t)+\frac{20}{629 \Gamma(1.7)} t^{2.7}(37-34 t) .
$$

With the initial condition: $u(0)=1$, where the exact solution is given by $u(t)=1-2 t$.

Example 2. Consider the linear VIFDE on $0 \leq t \leq 1$ :

$$
\begin{gathered}
{ }_{0}^{C} D_{t}^{2 \beta} u(t)-\frac{1}{2}{ }_{0}^{C} D_{t}^{\beta}+\left(1+t^{3}\right) u(t)= \\
=f(t)+\int_{0}^{t}\left[t s_{0}^{C} D_{t}^{2 \beta} u(s)+\left(t^{2}-s\right)_{0}^{C} D_{t}^{\beta} u(s)+e^{t+s} u(s)\right] d s,
\end{gathered}
$$

where

$$
\begin{aligned}
f(t)=e^{t}-e^{2 t}(t-1)^{2}+t^{5}-t^{3}+t^{2}-1 & +\frac{1}{\Gamma(3-2 \beta)}\left(2-\frac{1}{2-\beta} t^{3}\right) t^{2-2 \beta} \\
& +\frac{1}{\Gamma(3-\beta)}\left(\frac{2}{4-\beta} t^{2}-1\right) t^{2-\beta}-\frac{2}{\Gamma(4-\beta)} t^{5-\beta} .
\end{aligned}
$$

With the initial condition: $u(0)=-1$, where the exact solution of this problem is known $u(t)=t^{2}-1$, and $\beta=0.5$.

Table 1

Exact and numerical solution of example 1

\begin{tabular}{|c|c|c|c|c|c|}
\hline$t$ & Exact & \multicolumn{5}{|c|}{$N=10$} \\
\hline & & NQST & NQSL & EQST $(m=2)$ & EQSL $(m=2)$ \\
\hline 0 & 1 & 1.0 & 1.0 & 1.0 & 1.0 \\
\hline 0.1 & 0.8 & 0.8000004930653 & 0.8 & 0.80000000707939 & 0.8 \\
\hline 0.2 & 0.6 & 0.6000007031481 & 0.6 & 0.60000071148317 & 0.6 \\
\hline 0.3 & 0.4 & 0.4000010136467 & 0.4 & 0.40000222633954 & 0.4 \\
\hline 0.4 & 0.2 & 0.2000013769366 & 0.2 & 0.20000484834216 & 0.2 \\
\hline 0.5 & 0.0 & 0.0000017667637 & $2.496349159 e^{-17}$ & 0.0000084819165 & $7.57181976631 e^{-19}$ \\
\hline 0.6 & -0.2 & -0.1999978313918 & -0.2 & -0.19998695732248 & -0.2 \\
\hline 0.7 & -0.4 & -0.39999742490777 & -0.4 & -0.39998156309772 & -0.4 \\
\hline 0.8 & -0.6 & -0.59999701665405 & -0.6 & -0.59997541684731 & -0.6 \\
\hline 0.9 & -0.8 & -0.79999660662054 & -0.8 & -0.79996858034163 & -0.8 \\
\hline 1.0 & -1 & -0.99999619296744 & -1.0 & -0.99996109404 & -1.0 \\
\hline \hline L.S.E & & $5.3025254 e-11$ & $6.2317591 e-34$ & $3.7161423 e-9$ & $5.7332455 e-37$ \\
\hline R.Time/Sec & & 40.994059 & 51.198122 & 302.24999 & 419.6619 \\
\hline
\end{tabular}

The result in Table (2) shows R-Extrapolation technique for solving quadratic spline methods using trapezoidal and first step of linear spline for $h=0.05(N=20)$. For Example 1 . 
Approximate Solution of Volterra...

Table 2

The comparison of the solution R-Extrapolation technique

\begin{tabular}{|c|c|c|c|c|c|}
\hline$t$ & Exact & \multicolumn{5}{|c|}{$N=20$} \\
\hline & & EQST $(m=2)$ & EQSL $(m=2)$ & EQST $(m=3)$ & EQSL $(m=3)$ \\
\hline 0 & 1 & 1.0 & 1.0 & 1.0 & 1.0 \\
\hline 0.1 & 0.8 & 0.800000008905707 & 0.8 & 0.800000006359992 & 0.8 \\
\hline 0.2 & 0.6 & 0.600000050701418 & 0.6 & 0.600000045813416 & 0.6 \\
\hline 0.3 & 0.4 & 0.400000151375986 & 0.4 & 0.400000137044427 & 0.4 \\
\hline 0.4 & 0.2 & 0.20000031526505 & 0.2 & 0.200000284526254 & 0.2 \\
\hline 0.5 & 0.0 & 0.00000053995763 & $1372334369 e^{-18}$ & 0.0000004860751345 & $-7.73860979575 e^{-19}$ \\
\hline 0.6 & -0.2 & -0.199999179770634 & -0.2 & -0.199999262960361 & -0.2 \\
\hline 0.7 & -0.4 & -0.399998849511254 & -0.4 & -0.399998967571431 & -0.4 \\
\hline 0.8 & -0.6 & -0.599998474114693 & -0.6 & -0.599998632098907 & -0.6 \\
\hline 0.9 & -0.8 & -0.799998057255924 & -0.8 & -0.799998259837467 & -0.8 \\
\hline 1.0 & -1 & -0.999997601330418 & -1.0 & -0.999997852936052 & -1.0 \\
\hline \hline \multicolumn{2}{|c|}{ L.S.E } & $1.4269108 e-11$ & $4.5677668 e-36$ & $1.1456484 e-11$ & $5.9886082 e-37$ \\
\hline R.Time/Sec & 8555.47371 & 2374.498 & 2413.3623 & 2398.2578 \\
\hline
\end{tabular}

Table 3

Exact and numerical solution of example 2

\begin{tabular}{|c|c|c|c|c|c|}
\hline$t$ & Exact & \multicolumn{5}{|c|}{$N=10$} \\
\hline & & NQST & NQSL & EQST $(m=2)$ & EQSL $(m=2)$ \\
\hline 0 & 1 & 1.0 & 1.0 & 1.0 & 1.0 \\
\hline 0 & -1.0 & -1.0 & -1.0 & -1.0 & -1.0 \\
\hline 0.1 & -0.99 & -0.984097358703339 & -0.98385921585012 & -0.991476290775673 & -0.991479158909235 \\
\hline 0.2 & -0.96 & -0.956638239698859 & -0.95627586881473 & -0.965258118725224 & -0.965263505489059 \\
\hline 0.3 & -0.91 & -0.914517584731656 & -0.914089026848564 & -0.921013271619213 & -0.921018780914492 \\
\hline 0.4 & -0.84 & -0.856459372872957 & -0.855994041063225 & -0.859259280306947 & -0.859264546925167 \\
\hline 0.5 & -0.75 & -0.782066581154528 & -0.781577394923225 & -0.780434507191968 & -0.780439517960847 \\
\hline 0.6 & -0.64 & -0.691478975785645 & -0.690968031530877 & -0.685006055226451 & -0.685010914123675 \\
\hline 0.7 & -0.51 & -0.585278870476111 & -0.584740180555245 & -0.573535077158801 & -0.57353994630581 \\
\hline 0.8 & -0.36 & -0.464504501114389 & -0.463925265789229 & -0.446739340155018 & -0.446744421203363 \\
\hline 0.9 & -0.19 & -0.330716434084407 & -0.330077470104528 & -0.305564188908158 & -0.305569718588994 \\
\hline 1.0 & 0 & -0.186096022057057 & -0.185371604481513 & -0.151269656763997 & -0.151275909797876 \\
\hline \hline \multicolumn{7}{|c|}{ L.S.E } & 0.075036756 & 0.074289865 & 0.051271853 & 0.051277655 \\
\hline R.Time/Sec & 46.939625 & 53.091482 & 355.20677 & 297.93127 \\
\hline
\end{tabular}

The result in table (4) shows the least-square errors and running times (elapsed time) for quadratic spline methods with different values of steps size $h$. For Example 2.

Table 4

Comparision for different value of $\mathrm{N}$

\begin{tabular}{|c|c|c|c|c|c|c|}
\hline $\mathrm{h}$ & \multicolumn{2}{|c|}{$0.1(N=10)$} & \multicolumn{2}{c|}{$0.05(N=20)$} & \multicolumn{2}{c|}{$0.033(N=30)$} \\
\hline Quadratic & L.S.E & R.Time $/$ Sec & L.S.E & R.Time $/$ Sec & L.S.E & R.Time $/$ Sec \\
\hline NQSL & 0.074289865 & 53.091482 & 0.066459532 & 136.90037 & 0.059164974 & 308.13296 \\
\hline EQSL $(m=2)$ & 0.051277655 & 297.93127 & 0.046061105 & 1115.3817 & 0.044419219 & 2788.2102 \\
\hline EQSL $(m=3)$ & 0.045270078 & 1908.428 & 0.043314536 & 3855.422 & 0.04268915 & 8619.3941 \\
\hline
\end{tabular}




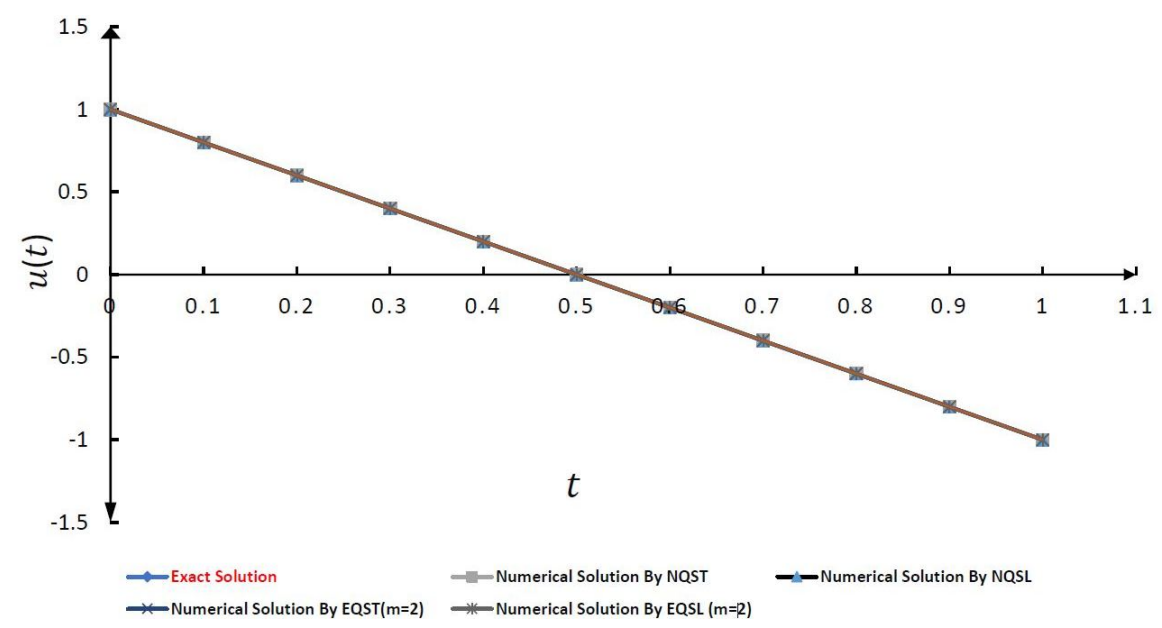

Figure 1. Compared numerical and the exact solution of the example $1, h=0.1$.

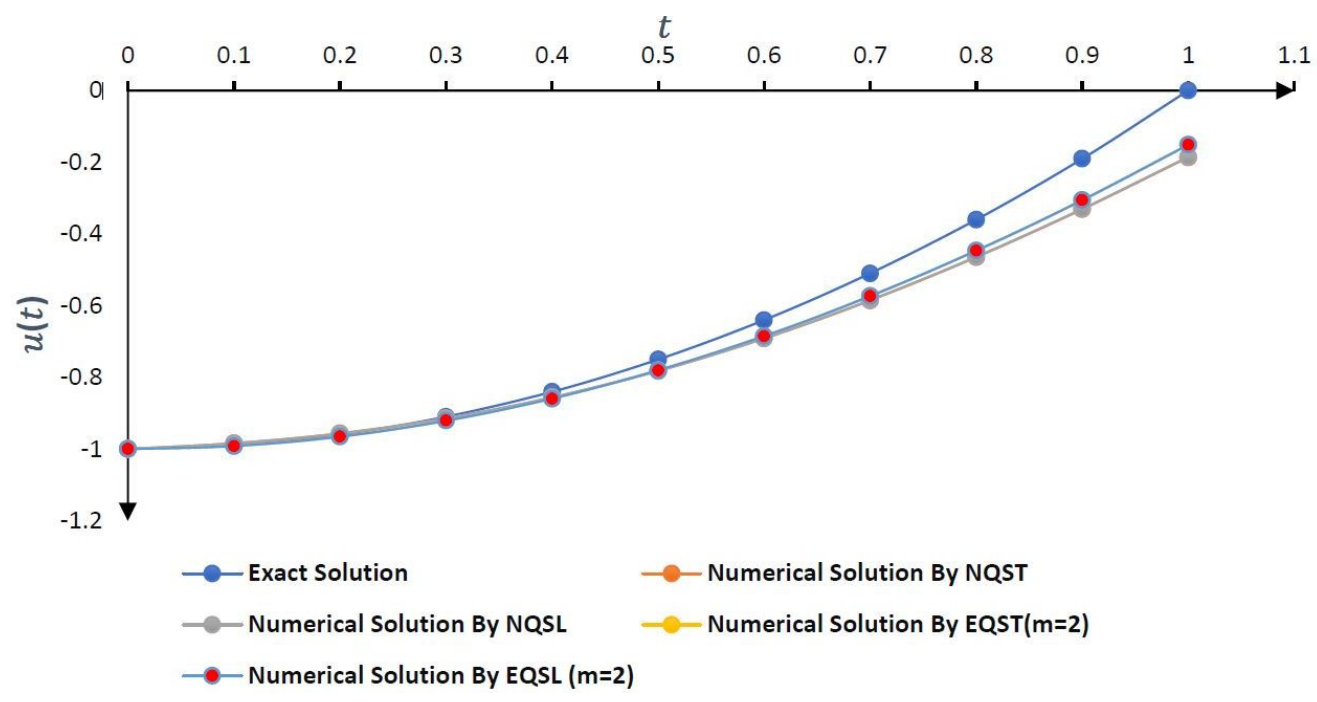

Figure 2. Compared numerical and the exact solution of the example $2, h=0.1$.

\section{Conclusion}

In this work, we have fully attempted to find the numerical solution of the Volterra integro-fractional differential equations (VIFDE's) by using quadratic spline approximate. The numerical procedure and methodology are done in a very straightforward and effective manner. Through the numerical calculation, we confirmed that the Richardson Extrapolation method has the highest degree of accuracy. On the basis of this work, tables (2) and (4) displayed comparison between normal quadratic spline and using Extrapolation method with different step sizes. Furthermore, interpolating quadratic spline for linear function is closer to the quadratic function as displayed in tables (1) and (3). Likewise, new techniques using the first derivative at the initial point by the First step of linear spline(using R-Extrapolation technique), then our solution would be better and the ration of mistake would be fewer in comparison of the method of Trapezoidal at finding first derivative at the initial point. Figures (1) and (2) represents which one is the best technique for solving (VIFDE's).

\section{References}

1 Ricardo, A. (2017). A Caputo fractional derivative of a function with respect to another function. Communications in Nonlinear Science and Numerical Simulation, 44, 460-481.

DOI.org/10.1016/j.cnsns.2016.09.006. 
2 Tenreiro, J., Silva, M., Barbosa, R. , Jesus, I., Reis, C., Marcos, M., \& Galhano, A. (2010). Some Applications of Fractional Calculus in Engineering. Mathematical Problems in Engineering, 1-34.

3 Guo, B., Pu, X., \& Huang, F. (2015). Fractional Partial Differential Equations and Their Numerical Solutions. World Scientific Publishing Co: Pte. Ltd.

4 Guang-hua, G., Zhi-Zhong, S., \& Hong-Wei, Zh. (2014). A new fractional numerical differentiation formula to approximate the Caputo fractional derivative and its applications. Journal of Computa-tional Physics, 259, 33-50. DOI.org/10.1016/j.jcp.2013.11.017.

5 Mustafa, A.D., Ercan C., Hasan, B., \& Haci, M. (2018). Cancer treatment model with the Caputo-Fabrizio fractional Derivative. The European physical journal plus, 133, 92.

DOI 10.1140/epjp/i2018-11950-y.

6 Shazad, Sh.A. (2009). On system of linear volterra integro-fractional differential equations. Ph.D. dissertation, University of Sulaimani.

7 Hamid, M., Hamid, S., Abolfazl, Gh., \& Yones, E. (2019). The Cubic B-spline Operational Matrix Based on Haar Scaling Functions for Solving Varieties of the Fractional Integro-differential Equations. Journal of Mathematics, 51(8), 45-65.

8 Shazad, Sh.A., \& Shokhan, A.H. (2011). Generalized taylor matrix method for solving linear integrofractional differential equations of volterra type. Applied Mathematical Sciences, 5, 1765-1780.

9 Khosrow, M., Reza, M., \& Paria, M. (2013). Numerical Solution of Volterra Functional Integral Equation by Using Cubic B-Spline Scaling Functions. Numerical Methods for Partial Differential Equations, 30(2), 699-722. DOI: $10.1002 /$ num.21837.

10 Shazad, Sh.A., Shokhan, A.H., \& Mariwan, R.A. (2019). Laplace adomian and laplace modified adomian decomposition methods for solving nonlinear integro-fractional differential equations of the volterrahammerstein type. Iraqi Journal of Science, 60, 2207-2222.

11 Shazad, Sh.A., \& Bzhween, A.S. (2019). Generalized Taylor Matrix Method for Solving Multi-Higher Nonlinear Integro-Fractional Differential Equations of Fredholm Type. ZANCO Journal of Pure and Applied Sciences, 2218-0230. DOI: http://dx.doi.org/10.21271/zjpas.

12 Rajani, B.D., \& Debasish, D. ( 2011). A Mixed Quadrature Rule by Blending Clenshaw-Curtis and Gauss-Legendre Quadrature Rules for Approximation of Real Definite Integrals in Adaptive Environment. Proceedings of the International MultiConference of Engineers and Computer Scientists, 1, 16-18.

13 Istvan, F., Agnes, H., \& Zahari, Z. (2010). Efficient implementation of stable Richardson Extrapolation algorithms. Computers and Mathematics with Applications, 60, 2309-2325. DOI.org/10.1016/j.camwa. 2010.08.025.

14 Michael, F., Tom, L., Marie, M., Knut, M., Larry, L., \& Schumaker. (2012). Mathematical Methods for Curves and Surfaces. 8th International Conference, Oslo, Norway.

15 Burden, R., \& Faires, J. (2011). Numerical Analysis. 9th ed., USA: Brooks/Cole. (34).

\author{
К.Х.Ф. Джвамер, Ш.Ш. Ахмед, Д.Х. Абдулла
}

\title{
Квадраттық сплайн-функциясын қолдана отырып, Вольтеррдің интегро-бөлшекті дифференциалдық теңдеулерінің жуық шешімі
}

\footnotetext{
Мақалада қалыпты квадраттық сплайн-функциясына негізделген Вольтеррдің интегро-бөлшектідифференциалдық теңдеудің шешімін жуықтаудың екі жаңа әдісі ұсынылған, ал екінші әдісте дискретті коллокация нүктелерін қолдана отырып, Ричардсонның экстраполяция әдісі қолданылған. Бөлшекті туындылары Капуто түсінігінде қарастырылды. Олардың дәлелдерімен қатар, Ричардсонның экстраполяция нүктелеріне Капуто туындысының ақырлы айырмашылығын қолдану үшін жаңа теорема енгізілді. Бастапқы нүктеде бірінші туындыны қолданатын жаңа әдістер негізінде келесі екі жағдай алынған: біріншісінде трапеция ережесі, екіншісінде Ричардсонның экстраполяция әдісі негізінде сызықтық сплайн-функциясының бірінші қадамы қолданылған. Атап айтқанда, бағдарлама Matlab (R2018b) талдау мысалдарында келтірілген. Әдістердің өнімділігі мен сенімділігін көрсететін сандық мысалдар бар, сонымен қатар, осы теңдеулер үшін қажетті интегралдарды есептеу үшін Кертис Кленшоу ережесі қолданылды.
} 
Kiлm сөздер: интегро-бөлшекті дифференциалдық теңдеу, Капуто туындысы, квадраттық сплайн, экстраполяция әдісі, Кленшоу.

К.Х.Ф. Джвамер, Ш.Ш. Ахмед, Д.Х. Абдулла

\section{Приближенное решение интегро-дробных дифференциальных уравнений Вольтерра с использованием квадратичной сплайн-функции}

В статье предложены два новых метода аппроксимации решения интегро-дробно-дифференциального уравнения Вольтерра (VIFDE), основанные на нормальной квадратичной сплайн-функции, а в основе второго метода лежит метод экстраполяции Ричардсона с использованием дискретных точек коллокации. Дробные производные рассмотрены в восприятии Капуто. Вместе с их доказательством введена новая теорема для точек экстраполяции Ричардсона для использования конечно-разностной аппроксимации производной Капуто. Новые методы с использованием первой производной в начальной точке таковы, что получены следующие два случая: первый с использованием правила трапеции, а второй - с учетом первого шага линейной сплайн-функции методом экстраполяции Ричардсона. В частности, программа приведена в примерах анализа в Matlab (R2018b). Имеются числовые примеры, чтобы продемонстрировать продуктивность и надежность методов, а также следовать правилу Кленшоу Кертиса для вычисления требуемых интегралов для этих уравнений.

Ключевые слова: интегро-дробное дифференциальное уравнение, производная Капуто, квадратичный сплайн, метод экстраполяции, Кленшоу. 


\author{
M.T. Kosmakova ${ }^{1}$, S.A. Iskakov ${ }^{1, *}$, L.Zh. Kasymova ${ }^{1,2}$ \\ ${ }^{1}$ Buketov Karaganda university, Karaganda, Kazakhstan; \\ ${ }^{2}$ Karaganda technical university, Karaganda, Kazakhstan \\ (E-mail: svetlanamir578@gmail.com, isagyndyk@mail.ru,l.kasymova2017@mail.ru)
}

\title{
To solving the fractionally loaded heat equation
}

\begin{abstract}
In this paper we consider a boundary value problem for a fractionally loaded heat equation in the class of continuous functions. Research methods are based on an approach to the study of boundary value problems, based on their reduction to integral equations. The problem is reduced to a Volterra integral equation of the second kind by inverting the differential part. We also carried out a study the limit cases for the fractional derivative order of the term with a load in the heat equation of the boundary value problem. It is shown that the existence and uniqueness of solutions to the integral equation depends on the order of the fractional derivative in the loaded term.
\end{abstract}

Keywords: loaded equation, fractional derivative, heat equation, Volterra integral equation, special function.

\section{Introduction}

The study of fractional differential equations was actively carried out as in previous decades [1-4], and now interest in this area continues to grow [5-7]. This is due both to the development of the fractional integration and differentiation theory, as well as applications of the apparatus of fractional integration and differentiation in various fields of science. The physical interpretation for fractional differential equations was considered in [3] from the point of view of the Riemann-Liouville's derivatives, as well as in [4]. In [6] a boundary value problem with integral conditions is considered for one class of fractional differential equations involving impulses. Some results of the existence of a solution for higher order differential equations with integral conditions can be found in [5]. Also an important section in the theory of differential equations is the class of loaded equations: $K u=L u(x)+M u(x)=f(x)$ in a domain $Q$ from $R^{n}$, where $L$ is a differential operator, and $M$ is a differential or integro-differential operator, including the operation of taking the trace of the function $u(x)$ on manifolds from the closure Q of dimension strictly less than $n$. Solving many important problems, for example, on the optimal management of the agroecosystem, is reduced to the study of such equations. In [8] on numerous examples A.M. Nakhushev showed the practical and theoretical importance of studies on loaded equations. In the papers of M.T. Jenaliev and students of his scientific school, the theory of loaded equations was further developed [9-12]. In [11], [12] loaded differential equations are interpreted as weak or strong perturbations of differential equations.

Of interest are boundary value problems for the fractionally loaded heat equation when the loaded term is presented in the form of a fractional derivative. The goal of papers [13-14] is to clarify the character of the fractional load on the solvability issues of the first boundary value problem for the heat equation, the load moves with a constant velocity. The loaded term is the trace of the fractional order derivative on the manifold $x=t$, namely, the loaded term is represented as a Riemann-Liouville fractional derivative. The resulting Volterra singular integral equation has a nonempty spectrum for certain values of the fractional derivative order. In the papers [15-16] the loaded term is represented in the form of the Caputo fractional derivative with respect to the time variable and the spatial variable, and the order of the derivative in the loaded term is less than the order of the differential part.

In this paper, we study a boundary value problem for a fractionally loaded heat equation (the loaded term of the equation is represented as a Riemann-Liouville fractional derivative, the load moves according to an arbitrary law). The boundary value problem is reduced to a Volterra integral equation of the second kind with a kernel containing a special function, namely, the degenerated hypergeometric Tricomi function. The limiting cases of the order of the fractional derivative in the term with the equation load are also investigated, and continuity in the order of the fractional derivative is shown. The solvability of the integral equation in the class of continuous functions is established depending on the nature of the load for small values of time.

\footnotetext{
${ }^{*}$ Corresponding author.

E-mail: isagyndyk@mail.ru
} 


\section{Basic definitions and some background}

Let us first recall some previously known concepts and results. The first one is the definition of the Riemann-Liouville fractional derivative. follows

Definition 1 ([1]). Let $f(t) \in L_{1}[a, b]$. Then, the Riemann-Liouville derivative of the order $\beta$ is defined as

$$
{ }_{r} D_{a, t}^{\beta} f(t)=\frac{1}{\Gamma(n-\beta)} \frac{d^{n}}{d t^{n}} \int_{a}^{t} \frac{f(\tau)}{(t-\tau)^{\beta-n+1}} d \tau, \quad \beta, a \in R, \quad n-1<\beta<n .
$$

When $a=0, \quad n=1$ we have:

$$
{ }_{r} D_{0, t}^{\beta} f(t)=\frac{1}{\Gamma(1-\beta)} \frac{d}{d t} \int_{0}^{t} \frac{f(t)}{(t-\tau)^{\beta}} d \tau
$$

From formula (1) it follows that

$$
{ }_{r} D_{a, t}^{0} f(t)=f(t), \quad{ }_{r} D_{a, t}^{n} f(t)=f^{(n)}(t), \quad n \in N .
$$

We also give definitions and some properties of special functions that arise in the study of boundary value problem posed in the work.

$\operatorname{erf} z=\frac{2}{\sqrt{\pi}} \int_{0}^{z} \exp \left(-\zeta^{2}\right) d \zeta$ is the integral of probabilities;

$\operatorname{erfc} z=\frac{2}{\sqrt{\pi}} \int_{z}^{\infty} \exp \left(-\zeta^{2}\right) d \zeta$ is the additional integral of probabilities.

Definition $2([17 ; 119])$ Linearly independent solutions of the equation

$$
\left[z D^{2}+(c-z) D-a\right] \omega(z)=0, \quad D=\frac{d}{d z}
$$

are functions $\Phi(a, c ; z)$ and $\Psi(a, c ; z)$, where $\Phi(a, c ; z)$ is the degenerate hypergeometric function:

$$
\Phi(a, c ; z)=1+\frac{a}{c} \frac{z}{1 !}+\frac{a(a+1)}{c(c+1)} \frac{z^{2}}{2 !}+\frac{a(a+1)(a+2)}{c(c+1)(c+2)} \frac{z^{3}}{3 !}+\ldots-
$$

and $\Psi(a, c ; z)$ is Tricomi degenerate hypergeometric function [18; 1072]:

$$
\Psi(a, c ; z)=\frac{\Gamma(1-c)}{\Gamma(a-c+1)} \Phi(a, c ; z)+\frac{\Gamma(c-1)}{\Gamma(a)} z^{1-c} \Phi(a-c+1,2-c ; z) .
$$

Tricomi degenerate hypergeometric function can be represented as an integral ([19; 365], formula 72.2 (7)):

$$
\Psi(a, c ; z)=\frac{1}{\Gamma(a)} \int_{0}^{\infty} \xi^{a-1}(1+\xi)^{c-a-1} e^{-z \xi} d \xi, \quad[\text { Re } a>0] .
$$

For large values $z$, an asymptotic formula holds ([17; 127], formula 4.7 (1)):

$$
\begin{gathered}
\Psi(a, c ; z) \sim z^{-a}{ }_{2} F_{0}\left(a, 1+a-c ;-\frac{1}{z}\right), \\
|z| \rightarrow \infty, \quad|\arg z| \leq \frac{3 \pi}{2}-\epsilon, \quad \epsilon>0,
\end{gathered}
$$

where ${ }_{2} F_{0}\left(a, 1+a-c ;-\frac{1}{z}\right)$ is a generalized hypergeometric series defined by the formula [17; 136]

$$
{ }_{p} F_{q}\left(a_{1}, a_{2}, \ldots, a_{p} ; b_{1}, b_{2}, \ldots, b_{q} ; z\right)=\sum_{k=0}^{\infty} \frac{\left(a_{1}\right)_{k}\left(a_{2}\right)_{k} \ldots\left(a_{p}\right)_{k}}{\left(b_{1}\right)_{k}\left(b_{2}\right)_{k} \ldots\left(b_{q}\right)_{k}} \frac{z^{k}}{k !},
$$

where

$$
(a)_{k}=\frac{\Gamma(a+k)}{\Gamma(a)}
$$

is the Pohammer symbol. 
Degenerate hypergeometric functions for some values of its arguments are related to a parabolic cylinder function (Weber function) $D_{\nu}(z)[17 ; 212]$

$$
\begin{gathered}
D_{\nu}(z)=2^{\frac{\nu-1}{2}} e^{-\frac{z^{2}}{4}} \Psi\left(\frac{1-\nu}{2}, \frac{3}{2} ; \frac{z^{2}}{2}\right)= \\
=2^{\frac{\nu}{2}} e^{-\frac{z^{2}}{4}}\left\{\frac{\sqrt{\pi}}{\Gamma\left(\frac{1-\nu}{2}\right)} \Phi\left(-\frac{\nu}{2} ; \frac{1}{2} ; \frac{z^{2}}{2}\right)-\frac{\sqrt{2 \pi} z}{\Gamma\left(-\frac{\nu}{2}\right)} \Phi\left(\frac{1-\nu}{2} ; \frac{3}{2} ; \frac{z^{2}}{2}\right)\right\} .
\end{gathered}
$$

Formula (6) is the definition of a parabolic cylinder function $D_{\nu}(z)$.

There is also a representation of the Tricomi degenerate hypergeometric function in terms of the Whittaker function $W_{\lambda, \mu}(z)([19 ; 264]$ formula 2.20$)$ :

$$
\Psi(a, b ; z)=z^{-\frac{b}{2}} e^{\frac{z}{2}} W_{\frac{b}{2}-a, \frac{b-1}{2}}(z) .
$$

For the function $W_{\lambda, \mu}(z) \quad z=0$ is a branch point, a $z=\infty$ is an essentially singular point [18; 1074]. Therefore, we will consider this function only for $|\arg z|<\pi$.

The natural development of fractional calculus is the theory of differential equations with fractional derivatives. At the first stage of the study, we will use the method of integral equations, in which the boundary value problem is reduced to solving the corresponding integral equation with further transformation of the kernel of the obtained equation. Such methods make it possible to formulate boundary value problems more compactly than differential equations, taking into account all the conditions of the problem.

The considered problem is reduced to an integral equation by inverting the differential part.

It's known $[20 ; 57]$ that in the domain $Q=\{(x, t) \mid x>0, \quad t>0\}$ the solution to the boundary value problem of heat conduction

$$
\begin{gathered}
u_{t}=a^{2} u_{x x}+F(x, t), \\
\left.u\right|_{t=0}=f(x),\left.\quad u\right|_{x=0}=g(x),
\end{gathered}
$$

is described by the formula

$$
\begin{aligned}
u(x, t)= & \int_{0}^{\infty} G(x, \xi, t) f(\xi) d \xi+\int_{0}^{t} H(x, t-\tau) g(\tau) d \tau+ \\
& +\int_{0}^{t} \int_{0}^{\infty} G(x, \xi, t-\tau) F(\xi, \tau) d \xi d \tau
\end{aligned}
$$

where

$$
\begin{gathered}
G(x, \xi, t)=\frac{1}{2 \sqrt{\pi a t}}\left\{\exp \left(-\frac{(x-\xi)^{2}}{4 a t}\right)-\exp \left(-\frac{(x+\xi)^{2}}{4 a t}\right)\right\}, \\
H(x, t)=\frac{1}{2 \sqrt{\pi a} t^{3 / 2}} \exp \left(-\frac{x^{2}}{4 a t}\right) .
\end{gathered}
$$

The Green function $G(x, \xi, t-\tau)$ satisfies the relation

$$
\int_{0}^{\infty} G(x, \xi, t-\tau) d \xi=\operatorname{erf}\left(\frac{x}{2 \sqrt{t-\tau}}\right) .
$$

2 Statement of the fractionally loaded boundary value problem of heat conduction

In the domain $Q=\{(x, t) \mid x>0, \quad t>0\}$ we consider the problem

$$
\begin{gathered}
u_{t}-u_{x x}+\left.\lambda\left\{{ }_{r} D_{0, t} u(x, t)\right\}\right|_{x=\gamma(t)}=f(x, t), \\
\left.u\right|_{t=0}=0,\left.u\right|_{x=0}=0,
\end{gathered}
$$

where $\lambda$ is a complex parameter, ${ }_{r} D_{0, t}^{\beta} u(x, t)$ is the Riemann- Liouville derivative (2) of an order $\beta, 0<\beta<1$, $\gamma(t)$ is a continuous increasing function, $\gamma(0)=0$.

The problem is studied in the class of continuous functions. 
3 Reducing the boundary value problem to an integral equation

Lemma 1. The boundary value problem (10)-(11) is equivalently reduced to a Volterra integral equation of the second kind with a kernel that contains a special function.

Proof. We invert the differential part of problem (10)-(11) by formula (8):

$$
\begin{gathered}
u(x, t)=-\left.\lambda \int_{0}^{t} \int_{0}^{\infty} G(x, \xi, t-\tau)\left\{\frac{1}{\Gamma(1-\beta)} \frac{d}{d \tau} \int_{0}^{\tau} \frac{u(\xi, \theta)}{(\tau-\theta)^{\beta}} d \theta\right\}\right|_{\xi=\gamma(\tau)} d \xi d \tau+ \\
+\int_{0}^{t} \int_{0}^{\infty} G(x, \xi, t-\tau) f(\xi, \tau) d \xi d \tau .
\end{gathered}
$$

Taking into account relation (9) and introducing the notation

$$
f_{1}(x, t)=\int_{0}^{t} \int_{0}^{\infty} G(x, \xi, t-\tau) f(\xi, \tau) d \tau
$$

we get the following representation of the solution to the problem (10)-(11):

$$
u(x, t)=-\lambda \int_{0}^{t} \operatorname{erf}\left(\frac{x}{2 \sqrt{t-\tau}}\right) \mu(\tau) d \tau+f_{1}(x, t),
$$

where

$$
\mu(t)=\left.{ }_{r} D_{0, t}^{\beta} u(\xi, t)\right|_{\xi=\gamma(t)}=\left.\left\{\frac{1}{\Gamma(1-\beta)} \frac{d}{d \tau} \int_{0}^{\tau} \frac{u(\xi, \theta)}{(\tau-\theta)^{\beta}} d \theta\right\}\right|_{\xi=\gamma(\tau)} .
$$

From (13) we take the derivative of the order $\beta$ with respect to the variables $t$ on both sides and put $x=\gamma(t)$. On the left side, we get the function $\mu(t)$. We also introduce the notation according to formula (14)

$$
f_{2}(t)=\left.{ }_{r} D_{0, t}^{\beta} f_{1}(x, t)\right|_{x=\gamma(t)}=\left.\frac{1}{\Gamma(1-\beta)} \frac{d}{d t} \int_{0}^{t} \frac{f_{1}(x, \tau)}{(t-\tau)^{\beta}} d \tau\right|_{x=\gamma(t)} .
$$

We first calculate the derivative:

$$
\begin{aligned}
& J(t, x, \beta)=\frac{d}{d t} \int_{0}^{t} \frac{1}{(t-\tau)^{\beta}}\left(\int_{0}^{\tau} \operatorname{erf}\left(\frac{x}{2 \sqrt{\tau-\theta}}\right) \mu(\theta) d \theta\right) d \tau=\left\|\begin{array}{c}
0 \leq \theta \leq \tau \\
0 \leq \tau \leq t
\end{array}\right\|= \\
& =\frac{d}{d t} \int_{0}^{t} \mu(\theta)\left(\int_{0}^{t} \frac{1}{(t-\tau)^{\beta}} \operatorname{erf}\left(\frac{x}{2 \sqrt{\tau-\theta}}\right) d \tau\right) d \theta=\frac{d}{d t} \int_{0}^{t} \mu(\theta) I(x, t, \theta, \beta) d \theta
\end{aligned}
$$

where

$$
I(x, t, \theta, \beta)=\int_{0}^{t} \frac{1}{(t-\tau)^{\beta}} \operatorname{erf}\left(\frac{x}{2 \sqrt{\tau-\theta}}\right) d \tau .
$$

We calculate $I(x, t, \theta, \beta)$.

$$
\begin{gathered}
I(x, t, \theta, \beta)=\int_{\theta}^{t} \frac{1}{(t-\tau)^{\beta}} \frac{2}{\sqrt{\pi}} \int_{0}^{\frac{x}{2 \sqrt{t-\theta}}} e^{-z^{2}} d z d \tau= \\
=\frac{2}{\sqrt{\pi}} \int_{0}^{\frac{x}{2 \sqrt{t-\theta}}} \int_{0}^{t} \frac{1}{(t-\tau)^{\beta}} e^{-z^{2}} d \tau d z+\frac{2}{\sqrt{\pi}} \int_{\frac{x}{2 \sqrt{t-\theta}}}^{+\infty} \int_{\theta}^{\theta+\frac{x^{2}}{4 z^{2}}} \frac{1}{(t-\tau)^{\beta}} e^{-z^{2}} d \tau d z= \\
=\frac{2}{\sqrt{\pi}(\beta-1)} \int_{0}^{\frac{x}{2 \sqrt{t-\theta}}} e^{-z^{2}}\left(0-(t-\theta)^{1-\beta}\right) d z+\frac{2}{\sqrt{\pi}(\beta-1)} \int_{\frac{x}{2 \sqrt{t-\theta}}}^{+\infty} e^{-z^{2} \times} \\
\times\left(\left(t-\theta-\frac{x^{2}}{4 z^{2}}\right)-(t-\theta)^{1-\beta}\right) d z=\frac{2(t-\theta)^{1-\beta}}{\sqrt{\pi}(1-\beta)} \int_{0}^{\frac{x}{2 \sqrt{t-\theta}}} e^{-z^{2}} d z+
\end{gathered}
$$




$$
\begin{gathered}
+\frac{2(t-\theta)^{1-\beta}}{\sqrt{\pi}(1-\beta)} \int_{\frac{x}{2 \sqrt{t-\theta}}}^{+\infty} e^{-z^{2}} d z+\frac{2}{\sqrt{\pi}(\beta-1)} \int_{\frac{x}{2 \sqrt{t-\theta}}}^{+\infty} e^{-z^{2}}\left(t-\theta-\frac{x^{2}}{4 z^{2}}\right)^{1-\beta} d z= \\
=\frac{(t-\theta)^{1-\beta}}{1-\beta}+\frac{2}{\sqrt{\pi}(\beta-1)} I_{1}(x, t, \theta, \beta),
\end{gathered}
$$

where

$$
\begin{gathered}
I_{1}(x, t, \theta, \beta)=\int_{\frac{x}{2 \sqrt{t-\theta}}}^{+\infty} e^{-z^{2}}\left(t-\theta-\frac{x^{2}}{4 z^{2}}\right)^{1-\beta} d z= \\
=(t-\theta)^{1-\beta} \int_{\frac{x}{2 \sqrt{t-\theta}}}^{+\infty} e^{-z^{2}} z^{2 \beta-2}\left(z^{2}-\left(\frac{x}{2 \sqrt{t-\theta}}\right)^{2}\right)^{1-\beta} d z=\left\|\xi=z^{2}\right\|= \\
=\frac{(t-\theta)^{1-\beta}}{2} \int_{\frac{x^{2}}{4(t-\theta)}}^{+\infty} e^{-\xi} \xi^{\beta-\frac{3}{2}}\left(\xi-\frac{x^{2}}{4(t-\theta)}\right)^{1-\beta} d \xi .
\end{gathered}
$$

To calculate the last integral, we use the formula 2.3 .4 (6) from [21; 261]. Then we obtain

$$
I_{1}(x, t, \theta, \beta)=\frac{(t-\theta)^{1-\beta}}{2} \Gamma(2-\beta) \frac{x}{2 \sqrt{t-\theta}} \exp \left(-\frac{x^{2}}{4(t-\theta)}\right) \Psi\left(2-\beta ; \frac{3}{2} ; \frac{x^{2}}{4(t-\theta)}\right),
$$

where $\Psi(a, c ; z)$ is Tricomi degenerate hypergeometric function [18; 1072].

Substituting (19) into (18), we get an expression for (17)

$$
I(x, t, \theta, \beta)=\frac{(t-\theta)^{1-\beta}}{1-\beta}-\frac{x \Gamma(1-\beta)(t-\theta)^{\frac{1}{2}-\beta}}{2 \sqrt{\pi}} \exp \left(-\frac{x^{2}}{4(t-\theta)}\right) \Psi\left(2-\beta ; \frac{3}{2} ; \frac{x^{2}}{4(t-\theta)}\right) .
$$

By virtue of the asymptotic formula (5) (as $t \rightarrow \tau$ ) and

$$
\lim _{t \rightarrow \tau}(t-\tau)^{\frac{1}{2}-\beta} \exp \left(-\frac{x^{2}}{4(t-\tau)}\right)=0
$$

after differentiation operation equality (16) can be rewritten as:

$$
\begin{aligned}
& J(t ; x ; \beta)=\int_{0}^{t} \mu(t) \frac{d}{d t}\left[\frac{(t-\tau)^{1-\beta}}{1-\beta}-\frac{x \Gamma(1-\beta)(t-\tau)^{\frac{1}{2}-\beta}}{2 \sqrt{\pi}} \times\right. \\
& \left.\quad \times \exp \left(-\frac{x^{2}}{4(t-\tau)}\right) \Psi\left(2-\beta ; \frac{3}{2} ; \frac{x^{2}}{4(t-\tau)}\right)\right] d \tau .
\end{aligned}
$$

Let us introduce the notation $z=\frac{x^{2}}{4(t-\tau)} \Rightarrow t-\tau=\frac{x^{2}}{4 z}$.

Then

$$
(t-\tau)^{\frac{1}{2}-\beta} \exp \left(-\frac{x^{2}}{4(t-\tau)}\right) \Psi\left(2-\beta ; \frac{3}{2} ; \frac{x^{2}}{4(t-\tau)}\right)=\frac{x^{1-2 \beta}}{2^{1-2 \beta}} z^{\beta-\frac{1}{2}} \exp (-z) \Psi\left(2-\beta ; \frac{3}{2} ; z\right) .
$$

To calculate the derivative in the second term of equality (20), we use formula (41) from [19; 368] when $n=1$ :

$$
\begin{gathered}
\frac{d}{d t}\left((t-\tau)^{\frac{1}{2}-\beta} \exp \left(-\frac{x^{2}}{4(t-\tau)}\right) \Psi\left(2-\beta ; \frac{3}{2} ; \frac{x^{2}}{4(t-\tau)}\right)\right)= \\
=\frac{x^{1-2 \beta}}{2^{1-2 \beta}} \frac{d}{d z}\left(z^{\beta-\frac{1}{2}} \exp (-z) \Psi\left(2-\beta ; \frac{3}{2} ; z\right)\right) \frac{d z}{d t}= \\
=-\frac{x^{1-2 \beta}}{2^{1-2 \beta}} z^{\beta-\frac{3}{2}} \exp (-z) \Psi\left(1-\beta ; \frac{3}{2} ; z\right)\left(-\frac{x}{4(t-\tau)^{2}}\right)= \\
=\frac{1}{(t-\tau)^{\beta+\frac{1}{2}}} \exp \left(-\frac{x^{2}}{4(t-\tau)}\right) \Psi\left(1-\beta ; \frac{3}{2} ; \frac{x^{2}}{4(t-\tau)}\right) .
\end{gathered}
$$

Mathematics series. № 1(101)/2021 
Then expression (20) can be rewritten as:

$$
J(t ; x ; \beta)=\int_{0}^{t} \mu(\tau)\left[\frac{1}{(t-\tau)^{\beta}}-\frac{x \Gamma(1-\beta)}{2 \sqrt{\pi}(t-\tau)^{\beta+\frac{1}{2}}} \exp \left(-\frac{x^{2}}{4(t-\tau)}\right) \Psi\left(1-\beta ; \frac{3}{2} ; \frac{x^{2}}{4(t-\tau)}\right)\right] d \tau
$$

Now from (13) after taking the fractional derivative of order $\beta$ and substituting $x=\gamma(t)$ taking into account the notation (14) and (21) we obtain the integral equation:

$$
\begin{gathered}
\mu(t)+\frac{\lambda}{\Gamma(1-\beta)} \int_{0}^{t} \frac{\mu(\tau)}{(t-\tau)^{\beta}} d \tau-\frac{\lambda}{2 \sqrt{\pi}} \int_{0}^{t} \frac{\gamma(t)}{(t-\tau)^{\beta+\frac{1}{2}}} \exp \left(-\frac{\gamma^{2}(t)}{4(t-\tau)}\right) \times \\
\times \Psi\left(1-\beta, \frac{3}{2} ; \frac{\gamma^{2}(t)}{4(t-\tau)}\right) \mu(\tau) d \tau=f_{2}(t) .
\end{gathered}
$$
kind:

So, the boundary value problem (10)-(11) has been reduced to the Volterra integral equation of the second

$$
\mu(t)+\lambda \int_{0}^{t} K_{\beta}(t, \tau) \mu(\tau) d \tau=f_{2}(t)
$$

with the right-hand side $f_{2}(t)$, defined by formula (15), and the kernel

$$
K_{\beta}(t, \tau)=\frac{1}{\Gamma(1-\beta)(t-\tau)^{\beta}}-\frac{\gamma(t)}{2 \sqrt{\pi}(t-\tau)^{\beta+\frac{1}{2}}} \exp \left(-\frac{\gamma^{2}(t)}{4(t-\tau)}\right) \Psi\left(1-\beta, \frac{3}{2} ; \frac{\gamma^{2}(t)}{4(t-\tau)}\right)
$$

where $\Psi(a, b, y)$ is the Tricomi degenerate hypergeometric function that can be represented as an integral (4).

\section{Continuity in the order of the derivative in the loaded term of the problem}

Lemma 2. For boundary value problem (10)-(11) there is continuity in the order $\beta$ of the derivative in the loaded term of equation (10).

Proof. We consider the limiting cases for the fractional derivative order of the term with the load in the equation (10).

I. $\quad \beta=0$. Then from (2) and (3) we have

$$
\left.D_{0, t}^{0} u(x, t)\right|_{x=\gamma(t)}=\left.u(x, t)\right|_{x=\gamma(t)}=u(\gamma(t), t) .
$$

From (10)-(11) we get a boundary value problem when $\beta=0$ :

$$
\left\{\begin{array}{c}
u_{t}-u_{x x}+\lambda \mu(t)=f(x, t) \\
u(x, 0)=0 ; \quad u(0, t)=0
\end{array},\right.
$$

where $\mu(t)=u(\gamma(t), t)$.

We write down its solution inverting the differential part by formula (8):

$$
u(x, t)=-\lambda \int_{0}^{t} \operatorname{erf}\left(\frac{x}{2 \sqrt{t-\tau}}\right) \mu(\tau) d \tau+f_{1}(x, t),
$$

where

$$
f_{1}(x, t)=\int_{0}^{t} \int_{0}^{\infty} G(x, \xi, t-\tau) f(\xi, \tau) d \xi d \tau .
$$

When $x=\gamma(t)$ taking into account the notation $u(\gamma(t), t)=\mu(t)$ from (24) we obtain the Volterra integral equation of the second kind:

$$
\mu(t)+\lambda \int_{0}^{t} \operatorname{erf}\left(\frac{\gamma(t)}{2 \sqrt{t-\tau}}\right) \mu(\tau) d \tau=f_{1}(t)
$$

where $f_{2}(t)=f_{1}(\gamma(t), t)$.

Now we find $\lim _{\beta \rightarrow 0+0}$ from (23). 
The function under the limit sign is definite and continuous for $\beta=0$, therefore, we can make the passage to the limit taking into account formula (4) and formula 2.3.4 (5) [21; 260]:

$$
\begin{aligned}
\lim _{\beta \rightarrow 0+0} K_{\beta}(t, \tau) & =1-\frac{\gamma(t)}{2 \sqrt{\pi} \sqrt{t-\tau}} \exp \left(-\frac{\gamma^{2}(t)}{4(t-\tau)}\right) \Psi\left(1 ; \frac{3}{2} ; \frac{\gamma^{2}(t)}{4(t-\tau)}\right)= \\
=1-\frac{\gamma(t)}{2 \sqrt{\pi} \sqrt{t-\tau}} & \exp \left(-\frac{\gamma^{2}(t)}{4(t-\tau)}\right) \int_{0}^{\infty}(1+\xi)^{-\frac{1}{2}} \exp \left(-\frac{\gamma^{2}(t)}{4(t-\tau)} \xi\right) d \xi= \\
= & 1-\operatorname{erfc}\left(\frac{\gamma(t)}{2 \sqrt{t-\tau}}\right)=\operatorname{erf}\left(\frac{\gamma(t)}{2 \sqrt{t-\tau}}\right) .
\end{aligned}
$$

So, $\lim _{\beta \rightarrow 0+0} K_{\beta}(t, \tau)=\operatorname{erf}\left(\frac{\gamma(t)}{2 \sqrt{t-\tau}}\right)$. Then equation (22) coincides with equation (25) for $\beta=0$.

II. $\beta=1$. Then from (2) and (3) we have

$$
\left.D_{0, t}^{1} u(x, t)\right|_{x=\gamma(t)}=\left.\frac{d u(x, t)}{d t}\right|_{x=\gamma(\tau)}=\left.u_{t}(x, t)\right|_{x=\gamma(t)} .
$$

From (10)-(11) we get a boundary value problem when $\beta=1$ :

$$
\left\{\begin{array}{c}
u_{t}-u_{x x}+\lambda \mu(t)=f(x, t) \\
u(x, 0)=0 ; \quad u(0, t)=0
\end{array}\right.
$$

where $\mu(t)=\left.u_{t}(x, t)\right|_{x=\gamma(t)}$.

We write down its solution inverting the differential part by formula (8):

$$
u(x, t)=-\lambda \int_{0}^{t} \operatorname{erf}\left(\frac{x}{2 \sqrt{t-\tau}}\right) \mu(\tau) d \tau+f_{1}(x, t),
$$

where the function $f_{1}(x, t)$ is defined by formula (12).

We calculate the derivative with respect to $t$ of $(26)$ :

$$
u_{t}(x, t)=-\lambda\left\{\mu(t)+\int_{0}^{t} \frac{2}{\sqrt{\pi}} \exp \left(-\frac{x^{2}}{4(t-\tau)}\right)\left(-\frac{x}{4(t-\tau)^{3 / 2}}\right) \mu(\tau) d \tau\right\}+f_{1 t}(x, t) .
$$

Substituting $x=\gamma(t)$ and taking into account the notation $\left.u_{t}(x, t)\right|_{x=\gamma(t)}=\mu(t)$ we obtain the Volterra integral equation of the second kind:

$$
\mu(t)-\frac{\lambda}{1+\lambda} \int_{0}^{t} \frac{\gamma(t)}{2 \sqrt{\pi}(t-\tau)^{3 / 2}} \exp \left(-\frac{\gamma^{2}(t)}{4(t-\tau)}\right) \mu(\tau) d \tau=f_{2}(t)
$$

where $f_{2}(t)=\frac{1}{1+\lambda} f_{1 t}(\gamma(t), t)$.

Taking into account formula (4) and the well-known relation $\lim _{\alpha \rightarrow 0} \Gamma(\alpha)=\infty$ when taking the limit at $\beta \rightarrow 1-0$ from (23) we get:

$$
\begin{gathered}
\lim _{\beta \rightarrow 1-0} K_{\beta}(t, \tau)=-\frac{\gamma(t)}{2 \sqrt{\pi(t-\tau)^{3}}} \exp \left(-\frac{\gamma^{2}(t)}{4(t-\tau)}\right) \times \\
\times \lim _{\beta \rightarrow 1-0} \frac{1}{\Gamma(1-\beta)} \int_{0}^{\infty} \xi^{-\beta}(1+\xi)^{\beta-\frac{1}{2}} \exp \left(-\frac{\gamma^{2}(t)}{4(t-\tau)} \xi\right) d \xi .
\end{gathered}
$$

To calculate the last integral, we use the formula (12) from [21; 262].

Then the limit relation (28) can be rewritten as:

$$
\begin{gathered}
\lim _{\beta \rightarrow 1-0} K_{\beta}(t, \tau)=-\frac{1}{\sqrt{\pi}(t-\tau)} \exp \left(-\frac{\gamma^{2}(t)}{8(t-\tau)}\right) \lim _{\beta \rightarrow 1-0} \frac{2^{1 / 2-\beta} \Gamma(1-\beta)}{\Gamma(1-\beta)} D_{2 \beta-1}\left(\frac{\gamma(t)}{\sqrt{2(t-\tau)}}\right)= \\
=-\frac{2^{-\frac{1}{2}}}{\sqrt{\pi}(t-\tau)} \exp \left(-\frac{\gamma^{2}(t)}{8(t-\tau)}\right) D_{1}\left(\frac{\gamma(t)}{\sqrt{2(t-\tau)}}\right)=-\frac{\gamma(t)}{2 \sqrt{\pi}(t-\tau)^{3 / 2}} \exp \left(-\frac{\gamma^{2}(t)}{4(t-\tau)}\right) .
\end{gathered}
$$


Here, for the parabolic cylinder function $D_{2 \beta-1}(z)$, we used formula 9.251 from $[18 ; 1080]$ when $\beta=1$ :

$$
D_{1}(z)=-e^{\frac{z^{2}}{4}} \frac{d}{d z}\left(e^{-\frac{z^{2}}{2}}\right)=z e^{-\frac{z^{2}}{4}} .
$$

So for equation $(22)$

$$
\lim _{\beta \rightarrow 1} K_{\beta}(t, \tau)=-\frac{\gamma(t)}{2 \sqrt{\pi}(t-\tau)^{3 / 2}} \exp \left(-\frac{\gamma^{2}(t)}{4(t-\tau)}\right) .
$$

The obtained result coincides with the kernel of integral equation (27).

Lemma 2 is completely proved.

5 Connection of the singularities of the integral equation kernel with the fractional derivative order in the problem loaded term and with the load behavior. Main result

To establish the main result of the paper we investigate kernel (23) of integral equation (22), which has singularities for $\tau=t$ and $t=0$.

Direct investigation of kernel (23) is difficult, since the kernel contains the degenerate hypergeometric Tricomi function. Therefore we find

$$
\lim _{t \rightarrow 0+0} \int_{0}^{t} K_{\beta}(t, \tau) d \tau \text {. }
$$

Theorem. Integral equation (22) with kernel (23) for $0 \leq \beta<1$ and with $\gamma(t) \sim t^{\omega}$ in the neighborhood of $t=0$ is uniquely solvable in the class of continuous functions for any continuous right-hand side $f_{2}(t)$ defined by formula (15), if $\frac{1}{2} \leq \omega<1-2 \beta$ or $0 \leq \omega<\frac{1}{2}, \quad 0 \leq \beta \leq 1$.

Proof. We have

$$
\begin{gathered}
\int_{0}^{t} K_{\beta}(t, \tau) d \tau=\frac{1}{\Gamma(1-\beta)} \int_{0}^{t} \frac{d \tau}{(t-\tau)^{\beta}}- \\
-\frac{\gamma(t)}{2 \sqrt{\pi}} \int_{0}^{t} \frac{1}{(t-\tau)^{\beta+\frac{1}{2}}} \exp \left(-\frac{\gamma^{2}(t)}{4(t-\tau)}\right) \Psi\left(1-\beta ; \frac{3}{2} ; \frac{\gamma^{2}(t)}{4(t-\tau)}\right) d \tau
\end{gathered}
$$

and when $0 \leq \beta<1$ :

$$
\int_{0}^{t} \frac{d \tau}{(t-\tau)^{\beta}}=\frac{1}{1-\beta} t^{1-\beta}
$$

To calculate the integral in the 2 nd term of expression (29), we use the representation of the Tricomi function in terms of the Whittaker function by formula (7):

$$
\Psi\left(1-\beta ; \frac{3}{2} ; \frac{\gamma^{2}(t)}{4(t-\tau)}\right)=\left(\frac{\gamma^{2}(t)}{4(t-\tau)}\right)^{-\frac{3}{4}} \exp \left(\frac{\gamma^{2}(t)}{8(t-\tau)}\right) W_{\beta-\frac{1}{4} ; \frac{1}{4}}\left(\frac{\gamma^{2}(t)}{4(t-\tau)}\right)
$$

Then the integral in the 2nd term of the expression (29) takes the form

$$
\begin{gathered}
I(t, \beta)=\int_{0}^{t} \frac{1}{(t-\tau)^{\beta+\frac{1}{2}}} \exp \left(-\frac{\gamma^{2}(t)}{4(t-\tau)}\right) \Psi\left(1-\beta ; \frac{3}{2} ; \frac{\gamma^{2}(t)}{4(t-\tau)}\right) d \tau= \\
=\frac{2^{3 / 2}}{(\gamma(t))^{3 / 2}} \int_{0}^{t}(t-\tau)^{\frac{1}{4}-\beta} \exp \left(-\frac{\gamma^{2}(t)}{8(t-\tau)}\right) W_{\beta-\frac{1}{4} ; \frac{1}{4}}\left(\frac{\gamma^{2}(t)}{4(t-\tau)}\right) d \tau= \\
=\frac{2^{2 \beta-1}}{(\gamma(t))^{2 \beta-1}} \int_{\frac{\gamma^{2}(t)}{4 t}}^{+\infty} z^{\beta-\frac{9}{4}} e^{-\frac{z}{2}} W_{\beta-\frac{1}{4} ; \frac{1}{4}}(z) d z=\frac{2^{3 / 2} t^{\frac{5}{4}-\beta}}{(\gamma(t))^{3 / 2}} \exp \left(-\frac{\gamma^{2}(t)}{8 t}\right) W_{\beta-\frac{5}{4} ; \frac{1}{4}}\left(\frac{\gamma^{2}(t)}{4 t}\right) .
\end{gathered}
$$

In the calculation, we have introduced the replacement $z=\frac{\gamma^{2}(t)}{4(t-\tau)}$ and used formula 2.19.5 (13) from [19; $217]$.

Let $\gamma(t) \sim t^{\omega}$ when $t \rightarrow 0+0$. Then (31) can be rewritten as (in the neighborhood of the point $t=0$ ):

$$
I(t ; \beta)=2^{\frac{3}{2}} t^{\frac{5}{4}-\beta-\frac{3}{2} \omega} \exp \left(-\frac{1}{8} t^{2 \omega-1}\right) W_{\beta-\frac{5}{4} ; \frac{1}{4}}\left(\frac{1}{4} t^{2 \omega-1}\right) .
$$


For Whittaker function, we use formula 7.2.2 (5) from [19; 366]:

$$
W_{\beta-\frac{5}{4} ; \frac{1}{4}}\left(\frac{1}{4} t^{2 \omega-1}\right)=2^{-\frac{3}{2}} t^{\frac{1}{2} \omega-\frac{3}{4}} \exp \left(-\frac{1}{8} t^{2 \omega-1}\right) \Psi\left(2-\beta ; \frac{3}{2} ; \frac{1}{4} t^{2 \omega-1}\right) .
$$

Then

$$
I(t ; \beta)=t^{\frac{1}{2}-\beta-\omega} \exp \left(-\frac{1}{4} t^{2 \omega-1}\right) \Psi\left(2-\beta ; \frac{3}{2} ; \frac{1}{4} t^{2 \omega-1}\right) .
$$

For Tricomi function $\Psi(a, b, z)$, we use representation (4) and then we apply formula (12) from [21; 262]:

$$
\begin{gathered}
I(t ; \beta)=\frac{t^{\frac{1}{2}-\beta-\omega}}{\Gamma(2-\beta)} \exp \left(-\frac{1}{4} t^{2 \omega-1}\right) \int_{0}^{\infty} \xi^{1-\beta}(1+\xi)^{\beta-\frac{3}{2}} \exp \left(-\frac{1}{4} t^{2 \omega-1} \xi\right) d \xi= \\
=2^{\frac{5}{2}-\beta} t^{1-2 \beta-2 \omega} \exp \left(-\frac{1}{8} t^{2 \omega-1}\right) D_{2 \beta-3}\left(\frac{1}{\sqrt{2}} t^{\omega-\frac{1}{2}}\right) .
\end{gathered}
$$

So, substituting (30) and (32) into (29), we get $0 \leq \beta<1$ when $\quad t \rightarrow 0$ :

$$
\int_{0}^{t} K_{\beta}(t, \tau) d \tau=\frac{t^{1-\beta}}{\Gamma(2-\beta)}-\frac{2^{\frac{3}{2}-\beta}}{\sqrt{\pi}} t^{1-2 \beta-\omega} \exp \left(-\frac{1}{8} t^{2 \omega-1}\right) D_{2 \beta-3}\left(\frac{1}{\sqrt{2}} t^{\omega-\frac{1}{2}}\right) .
$$

Cases are possible (when $0 \leq \beta<1$ ):

1) $2 \omega-1>0$. If $0 \leq \beta<1$, then $-1-2 \beta-\omega<\frac{1}{2}$.

We first calculate $D_{2 \beta-3}(0)$ using formula 8.3 (1) from [22; 125] and formula 194 (3) from [18; 299].

$$
D_{2 \beta-3}(0)=\frac{2^{\beta-\frac{3}{2}}}{\Gamma\left(\frac{3}{2}-\beta\right)} \int_{0}^{\infty} t^{\frac{1}{2}-\beta}(1+t)^{\beta-2} d t=\frac{2^{\beta-\frac{3}{2}}}{\Gamma\left(\frac{3}{2}-\beta\right)} B\left(\frac{3}{2}-\beta ; \frac{1}{2}\right)=\frac{2^{\beta-\frac{3}{2}} \sqrt{\pi}}{\Gamma(2-\beta)} .
$$

Then from (33) when $\omega>\frac{1}{2}$ and $1-2 \beta-\omega \geq 0 \quad(0 \leq \beta<1)$ we have:

$$
\lim _{t \rightarrow 0} \int_{0}^{t} K_{\beta}(t, \tau) d \tau= \begin{cases}0 ; & \text { if } 1-2 \beta-\omega>0, \quad \omega>\frac{1}{2}, \quad 0 \leq \beta<1 ; \\ -\frac{1}{\Gamma(2-\beta)} ; & \text { if } 1-2 \beta-\omega=0, \quad \omega>\frac{1}{2}, \quad 0 \leq \beta<1 \\ \infty ; & \text { if } 1-2 \beta-\omega<0, \quad \omega>\frac{1}{2}, \quad 0 \leq \beta<1 .\end{cases}
$$

We consider the case $\beta=1$. It was shown above that

$$
K_{1}(t, \tau)=-\frac{\gamma(t)}{2 \sqrt{\pi}(t-\tau)^{3 / 2}} \exp \left(-\frac{\gamma^{2}(t)}{4(t-\tau)}\right) .
$$

If $\gamma(t) \sim t^{\omega}$ at $t \rightarrow 0+0$ then

$$
\int_{0}^{t} K_{1}(t, \tau) d \tau=-\int_{0}^{t} \frac{t^{\omega}}{2 \sqrt{\pi}(t-\tau)^{3 / 2}} \exp \left(-\frac{t^{2 \omega}}{4(t-\tau)}\right) d \tau .
$$

After introducing the replacement

$$
\begin{aligned}
& z=\sqrt{\frac{t^{2 \omega}}{4(t-\tau)}} ; \quad t-\tau=\frac{t^{2 \omega}}{4 z^{2}} ; \quad d \tau=\frac{t^{2 \omega}}{2 z^{3}} d z \\
& \tau=0 \Rightarrow z=\frac{1}{2} t^{\frac{2 \omega-1}{2}}, \quad \tau=t \Rightarrow z \rightarrow+\infty
\end{aligned}
$$

integral (35) takes the form:

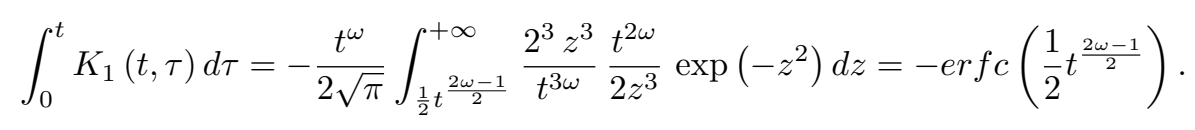

Then

$$
\lim _{t \rightarrow 0+0} \int_{0}^{t} K_{1}(t, \tau) d \tau= \begin{cases}-1 ; & \text { if } \quad \omega>\frac{1}{2} \\ 0 ; & \text { if } \quad 0 \leq \omega<\frac{1}{2} .\end{cases}
$$


By the condition stated, the function $\gamma(t)$ increases in the domain $\bar{Q}$ and $\gamma(0)=0$. Therefore, the case $\omega<0$ is not considered.

Consider the case $0<\omega<\frac{1}{2}$ and $0 \leq \beta<1$. Then in (33) argument of the parabolic cylinder function $\rightarrow+\infty$ if $t \rightarrow 0+0$. Since there is an asymptotic expansion of the function (see formula 9.246 (1) [18; 1079]) we obtain

$$
\lim _{t \rightarrow 0+0} \int_{0}^{t} K_{\beta}(t, \tau) d \tau=\lim _{t \rightarrow 0+0} \frac{t^{1-\beta}}{\Gamma(2-\beta)}-\frac{1}{\sqrt{\pi}} \lim _{t \rightarrow 0+0} t^{\frac{3}{2}-2 \omega(1-\beta)-3 \beta} \exp \left(-\frac{1}{4 t^{\frac{1}{2}-\omega}}\right)=0 .
$$

It remains to investigate the case $\omega=\frac{1}{2}$ for different values $\beta$.

Let be $0 \leq \beta<1$. For $x=\gamma(t)=\sqrt{t}$ equation (22) has the kernel:

$$
K_{\beta}(t, \tau)=\frac{1}{\Gamma(1-\beta)(t-\tau)^{\beta}}-\frac{\sqrt{t}}{2 \sqrt{\pi}(t-\tau)^{\beta+\frac{1}{2}}} \exp \left(-\frac{t}{4(t-\tau)}\right) \Psi\left(1-\beta ; \frac{3}{2} ; \frac{t}{4(t-\tau)}\right)
$$

and the right side according to formula (15) when $x=\sqrt{t}$. Kernel (38) has singularities at $\tau=t$ and $t=0$. We find

$$
\int_{0}^{t} K_{\beta}(t, \tau) d \tau=\frac{t^{1-\beta}}{\Gamma(2-\beta)}-\frac{\sqrt{t}}{2 \sqrt{\pi}} \int_{0}^{t} \frac{1}{(t-\tau)^{\beta+\frac{1}{2}}} \exp \left(-\frac{t}{4(t-\tau)}\right) \Psi\left(1-\beta ; \frac{3}{2} ; \frac{t}{4(t-\tau)}\right) d \tau .
$$

Repeating the above calculations, we get a formula similar to (33), when $0 \leq \beta<1$

$$
\int_{0}^{t} K_{\beta}(t, \tau) d \tau=\frac{t^{1-\beta}}{\Gamma(2-\beta)}-\frac{2^{\frac{1}{2}-\beta}}{\sqrt{\pi}} t^{\frac{1}{2}-2 \beta} \exp \left(-\frac{1}{8}\right) D_{2 \beta-3}\left(\frac{1}{\sqrt{2}}\right) .
$$

We calculate $D_{2 \beta-3}\left(\frac{1}{\sqrt{2}}\right)$ using formula 9.241 (1) from [18; 1078].

$$
D_{2 \beta-3}\left(\frac{1}{\sqrt{2}}\right)=\frac{1}{\sqrt{\pi}} 2^{2 \beta-\frac{1}{2}} e^{-\frac{\pi}{2}(2 \beta-1) i} e^{\frac{1}{8}} \int_{-\infty}^{+\infty} x^{2 \beta-1} e^{-2 x^{2}+\frac{x}{\sqrt{2}} i} d x .
$$

To calculate the integral

$$
J(\beta)=\int_{-\infty}^{+\infty} x^{2 \beta-1} e^{-2 x^{2}+\frac{x}{\sqrt{2}} i} d x
$$

we use the formula $3.462(3)$ from $[18 ; 352]$

$$
J(\beta)=\frac{1}{i^{2 \beta-1}} \int_{-\infty}^{+\infty}(i x)^{2 \beta-1} e^{-2 x^{2}-i \frac{x}{\sqrt{2}}} d x=\frac{2^{\frac{1}{2}-2 \beta}}{i^{2 \beta-1}} \sqrt{\pi} e^{-\frac{1}{32}} D_{2 \beta-3}\left(\frac{1}{2 \sqrt{2}}\right) .
$$

Then

$$
D_{2 \beta-3}\left(\frac{1}{\sqrt{2}}\right)=\frac{1}{i^{2 \beta-2}} \sin \left(\frac{\pi}{2}-\pi \beta\right) D_{2 \beta-3}\left(\frac{1}{2 \sqrt{2}}\right) .
$$

Note that the argument of the parabolic cylinder function decreases exponentially with the denominator $\frac{1}{2}$, remaining positive. It was previously calculated that

$$
D_{2 \beta-3}(0)=\frac{2^{\beta-\frac{3}{2}} \sqrt{\pi}}{\Gamma(2-\beta)}
$$

So we obtain that $D_{2 \beta-3}\left(\frac{1}{\sqrt{2}}\right)$ is a finite constant depending on $\beta$.

Then from (39) we have (when $0 \leq \beta<1$ and $\omega=\frac{1}{2}$ ):

$$
\lim _{t \rightarrow 0+0} \int_{0}^{t} K_{1}(t, \tau) d \tau= \begin{cases}0 ; & \text { if } \quad 0 \leq \beta<\frac{1}{4} \\ \text { const } \neq 0 ; & \text { if } \beta=\frac{1}{4} \\ \infty ; & \text { if } \frac{1}{4}<\beta<1\end{cases}
$$


Now consider the case for $\gamma(t)=\sqrt{t}$ and $\beta=1$ :

$$
\begin{aligned}
\int_{0}^{t} K_{1}(t, \tau) d \tau & =-\frac{\sqrt{t}}{2 \sqrt{\pi}} \int_{0}^{t} \frac{d \tau}{(t-\tau)^{\frac{3}{2}}} \exp \left(-\frac{t}{4(t-\tau)}\right) d \tau=\left\|\begin{array}{c}
z=\sqrt{t-\tau}, \tau=t-z^{2}, \quad \|= \\
d \tau=-2 z d z
\end{array}\right\| \\
& =-\frac{\sqrt{t}}{\sqrt{\pi}} \int_{0}^{\sqrt{t}} \frac{1}{z^{2}} e^{-\frac{t}{4 z^{2}}} d z=-\frac{2}{\sqrt{\pi}} \int_{\frac{1}{2}}^{+\infty} e^{-\xi^{2}} d \xi=-\operatorname{erfc}\left(\frac{1}{2}\right) .
\end{aligned}
$$

Then

$$
\lim _{t \rightarrow 0} \int_{0}^{t} K_{1}(t, \tau) d \tau=-\operatorname{erfc}\left(\frac{1}{2}\right) \neq 0
$$

if $\gamma(t)=\sqrt{t}$ and $\beta=1$.

Summarizing results (34)-(41), we get the main result. The theorem is completely proved.

\section{Conclusions}

Under the conditions of the theorem, kernel (23) of the integral equation has a weak singularity. Therefore, the method of successive approximations can be used to find a unique solution to the equation (22) in the class of continuous functions. And the corresponding boundary value problems are well-posed in natural classes of functions, i.e. loaded term is a weak perturbation.

If $\omega \geq \frac{1}{2}$ and $\omega \geq 1-2 \beta$ when $0 \leq \beta \leq 1$ for $\gamma(t) \sim t^{\omega}$ at $t \rightarrow 0+0$ integral equation (22) is not solvable by the method of successive approximations. It can be shown that the corresponding homogeneous equation for some values of the parameter $\lambda$ will have nonzero solutions. If the uniqueness of the solution to the first boundary value problem is violated, then in this case the load can be interpreted as a strong perturbation. So, the existence and uniqueness of solutions to the integral equation depends on the order of the fractional derivative in the loaded term.

\section{Acknowledgments}

This research was funded by the Science Committee of the Ministry of Education and Science of the Republic of Kazakhstan (Grant No. AP08955795, 2020-2022.)

\section{References}

1 Samko, S. G., Kilbas, A. A., \& Marichev, O. I. (1993). Fractional Integrals and Derivatives. Theory and Application. Gordon and Breach: New York, 1006 p.

2 Le Mehaute, A., Tenreiro Machado, J. A., Trigeassou, J. C., \& Sabatier, J. (2005). Fractional Differentiation and its Applications. Bordeaux Univ: Bordeaux.

3 Podlubny, I. (2002). Geometric and physical interpretation of fractional integration and fractional differentiation. Fract. Calculus Appl. Anal., 5, 367-386. an: 1042.26003.

4 Heymans, N., \& Podlubny, I. (2006). Physical interpretation of initial conditions for fractional differential equations with Riemann-Liouville fractional derivatives. Rheologica Acta, 45(5), 765-772. DOI: 10.1007 /S00397-005-0043-5.

5 Feng, M., Zhang, X., \& Ge, W. (2011). New existence results for higher-order nonlinear fractional differential equations with integral boundary conditions. Bound. Value Probl. Art., 720702, 20. DOI: $10.1155 / 2011 / 720702$.

6 Cao Labora, Daniel, Rodriguez-Lopez, Rosana, \& Belmekki, Mohammed. (2020). Existence of solutions to nonlocal boundary value problems for fractional differential equations with impulses. Electronic Journal of Differential Equations, 15, 16.

7 Yusuf, A., Qureshi, S., Inc, M., Aliyu, A.I., Baleanu, D., \& Shaikh, A.A. (2018). Two-strain epidemic model involving fractional derivative with Mittag-Leffler kernel. Chaos: An Interdisciplinary Journal of Nonlinear Science, 28(12), 123121. DOI: 10.1063/1.5074084.

8 Nakhushev, A. M. (1983). Loaded equations and their applications. Diff. equations, 19(1), 86-94. 
9 Amangaliyeva, M. M., Akhmanova, D. M., Dzhenaliev, M. T., \& Ramazanov, M. I. (2011). Boundary value problems for a spectrally loaded heat operator with load line approaching the time axis at zero or infinity. Differential Equations, 47(2), 231-243. DOI: 10.1134/S0012266111020091

10 Dzhenaliev, M. T., \& Ramazanov, M. I. (2006). On the boundary value problem for the spectrally loaded heat conduction operator. Siberian Mathematical Journal, 47(3), 433-451. DOI: 10.1007/s11202-0060056-Z

11 Dzhenaliev, M. T., \& Ramazanov, M. I. (2007). On a boundary value problem for a spectrally loaded heat operator: I Differential Equations, 43(4), 513-524. DOI: 10.1134/S0012266107040106

12 Dzhenaliev, M. T., \& Ramazanov, M. I. (2007). On a boundary value problem for a spectrally loaded heat operator: II Differential Equations, 43(6), 806-812. DOI: 10.1134/S0012266107060079

13 Attayev, A. Kh., Iskakov, S. A., Karshigina, G. Zh., \& Ramazanov, M. I. (2014). The first boundary problem for heat conduction equation with a load of fractional order. I. Bulletin of the Karaganda University-mathematics, 4(76), 11-16.

14 Iskakov, S. A., Ramazanov, M. I., \& Ivanov, I. A. (2015). The first boundary problem for heat conduction equation with a load of fractional order. II. Bulletin of the Karaganda University-mathematics, 2(78), 25-30.

15 Kosmakova, M. T., \& Kasymova, L. Zh. (2019). To solving the heat equation with fractional load. Journal of Mathematics, Mechanics and Computer Science, 104(4), 50-62. DOI: 10.26577/JMMCS-2019-4-m6.

Ramazanov, M. I., Kosmakova, M. T., \& Kasymova, L. Zh. (2020). On a Problem of Heat Equation with Fractional Load. Lobachevskii Journal of Mathematics, 41(9), 1873-1885.

DOI: 10.1134/S199508022009022X

16 Luke, Yudell L. (1969). The special functions and their approximations. Academic Press, Inc.: London.

17 Gradshteyn, I.S., \& Ryzhik, I.M. (2007). Table of Integrals, Series, and Products. AP: New York. USA. 7 edition.

18 Prudnikov, A. P., Brychkov, Yu. A., \& Marichev, O. I. (1989). Integrals and Series: More Special Functions. Vol. 3. Gordon and Breach: New York-London.

19 Polyanin, A.D. (2002). Handbook of Linear Partial Differential Equations for Engineers and Scientists. Chapman and Hall/CRC: New York-London.

20 Prudnikov, A. P., Brychkov, Yu. A., \& Marichev, O. I. (1998). Integrals and Series: Elementary Functions. (N.M.Queen, Trans) CRC: New York, USA.

21 Bateman, H., \& Erderlyi, A. (1953). Higher transcendental functions. McGraw-Hill: New York.

\author{
М.Т. Космакова, С.А. Искаков, Л.Ж. Касымова
}

\title{
Жойылатын облыстағы жылуөткізгіштіктің екіөлшемді шекаралық есебінің шешуіне
}

\begin{abstract}
Мақалада үзіліссіз функциялар класындағы жылуөткізгіштіктің бөлшекті-жүктемелі теңдеуі үшін шеттік есеп қарастырылған. Зерттеу әдістері шеттік есептерді интегралдық теңдеулерге келтіруге негізделген зерттеу болып табылады. Қойылған шеттік есеп дифференциалдық бөлікті айналдыру арқылы екінші текті Вольтерра интегралдық теңдеуіне келтірілген. Алынған теңдеудің ядросында арнайы функция бар. Сондай-ақ, жылуөткізгіштік теңдеуінің шеттік есебінің жүктелген қосылғышының бөлшек туындысы ретінің шектік жағдайлары зерттелді. Интегралдық теңдеудің шешуінің бар болуы мен жалғыздығы бастапқы шеттік есептің жүктелген қосылғышындағы бөлшек туындының ретіне байланысты екендігі көрсетілген.
\end{abstract}

Kiлm сөздер: жүктелген теңдеу, бөлшек туынды, жылуөткізгіштік теңдеуі, Вольтерра интегралдық теңдеуі, арнайы функция. 
М.Т. Космакова, С.А. Искаков, Л.Ж. Касымова

\section{К решению двумерной граничной задачи теплопроводности в вырождающейся области}

В статье рассмотрена краевая задача для дробно-нагруженного уравнения теплопроводности в классе непрерывных функций. Методы исследования базируются на подходе к исследованию краевых задач, основанном на их сведении к интегральным уравнениям. Поставленная краевая задача сведена к интегральному уравнению Вольтерра второго рода обращением дифференциальной части. Ядро полученного уравнения содержит специальную функцию. Также проведено исследование предельных случаев порядка дробной производной слагаемого с нагрузкой в уравнении теплопроводности краевой задачи. Показано, что существование и единственность решения интегрального уравнения зависят от порядка дробной производной в нагруженном слагаемом исходной краевой задачи.

Ключевые слова: нагруженное уравнение, дробная производная, уравнение теплопроводности, интегральное уравнение Вольтерра, специальная функция. 


\author{
V.P. Kvasnikov, S.V. Yehorov, T.Yu. Shkvarnytska \\ National Aviation University, Kyiv, Ukraine \\ (E-mail:kvp@nau.edu.ua, sehorov@gmail.com,tetyanashkvarnytska@gmail.com)
}

\title{
Technology for restoring functional dependencies to determine reliability parameters
}

\begin{abstract}
The problem of determining the properties of the object by analyzing the numerical and qualitative characteristics of a discrete sample is considered. A method has been developed to determine the probability of trouble-free operation of electronic systems for the case if the interpolation fields are different between several interpolation nodes. A method has been developed to determine the probability of trouble-free operation if the interpolation polynomial is the same for the entire interpolation domain. It is shown that local interpolation methods give more accurate results, in contrast to global interpolation methods. It is shown that in the case of global interpolation it is possible to determine the value of the function outside the given values by extrapolation methods, which makes it possible to predict the probability of failure. It is shown that the use of approximation methods to determine the probability of trouble-free operation reduces the error of the second kind. A method for analyzing the qualitative characteristics of functional dependences has been developed, which allows us to choose the optimal interpolation polynomial. With sufficient statistics, using the criteria of consent, it is possible to build mathematical models for the analysis of failure statistics of electronic equipment. Provided that the volume of statistics is not large, such statistics may not be sufficient and the application of consent criteria will lead to unsatisfactory results. Another approach is to use an approximation method that is applied to statistical material that was collected during testing or controlled operation. In this regard, it is extremely important to develop a method for determining the reliability of electronic systems in case of insufficiency of the collected statistics of failures of electronic equipment.
\end{abstract}

Keywords: approximation, interpolation, reliability of electronic equipment, statistical processing, restoration of functional dependence, estimation of reliability indicators, small sample, sufficiency of statistics.

\section{Introduction}

In order to increase the reliability and noise immunity of electronic systems operated in space and aviation, it is necessary to conduct numerical tests with high accuracy.

If it was possible to collect sufficient statistics, then using the criteria of agreement there is an opportunity to choose a mathematical apparatus with which you can analyze this series. But if the volume of statistics is not large, then such statistics may not be sufficient and the application of the consent criteria will lead to unsatisfactory results. The existing methods of studying the reliability of electronic equipment do not fully meet the requirements of practice and the level of production technology. It is explained by the fact that the values of real reliability indicators differ significantly from the forecast estimates, as the sufficiency of the collected statistics of failures is not taken into account.

Insufficient statistics make it impossible to apply the criteria of consent for the choice of the law of distribution of work to failure due to the large error of the second kind, with which the selected laws of distribution will align the collected statistics. Therefore, it is necessary to develop a method of data processing to study and solve this problem.

All of the above-mentioned leads to the need to solve the problem of restoring functional dependencies. Therefore, the problem of restoring functional dependences on a discrete experimentally obtained sample by local and global interpolation methods is relevant. Electronic systems are known to be highly reliable, and failure statistics are very difficult to collect. In the well-known publications of X. Changhui, R. Xiaoping, S. Xianfeng, G. Jingxiang on the study of reliability in [1] the modeling of the studied object by Kalman filtering method is

\footnotetext{
${ }^{*}$ Corresponding author.

E-mail: sehorov@gmail.com
} 
offered. But in the case of modeling the behavior of electronic systems, this method can lead to large errors in the simulation results. This is due to the fact that the technology of manufacturing electronic systems is very complex. In the electronic system there are a lot of complex processes, which are very difficult or impossible to take into account. For example, the software environment for modeling the operation of electronic circuits NI Multisim, in the case of building complex electronic circuits may not correctly model the processes occurring in electronic equipment. According to its properties, the Kalman filter will return more accurate results only if the data are normal in the distribution. But research data will not necessarily have a normal distribution. Therefore, the method described in [1] for modeling the behavior of electronic systems should be used only in the case of justification of the use of normal distribution.

Z. Zhengcheng, B. Narayanaswamy in [2] suggest determining of the probability of failure-free operation of the system using signatures, which are a function, the arguments of which are the operating time for failure. But the suggested method does not answer the question of the sufficiency of the collected statistics. H. Zhipeng, G. Jianbin, Z. Shengkui in [3] used a combination of Bayesian methods with system state vectors to determine the reliability of the system to reduce the number of calculations and the amount of statistics. But to determine the probability of failure, it cannot be recommended because the study [3] does not contain the calculation of the error that occurs if selected for data processing of a mathematical apparatus. F. Hailin, D. Jieyu in [4] suggests determining of the probability of failure-free operation of the system using signatures, as well as in [2], but the method suggested in [4] is suitable for solving problems of diagnosing and identifying problematic places during system operation.

S. Xujun, L. Xuezhi in [5] proposed on the basis of modeling of discrete events algorithm of modeling of reliability of system of a step-by-step mission with several states. The program algorithm includes the Markov process. But the degradation process that takes place in the system will not necessarily be Markov. Therefore, the reliability model for different types of systems will be individual.

Groezinger, A. Zimmermann in their research [6] tested the reliability of LEDs in the laboratory. A correlation analysis was used to process the test results. The presence of a correlation indicates the relationship between the data being analyzed, but does not determine the mathematical apparatus by which the experimental data can be processed. Of particular interest is the publication of X. Zhao, S. Wang, L. Sun [7] which suggested a method of planning tests for the reliability of finished products using Markov circles. However, in the production of complex electronic equipment, where it is necessary to control the parameters of the product at each stage of the technological cycle of production, this method is not suitable.

D. Zhou, H. Wang, F. Blaabjerg in [8] used the Weibull distribution to evaluate the reliability of mains electric filters and voltage converters and did not sufficiently substantiate their choice. For this reason, it is not known with what error the Weibull distribution will align the experimental data. In this case, it is necessary to check the plausibility of hypotheses and in case of insufficient statistics to solve the problem of restoring functional dependencies.

Kostanovsky V.V., Machalin I.A., Kozachuk O.D., Terentyeva I.A. in [9] have built a generalized probabilistic physical model of reliability of a two-level active phased array. Exponential and diffusion nonmonotonic distribution were used in [9] as models of failures of electronic components and transmission channels. However, [10] showed that the exponential and diffusion nonmonotonic distribution has no advantages along with other distributions with similar characteristics. Therefore, the use of exponential and diffusion nonmonotonic distribution in the study [9] is not justified.

Analysis of the literature [1-10] shows that the problem of restoring functional dependences on a discrete experimentally obtained sample by local and global interpolation methods is relevant.

Problem setting. It is necessary to develop a method of restoration of functional dependences in the conditions of insufficiency of statistical data by means of technologies of approximation and interpolation.

In order to achieve this goal, the following tasks were set:

- to investigate the existing methods of approximation;

- on the basis of research to develop a method for determining the probability of failure-free operation in case of insufficiency of statistics on a discrete experimentally obtained sample.

\section{Material and method}

At the first stage of data processing it is necessary to determine the sufficiency of statistics. This should be done according to the method described in [10]. If the statistics are insufficient, there are two options for solving this problem: 1 . Continue to collect failure statistics, in the case of actual observations, or simulate more failures. 2. Restoration of functional dependences by approximation methods. Suppose the values of some function at 
given points are known. You need to find intermediate values of this function, according to the approximation method. This is a task to restore the function. In addition, when performing calculations, it is convenient to replace complex functions with algebraic polynomials or other elementary functions, which are quite simple to calculate (problems on the approximation of the function) [11].

The formulation of the interpolation problem is as follows. In the interval $[a, b]$ the points $x_{i}, i=0,1, \ldots, N$ are given; $a \leq x_{i} \leq b$ and the value of the unknown function at these points $f_{i}, i=0,1, \ldots, N$. We need to find function $F(x)$ that takes the same values of $f_{i}$ at $x_{i}$. In this case, we look for $F(x)$ only on the segment $[a, b]$. If it is necessary to find a function outside the segment, then this is an extrapolation problem.

The problem has many solutions, because through the given points $\left(x_{i}, f_{i}\right), i=0,1, \ldots, N$, it is possible to draw infinitely many curves, each of which will be a graph of the function for which all interpolation conditions are fulfilled. The case of approximation of a function by polynomials is important for practice, [11]

$$
F(x)=a_{0}+a_{1} x+a_{2} x^{2}+\ldots+a_{m} x^{m} .
$$

All interpolation methods can be divided into local and global. In the case of local interpolation, a separate polynomial is constructed on each interval $\left[x_{i}{ }^{\lrcorner} 1, x_{i}\right]$. In the case of global interpolation, a single polynomial is found in the entire interval $[a, b]$.

On each segment $\left[x_{i \sim 1}, x_{i}\right]$ the interpolation polynomial is equal to a constant, namely the left or right value of the function.

For the left piecewise linear interpolation it is: $f(x)=f_{i-1}$, if i.e. $x_{i-1} \leq x<x_{i}[11,12]$

$$
F(x)=\left\{\begin{array}{l}
f_{0}, x_{0} \leq x<x_{1} \\
f_{1}, x_{1} \leq x<x_{2} \\
\cdots \\
f_{N-1}, x_{N-1} \leq x<x_{N} .
\end{array}\right.
$$

For the right piecewise linear interpolation it is: $F(x)=f_{i}$ if $x_{i-1}<x \leq x_{i}$, i.e. [11, 12].

$$
F(x)=\left\{\begin{array}{l}
f_{0}, x_{0}<x \leq x_{1} \\
f_{1}, x_{1}<x \leq x_{2} \\
\cdots \\
f_{N-1}, x_{N-1}<x \leq x_{N} .
\end{array}\right.
$$

Thus, the interpolation conditions are fulfilled. The constructed function is discontinuous, which limits its application.

The principle of piecewise-linear $[11,12]$ interpolation is as follows. At each interval $\left[x_{i-1}, x_{i}\right]$, the function is linear $F_{i}(x)=k_{i} x+l_{i}$. The values of the coefficients are from the fulfillment of the interpolation conditions at the ends of the segment: $f_{i}\left(x_{i-1}\right)=f_{i-1}, f_{i}\left(x_{i}\right)=f_{i}$. We get a system of equations: $k_{i} x_{i}+l_{i}=f_{i-1}$, where we find: $k_{i}=\frac{f_{i}-f_{i-1}}{x_{i}-x_{i-1}}, l_{i}=f_{i}-k_{i} x_{i}$ from. Therefore, the function $F(z)$ can be written as: $F(x)=\frac{f_{i}-f_{i-1}}{x_{i}-x_{i-1}} x+f_{i}-k_{i} x_{i}$ if $x_{i-1} \leq<x_{i}$ that is:

$$
F(x)=\left\{\begin{array}{l}
\frac{f_{1}-f_{0}}{x_{1}-x_{0}} x+f_{0}-k_{0} x_{0}, x_{0} \leq x_{i}<x_{1} \\
\frac{f_{2}-f_{1}}{x_{2}-x_{1}} x+f_{1}-k_{1} x_{1}, x_{1} \leq x_{1}<x_{2} \\
\cdots \\
\frac{f_{N}-f_{N-1}}{x_{N}-x_{N-1}} x+f_{N-1}-k_{N-1} x_{N-1}, x_{N-1} \leq x_{N-1}<x_{N} .
\end{array}\right.
$$

When using linear interpolation, you must first determine the interval in which the value of $\mathrm{x}$ falls, and then substitute it into the formula. The final function will be continuous, but the original derivative will be absent in each interpolation node. The error of such interpolation will be less than in the case of piecewise constant interpolation.

When interpolating functions, the condition of equality of values of interpolation polynomial and this function in interpolation nodes was used. If the original data are obtained as a result of experimental measurements, 
the requirement of exact coincidence, as a rule, does not apply because the data are not obtained accurately. In these cases, only an approximate fulfillment of the interpolation conditions can be required $\left.\mid F\left(x_{i}\right)-f\left(x_{i}\right)\right) \mid<\varepsilon$. This condition means that the interpolating function $\mathrm{F}(\mathrm{x})$ does not pass exactly through given points, but in some of their margins.

In this case, use a selection of empirical functions. The construction of an empirical function consists of two stages: the selection of the form of this function $\phi\left(x, a_{0}, a_{1}, \ldots, a_{m}\right)$, which contains unknown parameters $a_{0}, a_{1}, \ldots, a_{m}$, and the determination of the best in some sense arguments of this function. The type of function is sometimes known for physical reasons (for an elastic medium, the relationship between stress and strain). Or the type of function is chosen for geometric reasons: the experimental points are plotted on a graph and the general form of the dependence is roughly guessed by comparing the obtained curve with the graphs of known functions. Success here is largely determined by the experience and intuition of the researcher.

The case of approximation of a function by polynomials is important for practice [11-15], i.e.

$$
F(x)=a_{0}+a_{1} x+a_{2} x^{2}+\ldots+a_{m} x^{m} .
$$

Once the type of empirical dependence is selected, the degree of proximity to the empirical data is determined using a minimum of the sum of the squares of the deviations of the calculated and experimental data.

Suppose that for the initial datax $x_{i}, f_{i}, i=1, \ldots, N$ (numbering is better to start with one), the type of empirical dependence is chosen: $\phi\left(x, a_{0}, a_{1}, \ldots, a_{m}\right)$ with unknown coefficients $a_{0}, a_{1}, \ldots, a_{m}$. We write the sum of the squares of the deviations between the calculated by empirical formula and given experimental data:

$$
S\left(a_{0}, a_{1}, \ldots, a_{m}\right)=\sum_{i=1}^{N}\left(\phi\left(x, a_{0}, a_{1}, \ldots, a_{m}\right)-f_{i}\right)^{2} .
$$

We will find arguments $a_{0}, a_{1}, \ldots, a_{m}$ from the condition of a minimum of function $S\left(a_{0}, a_{1}, \ldots, a_{m}\right)$. This is the least squares method (LSM).

It is known that at the point of minimum all partial derivatives from $S$ to $a_{0}, a_{1}, \ldots, a_{m}$ are equal to zero $[11,15]$ :

$$
\frac{d S}{d a_{0}}=0, \frac{d S}{d a_{1}}=0, \ldots, \frac{d S}{d a_{m}}=0
$$

Consider the application of the LSM for a particular case, widely used in practice. As an empirical function, consider a polynomial $[11,15]$

$$
\phi(x)=a_{0}+a_{1} x+a_{2} x^{2}+\ldots+a_{m} x_{i}^{m},
$$

Formula (1) for determining the sum of squares of deviations will take the form [11, 15]:

$$
S\left(a_{0}, a_{1}, \ldots, a_{m}\right)=\sum_{i=1}^{N}\left(a_{0}+a_{1} x+a_{2} x^{2}+\ldots+a_{m} x^{m}-f_{i}\right)^{2}
$$

Calculate the derivatives $[11,15]$ :

$$
\left\{\begin{array}{l}
\frac{d S}{d a_{0}}=2 \sum_{i=1}^{N}\left(a_{0}+a_{1} x+a_{2} x^{2}+\ldots+a_{m} x^{m}-f_{i}\right) \\
\frac{d S}{d a_{1}}=2 \sum_{i=1}^{N}\left(a_{0}+a_{1} x+a_{2} x^{2}+\ldots+a_{m} x^{m}-f_{i}\right) x_{i} \\
\cdots \\
\frac{d S}{d a_{m}}=2 \sum_{i=1}^{N}\left(a_{0}+a_{1} x+a_{2} x^{2}+\ldots+a_{m} x^{m}-f_{i}\right) x_{i}^{m} .
\end{array}\right.
$$

Equating these expressions to zero and collecting the coefficients for the unknowns, we obtain a system of linear equations $[11,15]$ : 


$$
\left\{\begin{array}{l}
N a_{0}+a_{1} \sum_{i=1}^{N} x_{i}+a_{2} \sum_{i=1}^{N} x_{i}^{2}+\ldots+a_{m} \sum_{i=1}^{N} x_{i}^{m}=\sum_{i=1}^{N} f_{i} \\
a_{0} \sum_{i=1}^{N} x_{i}+a_{1} \sum_{i=1}^{N} x_{i}^{2}+a_{2} \sum_{i=1}^{N} x_{i}^{3}+\ldots+a_{m} \sum_{i=1}^{N} x_{i}^{m+1}=\sum_{i=1}^{N} x_{i} f_{i} \\
\ldots \\
a_{0} \sum_{i=1}^{N} x_{i}^{m}+a_{1} \sum_{i=1}^{N} x_{i}^{m+1}+a_{2} \sum_{i=1}^{N} x_{i}^{m+2}+\ldots+a_{m} \sum_{i=1}^{N} x_{i}^{2 m}=\sum_{i=1}^{N} x_{i}^{m} f_{i} .
\end{array}\right.
$$

This system of equations is called normal and is used to find the coefficients $a_{0}, a_{1}, . . a_{m}$.

As a rule, several empirical dependencies are chosen. The LSM finds the coefficients of these dependences and among them find the best for the minimum amount of deviations.

\section{Results of the research of approximation methods}

Selection of empirical functions. The specified values of the failure function of the electronic system (Table 1).

Table. 1 shows that $N=6$. You need to find empirical dependences: linear $\rho 1=a_{0}+a_{1} x$, quadratic $\rho 2=a_{0}+a_{1} x+a_{2} x^{2}$, hyperbolic $\rho 3=a_{0} \frac{a_{1}}{x}$ by the LSM and choose among them the best for the smallest sum of squares of deviations.

The system of normal equations for linear dependence:

$$
\left\{\begin{array}{l}
N a_{0}+a_{1} \sum_{i=1}^{N} x_{i}=\sum_{i=1}^{N} f_{i} \\
a_{0} \sum_{i=1}^{N} x_{i}+a_{1} \sum_{i=1}^{N} x_{i}^{2}=\sum_{i=1}^{N} x_{i} f_{i}
\end{array}\right.
$$

T a b l e 1

The number of failures and the corresponding probabilities

\begin{tabular}{|c|c|c|c|c|c|c|}
\hline Parameters used in the calculations & $x_{0}$ & $x_{1}$ & $x_{2}$ & $x_{3}$ & $x_{4}$ & $x_{5}$ \\
\hline$x$ (number of failures) & 1 & 2 & 3 & 4 & 8 & 10 \\
\hline$f$ (statistical probability of failure-free operation) & 0,1 & 0,15 & 0,2 & 0,3 & 0,45 & 0,7 \\
\hline
\end{tabular}

Given that $N=6$, and solving a system of linear equations, we obtain $a_{0}=-0,25 ; a 1=175$. Given that $\mathrm{N}=6$, and solving a system of linear equations, we obtain . Thus, the linear dependence has the form $\rho 1=-0,25+175 x$ (Fig. 1).

Calculate the sum of the squares of the deviations: $s_{1}=\sum_{i=0}^{6}\left(a_{0}+a_{1} x-f_{i}\right)^{2}=2,63$. Consider the quadratic dependence. The system of normal equations has the form:

$$
\left\{\begin{array}{l}
6 a_{0}+a_{1} \sum_{i=1}^{6} x_{i}+a_{2} \sum_{i=1}^{6} x_{i}^{2}=\sum_{i=1}^{6} f_{i} \\
a_{0} \sum_{i=1}^{6} x_{i}+a_{1} \sum_{i=1}^{6} x_{i}^{2}+a_{2} \sum_{i=1}^{6} x_{i}^{3}=\sum_{i=1}^{6} x_{i} f_{i} \\
a_{0} \sum_{i=1}^{6} x_{i}^{2}+a_{1} \sum_{i=1}^{6} x_{i}^{3}+a_{2} \sum_{i=1}^{6} x_{i}^{4}=\sum_{i=1}^{6} x_{i}^{2} f_{i}
\end{array}\right.
$$

Solving simultaneous linear algebraic equations we obtain $a_{0}=-0,101 ; a_{1}=14,982 ; a_{2}=0,162$. Thus, the quadratic dependence has the form: $\rho 2=-0,101+14,982 x+0,162 x^{2}$. Calculate the sum of the squares of the deviations: $s_{2}=\sum_{i=0}^{6}\left(a_{0}+a_{1} x+a_{2} x_{i}^{2}-f_{i}\right)^{2}=2,234$. 


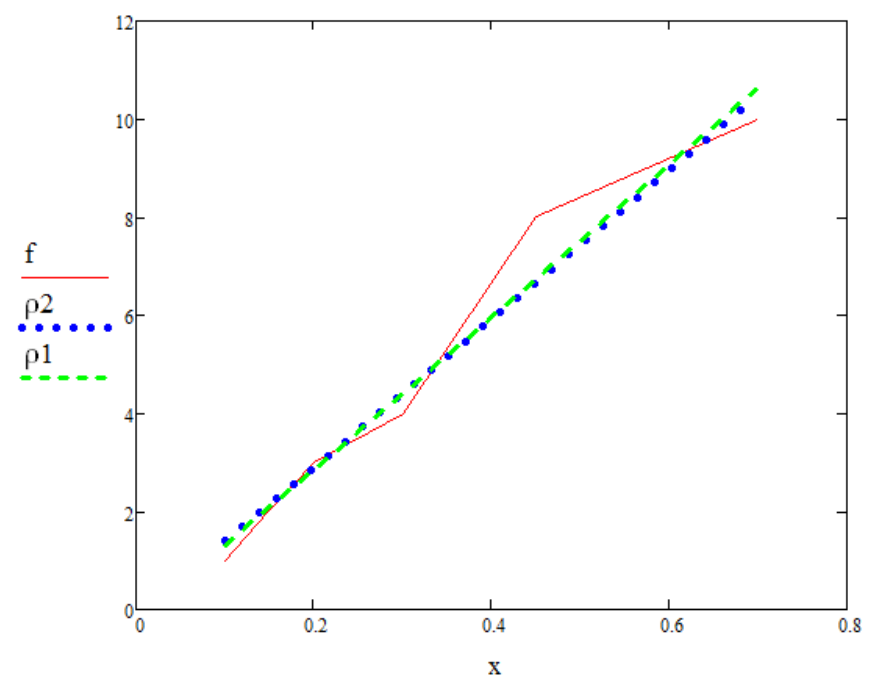

Figure 1. Approximation of experimental data by quadratic dependence: $f$ is the experimental data, $\rho 1$ - approximation by linear dependence, $\rho 2$ - approximation by quadratic dependence.

Let's make a system of normal equations for hyperbolic dependence. According to the LSM we find the sum of squares of deviations: $S_{3}=\sum_{i=0}^{N}\left(a_{0}+\frac{a_{1}}{x_{i}}-f_{i}\right)^{2}$. We make a system of normal equations:

$$
\left\{\begin{array}{l}
\frac{d S}{d a_{0}}=2 s_{3}=\sum_{i=1}^{6}\left(a_{0}+\frac{a_{1}}{x_{x_{i}}}-f_{i}\right)=0 \\
\frac{d S}{d a_{0}}=2 s_{3}=\sum_{i=1}^{6}\left(a_{0}+\frac{a_{1}}{x_{x_{i}}}-f_{i}\right) \frac{1}{x_{i}}=0 .
\end{array}\right.
$$

Solving the equations gives

$$
a_{0}=1,942 ; a 1=0,571 .
$$

The sum of deviations squares $S_{3}=-46,687$. Thus, the results of the calculations by the LSM show that it is best to approximate the curve by the quadratic dependence (Fig. 1).

As can be seen from Fig. 1 approximating curves $\rho 1$ and $\rho 2$ in appearance, in this case, it is very difficult to distinguish and very difficult to see which curve is better to approximate the experimental data. Therefore, it is very important to calculate the sum of the squares of the deviations.

\section{Conclusions}

As can be seen from Fig. 1 approximating curves describe a statistical series consisting of experimental data with some error. This error is characterized by the least squares check. In Fig. 1 it is difficult to see. In order to obtain more accurate results, it is necessary to use approximating curves of higher order, or local interpolation methods. The peculiarity of this method is that in the conditions of insufficiency of the collected statistics the probability of making an error of the second kind decreases due to the possibility to choose an approximating curve that can align the statistical series with the required accuracy. Analyzing the results of the research, it can be stated that the suggested method allows to effectively calculate the parameters of reliability in case of insufficiency of the collected statistics of failures.

The advantages of the suggested method are the following ones:

- it is possible to calculate the value of a function for any argument in short time with the required accuracy.

The disadvantages are:

- insufficient clarity in terms of the behavior of functional dependence;

- the analytical formula is often unknown;

- in some cases, the known functional dependence is too tedious for easy use in practice. 
But it should be noted that the disadvantages of the suggested method can be offset by the use of modern software and sufficiently powerful computer systems.

Due to the advent of computing systems that can handle multiple threads simultaneously, the prospect of developing approximation methods is the development of new methods for approximating numerical information with a multithreaded structure to solve problems in mathematical physics, such as grids, finite elements.

\section{References}

1 Changhui X. Generalized reliability measures of Kalman filtering for precise point positioning / X. Changhui, R. Xiaoping, S. Xianfeng, G.Jingxiang // Journal of Systems Engineering and Electronics. - 2013. - Vol. 24. - Issue 4. - P. 699-705. doi: 10.1109/JSEE.2013.00081

2 Zhengcheng Z. Representations for reliability functions of conditional coherent systems with INID components and ordered properties / Z.Zhengcheng, B. Narayanaswamy // Journal of Systems Engineering and Electronics. - 2015. - Vol. 26. - Issue 6. - P. 1316-1324. doi: 10.1109/JSEE.2015.

00144

3 Zhipeng H. Fully Bayesian reliability assessment of multi-state systems with overlapping data / H. Zhipeng, G. Jianbin, Z. Shengkui // Journal of Systems Engineering and Electronics. - 2017. - Vol. 28. Issue 1. - P. 187-198. doi: 10.21629/JSEE.2017.01.21

4 Hailin F. Reliability analysis for WSN based on a modular k-out-of-n system / F. Hailin, D. Jieyu // Journal of Systems Engineering and Electronics. - 2017. - Vol. 28. - Issue 2. - P. 407-412. doi: 10.21629/JSEE.2017.02.21

5 Xujun S. Reliability simulation and analysis of phased-mission system with multiple states / S. Xujun, L. Xuezhi // Journal of Systems Engineering and Electronics. - 2019. - Vol. 30. - Issue 3. - P. 624-632. doi: 10.21629/JSEE.2019.03.19

6 Soltani M. Reliability Study and Thermal Performance of LEDs on Molded Interconnect Devices (MID) and PCB / M. Soltani, M. Freyburger, R.Kulkarni, R. Mohr, T. Groezinger, A. Zimmermann // IEEE Access. - 2018. - Vol. 6. - P. 51669-51679. doi: ACCESS.2018.2869017

7 Zhao X. Single and Sequential Sampling Plans for Multi-Attribute Products and Multi-Class Lot in Reliability Test [Text] / X. Zhao, S. Wang, L. Sun // IEEE Access. - 2019. - Vol. 7. - P. 81145-81155. doi: 10.1109/ACCESS.2019.2923316

8 Zhou D. Mission Profile Based System-Level Reliability Analysis of DC/DC Converters for a Backup Power Application [Text] / D. Zhou, H. Wang, F.Blaabjerg // IEEE Transactions on Power Electronics. - 2018. - Vol. 33. - Issue 9. - P. 8030-8039. doi: TPEL.2017.2769161

9 Костановский В.В. Построение обобщенной вероятностно-физической модели надежности двухуровневой активной фазированной антенной решетки / В.В. Костановский, И.А. Мачалин, О.Д. Козачук, И.А. Терентьева // Восточно-Европейский журн. передовых технологий. - 2019. - Т. 3. - № 9 (99). - C. 31-40. doi: 10.15587/1729-4061.2019.168525

10 Єгоров С.В. Дослідження метода визначення безвідмовності технічних систем з використанням моделювання випробувань / С.В. Єгоров, Т.Ю. Шкварницька // Технологічний аудит та резерви виробництва. - 2016. - № 2/2(28). - С. 25-29. doi: 10.15587/2312-8372.2016.66481

11 Калиткин Н.Н. Численные методы / Н.Н. Калиткин. - СПб.: БХВ-Петербург, 2011. - 592 с.

12 Гусак А.А. Справочник по высшей математике / А.А. Гусак, Г.М. Гусак, Е.А. Бричикова. - Мн.: ТетраСистемс, 1999. - 640 с.

13 Анисимов-Спиридонов Д.Д. Методы и модели больших систем оптимального планирования и управления / Д.Д. Анисимов-Спиридонов. - М.: Наука, 1969. - 960 с.

14 Юдин Д.Б. Линейное программирование / Д.Б. Юдин, Е.Г. Гольштейн. - М.: Наука, 1969. - 424 с.

15 Венецкий И.Г. Основные математико-статистические понятия и формулы в экономическом анализе: справоч. / И.Г. Венецкий, В.И. Венецкая. - М.: Статистика, 1979. - 447 с. 


\title{
Сенімділік параметрлерін анықтау үшін функционалдық тәуелділікті қалпына келтіру технологиясы
}

\begin{abstract}
Дискретті іріктеу бойынша сандық және сапалық сипаттамаларды талдау арқылы объектінің қасиеттерін анықтау мәселесі қарастырылды. Интерполяцияның бірнеше түйіндері арасындағы интерполяциялық көпмүшелер әр түрлі болған жағдайда электронды жүйелердің ақаусыз жұмыс істеу ықтималдығын анықтау әдісі жасалды. Интерполяция полиномы интерполяцияның бүкіл ауданы үшін бірдей болған жағдайда, ақаусыз жұмыс ықтималдығын анықтау әдісі жасалды. Жергілікті интерполяция әдістері жаһандық интерполяция әдістеріне қарағанда дәлірек нәтиже беретіні көрсетілген. Ғаламдық интерполяция жағдайында экстраполяция әдістерімен берілген мәндерден тыс функцияның мәнін анықтауға болатындығы көрсетілген, бұл ақаусыз жұмыс ықтималдығын болжау мүмкіндігін береді. Ақаусыз жұмыс ықтималдығын анықтау үшін аппроксимациялау әдістерін қолдану екінші типтегі қателіктің төмендеуіне әкелетіндігі көрсетілген. Функционалды тәуелділіктердің сапалық сипаттамаларын талдау әдісі жасалды, бұл оңтайлы интерполяциялық көпмүшені таңдауға мүмкіндік береді. Жеткілікті статистикамен келісім критерийлерін қолдана отырып, электронды жабдықтың істен шығу статистикасын талдаудың математикалық модельдерін құруға болады. Егер статистика көлемі үлкен болмаса, онда мұндай статистика жеткіліксіз болуы мүмкін және келісім критерийлерін қолдану қанағаттанарлықсыз нәтижелерге әкеледі. Тағы бір тәсіл - тестілеу кезінде жиналған статистикалық материалға немесе бақыланатын пайдаланудағы материалға қолданылатын аппроксимациялау әдісін қолдану. Осыған байланысты электронды жабдықтың істен шығу статистикасы жеткіліксіз болған жағдайда электронды жүйелердің сенімділігін анықтау әдісін жасау өте маңызды.
\end{abstract}

Kiлm сөздер: электронды жабдықтың жақындауы, интерполяциясы, сенімділігі, статистикалық өңдеу, функционалды тәуелділікті қалпына келтіру, сенімділік көрсеткіштерін бағалау, шағын іріктеме, статистиканың жеткіліктілігі.

\author{
В.П. Квасников, С.В. Егоров, Т.Ю. Шкварницкая
}

\section{Технология восстановления функциональных зависимостей для определения параметров надёжности}

\begin{abstract}
Рассмотрена задача определения свойств объекта путём анализа числовых и качественных характеристик по дискретной выборке. Разработан метод определения вероятности безотказной работы электронных систем для случая, когда интерполяционные полиномы между несколькими узлами интерполяции различны. Разработан метод определения вероятности безотказной работы в случае, когда интерполяционный полином для всей области интерполяции одинаковый. Показано, что методы локальной интерполяции дают более точные результаты в отличие от методов глобальной интерполяции. Показано, что в случае глобальной интерполяции есть возможность определить значение функции за пределами заданных значений методами экстраполяции, что дает возможность прогнозирования вероятности безотказной работы. Показано, что использование методов аппроксимации для определения вероятности безотказной работы приводит к уменьшению ошибки второго рода. Разработан метод анализа качественных характеристик функциональных зависимостей, что позволяет выбирать оптимальный интерполяционный полином. При достаточной статистике, с использованием критериев согласия, можно строить математические модели анализа статистики отказов электронной аппаратуры. При условии, что объем статистики небольшой, такая статистика может оказаться недостаточной, и применение критериев согласия приведёт к неудовлетворительным результатам. Другой подход состоит в использовании метода аппроксимации, который применяется к статистическому материалу, который был собран во время испытаний, или подконтрольной эксплуатации. В связи с этим крайне важно разработать метод определения надежности электронных систем в случае недостаточности собранной статистики отказов электронной аппаратуры.
\end{abstract}

Ключевые слова: аппроксимация, интерполяция, надёжность электронной аппаратуры, статистическая обработка, восстановление функциональной зависимости, оценка показателей надёжности, малая выборка, достаточность статистики. 


\section{References}

1 Changhui, X., Xiaoping, R., Xianfeng, S., \& Jingxiang, G. (2013). Generalized reliability measures of Kalman filtering for precise point positioning. Journal of Systems Engineering and Electronics, 24(4), 699-705. DOI: 10.1109/JSEE.2013.00081

2 Zhengcheng, Z., \& Narayanaswamy, B. (2015). Representations for reliability functions of conditional coherent systems with INID components and ordered properties. Journal of Systems Engineering and Electronics, 26(6), 1316-1324. DOI: 10.1109/JSEE. 2015.00144

3 Zhipeng, H., Jianbin, G., \& Shengkui, Z. (2017). Fully Bayesian reliability assessment of multi-state systems with overlapping data. Journal of Systems Engineering and Electronics, 28(1), 187-198. DOI: 10.21629/JSEE.2017.01.21

4 Hailin, F., \& Jieyu, D. (2017). Reliability analysis for WSN based on a modular k-out-of-n system. Journal of Systems Engineering and Electronics, 28(2), 407-412. DOI: 10.21629/JSEE.2017.02.21

5 Xujun, S., \& Xuezh,i L. (2019). Reliability simulation and analysis of phased-mission system with multiple states. Journal of Systems Engineering and Electronics, 30(3), 624-632. DOI: 10.21629/JSEE.2019.03.19

6 Soltani, M., Freyburger, M., Kulkarni, R., Mohr, R., Groezinger, T., \& Zimmermann, A. (2018). Reliability Study and Thermal Performance of LEDs on Molded Interconnect Devices (MID) and PCB. IEEE Access, 6, 51669-51679. DOI: 10.1109/ACCESS.2018.2869017

7 Zhao, X., Wang, S., \& Sun, L. (2019). Single and Sequential Sampling Plans for Multi-Attribute Products and Multi-Class Lot in Reliability Test. IEEE Access, 7, 81145-81155.

DOI: 10.1109 /ACCESS.2019.2923316

8 Zhou, D., Wang, H., \& Blaabjerg, F. (2018). Mission Profile Based System-Level Reliability Analysis of DC/DC Converters for a Backup Power Application. IEEE Transactions on Power Electronics, 33(9), 8030-8039. DOI: 10.1109/TPEL.2017.2769161

9 Kostanovsky, V., Machalin, I., Kozachuk, O., \& Terentyeva, I. (2019). Construction of a generalized probabilistic-physical model of reliability of a two-level active phased antenna array. Eastern-European Journal of Enterprise Technologies, 3, 9(99), 31-40. DOI: 10.15587/1729-4061.2019.168525

10 Yehorov, S.V., \& Shkvarnytska, T.Yu. (2016). Research of method for determining reliability of technical system using simulation tests. Technology audit and production reserves, 2/2(28), 25-29. DOI: 10.15587 /2312-8372.2016.66481

11 Kalitkin, N.N. (2011). Chislennye metody [Numerical methods]. Saint Petersburg: BHV-Peterburg [in Russian].

12 Gusak, A.A., Gusak, G.M., \& Brichikova, E.A. (1999). Spravochnik po vysshei matematike [Handbook of Higher Mathematics]. Minsk: TetraSistems [in Russian].

13 Anisimov-Spiridonov, D. D. (1969). Metody i modeli bolshikh sistem optimalnoho planirovaniia $i$ upravleniia [Methods and models of large systems of optimal planning and control]. Moscow: Nauka [in Russian].

14 Yudin, D. B., \& Golshtejn, E. G. (1969). Lineinoe prohrammirovanie [Linear programming]. Moscow: Nauka [in Russian].

15 Veneckij, I.G., \& Veneckaya, V.I. (1979). Osnovnye matematiko-statisticheskie poniatiia $i$ formuly $v$ ekonomicheskom analize: spravochnik [Basic mathematical and statistical concepts and formulas in economic analysis: Handbook]. Moscow: Statistika [in Russian]. 


\author{
M.B. Muratbekov ${ }^{1, *}$, A.O. Suleimbekova ${ }^{2}$ \\ ${ }^{1}$ Taraz Regional University named after M.Kh. Dulaty, Taraz, Kazakhstan; \\ ${ }^{2}$ L.N. Gumilyev Eurasian National University, Nur-Sultan, Kazakhstan \\ (E-mail: musahan_m@mail.ru, suleimbekovaa@mail.ru)
}

\title{
On the existence of the resolvent and separability of a class of the Korteweg-de Vriese type linear singular operators
}

\begin{abstract}
Partial differential equations of the third order are the basis of mathematical models of many phenomena and processes, such as the phenomenon of energy transfer of hydrolysis of adenosine triphosphate molecules along protein molecules in the form of solitary waves, i.e. solitons, the process of transferring soil moisture in the aeration zone, taking into account its movement against the moisture potential. In particular, this class includes the nonlinear Korteweg-de Vries equation, which is the main equation of modern mathematical physics. It is known that various problems have been studied for the Korteweg-de Vries equation and many fundamental results obtained. In this paper, issues about the existence of a resolvent and separability (maximum smoothness of solutions) of a class of linear singular operators of the Korteweg-de Vries type in the case of an unbounded domain with strongly increasing coefficients are investigated.
\end{abstract}

Keywords: resolvent, Korteweg-de Vries type singular operator, separability.

\section{Introduction}

The solvability of boundary value problems for odd order differential equations, in particular, to the Korteweg-de Vries equation is dealt with in a significant literature [1-9] and the papers cited there.

In contrast to these interesting papers, this article deals with the problem of the existence of a resolvent and the separability of a class of linear singular operators of the Korteweg-de Vries type in the case of an unbounded domain with strongly increasing coefficients.

Note that if the boundary regime operates sufficiently long, then due to the friction inherent in any real physical system, the influence of the initial data weakens with time. Thus, we arrive at a problem without initial conditions [10].

Therefore, taking this remark into account in the present work, we consider the differential operator

$$
L u+\lambda u=\frac{\partial u}{\partial y}+R_{2}(y) \frac{\partial^{3} u}{\partial x^{3}}+R_{1}(y) \frac{\partial u}{\partial x}+R_{0}(y) u+\lambda u
$$

initially defined on $C_{0, \pi}^{\infty}(\bar{\Omega})$, where $\bar{\Omega}=\{(x, y):-\pi \leq x \leq \pi,-\infty<y<\infty\}, \lambda \geq 0$.

$C_{0, \pi}^{\infty}$ is a set of infinitely differentiable functions satisfying the conditions:

$$
u_{x}^{(i)}(-\pi, y)=u_{x}^{(i)}(\pi, y), \quad i=0,1,2
$$

and compactly supported with respect to the variable $y$.

Further assume that the coefficients $R_{0}(y), R_{1}(y), R_{2}(y)$ satisfy the following conditions:

i) $\left.R_{0}(y) \geq \delta_{0}\right)>0, R_{1}(y) \geq \delta_{1}>0,-R_{2}(y) \geq \delta_{2}>0$ are continuous functions in $R(-\infty,+\infty)$;

ii) $\mu_{0}=\sup _{|y-t| \leq 1} \frac{R_{0}(y)}{R_{0}(t)}<\infty, \mu_{1}=\sup _{|y-t| \leq 1} \frac{R_{1}(y)}{R_{1}(t)}<\infty, \mu_{2}=\sup _{|y-t| \leq 1} \frac{R_{2}(y)}{R_{2}(t)}<\infty$.

The operator $L+\lambda I$ admits closure in $L_{2}(\Omega)$, which we also denote by $L+\lambda I$.

Theorem 1. Let the condition i) be fulfilled. Then the operator $L+\lambda I$ is continuously invertible in $L_{2}(\Omega)$ for $\lambda \geq 0$.

\footnotetext{
${ }^{*}$ Corresponding author.

E-mail: musahan_m@mail.ru
} 
Definition. We called the operator $L$ is separable in $u \in D(L)$ if the estimate

$$
\left\|\frac{\partial u}{\partial y}\right\|_{L_{2}(\Omega)}+\left\|R_{2}(y) \frac{\partial^{3} u}{\partial x^{3}}\right\|_{L_{2}(\Omega)}+\left\|R_{1}(y) \frac{\partial u}{\partial x}\right\|_{L_{2}(\Omega)}+\left\|R_{0}(y) u\right\|_{L_{2}(\Omega)} \leq C\left(\|L u\|_{2}+\|u\|_{L_{2}(\Omega)}\right),
$$

holds for $u(x, y)$, where $\mathrm{C}$ is independent of $u(x, y),\|\cdot\|_{2}$ is the norm of $L_{2}(\Omega)$.

Theorem 2. Let conditions i) - ii) be fulfilled. Then the operator $L$ is separable.

Example. Let $R_{0}(y)=|y|+1, R_{1}(y)=e^{|y|}, R_{2}(y)=-10 \cdot e^{|y|},-\infty<y<\infty$. It is easy to verify that all the conditions of Theorem 2 are satisfied. Consequently, the operator $L$ is separable, i.e.

$$
\left\|\frac{\partial u}{\partial y}\right\|_{L_{2}(\Omega)}+\left\|10 \cdot e^{|y|} \frac{\partial^{3} u}{\partial x^{3}}\right\|_{L_{2}(\Omega)}+\left\|e^{|y|} \frac{\partial u}{\partial x}\right\|_{L_{2}(\Omega)}+\|(|y|+1) u\|_{L_{2}(\Omega)} \leq C\left(\|L u\|_{L_{2}(\Omega)}+\|u\|_{L_{2}(\Omega)}\right),
$$

$C$ is a constant.

\section{Auxiliary lemmas and inequalities}

Lemma 2.1. Let the condition i) be fulfilled and $\lambda \geq 0$. Then the inequality

$$
\|(L+\lambda I) u\|_{L_{2}(\Omega)} \geq\left(\delta_{0}+\lambda\right)\|u\|_{L_{2}(\Omega)},
$$

holds for all $u \in D(L)$, where $\delta_{0}>0$.

Proof. Let $u \in C_{0, \pi}^{\infty}(\bar{\Omega})$ and consider the scalar product

$$
\begin{gathered}
<(L+\lambda I) u, u>=\int_{\Omega}\left(\frac{\partial u}{\partial y}+R_{2}(y) \frac{\partial^{3} u}{\partial x^{3}}+R_{1}(y) \frac{\partial u}{\partial x}+\left(R_{0}(y)+\lambda\right) u\right) u d x d y= \\
=\int_{\Omega} \frac{\partial u}{\partial y} u d x d y+\int_{\Omega} R_{2}(y) \frac{\partial^{3} u}{\partial x^{3}} u d x d y+\int_{\Omega} R_{1}(y) \frac{\partial u}{\partial x} u d x d y+\int_{\Omega}\left(R_{0}(y)+\lambda\right) u^{2} d x d y .
\end{gathered}
$$

Since $u \in C_{0, p i}^{\infty}(\Omega)$ and (2) holds, then the following equalities:

$$
\int_{\Omega} \frac{\partial u}{\partial y} u d x d y=0 ; \quad \int_{\Omega} R_{1}(y) \frac{\partial u}{\partial x} u d x d y=0 ; \quad \int_{\Omega} R_{2}(y) \frac{\partial^{3} u}{\partial x^{3}} u d x d y=0 .
$$

hold. Using the equality (5) from (4) we have

$$
<(L+\lambda I) u, u>=\int_{\Omega}\left(R_{0}(y)+\lambda\right) u^{2} d x d y .
$$

From (6), using the Cauchy-Bunyakovsky inequality and the condition i), we obtain

$$
\|(L+\lambda I) u\|_{L_{2}(\Omega)} \geq\left(\delta_{0}+\lambda\right)\|u\|_{L_{2}(\Omega)},
$$

for all $u \in C_{0, \pi}^{\infty}(\bar{\Omega})$. By virtue of the continuity of the norm, the last estimate holds for all $u \in D(L)$. Lemma 2.1 is proved.

Remark. Due to the realness of the coefficients of (1) the estimate (7) holds for complex-valued functions. Consider the operator

$$
\left(l_{n, j}+\lambda I\right) z=z^{\prime}(y)+\left(-i n^{3} R_{2, j}(y)+i n R_{1, j}(y)+R_{0, j}(y)\right) z(y),
$$

where $R_{2, j}(y), R_{1, j}(y), R_{0, j}(y)$ are bounded periodic functions of the same period $\Delta_{j}=(j-1, j+1), j=0$, $\pm 1, \pm 2, z(y) \in C_{0}^{\infty}(R), z(y)=u(y)+i \vartheta(y)$.

Lemma 2.2. Let the condition i) be fulfilled. Then the operator $\left(l_{n, j}+\lambda I\right)$ considered on $C_{0}^{\infty}(R)$ is a closable operator in $L_{2}(R)$.

Proof. Let $u_{n} \in C_{0}^{\infty}(R)$ and $u_{n} \stackrel{L_{2}(R)}{\rightarrow} 0,\left(l_{n, j}+\lambda I\right) u_{n} \stackrel{L_{2}(R)}{\rightarrow} \vartheta$. If you use the operator $\left(l_{n, j}+\lambda I\right)^{*}$ formally associated with the operator $\left(l_{n, j}+\lambda I\right)$, then for arbitrary function $\omega \in C_{0}^{\infty}(R)$ 


$$
<\left(l_{n, j}+\lambda I\right) u_{n}, \omega>=<u_{n},\left(l_{n, j}+\lambda I\right)^{*} \omega>
$$

where $\left(l_{n, j}+\lambda I\right)^{*} \omega=-\omega^{\prime}+\left(i n^{3} R_{2, j}(y)-i n R_{1, j}(y)+R_{0, j}(y)\right) \vartheta=0$.

Hence

$$
<\vartheta, \omega>=<0,\left(l_{n, j}+\lambda I\right)^{*} \omega>=0
$$

Consequently, $\langle\vartheta, \omega\rangle=0$ for any $\omega \in C_{0}^{\infty}(R)$ and it means $\vartheta=0$. Lemma 2.2 is proved.

Lemma 2.3. Let the condition i) be fulfilled. Then the estimate

$$
\left\|\left(l_{n, j}+\lambda I\right) z\right\|_{2} \geq\left(\delta_{0}+\lambda\right)\|z\|_{2}
$$

holds for all $z(y) \in D\left(l_{n, j}+\lambda I\right),\|\cdot\|_{2}$ is the norm of $L_{2}(R)$.

Proof. Let $z(y) \in C_{0}^{\infty}(R)$ and $z(y)=u(y)+i \vartheta(y)$. Consider the scalar product

$$
\begin{gathered}
<\left(l_{n, j}+\lambda I\right) z, z>=\int_{-\infty}^{\infty}\left(z^{\prime}+\left(-i n^{3} R_{2, j}(y)+i n R_{1, j}(y)+R_{0, j}(y)+\lambda\right) z\right) \bar{z} d y= \\
=\int_{-\infty}^{\infty} z^{\prime}(y) z(y) d y+\int_{-\infty}^{\infty}\left(-i n^{3} R_{2, j}(y)+i n R_{1, j}(y)+R_{0, j}(y)+\lambda\right)|z|^{2} d y= \\
=i\left(\int_{-\infty}^{\infty} u \vartheta^{\prime}(y) d y+\int_{-\infty}^{\infty}\left(-i n^{3} R_{2, j}(y)+i n R_{1, j}(y)\right)|z|^{2} d y\right)+\int_{-\infty}^{\infty}\left(R_{0, j}(y)+\lambda\right)|z|^{2} d y
\end{gathered}
$$

Hence, using the properties of complex numbers, we have

$$
\left|<\left(l_{n, j}+\lambda I\right) z, z>\right| \geq\left.\left|\int_{-\infty}^{\infty}\left(R_{0, j}(y)+\lambda\right)\right| z\right|^{2} d y \mid .
$$

Since $R_{0, j}(y)$ does not change the sign, then

$$
\left|<\left(l_{n, j}+\lambda I\right) u, u>\right| \geq \int_{-\infty}^{\infty}\left|\left(R_{0, j}(y)+\lambda\right)\right||z|^{2} d y .
$$

By virtue of condition i) and using the Cauchy-Bunyakovsky inequality from the latter inequality, we have

$$
\left\|\left(l_{n, j}+\lambda I\right) z\right\|_{2} \geq\left(\delta_{0}+\lambda\right)\|z\|_{2} .
$$

Lemma 2.3 is proved.

Lemma 2.4. Let the condition i) be fulfilled.Then the operator $\left(l_{n, j}+\lambda I\right)$ has a continuous inverse operator $\left(l_{n, j}+\lambda I\right)^{-1}$ defined on the whole $L_{2}(R)$.

Proof. By the estimate (8) it suffices to show that the range is dense in $L_{2}(R)$. Let's assume that the range is not dense in $L_{2}(R)$. Then there is an element $\vartheta \in L_{2}(R)$ such that $\left\langle\left(l_{n, j}+\lambda I\right) u, \vartheta\right\rangle=0$ for all $u \in D\left(l_{n, j}\right)$. This means that

$$
\left(l_{n, j}+\lambda I\right)^{*} \vartheta=-\vartheta^{\prime}+\left(i n^{3} R_{2, j}(y)-i n R_{1, j}(y)+R_{0, j}(y)\right) \vartheta=0 .
$$

in terms of distribution theory. By the periodicity of functions $R_{0}(y), R_{1}(y), R_{2}(y)$, we have that $\left(i n^{3} R_{2, j}(y)-\right.$ $\left.-i n R_{1, j}(y)+R_{0, j}(y)\right) \vartheta \in L_{2}(R)$. From this and from (10) it follows that $\vartheta \in W_{2}^{1}(R)$, where $W_{2}^{1}(R)$ is the Sobolev space. The general theory of the embedding theorems implies that

$$
\lim _{|y| \rightarrow \infty} \vartheta(y)=0 .
$$

Now, using the equality (11) and the arguments used in the proof of the estimate (9), we obtain that

$$
\left\|\left(l_{n, j}+\lambda I\right)^{*} \vartheta\right\|_{2} \geq \delta_{0}\|\vartheta\|_{2} .
$$

From estimates (12) and (10) it follows that $\vartheta=0$. Lemma 2.4 is proved.

Let $\left\{\varphi_{j}\right\}_{j=-\infty}^{\infty} \in C_{0}^{\infty}(R)$ is a set of such functions that $\varphi_{j}(y) \geq 0, \sup p \varphi_{j} \subseteq \Delta_{j}(j \in Z), \sum_{j=-\infty}^{\infty} \varphi_{j}^{2}(y)=1$.

Here we note immediately that any point $y \in R$ can belong to no more than three segments from the system of segments $\left\{\operatorname{supp} \varphi_{j}\right\}[11,12]$. 
Assume that

$$
\begin{gathered}
K_{\lambda} f=\sum_{j=-\infty}^{\infty} \varphi_{j}(y)\left(l_{n, j}+\lambda I\right)^{-1} \varphi_{j} f, \\
B_{\lambda} f=\sum_{j=-\infty}^{\infty} \varphi_{j}^{\prime}(y)\left(l_{n, j}+\lambda I\right)^{-1} \varphi_{j} f, \quad f \in C_{0}^{\infty}(R), \quad \lambda \geq 0 .
\end{gathered}
$$

It is easy to verify that

$$
\left(l_{n}+\lambda I\right) K_{\lambda} f=f+\sum_{j} \varphi_{j}^{\prime}(y)\left(l_{n, j}+\lambda I\right)^{-1} \varphi_{j} f
$$

where

$$
\left(l_{n}+\lambda I\right) z=-z^{\prime}(y)+\left(-i n^{3} R_{2}(y)+i n R_{1}(y)+R_{0}(y)\right) z, z \in D\left(l_{n}\right) .
$$

Lemma 2.5. Let the condition i) be fulfilled. Then there is a number $\lambda_{0}>0$ such that $\left\|B_{\lambda}\right\|_{2 \rightarrow 2}<1$ for all $\lambda \geq \lambda_{0}$

Proof. Let $f \in C_{0}^{\infty}(R)$. Only functions $\varphi_{j-1}, \varphi_{j}, \varphi_{j+1}$ are nonzero in the interval $\overline{\Delta_{j}}(j \in Z)$, consequently:

$$
\left\|B_{\lambda} f\right\|_{L_{2}(R)}^{2}=\int_{-\infty}^{\infty}\left|\sum_{j=-\infty}^{\infty} \varphi_{j}^{\prime}(y)\left(l_{n, j}+\lambda I\right)^{-1} \varphi_{j} f\right|^{2} d y \leq \sum_{j=-\infty}^{\infty} \int_{\Delta_{j}}\left|\sum_{k=j-1}^{j+1}\left[\varphi_{k}^{\prime}(y)\left(l_{n, k}+\lambda I\right)^{-1} \varphi_{k} f\right]\right|^{2} d y .
$$

Hence, using the obvious inequality $(a+b+c)^{2} \leq 3\left(a^{2}+b^{2}+c^{2}\right)$ and estimate (8), we have:

$$
\begin{gathered}
\left\|B_{\lambda} f\right\|_{L_{2}(R)}^{2} \leq \sum_{j=-\infty}^{\infty} \int_{\Delta_{j}}\left|\sum_{k=j-1}^{j+1}\left[\varphi_{k}^{\prime}\left(l_{n, k}+\lambda I\right)^{-1} \varphi_{k} f\right]\right|^{2} d y \leq 9 \sum_{j=-\infty}^{\infty}\left\|\varphi_{j}^{\prime}\left(l_{n, j}+\lambda I\right)^{-1} \varphi_{j} f\right\|_{L_{2}\left(\Delta_{j}\right)}^{2} \leq \\
\leq 9 \sum_{j=-\infty}^{\infty}\left\|\varphi_{j}^{\prime}\left(l_{n, j}+\lambda I\right)^{-1} \varphi_{j} f\right\|_{L_{2}(R)}^{2} \leq 9 \cdot c \sum_{j=-\infty}^{\infty}\left\|\left(l_{n, j}+\lambda I\right)^{-1}\right\|_{L_{2}(R) \rightarrow L_{2}(R)}^{2} \cdot\left\|\varphi_{j} f\right\|_{L_{2}(R)}^{2} \leq \\
\leq \frac{9 \cdot c}{\left(\delta_{0}+\lambda\right)^{2}} \cdot \int_{-\infty}^{\infty}\left(\sum_{j} \varphi_{j}^{2}\right)|f|^{2} d y=\frac{9 \cdot c}{\left(\delta_{0}+\lambda\right)^{2}} \cdot\|f\|_{L_{2}(R)}^{2} .
\end{gathered}
$$

This implies that

$$
\left\|B_{\lambda}\right\|_{L_{2}(R) \rightarrow L_{2}(R)} \leq \frac{9 \cdot c}{\left(\delta_{0}+\lambda\right)^{2}} .
$$

From (14) it follows that it is easy to find a number $\lambda_{0}>0$, such that $\lambda \geq \lambda_{0},\left\|B_{\lambda}\right\|_{L_{2}(R) \rightarrow L_{2}(R)}<1$. Lemma 2.5 is proved.

Now consider the operator

$$
\left(l_{n}+\lambda I\right) z=z^{\prime}(y)+\left(-i n^{3} R_{2}(y)+i n R_{1}(y)+R_{0}(y)\right) z(y),
$$

where $z(y)=u(y)+i \vartheta(y), z(y) \in C_{0}^{\infty}(R),(R=(-\infty, \infty))$.

Lemma 2.6. Let the condition i) be fulfilled. Then the estimate

$$
\left\|\left(l_{n}+\lambda I\right) z\right\|_{2} \geq\left(\delta_{0}+\lambda\right)\|z\|_{2} .
$$

holds for all $z \in D\left(l_{n}\right)$. 2.6 .

Proof. We obtain the proof of Lemma 2.3 by reproducing the computations and argument used in Lemma

Lemma 2.7. Let the condition i) be fulfilled. Then the operator $l_{n}+\lambda I$ for $\lambda \geq \lambda_{0}$ is boundedly invertible and the equality

$$
\left(l_{n}+\lambda I\right)^{-1}=K_{\lambda}\left(I-B_{\lambda}\right)^{-1} .
$$

holds for the inverse operator $\left(l_{n}+\lambda I\right)^{-1}$.

The proof of Lemma 2.7 follows from the representations (13), (15) and Lemma 2.5 
3 On the existence of the resolvent. Proof of Theorem 1

Lemma 2.7 implies that

$$
u_{k}(x, y)=\sum_{n=-k}^{k}\left(l_{n}+\lambda I\right)^{-1} f_{n}(y) e^{i n x}
$$

is the solution to the problem

$$
\begin{gathered}
(L+\lambda I) u_{k}(x, y)=f_{k}(x, y), \\
u_{k}^{(i)}(-\pi, y)=u_{k}^{(i)}(\pi, y), i=0,1,2
\end{gathered}
$$

where $f_{k}(x, y) \stackrel{L_{2}(R)}{\rightarrow} f(x, y), f_{k}(x, y)=\sum_{n=-k}^{k} f_{n}(y) \cdot e^{i n x}, i^{2}=-1,\left(l_{n}+\lambda I\right)^{-1}$ is the inverse operator to the operator $l_{n}+\lambda I$. Using the inequality (3), we obtain

$$
\left\|u_{k}(x, y)\right\|_{2} \leq \frac{1}{\left(\delta_{0}+\lambda\right)}\left\|f_{k}(x, y)\right\|_{2} .
$$

Since $f_{k}(x, y) \stackrel{L_{2}(R)}{\rightarrow} f(x, y)$, then from (18) we find that

$$
\left\|u_{k}-u_{m}\right\|_{2} \leq \frac{1}{\delta_{0}}\left\|f_{k}-f_{m}\right\|_{2} \rightarrow 0, \quad k, m \rightarrow \infty .
$$

Hence, by the completeness of $L_{2}(\Omega)$, it follows that there exist a unique function $u \in L_{2}(\Omega)$ such that

$$
u_{k}(x, y) \rightarrow u(x, y) \text { as } k \rightarrow \infty .
$$

(17) and (19) imply that

$$
u(x, y)=(L+\lambda I)^{-1} f(x, y)=\sum_{n=-\infty}^{\infty}\left(l_{n}+\lambda I\right)^{-1} f_{n}(y) e^{i n x}
$$

is a strong solution for:

$$
\begin{gathered}
(L+\lambda I) u=f, \\
u^{(i)}(-\pi, y)=u^{(i)}(\pi, y), \quad i=0,1,2 .
\end{gathered}
$$

for any $f \in L_{2}(\Omega)$.

Let us recall the definition of a strong solution. The function $u \in L_{2}(\Omega)$ is called a strong solution of the problem (21)-(22), if there exists a $\left\{u_{k}\right\}_{k=1}^{\infty} \subset C_{0, \pi}^{\infty}(\Omega)$ such that

$$
\left\|u_{k}-u\right\|_{L_{2}(\Omega)} \rightarrow 0,\left\|(L+\lambda I) u_{k}-f\right\|_{L_{2}(\Omega)} \rightarrow 0 \text { as } k \rightarrow \infty .
$$

Now, it is not difficult to verify that the formula $(20)$ is the inverse operator to the closed operator $L+\lambda I$.

Lemma 2.8. [13]. Let the operator $L+\lambda_{0} I\left(\lambda_{0}>0\right)$ is boundedly invertible in $L_{2}(\Omega)$ and the estimate $\|(L+\lambda I) u\|_{L_{2}(\Omega)} \geq\|u\|_{L_{2}(\Omega)}, u \in D(L+\lambda I)$ holds for $\lambda \in\left[0, \lambda_{0}\right]$. Then the operator $L: L_{2}(\Omega) \rightarrow L_{2}(\Omega)$ is also boundedly invertible.

This and Lemma 2.1 implies that Theorem 1 holds for all $\lambda \geq 0$. Theorem 1 is completely proved.

\section{On the separability of the operator}

First, we give the lemmas that reduce the question of separability of an operator with unbounded coefficients to the case of an operator with periodic coefficients.

Lemma 2.9. Let $z(y) \in D\left(l_{n}+\lambda I\right)$ and $z(y)=u(y)+i \vartheta(y)$, then $i n^{3} R_{2}(y) z(y) \in L_{2}(R)$ if and only if $n^{3} R_{2}(y) u(y) \in L_{2}(R)$ and $n^{3} R_{2}(y) \vartheta(y) \in L_{2}(R)$.

Proof. Necessity. Let $i n^{3} R_{2}(y) z(y) \in L_{2}(R)$. Then

$$
\begin{gathered}
\left\|i n^{3} R_{2}(y) z(y)\right\|_{2}^{2}=\int_{-\infty}^{\infty}\left|i n^{3} R_{2}(y) z(y)\right|^{2} d y=\int_{-\infty}^{\infty}\left|n^{6} R_{2}^{2}(y) \| z(y)\right|^{2} d y= \\
=\int_{-\infty}^{\infty}\left|n^{6} R_{2}^{2}(y)\right|\left(|u|^{2}+|\vartheta|^{2}\right) d y=\left\|n^{3} R_{2}(y) u\right\|_{2}^{2}+\left\|n^{3} R_{2}(y) \vartheta\right\|_{2}^{2}
\end{gathered}
$$


It follows that $n^{3} R_{2}(y) u(y) \in L_{2}(R)$ and $n^{3} R_{2}(y) \vartheta(y) \in L_{2}(R)$.

Sufficiency. Let $n^{3} R_{2}(y) u(y) \in L_{2}(R)$ and $n^{3} R_{2}(y) \vartheta(y) \in L_{2}(R)$. Then

$$
\left\|i n^{3} R_{2}(y) z(y)\right\|_{2}^{2}=\left\|n^{3} R_{2}(y) u\right\|_{2}^{2}+\left\|n^{3} R_{2}(y) \vartheta\right\|_{2}^{2}
$$

Hence $i n^{3} R_{2}(y) z(y) \in L_{2}(R)$. Lemma is proved.

Remark. This Lemma is also true for $i n^{3} R_{1}(y) z(y)$.

By virtue of this Lemma, we consider the operator

$$
\left(l_{n, j}+\lambda I\right) u=u^{\prime}(y)+\left(-i n^{3} R_{2, j}(y)+i n R_{1, j}(y)+R_{0}(y)+\lambda\right) u(y),
$$

in the space $C_{0}^{\infty}(R)$ the set of infinitely differentiable, finite, and real-valued functions, where $R_{0, j}(y), R_{1, j}(y)$, $R_{2, j}(y)$ are bounded periodic coefficients of the same period $\Delta_{j}=(j-1, j+1), j= \pm 0, \pm 1, \pm 2 \ldots$

Lemma 2.10. Let the condition i) be fulfilled. Then the estimates:

$$
\left\|\left(l_{n, j}+\lambda I\right) u(y)\right\|_{2} \geq R_{0}\left(y_{j}\right)\|u\|_{2}, \quad n=0, \pm 1, \pm 2 \ldots
$$

where $R_{0}\left(y_{j}\right)=\min _{y \in \overline{\Delta_{j}}} R_{0, j}(y)$;

$$
\left\|\left(l_{n, j}+\lambda I\right) u(y)\right\|_{2} \geq|n| R_{1}\left(\overline{y_{j}}\right)\|u\|_{2}, \quad n=0, \pm 1, \pm 2 \ldots
$$

where $R_{1}\left(\overline{y_{j}}\right)=\min _{y \in \overline{\Delta_{j}}} R_{1, j}(y)$;

$$
\left\|\left(l_{n, j}+\lambda I\right) u(y)\right\|_{2} \geq|n|^{3} R_{2}\left(\overline{\overline{y_{j}}}\right)\|u\|_{2} \quad n=0, \pm 1, \pm 2 \ldots
$$

where $R_{2}\left(\overline{\overline{y_{j}}}\right)=\min _{y \in \overline{\Delta_{j}}}\left|R_{2, j}(y)\right|,\|\cdot\|_{2}$ is the norm of $L_{2}(R)$.

Proof. Let $u(y) \in C_{0}^{\infty}(R)$. Given that $\int_{-\infty}^{\infty} u^{\prime}(y) u(u) d y=0$ and reproducing the computations used in the proof of Lemma 2.1, we have:

$$
\left|<\left(l_{n, j}+\lambda I\right) u, u>\right|=\left|\int_{-\infty}^{\infty}\left(-i n^{3} R_{2, j}(y)+i n R_{1, j}(y)+R_{0, j}(y)+\lambda\right) u^{2} d y\right|
$$

From estimate (26) we find that

$$
\left|<\left(l_{n, j}+\lambda I\right) u, u>\right| \geq\left.\left|\int_{-\infty}^{\infty}\left(R_{0, j}(y)+\lambda\right)\right| u\right|^{2} d y \mid \geq \min _{y \in \overline{\Delta_{j}}} R_{0}(y)\|u\|_{2}^{2},
$$

Using the Cauchy-Bunyakovsky inequality, we obtain from (27) that

$$
\left.\| l_{n, j}+\lambda I\right) u\left\|_{2} \geq R_{0}\left(y_{j}\right)\right\| u \|_{2},
$$

where $R_{0}\left(y_{j}\right)=\min _{y \in \overline{\Delta_{j}}} R_{0}(y)$.

The proof of the inequality (28) follows from the inequality (22) of lemma 2.10.

Further, from the inequality (26) we find

$$
\left|<\left(l_{n, j}+\lambda I\right) u, u>\right| \geq\left|-i \int_{-\infty}^{\infty}\left(n^{3} R_{2, j}(y)+n R_{1, j}(y)\right) u^{2} d y\right| .
$$

Here we used the properties of complex numbers. From (29), by virtue of the condition i), we obtain the following inequalities:

$$
\begin{gathered}
\left|<\left(l_{n, j}+\lambda I\right) u, u>\right| \geq\left|\int_{-\infty}^{\infty} n R_{1, j}(y) u^{2} d y\right| \geq|n| R_{1}\left(\overline{y_{j}}\right)\|u\|_{2}^{2}, \\
\left|<\left(l_{n, j}+\lambda I\right) u, u>\right| \geq\left.\left.\left|\int_{-\infty}^{\infty}\left(-n^{3} R_{2, j}(y)\right)\right| u\right|^{2} d y|\geq| n\right|^{3}\left|R_{2}\left(\overline{\overline{y_{j}}}\right)\right|\|u\|_{2}^{2},
\end{gathered}
$$

where $R_{1}\left(\overline{y_{j}}\right)=\min _{y \in \overline{\Delta_{j}}} R_{1, j}(y), R_{2}\left(\overline{\overline{y_{j}}}\right)=\min _{y \in \overline{\Delta_{j}}}\left|R_{2, j}(y)\right|$. From these inequalities and using the Cauchy-Bunyakovsky inequality, we find that:

Lemma 2.10 is proved.

$$
\begin{gathered}
\left.\| l_{n, j}+\lambda I\right) u\left\|_{2} \geq|n| R_{1}\left(\overline{y_{j}}\right)\right\| u \|_{2} ; \\
\left.\| l_{n, j}+\lambda I\right) u\left\|_{2} \geq n^{3}\left|R_{2}\left(\overline{\overline{y_{j}}}\right)\right|\right\| u \|_{2} .
\end{gathered}
$$


Lemma 2.11. Let the condition i) be fulfilled and $\lambda \geq \lambda_{0}, \alpha=0,1,2,3, p(y)$ defined on $R$ continuous function. Then the estimate

$$
\left\|p(y)|n|^{\alpha}\left(l_{n}+\lambda I\right)^{-1}\right\|_{L_{2}(R) \rightarrow L_{2}(R)}^{2} \leq c(\lambda) \sup _{j \in Z}\left\|p(y)|n|^{\alpha} \varphi_{j}\left(l_{n, j}+\lambda I\right)^{-1}\right\|_{L_{2}\left(\Delta_{j}\right) \rightarrow L_{2}\left(\Delta_{j}\right)}^{2} .
$$

Proof. Let $f \in C_{0}^{\infty}(R)$. From the representation (16), taking the properties of the functions $\varphi_{j}(j \in Z)$ into account, we have:

$$
\begin{gathered}
\left\|p(y)|n|^{\alpha}\left(l_{n}+\lambda I\right)^{-1} f\right\|_{L_{2}(R)}^{2}=\left\|p(y)|n|^{\alpha} K_{\lambda}\left(I-B_{\lambda}\right)^{-1} f\right\|_{L_{2}(R)}^{2}= \\
=\left.\left.\int_{-\infty}^{\infty}|p(y)| n\right|^{\alpha} \sum_{\{j\}} \varphi_{j}\left(l_{n, j}+\lambda I\right)^{-1} \varphi_{j}\left(I-B_{\lambda}\right)^{-1} f\right|^{2} d y
\end{gathered}
$$

As it is known that on $\Delta_{j}(j \in Z)$ the interval only functions $\varphi_{j-1}, \varphi_{j}, \varphi_{j+1}$ are nonzero, therefore

$$
\begin{gathered}
\left\|p(y)|n|^{\alpha}\left(l_{n}+\lambda I\right)^{-1} f\right\|_{L_{2}(R)}^{2} \leq\left.\left.\sum_{j=-\infty}^{\infty} \int_{\Delta_{j}}|p(y)| n\right|^{\alpha} \sum_{j-1}^{j+1} \varphi_{j}\left(l_{n, j}+\lambda I\right)^{-1} \varphi_{j}\left(I-B_{\lambda}\right)^{-1} f\right|^{2} d y \leq \\
\leq 9 \sum_{j=-\infty}^{\infty}\left\|\left.|p(y)| n\right|^{\alpha} \varphi_{j}\left(l_{n, j}+\lambda I\right)^{-1} \varphi_{j}\left(I-B_{\lambda}\right)^{-1} f\right\|_{L_{2}\left(\Delta_{j}\right)}^{2} \leq \\
\leq 9 \sup _{j \in Z}\left\|p(y)|n|^{\alpha} \varphi_{j}\left(l_{n, j}+\lambda I\right)^{-1}\right\|_{L_{2}\left(\Delta_{j}\right)}^{2} \cdot \sum_{j=-\infty}^{\infty} \int_{\Delta_{j}}\left|\varphi_{j}\left(I-B_{\lambda}\right)^{-1} f\right|^{2} d y \leq \\
\leq 9 \sup _{j \in Z}\left\|p(y)|n|^{\alpha} \varphi_{j}\left(l_{n, j}+\lambda I\right)^{-1}\right\|_{L_{2}\left(\Delta_{j}\right)}^{2} \cdot \int_{-\infty}^{\infty}\left(\sum_{j} \varphi_{j}^{2}\right)\left|\left(I-B_{\lambda}\right)^{-1} f\right|^{2} d y
\end{gathered}
$$

Since $\left(\sum_{j} \varphi_{j}^{2}\right)=1$, then from (31) we have

$$
\begin{gathered}
\left\|p(y)|n|^{\alpha}\left(l_{n}+\lambda I\right)^{-1} f\right\|_{L_{2}(R)}^{2} \leq 9 \sup _{j \in Z}\left\|p(y)|n|^{\alpha} \varphi_{j}\left(l_{n, j}+\lambda I\right)^{-1}\right\|_{L_{2}\left(\Delta_{j}\right)}^{2} \cdot \int_{-\infty}^{\infty}\left|\left(I-B_{\lambda}\right)^{-1} f\right|^{2} d y \leq \\
\leq 9 \sup _{j \in Z}\left\|p(y)|n|^{\alpha} \varphi_{j}\left(l_{n, j}+\lambda I\right)^{-1}\right\|_{L_{2}\left(\Delta_{j}\right)}^{2} \cdot\left\|\left(I-B_{\lambda}\right)^{-1}\right\|_{2 \rightarrow 2}^{2} \cdot\|f\|_{2}^{2} .
\end{gathered}
$$

Lemma 2.5 implies that $\left\|I-B_{\lambda}\right\|_{2 \rightarrow 2}^{2}<c(\lambda)$. From this and from (32) we obtain that

$$
\left.\| p(y)|n|^{\alpha}\right]\left(l_{n, j}+\lambda I\right)^{-1}\left\|_{L_{2}(R) \rightarrow L_{2}(R)} \leq 9 \cdot c(\lambda) \sup _{j \in Z}\right\| p(y)|n|^{\alpha} \varphi_{j}\left(l_{n, j}+\lambda I\right)^{-1} \|_{L_{2}\left(\Delta_{j}\right)}^{2} .
$$

Lemma 2.11 is proved.

Lemma 2.12. Let the conditions i)-ii) be fulfilled. Then the following estimates:

$$
\begin{gathered}
\left\|R_{0}(y)\left(l_{n}+\lambda I\right)_{-1}\right\|_{L_{2}(R) \rightarrow L_{2}(R)} \leq C_{0}<\infty ; \\
\left\|R_{1}(y)|n|\left(l_{n}+\lambda I\right)_{-1}\right\|_{L_{2}(R) \rightarrow L_{2}(R)} \leq C_{1}<\infty ; \\
\left\|R_{2}(y)|n|^{3}\left(l_{n}+\lambda I\right)_{-1}\right\|_{L_{2}(R) \rightarrow L_{2}(R)} \leq C_{2}<\infty ;
\end{gathered}
$$

hold, where $C_{0}, C_{1}, C_{1}$ are independent of $n(n=0, \pm 1, \pm 2 \ldots)$.

Proof. (30) shows that the operator $R_{0}\left(l_{n}+\lambda I\right)$ is bounded, if $\sup _{j \in Z}\left\|R_{0}(y) \varphi_{j}\left(l_{n, j}+\lambda I\right)^{-1}\right\|_{L_{2}\left(\Delta_{j}\right)}$ is bounded. Therefore, we will estimate the last expression

$$
\begin{gathered}
\left\|R_{0}(y) \varphi_{j}\left(l_{n}+\lambda I\right)^{-1}\right\|_{L_{2}(R) \rightarrow L_{2}(R)}^{2} \leq C(\lambda) \sup _{j \in Z}\left\|R_{0}(y) \varphi_{j}\left(l_{n, j}+\lambda I\right)^{-1}\right\|_{L_{2}\left(\Delta_{j}\right)}^{2} \leq \\
\leq C(\lambda) \sup _{j \in Z} \max _{y \in \Delta_{j}}\left|R_{0}(y) \varphi_{j}\right|^{2}\left\|\left(l_{n, j}+\lambda I\right)^{-1}\right\|_{L_{2}\left(\Delta_{j}\right)}^{2} \leq C(\lambda) \sup _{j \in Z} \max _{y \in \Delta_{j}} R_{0}^{2}(y)\left\|\left(l_{n, j}+\lambda I\right)^{-1}\right\|_{L_{2}\left(\Delta_{j}\right)}^{2} .
\end{gathered}
$$


Hence, taking the inequality (23) and the condition ii) into account, we find that

$$
\left\|R_{0}(y)\left(l_{n}+\lambda I\right)^{-1}\right\|_{L_{2}(R) \rightarrow L_{2}(R)}^{2} \leq C(\lambda) \sup _{|y-t| \leq 1} \frac{R_{0}^{2}(y)}{R_{0}^{2}(t)}<C(\lambda) \cdot \mu_{0}^{2} \leq C_{0}^{2}<\infty .
$$

The last estimate proves the inequality (33) of Lemma 2.12 .

Let us prove the inequality (34). Using Lemma 2.11, we have:

$$
\begin{gathered}
\left\|R_{1}(y)|n|\left(l_{n}+\lambda I\right)^{-1}\right\|_{L_{2}(R) \rightarrow L_{2}(R)}^{2} \leq C(\lambda) \sup _{j \in Z}\left\|R_{1}(y)|n| \varphi_{j}\left(l_{n, j}+\lambda I\right)^{-1}\right\|_{L_{2}\left(\Delta_{j}\right) \rightarrow L_{2}\left(\Delta_{j}\right)}^{2} \leq \\
\leq C(\lambda) \sup _{j \in Z} \max _{y \in \overline{\Delta_{j}}} R_{1}^{2}(y)|n|^{2}\left\|\left(l_{n, j}+\lambda I\right)^{-1}\right\|_{L_{2}\left(\Delta_{j}\right) \rightarrow L_{2}\left(\Delta_{j}\right)}^{2} .
\end{gathered}
$$

Hence, using (24), as well as considering condition ii), we find that

$$
\left\|R_{1}(y)|n|\left(l_{n}+\lambda I\right)^{-1}\right\|_{L_{2}(R) \rightarrow L_{2}(R)}^{2} \leq C(\lambda) \cdot \mu_{1}^{2} \leq C_{1}^{2}<\infty .
$$

The inequality (34) is proved.

Let us prove the inequality (35). Reproducing the computations arguments used in the proof of the inequalities (33), (34) and using the inequality (25), we obtain

$$
\begin{gathered}
\left\|R_{2}(y)|n|^{3}\left(l_{n}+\lambda I\right)^{-1}\right\|_{L_{2}(R) \rightarrow L_{2}(R)}^{2} \leq C(\lambda) \sup _{j \in Z}\left\|R_{2}(y)|n|^{3} \varphi_{j}\left(l_{n, j}+\lambda I\right)^{-1}\right\|_{L_{2}\left(\Delta_{j}\right) \rightarrow L_{2}\left(\Delta_{j}\right)}^{2} \leq \\
\leq C(\lambda) \cdot \mu_{2}^{2} \leq C_{2}^{2}<\infty .
\end{gathered}
$$

The inequality (35) is proved. Lemma 2.12 is completely proved.

\section{Proof of Theorem 2}

The representation (20) implies that

$$
R_{0}(y) u(x, y)=R_{0}(y)(L+\lambda I)^{-1} f(x, y)=\sum_{n=-\infty}^{\infty} R_{0}(y)\left(l_{n}+\lambda I\right)^{-1} f_{n}(y) \cdot e^{i n x} .
$$

Calculate the norm $R_{0}(y)(L+\lambda I)^{-1} f$ :

$$
\begin{gathered}
\left\|R_{0}(y)(L+\lambda I)^{-1} f\right\|_{L_{2}(\Omega)}^{2}=\left\|\sum_{n=-\infty}^{\infty} R_{0}(y)\left(l_{n}+\lambda I\right)^{-1} f_{n}(y) \cdot e^{i n x}\right\|_{L_{2}(\Omega)}^{2} \leq \\
\leq 2 \pi \sum_{n=-\infty}^{\infty}\left\|R_{0}(y)\left(l_{n}+\lambda I\right)^{-1} f_{n}(y) \cdot e^{i n x}\right\|_{L_{2}(\Omega)}^{2} .
\end{gathered}
$$

From this and from Lemma 2.12 we have that

$$
\left\|R_{0}(y)(L+\lambda I)^{-1} f\right\|_{L_{2}(\Omega)}^{2} \leq 2 \pi \sum_{n=-\infty}^{\infty}\left\|R_{0}(y)\left(l_{n}+\lambda I\right)^{-1}\right\|_{L_{2}(\Omega)}^{2} \cdot\left\|f_{n}(y)\right\|_{L_{2}(\Omega)}^{2} \leq C_{0}^{2}\|f\|_{L_{2}(\Omega)}^{2} .
$$

From the last estimate, it follows that

$$
\left\|R_{0}(y) u(x, y)\right\|_{L_{2}(\Omega)}=\left\|R_{0}(y)(L+\lambda I)^{-1} f(x, y)\right\|_{L_{2}(\Omega)} \leq C_{0}\left\|(L+\lambda I)^{-1} u\right\|_{L_{2}(\Omega)}
$$

where $(L+\lambda I) u=f$.

Similarly, by virtue of the estimate (34) we have:

$$
\left\|R_{1}(y) \frac{\partial u}{\partial x}\right\|_{L_{2}(\Omega)} \leq C_{1}\left\|(L+\lambda I)^{-1} u\right\|_{L_{2}(\Omega)} .
$$


Similarly, repeating the above calculations, we get that

$$
\left\|R_{1}(y) \frac{\partial^{3} u}{\partial x^{3}}\right\|_{L_{2}(\Omega)}^{2} \leq C_{2}\left\|(L+\lambda I)^{-1} u\right\|_{L_{2}(\Omega)} .
$$

Now the inequalities (36)-(38) imply that

$$
\begin{gathered}
\left\|\frac{\partial u}{\partial y}\right\|_{L_{2}(\Omega)}=\left\|(L+\lambda I)-R_{2}(y) \frac{\partial^{3} u}{\partial x^{3}}-R_{1}(y) \frac{\partial u}{\partial y}-R_{0}(y) u-\lambda u\right\|_{L_{2}(\Omega)} \leq\|(L+\lambda I)\|_{L_{2}(\Omega)}+ \\
+\left\|R_{2}(y) \frac{\partial^{3} u}{\partial x^{3}}\right\|_{L_{2}(\Omega)}+\left\|R_{1}(y) \frac{\partial u}{\partial y}\right\|_{L_{2}(\Omega)}+\left\|R_{0}(y) u\right\|_{L_{2}(\Omega)}+\|\lambda u\|_{L_{2}(\Omega)} \leq\|(L+\lambda I) u\|_{L_{2}(\Omega)}+ \\
+C_{2}\|(L+\lambda I) u\|_{L_{2}(\Omega)}+C_{1}\|(L+\lambda I) u\|_{L_{2}(\Omega)}+C_{0}\|(L+\lambda I) u\|_{L_{2}(\Omega)}+\lambda\|(L+\lambda I) u\|_{L_{2}(\Omega)} \leq \\
\leq C(\lambda)\|(L+\lambda I) u\|_{L_{2}(\Omega)} .
\end{gathered}
$$

We obtain from the inequalities (36)-(39) that

$$
\left\|\frac{\partial u}{\partial y}\right\|_{L_{2}(\Omega)}+\left\|R_{2}(y) \frac{\partial^{3} u}{\partial x^{3}}\right\|_{L_{2}(\Omega)}+\left\|R_{1}(y) \frac{\partial u}{\partial y}\right\|_{L_{2}(\Omega)}+\left\|R_{0}(y) u\right\|_{L_{2}(\Omega)} \leq C(\lambda)\left(\|L u\|_{L_{2}(\Omega)}+\|u\|_{L_{2}(\Omega)}\right) .
$$

Theorem 2 is completely proved.

\section{References}

1 Temam R. Sur un probleme non linearie / R. Temam // Math. Pures. Apple. - 1969. - 48. - № 2. P. 159-172.

2 Лионс Ж.Л. Некоторые методы решения нелинейных краевых задач / Ж.Л. Лионс. - М.: Мир, 1972. $-586 \mathrm{c}$.

3 Дубинский Ю.А. Об одной абстрактной теореме и ее приложения к краевым задачам для неклассических уравнений / Ю.А.Дубинский // Матем. сб. - 1969. - 79(121). - № 1. - С. 91-117.

4 Юрчук Н.И. О граничных задачах для уравнений, содержащихся в главной части оператор вида $d^{2 m+1} / d t^{2 m+1} /$ Н.И. Юрчук // Дифференциальные уравнения. - 1974. - 10. - № 4. - С. 759-762.

5 Пятков С.Г. Об одном линейном уравнении неклассического типа высокого порядка / С.Г. Пятков // Препринт им. СО АН СССР. - 1981. - 24 с.

6 Bona J.Y. A nonhomogeneous boundary-value problem for the Korteweg-de Vries equation posed on a finite domain / J.Y. Bona, Shu Ming Sun, Bing-Yu Zhang // Comm. Partial Differential Equations. 2003. - 28. - №7-8. - P. 1391-1436.

7 Бубнов В.А. Разрешимость в целом нелинейных граничных задач для уравнения Кортевега-де Фриза в ограниченной области / В.А. Бубнов // Дифференциальные уравнения. $-1980 .-16 .-$ № 1 . - C. $34-41$.

8 Похожаев С.И. О некоторых весовых тождествах для решений обобщенных уравнений Кортевега-де Фриза / С.И.Похожаев // Математические заметки. - 2011. - 89. - Вып. 3. - С. 393-409.

9 Муратбеков М.Б. О существовании и аппроксимативных свойствах решений полупериодической задачи Дирихле для одного класса нелинейных вырождающихся уравнений неклассического типа / М.Б. Муратбеков, Г.К. Рахимова, А.Б. Шыракбаев // Матем. журн. - 2011. - 13. - № 2 (48). C. $95-109$.

10 Тихонов А.Н. Уравнения математической физики / А.Н. Тихонов, А.А. Самарский. - М.: Наука, 1972. $-735 \mathrm{c}$.

11 Отелбаев М. Коэрцитивные оценки и теоремы разделимости для эллиптических уравнений в $R^{n} /$ М. Отелбаев // Тр. Матем. ин-та АН СССР. - 1983. - 161. - С. 195-217.

12 Muratbekov M. On the existence of a resolvent and separability for a class of singular hyperbolic type differential operators on an unbounded domain / M. Muratbekov, M. Otelbaev // Eurasian mathematical journal. - 2016. - 7. - No I. - P. 50-67. 
13 Ахиезер Н.И. Теория линейных операторов в гильбертовом пространстве / Н.И. Ахиезер, И.М. Глазман. - М.: Наука, 1966. - 644 с.

М.Б. Муратбеков, А.О. Сулеймбекова

\title{
Кортевег-де Фриз типті сызықты сингулярлы операторлардың бір класының бар болуы және бөліктенуі туралы
}

\author{
Мақалада шексіз облыста коэффициенттері шексіздікте жылдам өсетін Кортвег-де Фриза типті сыз- \\ ықты оператор зерттелген. Жоғарыдағы көрсетілген дифференциалдық оператор үшін төмендегі \\ сұрақтар қарастырылған: \\ - резольвентаның бар болуы; \\ - оператордың бөліктенуі, яғни оператордың анықталу облысындағы функциялардың тегістігі зерт- \\ телген.
}

Kiлm сөздер: резольвента, Коревег-де Фриз типті сингулярлы оператор, бөліктену.

М.Б. Муратбеков, А.О. Сулеймбекова

\section{О существовании резольвенты и разделимости одного класса линейных сингулярных операторов типа Кортевега-де Фриза}

\author{
В статье исследован линейный дифференциальный оператор типа Кортевега-де Фриза в случае не- \\ ограниченной области с сильно растущими коэффициентами на бесконечности. Для указанного выше \\ оператора изучены следующие вопросы: \\ - существование резольвенты; \\ - разделимость оператора, т.е. гладкость функций из области определения изучаемого оператора. \\ Ключевые слова: резольвента, сингулярный оператор типа Кортевега-де Фриза, разделимость.
}

\section{References}

1 Temam, R. (1969). Sur un probleme non linearie. J. Math.Pures. Apple, 48(2), 159-172.

2 Lyons, J.L. (1972). Nekotorye metody resheniia nelineinykh kraevykh zadach /Some methods for solving nonlinear boundary value problems]. Moscow: Mir [in Russian].

3 Dubinsky, Yu.A. (1969). Ob odnoi abstraktnoi teorii i ee prilozheniiakh k kraievym zadacham dlia neklassicheskikh uravnenii [On an abstract theorem and its applications to boundary value problems for nonclassical equations]. Matematicheskii sbornik - Mathematical collection, 79(121), 1, 91-117 [in Russian].

4 Yurchuk, N.I. (1974). O hranichnykh zadachakh dlia uravnenii, soderzhashchikh v hlavnoi chasti operator vida $d^{2 m+1} / d t^{2 m+1}$ [On boundary value problems for equations containing operators of the form $d^{2 m+1}$ $/ d t^{2 m+1}$ in the main part]. Differentsialnye uravneniia - Differential equation., 10(4), $759-762$ [in Russian].

5 Pyatkov, S.G. (1981). Ob odnom lineinom uravnenii neklassicheskoho tipa vysokoho poriadka [On a non-classical type high-order linear equation]. Preprint imeni SO AN SSSR - Preprint of Institute of Mathematics, Siberian Branch of the USSR Academy of Sciences, 24 [in Russian].

6 Bona, J.Y., Shu Ming Sun, \& Bing-Yu Zhang (2003). A nonhomogeneous boundary-value problem for the Korteweg-de Vries equation posed on a finite domain. Comm. Partial Differential Equations., 28(7-8), 1391-1436. 
7 Bubnov, B.A. (1979). Razreshimost v tselom nelineinykh hranichnykh zadach dlia uravneniia Kortevehade Friza v ohranichennoi oblasti [Solvability in the large of nonlinear boundary value problems for the Korteweg-de Vries equation in a bounded domain]. Differentsialnye uravneniia - Differential equation, 16(1), 34-41 [in Russian].

8 Pokhozhaev, S.I. (2011). O nekotorykh vesovykh tozhdestvakh dlia reshenii obobshchennykh uravnenii Korteveha-de Friza [Weighted identities for solutions of generalized Korteweg-de Vries equations]. Matematicheskiie zametki - Math. Notes, 89(3), 393-409 [in Russian].

9 Muratbekov, M.B., Rakhimova, G.K., \& Shyrakbaev, A.B. (2011). O sushchestvovanii i approksimativnykh svoistvakh reshenii poluperiodicheskoi zadachi Dirikhle dlia odnoho klassa nelineinykh vyrozhdaiushchikhsia uravnenii neklassicheskoho tipa [On the existence and approximation properties of solutions of the semi-periodic Dirichlet problem for a class of nonlinear degenerate non-classical equations]. Matematicheskii zhurnal - Math journal, 13, 2(48), 95-109 [in Russian].

10 Tikhonov, A.N., \& Samarskii, A.A. (1972). Uravneniia matematicheskoi fiziki [Equations of mathematical physics]. Moscow: Nauka [in Russian].

11 Otelbaev, M. (1983). Koertsitivnye otsenki i teoremy razdelimosti dlia ellipticheskikh uravnenii v $R^{n}$ [Coercive estimates and separability theorems for elliptic equations in $R^{n}$. Trudy Matematicheskoho instituta AN SSSR - Proceedings of the Mathematical Institute of the USSR Academy of Sciences, 161, 195-217 [in Russian].

12 Muratbekov, M., \& Otelbaev, M. (2016). On the existence of a resolvent and separability for a class of singular hyperbolic type differential operators on an unbounded domain. Eurasian mathematical journal, 7(1), 50-67.

13 Akhiezer, N.I., \& Glazman, I.M. (1966). Teoriia lineinykh operatorov v hilbertovom prostranstve [Theory of linear operators in Hilbert space]. Moscow: Nauka [in Russian]. 


\author{
M.I. Tleubergenov ${ }^{1,2}$, G.K. Vassilina ${ }^{1,3, *}$, G.A. Tuzelbaeva ${ }^{1,2}$ \\ ${ }^{1}$ Institute of Mathematics and Mathematical Modeling, Almaty, Kazakhstan; \\ ${ }^{2}$ Al-Farabi Kazakh National University, Almaty, Kazakhstan; \\ ${ }^{3}$ Almaty University of Power Engineering and Telecommunications named after G.Daukeev, Almaty, Kazakhstan \\ (E-mail: marat207@mail.ru,v_gulmira@mail.ru,tuzelbayeva.gainizhamal@mail.ru)
}

\title{
On construction of a field of forces along given trajectories in the presence of random perturbations
}

\begin{abstract}
In this paper, a force field is constructed along a given integral manifold in the presence of random perturbing forces. In this case, two types of integral manifolds are considered separately: 1) trajectories that depend on generalized coordinates and do not depend on generalized velocities, and 2) trajectories that depend on both generalized coordinates and generalized velocities. The construction of the force field is carried out in the class of second-order stochastic Ito differential equations. It is assumed that the functions in the right-hand sides of the equation must be continuous in time and satisfy the Lipschitz condition in generalized coordinates and generalized velocities. Also this functions satisfy the condition for linear growth in generalized coordinates and generalized velocities.These assumptions ensure the existence and uniqueness up to stochastic equivalence of the solution to the Cauchy problem of the constructed equations in the phase space, which is a strictly Markov process continuous with probability 1 . To solve the two posed problems, stochastic differential equations of perturbed motion with respect to the integral manifold are constructed. Moreover, in the case when the trajectories depend on generalized coordinates and do not depend on generalized velocities, the second order equations of perturbed motion are constructed, and in the case when the trajectories depend on both generalized coordinates and generalized velocities, the first order equations of perturbed motion are constructed. And further, in both cases by Erugin's method necessary and sufficient conditions for solving the posed problems are derived.
\end{abstract}

Keywords: stochastic differential equations, inverse problems, stability, integral manifold.

\section{Introduction}

The theory of inverse problems of differential systems in the class of ordinary differential equations is quite fully developed in [1-6, etc.]. And set of ordinary differential equations is constructed along a given integral curve in [1]. This work later turned out to be fundamental in the formation and development of the theory of inverse problems of the dynamics of systems described by ordinary differential equations. Formulations, classification of inverse problems of differential systems are stated and general methods of their solving in the class of ordinary differential equations are developed in [2-6]. We also note [7-9], in which inverse problems of dynamics of automatic control systems are considered in the class of ordinary differential equations. Methods for solving inverse problems in the class of ordinary differential equations are generalized to the class of Ito stochastic differential equations in [10-14]. In this paper, the results of [15, 16], obtained in the class of ordinary differential equations, are extended to the class of Ito stochastic differential equations.

1 The problem of construction of a force field along given trajectories

(independent of velocities) in the presence of random perturbations

Let the trajectory

$$
\Lambda: \lambda(x, y, t)=0, \text { где } \lambda=\lambda(x, y, t) \in C_{x y t}^{222}, \lambda \in R^{1}
$$

be given. It is required to construct a force field in the presence of random perturbing forces so that the constructed force field has a given trajectory as an integral manifold

$$
\left\{\begin{array}{l}
\ddot{x}=X_{1}(x, y, t)+\widehat{\sigma}_{1}(x, y, t) \dot{\xi}, \\
\ddot{y}=Y_{1}(x, y, t)+\widetilde{\sigma}_{1}(x, y, t) \dot{\xi},
\end{array}\right.
$$

\footnotetext{
${ }^{*}$ Corresponding author.

E-mail:v_gulmira@mail.ru
} 
here $\xi=\xi(t, \omega)$ is random process with independent increments, which, following [17], can be represented as a sum $\xi=\xi_{0}+\int c(\mu) P^{0}(t, d \mu)$, where $\xi_{0}$ is a Wiener process and $P^{0}$ is a Poisson process. $P^{0}(t, d \mu)$ is the number of jumps of $P^{0}$ on the interval $[0, t]$, that fall on the set $d \mu$. $c(\mu)$ is a scalar function mapping the space $R^{2}$ into the space $R^{1}$ of values of the process $\xi(t)$ for any $t$.

Definition 1. A function $f(z, t)$ belongs to the class $K, f \in K$, if $z$ is continuous in $t, t \in[0, \infty]$, and is Lipschitz continuous in $x$ and $y \| f(z, t)-f\left(\widetilde{z}, t\|\leq B\| z-\widetilde{z} \|\right.$ in the entire space $z=(x, y)^{T} \in R^{2}$ and satisfies the condition $\|f(z, t)\| \leq B(1+\|z\|)$ of linear growth with respect to $z$ with some constant $B$.

It is assumed that, $X_{1}(x, y, t), Y_{1}(x, y, t), \widehat{\sigma}_{1}(x, y, t)$ and $\widetilde{\sigma}_{1}(x, y, t)$, belong to the class $K$, which ensures the existence and uniqueness in the space $R^{4}$ up to stochastic equivalence of solution $(x(t), y(t), \dot{x}(t), \dot{y}(t))^{T}$ of the system of equations (1.1) with the initial condition $\left(x\left(t_{0}\right), y\left(t_{0}\right), \dot{x}\left(t_{0}\right), \dot{y}\left(t_{0}\right)\right)^{T}=\left(x_{0}, y_{0}, \dot{x}_{0}, \dot{y}_{0}\right)^{T}$ which is continuous with probability 1 strictly Markov process [17].

The projections of the velocity of a material point $\dot{x}, \dot{y}$ onto the coordinate axes $x, y$ are determined from the equation

$$
\dot{\lambda}=\lambda_{t}+\lambda_{x} \dot{x}+\lambda_{y} \dot{y}
$$

Differentiating the last expression with respect to time, we obtain

$$
\begin{gathered}
\ddot{\lambda}=\lambda_{t t}+\left(\lambda_{t x}+\lambda_{x x} \dot{x}+\lambda_{x y} \dot{y}\right) \dot{x}+\lambda_{x} \ddot{x}+\left(\lambda_{t y}+\lambda_{x y} \dot{x}+\lambda_{y y} \dot{y}\right) \dot{y}+\lambda_{y} \ddot{y}= \\
=\lambda_{t t}+\lambda_{t x} \dot{x}+\lambda_{t y} \dot{y}+\lambda_{x x} \dot{x}^{2}+2 \lambda_{x y} \dot{x} \dot{y}+\lambda_{x}\left(X+\widehat{\sigma}_{1} \dot{\xi}\right)+\lambda_{y y} \dot{y}^{2}+\lambda_{y}\left(Y+\widetilde{\sigma}_{1} \dot{\xi}\right) .
\end{gathered}
$$

To ensure the integrality of set (1.1) for the system of differential equations (1.2), following Erugin's method, we introduce the vector function $A$ and the matrix $B$

$$
A_{1}=A_{1}(\lambda, \dot{\lambda} ; x, y, \dot{x}, \dot{y}, t), \quad B_{1}=B_{1}(\lambda, \dot{\lambda} ; x, y, \dot{x}, \dot{y}, t)
$$

with properties $A_{1}(0,0 ; x, y, \dot{x}, \dot{y}, t) \equiv B_{1}(0,0 ; x, y, \dot{x}, \dot{y}, t) \equiv 0$, such that

$$
\ddot{\lambda}=A_{1}+B_{1} \dot{\xi} .
$$

In view of (1.3) and (1.4), we have

$$
\begin{gathered}
A_{1}=\lambda_{t t}+\lambda_{t x} \dot{x}+\lambda_{t y} \dot{y}+\lambda_{x x} \dot{x}^{2}+2 \lambda_{x y} \dot{x} \dot{y}+\lambda_{y y} \dot{y}^{2}+\lambda_{x} X+\lambda_{y} Y, \\
B_{1}=\lambda_{x} \widehat{\sigma}_{1}+\lambda_{y} \widetilde{\sigma}_{1} .
\end{gathered}
$$

Let $\lambda_{x}^{2}+\lambda_{y}^{2} \neq 0$ takes place, then

a) if $\lambda_{x} \neq 0$ for any $x, y$, then

$$
\left\{\begin{array}{l}
X_{1}=\lambda_{x}^{-1}\left(A_{1}-\lambda_{x x} \dot{x}^{2}-2 \lambda_{x y} \dot{x} \dot{y}-\lambda_{y y} \dot{y}^{2}-\lambda_{y} Y\right) \\
\widehat{\sigma}_{1}=\lambda_{x}^{-1}\left(B_{1}-\lambda_{y} \widetilde{\sigma}_{1}\right)
\end{array}\right.
$$

for any $Y_{1}, \widetilde{\sigma}_{1}$ from the class $K$;

б) if $\lambda_{y} \neq 0$ for any $x, y$, then

$$
\left\{\begin{array}{l}
Y_{1}=\lambda_{y}^{-1}\left(A_{1}-\lambda_{x x} \dot{x}^{2}-2 \lambda_{x y} \dot{x} \dot{y}-\lambda_{y y} \dot{y}^{2}-\lambda_{x} X\right) \\
\widetilde{\sigma}_{1}=\lambda_{y}^{-1}\left(B_{1}-\lambda_{x} \widehat{\sigma}_{1}\right)
\end{array}\right.
$$

for any $X_{1}, \widehat{\sigma}_{1}$ from the class $K$.

Theorem 1. A necessary and sufficient condition that the set of force fields (1.2) has a given trajectory (1.1) in the presence of random perturbations from the class of processes with independent increments is that one of conditions (1.5) or (1.6) be satisfied.

\section{The problem of construction of a force field along given trajectories (depending on velocities) in the presence of random perturbations}

Let us consider the case when the given trajectory $\lambda$ depends on both generalized coordinates and generalized velocities

$$
\Lambda: \lambda(x, \dot{x}, y, \dot{y}, t)=0, \text { where } \lambda=\lambda(x, \dot{x}, y, \dot{y}, t) \in C_{x \dot{x} y \dot{y} t}^{12121}, \lambda \in R^{1} .
$$


It is required to construct a force field in the presence of random perturbing forces so that the constructed force field has a given trajectory as an integral manifold

$$
\left\{\begin{array}{l}
\ddot{x}=X_{2}(x, y)+\widehat{\sigma}_{2}(x, y) \dot{\eta}, \\
\ddot{y}=Y_{2}(x, y, t)+\widetilde{\sigma}_{2}(x, y, t) \dot{\eta},
\end{array}\right.
$$

here $\eta=\eta(t, \omega)$ is scalar Wiener process [17].

Let us compose the equation of the perturbed motion relative to a given trajectory (2.1). To do this, we differentiate (2.1) with respect to time and obtain

$$
\begin{gathered}
\dot{\lambda}=\lambda_{t}+\lambda_{x} \dot{x}+\lambda_{\dot{x}} \ddot{x}+\lambda_{y} \dot{y}+\lambda_{\dot{y}} \ddot{y}= \\
=\lambda_{x} \dot{x}+\lambda_{\dot{x}}\left(X_{2}+\widehat{\sigma}_{2} \dot{\eta}\right)+\lambda_{y} \dot{y}+\lambda_{\dot{y}}\left(Y_{2}+\widetilde{\sigma}_{2} \dot{\eta}\right)+\frac{1}{2}\left(\lambda_{\dot{x} \dot{x}} \widehat{\sigma}_{2}^{2}+\lambda_{\dot{y} \dot{y}} \widetilde{\sigma}_{2}^{2}\right) .
\end{gathered}
$$

Further, following Erugin's method [1], we introduce the vector function $A_{2}$ the matrix $B_{2}$

$$
A_{2}=A_{2}(\lambda ; x, y, \dot{x}, \dot{y}), B_{2}=B_{2}(\lambda ; x, y, \dot{x}, \dot{y})
$$

with properties $A_{2}(0 ; x, y, \dot{x}, \dot{y})=0, B_{2}(0 ; x, y, \dot{x}, \dot{y})=0$ such that

$$
\dot{\lambda}=A_{2}+B_{2} \dot{\eta} .
$$

In view of (2.3) and (2.4), we arrive at the relations

$$
\begin{gathered}
A_{2}=\lambda_{x} \dot{x}+\lambda_{\dot{x}} X+\lambda_{y} \dot{y}+\lambda_{\dot{y}} Y+\frac{1}{2}\left(\lambda_{\dot{x} \dot{x}} \widehat{\sigma}_{2}^{2}+\lambda_{\dot{y} \dot{y}} \widetilde{\sigma}_{2}^{2}\right), \\
B_{2}=\lambda_{\dot{x}} \widehat{\sigma}_{2}+\lambda_{\dot{y}} \widetilde{\sigma}_{2} .
\end{gathered}
$$

Let $\lambda_{\dot{x}}^{2}+\lambda_{\dot{y}}^{2} \neq 0$ takes place. Then

a) if $\lambda_{\dot{x}} \neq 0$ for any $x, y$, then

$$
\left\{\begin{array}{l}
X_{2}=\lambda_{\dot{x}}^{-1}\left(A_{2}-\lambda_{x} \dot{x}-\lambda_{y} \dot{y}+\lambda_{\dot{y}} Y_{2}-\frac{1}{2}\left(\lambda_{\dot{x} \dot{x}} \widehat{\sigma}_{2}^{2}+\lambda_{\dot{y} \dot{y}} \widetilde{\sigma}_{2}^{2}\right)\right) \\
\widehat{\sigma}_{2}=\lambda_{\dot{x}}^{-1}\left(B_{2}-\lambda_{\dot{y}} \widetilde{\sigma}_{2}\right)
\end{array}\right.
$$

for any $Y_{2}, \widetilde{\sigma}_{2}$ from $K$;

b) if $\lambda_{\dot{y}} \neq 0$ for any $x, y$, then

$$
\left\{\begin{array}{l}
Y_{2}=\lambda_{\dot{y}}^{-1}\left(A_{2}-\lambda_{x} \dot{x}-\lambda_{\dot{x}} X_{2}+\lambda_{y} \dot{y}-\frac{1}{2}\left(\lambda_{\dot{x} \dot{x}} \widehat{\sigma}_{2}^{2}+\lambda_{\dot{y} \dot{y}} \widetilde{\sigma}_{2}^{2}\right)\right) \\
\widetilde{\sigma}_{2}=\lambda_{\dot{y}}^{-1}\left(B_{2}-\lambda_{\dot{x}} \widehat{\sigma}_{1}\right)
\end{array}\right.
$$

for any $X_{2}, \widehat{\sigma}_{2}$ from the class $K$.

The following theorem holds.

Theorem 2. A necessary and sufficient condition that the set of force fields (2.2) has a given trajectory (2.1) in the presence of random perturbations from the class of Wiener processes is that one of conditions (2.5) or (2.6) be satisfied.

\section{Conclusion}

Thus, the article deals with stochastic problems of constructing a force field along given trajectories. In the first section trajectories depend on generalized coordinates and do not depend on generalized velocities. And in the second section trajectories depend on both generalized coordinates and generalized velocities. The obtained results extend Galiullin's some statements [2,3] on the construction of a force field from a given family of trajectories in the class of ordinary differential equations to the class of second-order stochastic Ito differential equations. 


\title{
Acknowledgments
}

This research is funded by the Science Committee of the Ministry of Education and Science of the Republic of Kazakhstan (Grant No. AP08955847).

\author{
References
}

1 Еругин Н.П. Построение всего множества систем дифференциальных уравнений, имеющих заданную интегральную кривую / Н.П. Еругин // Прикладная математика и механика. - 1952. - Т. 10. - Вып. 6. - С. 659-670.

2 Галиуллин А.С. Методы решения обратных задач динамики / А.С. Галиуллин. - М.: РУДН, 1986. $-224 \mathrm{c}$.

3 Галиуллин А.С. Избранные труды: [В 2-х т.]. - Т. ІІ / А.С. Галиуллин. - М.: РУДН, 2009. - 462 с.

4 Мухаметзянов И.А. Уравнения программных движений / И.А. Мухаметзянов, Р.Г. Мухарлямов. М.: РУДН, 1986. -88 c.

5 Мухарлямов Р.Г. О построении систем дифференциальных уравнений движения механических систем / Р.Г. Мухарлямов // Дифференциальные уравнения. - 2003. - Т. 39. - № 3. - С. 343-353.

6 Мухарлямов Р.Г. Моделирование процессов управления, устойчивость и стабилизация систем с программными связями / Р.Г. Мухарлямов // Изв. РАН. Теория и системы управления. -2015 . - № 1 . - C. $15-28$.

7 Zhumatov S.S. Asymptotic Stability of Implicit Differential Systems in the Vicinity of Program Manifold / S.S. Zhumatov // Ukrainian Mathematical Journal. - 2014. - Vol. 66(4). - P. 625-632. DOI: 10.1007/s11253-014-0959-y.

8 Zhumatov S.S. Exponential Stability of a Program Manifold of Indirect Control Systems / S.S. Zhumatov // Ukrainian Mathematical Journal. -2010. - Vol. 62(6). - P. 907-915. DOI: 10.1007/s11253-010-0399-2.

9 Zhumatov S.S. Stability of a Program Manifold of Control Systems with Locally Quadratic Relations / S.S. Zhumatov // Ukrainian Mathematical Journal. - 2009. - Vol. 61(3). - P. 500-509. DOI: 10.1007/s11253-009-0224-y.

10 Tleubergenov M.I. On the Solvability of the Main Inverse Problem for Stochastic Differential Systems / M.I. Tleubergenov, G.T. Ibraeva // Ukrainian Mathematical Journal. - 2019. - Vol. 71. - No.1. P. 157-165.

11 Tleubergenov M.I. Stochastic Inverse Problem with Indirect Control / M.I. Tleubergenov, G.T. Ibraeva // Differential equations. - 2017. - Vol. 53. - Issue 10. - P. 1387-1391.

12 Tleubergenov M.I. On Construction of Force Function in the Presence of Random Perturbations / M.I. Tleubergenov, D.T. Azhymbaev // Springer Proceedings in Mathematics and Statistics. - Springer, 2017. - Vol. 216. - P. 443-450.

13 Tleubergenov M.I. On the Restoration Problem with Degenerated Diffusion/M.I. Tleubergenov, G.T. Ibraeva // TWMS journal of pure and applied mathematics. - 2015. - Vol.6. - Issue 1. - P. 93-99.

14 Ibraeva G.T. Main Inverse Problem for Differential Systems With Degenerate Diffusion / G.T. Ibraeva, M.I. Tleubergenov // Ukrainian Mathematical Journal. — 2013. - Vol. 65. — № 5. - P. 787-792.

15 Галиуллин А.С. Построение поля сил по заданным траекториям / А.С. Галиуллин // Сб. науч.метод. ст. - Вып. 10. - М.: Высш. шк., 1980. - С. 31-34.

16 Галиуллин А.С. Построение поля сил по заданному семейству траекторий / А.С. Галиуллин // Дифференциальные уравнения. - 1981. - Т. 17, № 8. - С. 1487-1489.

17 Пугачев В.С. Стохастические дифференциальные системы. Анализ и фильтрация / В.С. Пугачев, И.Н. Синицын. - М.: Наука, 1990. - 632 с. 
М.Ы. Тілеубергенов, Г.Қ. Василина, Г.А. Түзелбаева

\title{
Кездейсоқ түрткі болғанда берілген траекториялар бойынша күштер өрісін тұрғызу туралы
}

\begin{abstract}
Мақалада кездейсоқ түрткілеуші күштер болғанда берілген интегралдық көпбейне бойынша күштік өріс тұрғызылған. Бұл арада интегралдық көпбейненің екі түрі жеке қарастырылды: 1) жалпыланған координаттарға тәуелді және жалпыланған жылдамдықтардан тәуелсіз емес траекториялар және 2) жалпыланған координаттарға да жалпыланған жылдамдыққа да тәуелді траекториялар. Күштік өрісті тұрғызу екінші ретті Ито стохастикалық дифференциалдық теңдеулер класында жүргізіледі. Бұл арада, теңдеудің оң жағына кіретін функциялар, уақыт бойынша үзіліссіз және де жалпыланған координаттар және жалпыланған жылдамдықтар бойынша Липшиц шартын қанағаттандыратын, сонымен бірге жалпыланған координаттар және жалпыланған жылдамдықтар бойынша сызықтық өсуді қанағаттандыратын болуы болжамдалды. Бұл болжамдар фазалық кеңістікте, тұрғызылған теңдеулердің Коши есебінің шешімінің, үзіліссіз 1 ықтималдықты қатаң марковтік үдеріс болып табылатын, стохастикалық эквиваленттікке дейінгі бар болуын және жалқылығын қамтамасыз етеді. Қойылған екі есепті шешу үшін түрткіленген қозғалыстың стохастикалық дифференциалдық теңдеулері интегралдық көпбейне бойынша тұрғызылды. Жалпыланған координаттарға тәуелді және жалпыланған жылдамдықтардан тәуелсіз траекториялар жағдайында, екінші ретті түрткіленген қозғалыс теңдеулері; ал жалпыланған координаттарға да жалпыланған жылдамдықтарға да тәуелді траекториялар жағдайында, бірінші ретті түрткіленген қозғалыс теңдеулері тұрғызылады. Әрі қарай, Еругин әдісі бойынша екі жағдайда да қойылған есептердің шешілуінің қажетті және жеткілікті шарттары қорытылып шығарылды.
\end{abstract}

Kiлm сөздер: стохастикалық дифференциалдық теңдеулер, кері есептер, орнықтылық, интегралдық көпбейне.

\author{
М.И. Тлеубергенов, Г.К. Василина, Г.А. Тузелбаева
}

\section{О построении поля сил по заданным траекториям при наличии случайных возмущений}

В статье построено силовое поле по заданному интегральному многообразию при наличии случайных возмущающих сил. При этом отдельно рассмотрены два вида интегральных многообразий: 1) траектории, зависящие от обобщенных координат и не зависящие от обобщенных скоростей, и 2) траектории, зависящие как от обобщенных координат, так и от обобщенных скоростей. Построение силового поля проводится в классе стохастических дифференциальных уравнений Ито второго порядка. При этом предполагается, что функции, входящие в правые части уравнения, должны быть непрерывными по времени и удовлетворять условию Липшица по обобщенным координатам и обобщенным скоростям, а также условию линейного роста по обобщенным координатам и обобщенным скоростям. Эти предположения обеспечивают в фазовом пространстве существование и единственность до стохастической эквивалентности решения задачи Коши построенных уравнений, являющегося непрерывным с вероятностью 1 строго марковским процессом. Для решения поставленных двух задач строятся стохастические дифференциальные уравнения возмущенного движения относительно интегрального многообразия. Причем, в случае, когда траектории зависят от обобщенных координат и не зависят от обобщенных скоростей, строятся уравнения возмущенного движения второго порядка, а в случае, когда траектории зависят как от обобщенных координат, так и от обобщенных скоростей, строятся уравнения возмущенного движения первого порядка. И далее, методом Еругина в обоих случаях выводятся необходимые и достаточные условия решения поставленных задач.

Ключевые слова: стохастические дифференциальные уравнения, обратные задачи, устойчивость, интегральное многообразие. 


\section{References}

1 Erugin, N.P. (1952). Postroenie vseho mnozhestva sistem differentsialnykh uravnenii, imeiushikh zadannuiu intehralnuiu krivuiu [Construction of the entire set of systems of differential equations with a given integral curve]. Prikladnaia matematika i mekhanika - Applied Mathematics and Mechanics, 10(6), 659670 [in Russian].

2 Galiullin, A.S. (1986). Metody resheniia obratnykh zadach dinamiki [Methods for solving inverse problems of dynamics]. Moscow: RUDN [in Russian].

3 Galiullin, A.S. (2009). Izbrannye trudy [v dvukh tomakh] [Selected works in two volumes. Vol. II]. Moscow: RUDN [in Russian].

4 Mukhametzyanov, I.A., \& Mukharlyamov, R.G. (1986). Uravneniia prohrammnykh dvizhenii [Equations of program motions]. Moscow: RUDN [in Russian].

5 Mukharlyamov, R.G. (2003). O postroienii sistem differentsialnykh uravnenii dvizheniia mekhanicheskikh sistem [On the construction of systems of differential equations of motion of mechanical systems]. $D i$ fferentsialnye uravneniia - Differential Equations, 39(3), 343-353 [in Russian].

6 Mukharlyamov, R.G. (2015). Modelirovanie protsessov upravleniia, ustoichivost i stabilizatsiia sistem s prohrammnymi sviaziami [Modeling of control processes, stability and stabilization of systems with programmed connections]. Izvestiia RAN. Teoriia i sistemy upravleniia - Izvestiia RAN. Control theory and systems, 1, 15-28 [in Russian].

7 Zhumatov, S.S. (2014). Asymptotic Stability of Implicit Differential Systems in the Vicinity of Program Manifold. Ukrainian Mathematical Journal, 66(4), 625-632. DOI: 10.1007/s11253-014-0959-y.

8 Zhumatov, S.S. (2010). Exponential Stability of a Program Manifold of Indirect Control Systems. Ukrainian Mathematical Journal, 62(6), 907-915. DOI: 10.1007/s11253-010-0399-2.

9 Zhumatov, S.S. (2009). Stability of a Program Manifold of Control Systems with Locally Quadratic Relations. Ukrainian Mathematical Journal, 61(3), 500-509. DOI: 10.1007/s11253-009-0224-y.

10 Tleubergenov, M.I., \& Ibraeva, G.T. (2019). On the Solvability of the Main Inverse Problem for Stochastic Differential Systems. Ukrainian Mathematical Journal, 71(1), 157-165.

11 Tleubergenov, M.I., \& Ibraeva, G.T. (2017). Stochastic Inverse Problem with Indirect Control. Differential equations, 53(10), 1387-1391.

12 Tleubergenov, M.I., \& Azhymbaev, D.T. (2017). On Construction of Force Function in the Presence of Random Perturbations. Springer Proceedings in Mathematics and Statistics, Springer, 216, 443-450.

13 Tleubergenov, M.I., \& Ibraeva, G.T. (2015). On the Restoration Problem with Degenerated Diffusion. TWMS journal of pure and applied mathematics, 6(1), 93-99.

14 Ibraeva, G.T., \& Tleubergenov, M.I. (2013). Main Inverse Problem for Differential Systems With Degenerate Diffusion. Ukrainian Mathematical Journal, 65(5), 787-792.

15 Galiullin, A.S. (1980). Postroenie polia sil po zadannym traektoriiam [Construction of the field of forces along the specified trajectories]. Sbornik nauchno-metodicheskikh statei-Collection of scientific and methodological articles, 10, 31-34 [in Russian].

16 Galiullin, A.S. (1981). Postroenie polia sil po zadannomu semeistvu traiektorii [Construction of a force field for a given set of trajectories]. Differentsialnye uravneniia - Differential Equations, 17(8), 1487-1489 [in Russian].

17 Pugachev, V.S., \& Sinitsyn, I.N. (1990). Stokhasticheskie differentsialnye sistemy. Analiz $i$ filtratsiia [Stochastic differential systems. Analysis and filtering]. Moscow: Nauka [in Russian]. 


\author{
A.N. Yesbayev*, M.N.Ospanov \\ L.N.Gumilyov Eurasian National University, Nur-Sultan, Kazakhstan \\ (E-mail: adilet.e@gmail.com,myrzan66@mail.ru)
}

\title{
The solvability conditions for the second order nonlinear differential equation with unbounded coefficients in $L_{2}(\mathbb{R})$
}

\begin{abstract}
The article deals with the existence of a generalized solution for the second order nonlinear differential equation in an unbounded domain. Intermediate and lower coefficients of the equation depends on the required function and considered smooth. The novelty of the work is that we prove the solvability of a nonlinear singular equation with the leading coefficient not separated from zero. In contrast to the works considered earlier, the leading coefficient of the equation can tend to zero, while the intermediate coefficient tends to infinity and does not depend on the growth of the lower coefficient. The result obtained formulated in terms of the coefficients of the equation themselves; there are no conditions on any derivatives of these coefficients.
\end{abstract}

Keywords: second order differential equation, nonlinear differential equation, differential equation in an unbounded domain, generalized solution, solvability.

\section{Introduction}

We investigate the following second-order singular differential equation

$$
-\rho(x)\left(\rho(x) y^{\prime}\right)^{\prime}+r(x, y) y^{\prime}+s(x, y) y=f(x)
$$

where $x \in \mathbb{R}=(-\infty,+\infty), \rho$ is a twice continuously differentiable function, $r$ is a continuously differentiable function, and $s$ is a continuous function, $f \in L_{2} \stackrel{\text { def }}{=} L_{2}(\mathbb{R}),\|\cdot\|_{2}$ is the norm in $L_{2}$. The singularity of the equation (1) means that it is given in a non-compact domain, and its coefficients can be unbounded.

The study of the equation (1) and its multidimensional generalizations is related to applications in quantum mechanics, stochastic analysis and stochastic differential equations [1-4]. In the above references the linear case is considered and results are obtained for $s(x, y)=s(x)>\delta>0$, and the growth of $|r(x, y)|=|r(x)|$ at infinity is bounded by some positive power of $s(x)$. In the following researches [5-8] the linear case of equation (1) is also considered and it is assumed that the intermediate coefficient $r(x)$ can not grow faster than $|x| \ln |x|$ at infinity. In [5-8] issues on solvability of the equation (1) were considered only for the case $\rho(x) \geqslant \delta>0$. The issue on solvability of the equation (1) stays unresolved for the case when the growth of $|r(x)|$ is faster than $|x| \ln |x|$ and is not dependent on $s$, and also when the coefficient $\rho(x)$ approaches zero as $x \rightarrow+\infty$ or as $x \rightarrow-\infty$.

For the case when $\rho \equiv 1$ and $|r|$ grows rapidly and does not depend on the coefficient $s$ the equation (1) was analyzed in [9]. Here it was determined the solvability and the maximal regularity for the solution. The linear case for the equation (1) with a fast-growing growing intermediate coefficient was studied in [10] (when $f \in L_{2}$ ), [11] (when $f \in L_{1}(\mathbb{R})$ ) and [12] (when $f \in L_{p}(\mathbb{R}), 1<p<+\infty$ ). In [10-12] the function $\rho(x)$ is assumed to be separated from zero and bounded, or equal to 1 . The study of the solvability of different classes of partial differential equations with unbounded coefficients is presented in [13-16].

Note that the rapid and independent growth of the absolute value of the intermediate coefficient $r$ makes a big difference for solvability of the equation (1). Firstly, in this case the coefficient $s$ can be unbounded from below. Moreover it can approach to $-\infty$ with certain rate [11, 12], where the rate of approaching $s$ to $-\infty$ depends on the growth rate of $|r|$. Also let us note that in the study of the Sturm-Liouville equation (the case $\rho \equiv 1, r \equiv 0, s(x, y)=s(x))$ it is usually assumed that $s \geqslant-k x^{2}$ for some $k$ [2]. Such condition in the case of equation (1) with unbounded $r$ is not necessary.

\footnotetext{
${ }^{*}$ Corresponding author.

E-mail: adilet.e@gmail.com
} 
Secondly, due to the growth of the absolute value of $r$ in the equation (1) it turns out we can assume approaching zero at infinity for the coefficient $\rho$ in the leading term, thereby considering the so-called case of degeneration. The theorem 1 presented below shows that the rate of approaching $\rho$ to zero also depends on the growth of $|r|$.

In the work [17] the results of the correct solvability and also a coercive estimate for the equation (1) was established in the case $r(x, y)=r(x), s(x, y)=s(x)$ and $\rho(x)>0$. In this paper we propose to extend some of the results obtained in [17] to the case of nonlinear generalization of equation (1).

\section{Preliminaries}

Let $C_{0}^{(k)}(\mathbb{R})(k=1,2, \ldots)$ be the set of $k$ times continuously differentiable functions on $\mathbb{R}$ with compact support and $C_{\text {loc }}^{(j)}(\mathbb{R}) \stackrel{\text { def }}{=}\left\{y: \psi y \in C_{0}^{(j)}(\mathbb{R}), \forall \psi \in C_{0}^{(j)}(\mathbb{R})\right\}(j=1,2)$. Consider the following linear equation

$$
-\rho(x)\left(\rho(x) y^{\prime}\right)^{\prime}+r(x) y^{\prime}+s(x) y=F(x) .
$$

Let $g$ and $h \neq 0$ be given continuous functions. We denote

$$
\begin{gathered}
\alpha_{g, h}(t) \stackrel{\text { def }}{=}\|g\|_{L_{2}(0, t)}\left\|h^{-1}\right\|_{L_{2}(t,+\infty)} \quad(t>0), \quad \beta_{g, h}(\tau) \stackrel{\text { def }}{=}\|g\|_{L_{2}(\tau, 0)}\left\|h^{-1}\right\|_{L_{2}(-\infty, \tau)} \quad(\tau<0), \\
\alpha_{g, h} \stackrel{\text { def }}{=} \sup _{t>0} \alpha_{g, h}(t), \beta_{g, h} \stackrel{\text { def }}{=} \sup _{\tau<0} \beta_{g, h}(\tau), \gamma_{g, h} \stackrel{\text { def }}{=} \max \left(\alpha_{g, h}, \beta_{g, h}\right) .
\end{gathered}
$$

The following statement is proved in [9].

Lemma 1. If $g$ and $h$ are continuous functions such that $\gamma_{g, h}<+\infty$. Then for $y \in C_{0}^{(1)}(\mathbb{R})$ the following inequality holds

$$
\int_{-\infty}^{+\infty}|g(x) y(x)|^{2} d x \leqslant C_{1} \int_{-\infty}^{+\infty}\left|h(x) y^{\prime}(x)\right|^{2} d x .
$$

Moreover we have $\left(\min \left(\alpha_{g, h}, \beta_{g, h}\right)\right)^{2} \leqslant C_{1} \leqslant 4\left(\gamma_{g, h}\right)^{2}$.

Let the operator $l_{0} y=-\rho(x)\left(\rho(x) y^{\prime}\right)^{\prime}+r(x) y^{\prime}+s(x) y$ is defined on the set $C_{0}^{(2)}(\mathbb{R})$, we denote the closure of the operator $l_{0}$ by $l$ in $L_{2}$. The function $y \in D(l)$ such that $l y=f$ is said to be a solution of the equation (2).

The following statement is proved in [17].

Lemma 2. If $0<\rho(x)<+\infty$ is a twice continuously differentiable function, $r(x) \geqslant 1$ is a continuously differentiable function, and $s(x)$ is a continuous function, $r(x) \geqslant \rho^{2}(x), \gamma_{1, \sqrt{r}}<+\infty, \gamma_{s, r}<+\infty$ and there exists $a \in \mathbb{R}$ such that

$$
\sup _{x<a}\left\{\rho(x) \exp \left(-\int_{x}^{a} \frac{r(t)}{\rho^{2}(t)} d t\right)\right\}<+\infty .
$$

Next, let there be $C_{2}>1$ such that

$$
C_{2}^{-1} \leqslant \frac{\rho(x)}{\rho(\nu)} \leqslant C_{2}, \quad C_{2}^{-1} \leqslant \frac{r(x)}{r(\nu)} \leqslant C_{2}, \quad \text { as }|x-\nu| \leqslant 1 .
$$

Then for any right-hand side $F \in L_{2}$ the linear equation (2) has a unique solution $y$ and for $y$ the following inequality holds

$$
\left\|-\rho\left(\rho y^{\prime}\right)^{\prime}\right\|_{2}+\left\|r y^{\prime}\right\|_{2}+\|s y\|_{2} \leqslant C_{3}\|F\|_{2},
$$

where $C_{3}$ depends only on $C_{2}, \gamma_{1, \sqrt{r}}$ and $\gamma_{s, r}$.

The solvability conditions for the second order non-linear differential equation

For continuous functions of two variables $g(x, y)$ and $h(x, y) \neq 0$ we denote

$$
\alpha_{g, h}(t, y) \stackrel{\text { def }}{=}\left(\int_{0}^{t}|g(x, y)|^{2} d x\right)^{\frac{1}{2}}\left(\int_{t}^{+\infty} \frac{d x}{|h(x, y)|^{2}}\right)^{\frac{1}{2}} \quad(t>0)
$$




$$
\begin{gathered}
\beta_{g, h}(\tau, y) \stackrel{\text { def }}{=}\left(\int_{\tau}^{0}|g(x, y)|^{2} d x\right)^{\frac{1}{2}}\left(\int_{-\infty}^{\tau} \frac{d x}{|h(x, y)|^{2}}\right)^{\frac{1}{2}}(\tau<0), \\
\alpha_{g, h}(y) \stackrel{\text { def }}{=} \sup _{t>0} \alpha_{g, h}(t, y), \beta_{g, h}(y) \stackrel{\text { def }}{=} \sup _{\tau<0} \beta_{g, h}(\tau, y), \\
\gamma_{g, h}(y) \stackrel{\text { def }}{=} \max \left(\alpha_{g, h}(y), \beta_{g, h}(y)\right) .
\end{gathered}
$$

Definition 1. Let $y \in L_{2} . y$ is said to be a solution of the equation (1), if there exist a sequence $\left\{y_{n}\right\} \subset C_{\text {loc }}^{(2)}(\mathbb{R})$ such that

$$
\left\|\psi\left(y_{n}-y\right)\right\|_{2} \rightarrow 0 \text { and }\left\|\psi\left(L y_{n}-f\right)\right\|_{2} \rightarrow 0 \text { as } n \rightarrow+\infty, \quad \forall \psi \in C_{0}^{(\infty)}(\mathbb{R}) .
$$

Theorem 1. Let $\rho(x)$ be a twice continuous differentiable and bounded function, $r(x, t)$ be a continuous differentiable function, $s(x, t)$ be a continuous function and

$$
\inf _{t \in \mathbb{R}} r(x, t) \geqslant \rho^{2}(x), \quad \sup _{y \in \mathbb{R}} \gamma_{1, \sqrt{r(x, y)}}<+\infty, \quad \sup _{t \in \mathbb{R}} \gamma_{s(\cdot, t), r(\cdot, t)}<+\infty,
$$

there exists $a \in \mathbb{R}$ such that

$$
\sup _{x<a}\left\{\rho(x) \exp \left(-\int_{x}^{a} \frac{\inf _{t \in \mathbb{R}} r(v, t)}{\rho^{2}(v)} d v\right)\right\}<+\infty .
$$

Also for some $\delta>0$ and $\forall A>0$ the inequalities holds

$$
\begin{gathered}
r(x, y) \geqslant\left(1+x^{2}\right)^{\frac{3}{4}+\delta} \\
\sup _{|x-\nu| \leqslant 1\left|C^{\prime}-C^{\prime \prime}\right| \leqslant A} \frac{r\left(x, C^{\prime}\right)}{r\left(x, C^{\prime \prime}\right)} \leqslant T(A)<+\infty, \quad C_{4}^{-1} \leqslant \frac{\rho(x)}{\rho(\nu)} \leqslant C_{4}, \text { as }|x-\nu|<1 .
\end{gathered}
$$

Then the equation (1) have a solution $y$, and for $y$ the following inequality holds

$$
\left\|-\rho(x)\left(\rho(x) y^{\prime}\right)^{\prime}\right\|_{2}+\left\|r(x, y) y^{\prime}\right\|_{2}+\|s(x, y) y\|_{2}<+\infty .
$$

Proof. Let $C(\mathbb{R})$ be a space of continuous and bounded functions with the norm $\|y\|_{C(\mathbb{R})} \stackrel{\text { def }}{=} \sup _{t \in \mathbb{R}}|y(t)|$, and $\varepsilon$ and $A$ are given positive numbers. We consider the following set

$$
B_{A} \stackrel{\text { def }}{=}\left\{z \in C(\mathbb{R}):\|z\|_{C(\mathbb{R})} \leqslant A\right\} .
$$

Let $v \in B_{A}$, and $L_{v, \varepsilon}$ be a closure in $L_{2}$ of linear differential operator

$$
l_{v, \varepsilon}=-\rho(x)\left(\rho(x) y^{\prime}\right)^{\prime}+\left(r(x, v(x))+\varepsilon\left(1+x^{2}\right)\right) y^{\prime}+s(x, v(x)) y,
$$

defined on the set $C_{0}^{(2)}(\mathbb{R})$. Now we consider the equation

$$
L_{v, \varepsilon} y=f
$$

A function $y \in D\left(L_{v, \varepsilon}\right)$ satisfying the equation (4) we call a solution of that equation. Since the conditions of lemma 2 hold for the functions $\rho(x), r(x, v(x))+\varepsilon\left(1+x^{2}\right), s(x, v(x))$ then for any $f \in L_{2}$ the equation (4) has unique solution $y=y_{\varepsilon}(x)$, and for $y$ the following estimate holds

$$
\left\|-\rho(x)\left(\rho(x) y^{\prime}\right)^{\prime}\right\|_{2}+\left\|\left(r(x, v(x))+\varepsilon\left(1+x^{2}\right)\right) y^{\prime}\right\|_{2}+\|s(x, v(x)) y\|_{2} \leqslant C_{5}\|f\|_{2} .
$$


Let $k>0$. Using the Hölder's inequality we get

$$
\begin{aligned}
&\left|\left(1+x^{2}\right)^{k} y\right|=\int_{-\infty}^{x}\left(\left(1+t^{2}\right)^{k} y\right)^{\prime} d t \leqslant \int_{-\infty}^{x}\left(1+t^{2}\right)^{k}\left|y^{\prime}\right| d t+k \int_{-\infty}^{x}\left(1+t^{2}\right)^{k}|y| d t= \\
&=\int_{-\infty}^{x}\left(1+t^{2}\right)^{-\alpha}\left(1+t^{2}\right)^{k+\alpha}\left|y^{\prime}\right| d t+k \int_{-\infty}^{x}\left(1+t^{2}\right)^{-\beta}\left(1+t^{2}\right)^{k+\beta}|y| d t \leqslant \\
& \leqslant\left(\int_{-\infty}^{x}\left(1+t^{2}\right)^{-2 \alpha} d t\right)^{\frac{1}{2}}\left(\int_{-\infty}^{x}\left(1+t^{2}\right)^{2(k+\alpha)}\left|y^{\prime}\right|^{2} d t\right)^{\frac{1}{2}}+ \\
&+\left(\int_{-\infty}^{x}\left(1+t^{2}\right)^{-2 \beta} d t\right)^{\frac{1}{2}}\left(\int_{-\infty}^{x}\left(1+t^{2}\right)^{2(k+\beta)}|y|^{2} d t\right)^{\frac{1}{2}} .
\end{aligned}
$$

We choose the numbers $\alpha$ and $\beta$ so that $\frac{3}{4}<\alpha, \frac{1}{4}<\beta \leqslant \alpha-\frac{1}{2}$. Then

$$
\begin{aligned}
&\left(\int_{-\infty}^{x}\left(1+t^{2}\right)^{-2 \alpha} d t\right)^{\frac{1}{2}}\left(\int_{-\infty}^{x}\left(1+t^{2}\right)^{2(k+\alpha)}\left|y^{\prime}\right|^{2} d t\right)^{\frac{1}{2}}+ \\
&+\left(\int_{-\infty}^{x}\left(1+t^{2}\right)^{-2 \beta} d t\right)^{\frac{1}{2}}\left(\int_{-\infty}^{x}\left(1+t^{2}\right)^{2(k+\beta)}|y|^{2} d t\right)^{\frac{1}{2}} \leqslant \\
& \leqslant C_{6}\left\|\left(1+x^{2}\right)^{k+\alpha} y^{\prime}\right\|_{2}+C_{7}\left\|\left(1+x^{2}\right)^{k+\beta} y\right\|_{2} .
\end{aligned}
$$

Using the lemma 1 and the condition $\alpha \geqslant \beta+\frac{1}{2}$ we obtain

$$
C_{6}\left\|\left(1+x^{2}\right)^{k+\alpha} y^{\prime}\right\|_{2}+C_{7}\left\|\left(1+x^{2}\right)^{k+\beta} y\right\|_{2} \leqslant C_{8}\left\|\left(1+x^{2}\right)^{k+\alpha} y^{\prime}\right\|_{2} .
$$

Let $\alpha=\frac{3}{4}+\frac{\delta}{2}$ and $\beta=\frac{1}{4}+\frac{\delta}{2}$, where $\delta$ is the number from the condition (3). Then from (6) and (7) we receive

$$
\sup _{x \in \mathbb{R}}\left|\left(1+x^{2}\right)^{k} y\right| \leqslant C_{8}\left\|\left(1+x^{2}\right)^{k+\frac{3}{4}+\frac{\delta}{2}} y^{\prime}\right\|_{2} .
$$

Finally, by putting $k=\frac{\delta}{2}$ and taking into account the condition (3), we obtain the following estimate

$$
\sup _{x \in \mathbb{R}}\left|\left(1+x^{2}\right)^{\frac{\delta}{2}} y\right| \leqslant C_{8}\left\|r y^{\prime}\right\|_{2} .
$$

Therefore due to (5), lemma 1 and the condition (3) we have

$$
\begin{aligned}
\|y\|_{W} \stackrel{\text { def }}{=}\left\|-\rho(x)\left(\rho(x) y^{\prime}\right)^{\prime}\right\|_{2}+\left\|\left(r(x, v(x))+\left(1+x^{2}\right)\right) y^{\prime}\right\|_{2}+ \\
\quad+\left\|\left(s(x, v(x))+\left(1+x^{2}\right)^{\frac{1}{4}}\right) y\right\|_{2}+\sup _{x \in \mathbb{R}}\left|\left(1+x^{2}\right)^{\frac{\delta}{2}} y(x)\right| \leqslant C_{9}\|f\|_{2},
\end{aligned}
$$

where the constant $C_{9}$ does not depend on $y$.

Let $A=C_{9}\|f\|_{2}$, and $L_{v, \varepsilon}^{-1}$ be an operator inverse to $L_{v, \varepsilon}$. We denote $P_{\varepsilon}(v) \stackrel{\text { def }}{=} L_{v, \varepsilon}^{-1} f$. It follows from (8) that $P_{\varepsilon}(v)$ maps the ball $B_{A}$ into itself. Moreover $B_{A}$ is mapped to the set

$$
Q_{A} \stackrel{\text { def }}{=}\left\{y:\|y\|_{W} \leqslant C_{9}\|f\|_{2}\right\} .
$$

1. Since $Q_{A} \subset B_{A}$ then the set of the functions $Q_{A}$ is uniformly bounded

2. According to Morrey's inequality [18, p. 282] with $p=2$ for the functions $y \in W_{2}^{1}(\mathbb{R})$ the following inequality holds

$$
\|y\|_{C^{0, \frac{1}{2}(\mathbb{R})}} \leqslant C_{10}\|y\|_{W_{2}^{1}(\mathbb{R})}
$$


where $C^{0, \frac{1}{2}}(\mathbb{R})$ is a Hölder space with a norm

$$
\|y\|_{C^{0, \frac{1}{2}(\mathbb{R})}}=\sup _{\substack{a, b \in \mathbb{R} \\ a \neq b}} \frac{y(a)-y(b)}{\sqrt{|a-b|}} .
$$

Therefore, for any $y \in Q_{A}$

$$
|y(t+h)-y(t)| \leqslant C_{11} \sqrt{|h|},
$$

and hence functions from $Q_{A}$ are equicontinuous.

3. It follows from the estimate (8) that

$$
\sup _{x \in \mathbb{R}}\left|\left(1+x^{2}\right)^{\frac{\delta}{2}} y(x)\right| \leqslant A
$$

therefore, for any $y \in Q_{A}$ we have

$$
\sup _{x \in \mathbb{R}}|y(x)| \leqslant \frac{A}{\left(1+x^{2}\right)^{\frac{\delta}{2}}} \rightarrow 0 \text { as }|x| \rightarrow+\infty .
$$

Hence, the set $Q_{A}$ is compact in $C(\mathbb{R})$.

We consider a sequence of functions $\left\{v_{n}\right\}_{n=1}^{+\infty} \subset B_{A}$ such that $\left\|v-v_{n}\right\|_{C(\mathbb{R})} \rightarrow 0$ as $n \rightarrow+\infty$, and denote $P_{\varepsilon}\left(v_{n}\right)=y_{n}$. Then $L_{v_{n}, \varepsilon} y_{n}=f$ and by virtue of linearity of $L_{v, \varepsilon}$ we receive

$$
L_{v, \varepsilon}\left(y_{n}-y\right)=\left(r(x, v(x))-r\left(x, v_{n}(x)\right)\right) y_{n}^{\prime}+\left(s(x, v(x))-s\left(x, v_{n}(x)\right)\right) y_{n} .
$$

Therefore, for any $N>0$, taking into account that the functions $r(x, v(x))-r\left(x, v_{n}(x)\right)$ and $s(x, v(x))-$ $-s\left(x, v_{n}(x)\right)$ are continuous in $\mathbb{R}$, we get

$$
\begin{aligned}
\left\|y_{n}-y\right\|_{L_{2}(-N, N)} \leqslant C_{12} \max \left(\sup _{|x| \leqslant N}\left|r(x, v(x))-r\left(x, v_{n}(x)\right)\right|, \sup _{|x| \leqslant N}\left|s(x, v(x))-s\left(x, v_{n}(x)\right)\right|\right) \times & \\
& \times\left(\left\|y_{n}^{\prime}\right\|_{L_{2}(-N, N)}+\left\|y_{n}\right\|_{L_{2}(-N, N)}\right) \rightarrow 0,
\end{aligned}
$$

as $n \rightarrow+\infty$.

As $v_{n} \in B_{A}$ then $y_{n} \in Q_{A}$. Since $Q_{A}$ is a compact in $L_{2}$, and the operator $L_{v, \varepsilon}$ is closed then the Cauchy sequence $\left\{y_{n}\right\}_{n=1}^{+\infty}$ converges to the element $y \in Q_{A}$ (due to the uniqueness of the limit). Therefore $P_{\varepsilon}$ is a continuous operator.

Thus, the continuous operator $P_{\varepsilon}: B_{A} \rightarrow B_{A}$ maps the ball $B_{A}$ into itself, hence according to the Schauder theorem it has a fixed point, i. e. $\exists y \in B_{A}: P_{\varepsilon}(y)=y$. In other words $y$ satisfies the equation

$$
-\rho(x)\left(\rho(x) y^{\prime}\right)^{\prime}+\left(r(x, y)+\varepsilon\left(1+x^{2}\right)\right) y^{\prime}+s(x, y) y=f(x),
$$

by virtue of (8) the following estimate holds

$$
\left\|-\rho(x)\left(\rho(x) y^{\prime}\right)^{\prime}\right\|_{2}+\left\|\left(r(x, y)+\varepsilon\left(1+x^{2}\right)\right) y^{\prime}\right\|_{2}+\|s(x, y) y\|_{2} \leqslant C_{9}\|f\|_{2} .
$$

We consider a sequence of positive numbers $\left\{\varepsilon_{k}\right\}_{k=1}^{+\infty}$ tending to 0 . If $y_{k} \in B_{A}$ is a fixed point of the operator $P_{\varepsilon_{k}}$ then

and

$$
-\rho(x)\left(\rho(x) y_{k}^{\prime}\right)^{\prime}+\left(r\left(x, y_{k}\right)+\varepsilon_{k}\left(1+x^{2}\right)\right) y_{k}^{\prime}+s\left(x, y_{k}\right) y_{k}=f(x),
$$

$$
\left\|-\rho(x)\left(\rho(x) y_{k}^{\prime}\right)^{\prime}\right\|_{2}+\left\|\left(r\left(x, y_{k}\right)+\varepsilon_{k}\left(1+x^{2}\right)\right) y_{k}^{\prime}\right\|_{2}+\left\|s\left(x, y_{k}\right) y_{k}\right\|_{2} \leqslant C_{13}\left(\varepsilon_{k}\right)\|f\|_{2} .
$$

Let $[a, b] \subset \mathbb{R}$ be a finite segment. Since the space $W_{2}^{2}(a, b)$ is compactly embedded to $L_{2}(a, b)$ then there is a subsequence $\left\{y_{k_{i}}\right\}_{i=1}^{+\infty}$, converging to $y$ by the norm of $L_{2}(a, b)$, that is

$$
\lim _{i \rightarrow+\infty}\left\|y_{k_{i}}-y\right\|_{L_{2}(a, b)}=0 .
$$

Then according to the definition 1,y is the solution of the equation (1), and by virtue of (9) the following estimate holds

$$
\left\|-\rho(x)\left(\rho(x) y^{\prime}\right)^{\prime}\right\|_{2}+\left\|r(x, y) y^{\prime}\right\|_{2}+\|s(x, y) y\|_{2}<+\infty .
$$


The solvability conditions...

\section{Conclusion}

In this work we considered the conditions of the correct solvability as well as established a coercive estimate for the second-order differential equation (1) in a non-compact domain, and with coefficients that can be unbounded. In the case of a Hilbert space, this work generalizes the results of [17] to the nonlinear differential equation.

\section{Acknowledgments}

This research was funded by the Science Committee of the Ministry of Education and Science of the Republic of Kazakhstan (Grant No. AP08856281 "Nonlinear elliptic equations with unbounded coefficients").

\section{References}

1 Naimark, M.A. (1968). Linear Differential operators. London: George G.Harrap \& Co.

2 Reed, M., \& Simon, B. (1975). Methods of Modern Mathematical Physics: Fourier Analysis, Self-Adjointness. Academic Press.

3 Fedoruk, M.V. (1993). Asymptotic Analysis. Linear Ordinary Differential Equations. Berlin: Springer.

4 Bogachev, V.I., Krylov, N.V., Röckner, M., \& Shaposhnikov S.V. (2015). Fokker-Planck-Kolmogorov equations: Mathematical surveys and monographs. American Mathematical Society, Providence.

5 Fornaro, S., \& Lorenzi, L. (2007). Generation results for elliptic operators with unbounded diffusion coefficients in $L^{p}$-and $C_{b}$-spaces. Discrete and Continuous Dynamical Systems, 18(4), 747-772.

6 Hieber, M., \& Sawada, O. (2005). The Navier-Stokes Equations in $\mathbb{R}^{n}$ with Linearly Growing Initial Data. Archive for Rational Mechanics and Analysis, 175, 269-285.

7 Metafune, G., Pallara. D., \& Vespri, V. (2005). $L^{p}$-estimates for a class of elliptic operators with unbounded coefficients in $\mathbb{R}^{n}$. Houston Journal of Mathematics, 31, 605-620.

8 Hieber, M., Lorenzi, L., Prüss, J., Rhandi, A., \& Schnaubelt, R. (2009). Global properties of generalized Ornstein-Uhlenbeck operators on $L_{p}\left(\mathbb{R}^{N}, \mathbb{R}^{N}\right)$ with more than linearly growing coefficients. J. Math. Anal. Appl., 350, 100-121.

9 Ospanov, K.N,. \& Akhmetkaliyeva R.D. (2012). Separation and the existence theorem for second order nonlinear differential equation. Elec. J. Qual. Th. Dif. Eq., 66, 1-12.

10 Ospanov, K.N., \& Akhmetkaliyeva R.D. (2015). Some inequalities for secong order differential operators with unbounded drift. Eurasian Mathematical Journal, 6(2), 63-71.

11 Ospanov, K.N. (2015). $L_{1}$-maximal regularity for quasilinear second order differential equation with damped term. Elec. J. Qual. Th. Dif. Eq., 39, 1-9.

12 Ospanov, K.N. (2019). Maximal $L_{p}$-regularity for a second-order differential equation with unbounded intermediate coefficient. Elec. J. Qual. Th. Dif. Eq., 65, 1-13.

13 Muratbekov, M.B., Muratbekov, M.M., \& Abylayeva, A.M. (2013). On existence of the resolvent and discreteness of the spectrum of a class of differential operators of hyperbolic type. Elec. J. Qual. Th. Dif. Eq., $64,1-10$.

14 Muratbekov, M.B., \& Muratbekov, M.M. (2007). Estimates of the spectrum for a class of mixed type operators. Differential equations, 43(1), 143-146.

15 Zhapsarbayeva, L., \& Ospanov, K. (2020). Solvability of nonlinear problem for some second-order nonstrongly elliptic system. Complex Variables and Elliptic Equations, in appear.

16 Ospanov, K.N., Yeskabylova, Zh.B., \& Bekjan, T.N. (2019). The solvability results for the third-order singular non-linear differential equation. Eurasian mathematical journal, 10(4), 84-90.

17 Ospanov, K., \& Yesbayev, A. (2020). Solvability and maximal regularity results for a differential equation with diffusion coefficient. Turkish journal of mathematics, 44(4), 1304-1316.

18 Evans, L.C. (2010). Partial differential equations: Mathematical surveys and monographs. American Mathematical Society, Providence. 
А.Н. Есбаев, М.Н. Оспанов

\title{
$L_{2}(\mathbb{R})$-де шенелмеген коэффициенттері бар екінші ретті сызықтықемес дифференциалдық теңдеудің шешілу шарттары
}

\begin{abstract}
Мақалада шенелмеген облыста сызықтыемес екінші ретті дифференциалдық теңдеудің жалпы шешімінің бар болу мәселесі қарастырылған. Теңдеудің аралық және ең кіші коэффициенттері ізделген функцияға тәуелді және тегіс болып саналады. Жұмыстың жаңашылдығы - үлкен коэффициенті нөлден өзге болатын сызықтыемес сингулярлық теңдеудің шешілетіндігін дәлелдейтіндігімізде. Бұрын қарастырылғандардан айырмашылығы, теңдеудің үлкен коэффициенті нөлге ұмтылуы мүмкін, ал аралық коэффициент шексіздікке ұмтылады және ең кіші коэффициенттің өсуіне бағынбайды. Алынған нәтиже теңдеудің коэффициенттері бойынша тұжырымдалған; бұл коэффициенттердің кезкелген туындыларына шарттар қойылмайды.
\end{abstract}

Кілт сөздер: екінші ретті дифференциалдық теңдеу, сызықтыемес дифференциалдық теңдеу, шенелмеген облыстағы дифференциалдық теңдеу, жалпы шешім, шешімділік.

А.Н. Есбаев, М.Н. Оспанов

\section{Условия разрешимости нелинейного дифференциального уравнения второго порядка с неограниченными коэффициентами в $L_{2}(\mathbb{R})$}

В статье рассмотрен вопрос существования обобщённого решения нелинейного дифференциального уравнения второго порядка в неограниченной области. Промежуточный и младший коэффициенты уравнения зависят от искомой функции и считаются гладкими. Новизна работы состоит в том, что мы доказываем разрешимость нелинейного сингулярного уравнения с неотделённым от нуля старшим коэффициентом. В отличие от работ, рассмотренных ранее, старший коэффициент уравнения может стремиться к нулю, а промежуточный - к бесконечности и не подчиняться росту младшего коэффициента. Полученный результат сформулирован в терминах самих коэффициентов уравнения, в нём не ставятся условия на какие-либо производные этих коэффициентов.

Ключевые слова: дифференциальное уравнение второго порядка, нелинейное дифференциальное уравнение, дифференциальное уравнение в неограниченной области, обобщённое решение, разрешимость. 


\author{
A.R. Yeshkeyev ${ }^{*}$, N.M. Mussina \\ Buketov Karaganda University, Karaganda, Kazakhstan \\ (E-mail: aibat.kz@gmail.com,nazerke170493@mail.ru)
}

\title{
An algebra of the central types of the mutually model-consistent fragments
}

\begin{abstract}
In this paper, the model-theoretical properties of the algebra of central types of mutually model-consistent fragments are considered. Also, the connections between the center and the Jonsson theory in the permissible signature enrichment are shown, and within the framework of such enrichment, instead of some complete theory under consideration, we can obtain some complete 1-type, and we will call this type the central type, while the theories under consideration will be hereditary. Our work is divided into 3 sections: 1) the outer and inner worlds of the existentially closed model of the Jonsson theory (and the feature between these worlds is considered for two existentially closed models of this theory); 2) the $\lambda$-comparison of two existentially closed models (the Schroeder-Bernstein problem is adapted to the study of Jonsson theories in the form of a $J S B$-problem); 3) an algebra of central types (we carry over the results of Section 2 for the algebra $(\operatorname{Fr}(\bar{C}), \times)$, where $\bar{C}$ is the semantic model of the theory $\bar{T})$. Also in this article, the following new concepts have been introduced: the outer and inner worlds of one existentially closed model of the same theory (as well as the world of this model), a totally model-consistent Jonsson theory. The main result of our work shows that the properties of the algebra of Jonsson theories for the product of theories are used as an application to the central types of fixed enrichment. And it is easy to see from the definitions of the product of theories and hybrids that these concepts coincide if the product of two Jonsson theories gives a Jonsson theory.
\end{abstract}

Keywords: Jonsson theory, central types, $\varphi(x)$-set, outer world, inner world, $\lambda$-comparison, totally modelconsistent theory, fragment, algebra of the central types, semantical model.

In this article, we will consider an algebra that is related to the central types of some fixed Jonsson spectrum; more precisely, we work in one of the cosemanticness classes of some Jonsson spectrum. Moreover, the center of this class is a perfect, totally model-consistent theory. One of the special cases of the $J S B$-problem is also considered within the framework of the study of the class of existentially closed models of some fixed Jonsson theory. The problems related to the description of the syntactic and semantic properties of the Jonsson spectrum $J \operatorname{Sp}(A)$, where $A$ is an arbitrary model of an arbitrary signature, are new problems that appeared in the study of the cosemanticness properties of fixed Jonsson theories $[1 ; 80]$. The works related to this topic include the following works [2-5].

The central idea that led to this article is the idea of defining an operation between complete theories. As far as we know, the first source related to this idea that we were able to find is the work [6]. We would like to note that interest in this topic appeared after the reports of M.I. Bekenov [7] and A.M. Nurakunov [8] at various conferences, where they informed us about their achievements in the study of the algebra of theories concerning the operation introduced in $[6,9]$.

The concept of elementary equivalence between models of a complete theory is an important tool for comparing the similarity of these models, and one of the classical examples of applying this notion to algebra is the Keisler-Shelach theorem on the isomorphism of some ultrapower of elementarily equivalent algebras with each other [10; 363]. In [9] it was shown that elementary equivalence is preserved concerning the Cartesian product. Thus, the above operation, given on theories, preserves elementary equivalence.

The concept of cosemanticness $[11 ; 867]$ is a generalization of the concept of elementary equivalence. And this concept is directly related to the study of the model-theoretical properties of the Jonsson theory. As follows from the definition, Jonsson theories are, generally speaking, incomplete, so we cannot directly transfer the concept of an operation between Jonsson theories as a Cartesian product of their models, as announced in the

\footnotetext{
${ }^{*}$ Corresponding author.

E-mail: aibat.kz@gmail.com
} 
above reports [7], [8]. In particular, it should be noted that we are not working with the entire class of models of the fixed Jonsson theory, but only with the class of their existentially closed models. And there are 3 reasons for this: 1) as is well known from the definition of Jonsson's theory, any of its models is isomorphically embedded in some existentially closed model of this theory; 2) in the case of the perfectness of the considered Jonsson theory the class of its existentially closed models coincides with the class of all models of the center of considered Jonsson theory; 3) the semantic model of the considered Jonsson theory is an existentially closed model of this theory. Therefore, we will consider the concept of cosemanticness between the existentially closed models of this Jonsson theory.

The next aspect of the difference between our approach to the algebra of the considered Jonsson theories from the statement of the problem in the reports [7], [8] is the fact that we will deal with central types of a fixed Jonsson spectrum, that is, we will work in some enrichment of the original language.

The concept of a Jonsson spectrum was directly related to the concept of cosemanticness, both between models and theories. The definition of cosemanticness between models and between theories can be found in the following reference [12]. Some interest is the consideration of special definable subsets of the semantic model fixed Jonsson theory. In this regard, we draw the reader's attention to the following articles [13-15], which use various approaches in adapting the classical concepts of the model theory of arising in the study of complete theories to Jonsson theories.

In [6], the product of two theories was considered, and it was shown that this product preserves the stability properties in the product, if such are the factors.

It is well known that the concept of cosemanticness generalizes the concept of elementary equivalence, that is, if two models of some Jonsson theory of an arbitrary signature are elementarily equivalent to each other, then they are cosemantic to each other. Moreover, the notion of cosemanticness of two models is related to the notion of a Jonsson spectrum as follows: models $A \bowtie B$ if $J S p(A)=J S p(B)$.

Our task in this article is to adapt the above properties of the considered algebra of complete theories to study the model-theoretical properties of some fixed Jonsson theory.

Using the fact that the Jonsson theory $T$ is a special case of inductive theories, we note that the class $E_{T}$ is always not empty, and also that in inductive theory any model from the class ModT is isomorphically embedded into some model from the class $E_{T}$.

\section{The outer and inner worlds of the existentially closed model of the Jonsson theory}

The following definition defines the inner world $\left(I W_{T}(A)\right)$ of the model $A$ of the Jonsson theory $T$ when $A \in E_{T}$.

Definition 1. Let $T$ be an arbitrary Jonsson theory. $I W_{T}(A)=\left\{A^{\prime} \in E_{T} \mid f\right.$ is isomorphism, $f: A^{\prime} \rightarrow A$, $\left.A \in E_{T}\right\}$ is called the inner world of the model $A$ for $T$.

The following definition defines the outer world $\left(O W_{T}(A)\right)$ of the model $A$ of the Jonsson theory $T$ when $A \in E_{T}$.

Definition 2. Let $T$ be an arbitrary Jonsson theory. $O W_{T}(A)=\left\{B \in E_{T}\right.$ : there exist $\left.A^{\prime} \cong A, A^{\prime} \subseteq B\right\}$ is called the outer world of the model $A$ for $T$.

And just the world of the existentially closed model $A$ will be the following set

$$
W_{T}(A)=I W_{T}(A) \cup O W_{T}(A) .
$$

Note that the above definitions can connect two different existentially closed models in the case of a convex theory. As the following theorem is true.

Theorem 1. Let $T$ be the perfect, strong convex Jonsson theory. Since for any models $A, B \in E_{T}$ the following is true:

1) $O W_{T}(A) \cap O W_{T}(B) \neq \emptyset$

2) $I W_{T}(A) \cap I W_{T}(B) \neq \emptyset$.

Proof. By virtue of the perfectness of the theory $T, E_{T}=\operatorname{Mod} T^{*}$. And all existentially closed models of the theory $T$ are models of the center of the theory $T$, therefore property 1 ) is true due to the fact that models $A$ and $B$ are existentially closed submodels of the semantic model $C$, where $C$ - semantic model of the theory $T$. Due to the strongly convexity of the theory $T$, the intersection of any two models is not empty. Condition 2) is trivial and is performed due to the joint embedding property $(J E P)$ of the theory $T$. In particular, the model $C$ satisfies these conditions due to the $T^{+}$universality of the model $C$. 


\section{The $\lambda$-comparison of two existentially closed models}

In model theory, the formulation of the following problem is well known, which is called the SchroderBernstein problem $(S B)$ [16]. This question concerns the isomorphism of two structures, which are mutually elementary embedded in each other. This topic was adapted to the study of Jonsson theories in the form of a $J S B$-problem. In particular, one can refer to [11], where the $J S B$-problem is studied in the framework of Jonsson Abelian groups.

In this section, we will consider a special case of this problem, namely, the $\lambda$-comparison of two existentially closed models of Jonsson theory.

Definition 3. Let $T$ be a Jonsson theory. Let $\omega \leq \lambda \leq \mu, A$ and $B$ be existentially closed models of the theory T. $|A|=|B|=|\mu|$. Models $A$ and $B$ will be called the $\lambda$-comparable if for any existentially closed submodel $A^{\prime}$ of a model $A$, such that $\left|A^{\prime}\right| \leq \lambda$, it is true that $A^{\prime}$ is an existentially closed submodel of $B$, and for any existentially closed submodel $B^{\prime}$ of a model $B$, such that $\left|B^{\prime}\right| \leq \lambda$, it is true that $B^{\prime}$ is an existentially closed submodel of $A$.

It is clear that the above definition defines an equivalence relation on the set of all existentially closed models of the considered Jonsson theory. Therefore, the following spectral definition of the number of model classes makes sense.

Definition 4. Let $T$ be a Jonsson theory, $\omega \leq \lambda \leq \mu . N\left(E_{T}^{\lambda, \mu}\right)$ calculates the number of classes of existentially closed models of Jonsson theory of cardinality $\mu$ concerning the $\lambda$-comparison relation.

Definition 5. Let $I W_{T}^{\lambda}(A)$ be the set of all models from $I W_{T}(A)$, whose cardinality does not exceed $\lambda$.

Theorem 2. Let $T$ be $\exists$-complete, Jonsson theory, and for some $\omega \leq \lambda \leq \mu$ holds $N\left(E_{T}^{\lambda, \mu}\right)=1$. Then the theory $T^{*}$ is model complete.

Proof. Let there exist $\omega \leq \lambda \leq \mu$, such that $N\left(E_{T}^{\lambda, \mu}\right)=1$. It means that for any two models $A, B$, $|A|=|B|=|\mu|, A, B \in E_{T}, I W_{T}^{\lambda}(A)=I W_{T}^{\lambda}(B)$. Using the fact that all models from $I W_{T}^{\lambda}(A)$ are isomorphic to each other due to the condition $N\left(E_{T}^{\lambda, \mu}\right)=1$, we fix an isomorphism between any two models $M_{1}, M_{2}$ from $I W_{T}^{\lambda}(A)$ and $I W_{T}^{\lambda}(B)$. Further, in view of the $\exists$-completeness of the theory $T$ and the $k^{+}$-homogeneity of the semantic model $C$ of the theory $T$, we can extend this isomorphism to an automorphism $C$.It follows that the outer worlds are $O W_{T}\left(M_{1}\right) \uparrow A=O W_{T}\left(M_{2}\right) \uparrow B$. Since $W_{T}\left(M_{1}\right)=W_{T}\left(M_{2}\right)$, we have that $A \cong B$. Those in cardinality $\mu$ he theory $T$ in the class $E_{T}$ has only one model up to isomorphism, the theory $T$ is $\mu$-categorical, which means that it is $T$-perfect. In this case, that $T^{*}$ is a model companion of the theory $T$, and respectively, $T^{*}$ is model complete.

Let us give the necessary definitions of concepts from the above sources and list the results announced in the reports [7], [8].

In what follows, let $T$ be some fixed perfect Jonsson theory, $C$ is a semantic model of the theory $T$.

The product of two complete theories in an arbitrary first-order language $L$ is defined as follows.

Let us give the definition of a product of fixed mutually model-consistent Jonsson theories in a countable language of an arbitrary signature $\sigma$.

Definition 6 . Let $T_{1}, T_{2}$ be Jonsson theories and $T_{1}, T_{2}$ mutually model-consistent.Then we define $T_{1} \times T_{2}$ as the following theory: $T h_{\forall \exists}\left(C_{1} \times C_{2}\right)$, where $C_{1}, C_{2}$ are semantic models of Jonsson theories $T_{1}, T_{2}$, respectively in cardinality $2^{k}$, where $k \geq \omega$.

In particular, if we consider Jonsson's horn theory, then since it is not necessarily complete, we can isolate all of its completion. And as an example of an operation for complete theories, we can consider an operation between completions on the set of all completions.

Due to the fact that the semantic model of some Jonsson theory, which specifies the cosemanticness of these two theories, is existentially closed, it does not yet follow that the Cartesian product of two existentially closed submodels of the semantic model will be an existentially closed submodel of this semantic model.

If $X$ is an arbitrary definable subset of the semantic model $C$ of the Jonsson theory $T$ and its closure $\operatorname{cl}(X)=M$ in some pregeometry given on the Boolean $C$, then the following Jonsson theory is $T h_{\forall \exists}(M)$, denoted by $\operatorname{Fr}(X)$, we will call a fragment in the theory $T$. It is easy to see that if $M \in E_{T}$, then $\operatorname{Fr}(X)$ is always Jonsson theory. In this article, we do not consider the content of the set $X$.

Let $\operatorname{Fr}(C)$ denote the set of all fragments in the theory $T$.

Definition \%. A Jonsson theory $T$ is called totally model-consistent if any of its two fragments $\operatorname{Fr}\left(X_{1}\right)$, $\operatorname{Fr}\left(X_{2}\right) \in \operatorname{Fr}(C)$ are mutually model-consistent, where $X_{1}, X_{2}$ are some definable subsets of $C$.

It seems to us a great prospect is filled with real meaning that the concept of definability of a subset of the semantic model through the concept of $\varphi(x)$-set. For example, when $\varphi(x)$ expresses: $A P A$-transcendence, 
various kinds of atomicity and primeness, strongly minimality, Jonssonness. And finally, the description of the closure of the $\varphi(x)$-set will be obtained through the $\varphi(x)$-rheostat [17].

In what follows, we will work within the framework of a fixed totally model-consistent Jonsson theory. It is clear that in this case, the question arises: is the cosemanticness class of Jonsson theories perfect if its center is model-consistent? That is, the question arises: in which case of the center will the following theorem be true?

Theorem 3. If the fixed center of some cosemanticness class of the Jonsson spectrum is totally modelconsistent, then its semantic model is saturated.

The essence of the question lies in filling with the meaning of the word fixed.

Definition 8. For each fragment $\nabla$ from the Jonsson theory $T$ select the following set $N \nabla=\{\Delta \mid \Delta \in$ $\in \operatorname{Fr}(C), \Delta \times \nabla=\nabla\}$. Let $D \subseteq \operatorname{Fr}(C)$. If $D=N \nabla$ for some $\nabla \in \operatorname{Fr}(C)$, then $D$ is called a definable set of the theory $\nabla$.

Let $\Delta_{1}, \Delta_{2}, \ldots \Delta_{n}, \Delta \in \operatorname{Fr}(C)$.

Consequence 1. For any $\Delta \in \operatorname{Fr}(C), N \Delta$ is non-empty and closed with respect to finite products.

Proof. $E$ is the theory of one-element model, then $E \times \Delta=\Delta$. Hence, $N \Delta$ is not empty. Let $\Delta_{1}, \ldots, \Delta_{n}$ be from $N \Delta$, then $\Delta_{1} \times \ldots \times \Delta_{n} \times \Delta=\Delta$, that is, closed with respect to finite products.

Remark 1. Generally speaking, the theory $\Delta$ can not belong to $N \Delta$, and $N \Delta$ can not be closed with respect to infinite products, and there are various $\nabla_{1}, \nabla_{2}$, such that $N \nabla_{1}=N \nabla_{2}$, that is, they have the same definable set $N$.

Definition 9. If $\Delta_{1} \times \Delta_{2}=\Delta_{2}$, then we will write $\Delta_{2}$ absorbs $\Delta_{1} . \Delta$ is called an idempotent if $\Delta \times \Delta=\Delta$.

Definition 10. A set $D \subseteq \operatorname{Fr}(C)$ is called a definable idempotent if there exists an idempotent $\Delta$, such that $N \Delta=D$.

Jonsson's version of Weinstein's theorem [10; 416].

Theorem 4. Let $A, B, C \in E_{T}$. If $A \bowtie(A \times B \times C)$, then $A \bowtie(A \times B)$ ( $\bowtie$ is cosmanticness of models).

Proof. The elementary equivalence of the two models implies that they are cosemantic.

Theorem 5. If $\Delta_{1}=\Delta_{1} \times \Delta_{2} \times \Delta_{3}$, then $\Delta_{1}=\Delta_{1} \times \Delta_{2}$.

Proof. Follows from Theorem 5 and Theorem 4.

Theorem 6 (Vaught [10; 403]). A sentence in the language $L$ is stable with respect to infinite direct products of algebraic systems if it is stable with respect to any finite subproducts of this infinite product.

Theorem \%. If $D \subseteq \operatorname{Fr}(C)$, and $D$ is closed under infinite products, then there is a unique idempotent $\Delta \in D$, that absorbs any element from $D$.

For example, the set of all theories that are complete extensions of the theory of some quasivariety are absorbed by the corresponding idempotent, but this idempotent may not define this set.

Theorem 8. Let $D \subseteq \operatorname{Fr}(C)$, and $D$ is a definable set closed with respect to infinite products, then there is a unique idempotent $\Delta \in D$ such that $D$ is definable by this idempotent.

Proof. Let $D=N \Delta$ for some theory $\Delta$. By Theorem 7 , there is an idempotent $\Delta_{2}$, which absorbs all elements from $D$. Idempotent $\Delta_{2}$ defines the set $D$. Indeed, if for some theory $\Delta_{1}$ holds $\Delta_{2} \times \Delta_{1}=\Delta_{2}$, then $\Delta \times \Delta_{2} \times \Delta_{1}=\Delta$. This means that $\Delta \times \Delta_{1}=\Delta$. That is, $\Delta_{1} \in D$.

Theorem 9. If $\Delta$ is idempotent, then its any power is $\Delta^{k}=\Delta$.

Proof. The proof follows from the above definitions.

Theorem 10. If $\Delta$ is idempotent, then $N \Delta$ is closed with respect to the products.

Proof. Let $N \subseteq N \Delta$ and $N=\left\{\Delta_{i} \mid i \in I\right\}$. Take the product of all $\Delta_{i} \in N$. We denote this product by $P$. Since $\Delta$ is idempotent, we can write $\Delta=\Delta \times \Delta \times \ldots=\left(\Delta_{1} \times \Delta\right) \times\left(\Delta_{2} \times \Delta\right) \times \ldots=\Delta_{1} \times \Delta_{2} \times \ldots \times \Delta \times \Delta=P \times \Delta$. То есть $P \in N \Delta$.

If we use the well-known theorem: „For any families $\left\{A_{i} \mid i \in I\right\},\left\{B_{i} \mid i \in I\right\}$ of algebraic systems and any filter $D$ over $I$, holds $\prod_{i \in I}\left(A_{i} \times B_{i}\right) / D=\prod_{i \in I} A_{i} / D \times \prod_{i \in I} B_{i} / D$ ". The following is true.

All the remaining statements in this section (Theorem 11-17, Corollary 2-3) are Jonsson analogs of the corresponding results from the unpublished paper [18].

Theorem 11. If $\Delta$ is idempotent, then $N \Delta$ is an axiomatizable theory.

Theorem 12. Let $I d=\{\Delta \mid \Delta \in \operatorname{Fr}(C), \Delta$-idempotent $\}$. Id with the product operation forms a commutative semigroup of idempotents with identity.

Corollary 2. The semigroup of idempotents $H$ is isomorphically embeddable into a suitable power of the semigroup $J$, where $J$ is the semigroup with base set $\{0,1\}$ and the corresponding operation.

Thus, it is possible to represent idempotents as sequences of zeros and ones.

Definition 11. We will write $\Delta_{1} \leq \Delta_{2}$, if and only if $\Delta_{1} \times \Delta_{2}=\Delta_{2}$.

Corollary 3. The relation $\leq$ on the set $\operatorname{Fr}(C)$ is a partial order with the largest and the smallest elements.

Theorem 13. Let $\Delta_{1}, \Delta_{2} \in I d$. The set $N \nabla_{1} \cap N \nabla_{2}$ is definable by an idempotent. 
Theorem 14. Let $\Delta_{1}, \Delta_{2}, \Delta_{3} \in I d$ and $\Delta_{1} \leq \Delta_{3}, \Delta_{2} \leq \Delta_{3}$. Then $\Delta_{1} \times \Delta_{2} \leq \Delta_{3}$.

Definition 12. On the set $I d$ we introduce the operations of union and intersection. Let $\Delta_{1}, \Delta_{2} \in I d$. $\Delta_{1} \cup \Delta_{2}=\Delta_{1} \times \Delta_{2}$, and $\Delta_{1} \cap \Delta_{2}=\Delta$, where $\Delta$ is from the proof of Theorem 13 .

Theorem 15. The set $I d$ with operations $\cup$ and $\cap$ forms a complete lattice.

Theorem 16. Each variety is associated with its unique defining idempotent.

Theorem 1\%. The set of definable quasivarieties with respect to the introduced operations $\cup$ and $\cap$ form a complete lattice.

\section{An algebra of the central types}

Let us give the necessary definitions related to a special kind of enrichment of the Jonsson theory, which preserves certain properties. Within the framework of such enrichment, as a consequence of the laws of the logic of the predicate calculus, instead of the considered some complete theory, we can obtain a maximally consistent set of formulas, i.e. some complete 1-type. We will call this type the central type.

The main idea of this section is to use, as an application to central types of fixed enrichment, the properties of the algebra of Jonsson theories with respect to the products of theories.

Let $T$ be a fixed hereditary Jonsson theory in the language $L$ of some signature $\sigma, C$ is a semantic model of this theory, $\sigma^{\prime}=\sigma \bigcup\{P\} \bigcup\{c\}$, take the set $A$, as a subset of $C$.

Let $\bar{T}=T \bigcup T h_{\forall \exists}\left(C, c_{a}\right)_{a \in A} \bigcup\{P(c)\} \bigcup\{P, \subseteq\}$. Here $\{P, \subseteq\}$ is an infinite set of sentences that reflect the following fact: „An interpretation of the symbol $P$ will be an existentially closed submodel on $L$ of signature $\sigma^{\prime \prime \prime}$, thus, we obtain that the interpretation of the symbol $P$ is a solution of the equation $P(C)=M, M \in E_{T}$ in $L$ of signature $\sigma^{\prime}$.

Due to the fact that not all Jonsson theories in enrichment preserve the property of being a Jonsson theory, we will work in the class of hereditary Jonsson theories. "What is it?" - give the following definitions.

Definition 13. An enrichment $\bar{T}$ of the Jonsson theory $T$ is said to be permissible if any $\nabla$-type (it mean that $\nabla$ subset of language $L_{\sigma}$ and any formula from this type belongs to $\nabla$ ) in this enrichment is definable in the framework of $\bar{T}_{\Gamma^{-} \text {-stability. }}$

Definition 14. The Jonsson theory is said to be hereditary, if in any of its permissible enrichment, any expansion of it in this enrichment will be Jonsson theory.

Let $T$ be a fixed hereditary Jonsson theory that is totally model-consistent, $C$ its semantic model, $\operatorname{Fr}(C)$ the set of all fragments in the theory $T$. On the set $\operatorname{Fr}(C)$ we define the operation $(\times)$, which will be the algebra $(\operatorname{Fr}(C), \times)$. And it forms a commutative semigroup of idempotents with unity, and the set of idempotents with operations $\cup$ and $\cap$ forms a complete lattice. Also applicable to the algebra $(\operatorname{Fr}(\bar{C}), \times)$, where $\bar{C}$ is the semantic model of the theory $\bar{T}$. And we have the following results when considering this algebra.

The operation of multiplying fragments from $\operatorname{Fr}(C)$ induces the operation of multiplication in $\operatorname{Fr}(\bar{C})$, and we can notice that $\operatorname{Fr}(\bar{C})$, as well as $\operatorname{Fr}(C)$, is a commutative semigroup for multiplication of fragments in the theory $\bar{T}$.

Since the considered theory $T$ is totally model-consistent, the product of two fragments from $\operatorname{Fr}(C)$ is a theory from $\operatorname{Fr}(C)$, that is, $\operatorname{Fr}(C)$ is the cosemantic class of the Jonsson spectrum of some model of the signature of the theory $T$.

It is clear that this topic, namely, the algebraization of the field of activity of work with central types, is closely to the questions arising in the study of Jonsson's theories. Let's dwell on one of them. This question concerns hybrids of Jonsson theories [19-21].

It is easy to see from the definitions of the product of theories and hybrids that these concepts coincide if the product of two Jonsson theories gives a Jonsson theory. In this case, the product of the central types of Jonsson theories differs from the concept of a hybrid in the following way: 1) to define the central type, some enrichment of the signature is necessary, difference to hybrids of Jonsson theories; 2) a hybrid of Jonsson's theories can be of 2 types (the first and second types), while the second type of hybrid depends on the Jonsson theories of two different signatures, difference to the product of Jonsson theories.

\section{Acknowledgments}

This work was supported by the Science Committee of the Ministry of Education and Science of the Republic of Kazakhstan (grant AP09260237). 


\section{References}

1 Barwise J. Ed., Handbook of mathematical logic. Part 1, Model Theory / J. Barwise. - M.: Science, 1982.

2 Yeshkeyev A.R. Properties of lattices of the existential formulas of Jonsson fragments / A.R. Yeshkeyev, M.T. Kasymetova // Bulletin of the Karaganda University. Mathematics series. - 2015. - 79. - № 3. P. 25-32.

3 Poizat B. Positive Jonsson Theories / B. Poizat, A.R. Yeshkeyev // Logica Universalis. — 2018. - 12. № 1-2. - P. 101-127.

4 Yeshkeyev A.R. del-cl-atomic and prime sets / A.R. Yeshkeyev, A.K. Issayeva // Bulletin of the Karaganda University. Mathematics series. - 2019. - 93. - № 1. - P. 88-94. DOI: 10.31489/2019M1/88-94

5 Yeshkeyev A.R. The Properties of Similarity for Jonsson's Theories and Their Models / A.R. Yeshkeyev // Bulletin of the Karaganda University. Mathematics series. - 2015. - 80. — № 4. - P. 52-59.

6 Wierzejewski J. On stability and products / J. Wierzejewski // Fundamenta Mathematicae. - 1976. 93. - P. 81-95.

7 Bekenov M.I. Semigroup of theories and lattices of idempotent elements / M.I. Bekenov // Program and Abstracts of The 16th Asian Logic Conference, Nur-Sultan. - 2019. - P. 36-37.

8 Нуракунов А.М. Полугруппа теорий и ее решетка идемпотентных теорий / А.М. Нуракунов, М.И. Бекенов // Мальцевские чтения-2020. - Новосибирск, 2020.

9 Weinstein J.M. First order properties preserved by direct product / J.M. Weinstein // Ph.D. thesis. Univ. Wisconsin, Madison, Wis. - 1965.

10 Кейслер Х.Дж. Теория моделей / Х.Дж. Кейслер, Ч.Ч. Чэн. - М.: Мир, 1977. - 614 с.

11 Ешкеев А.Р. $J S p$-косемантичность и $J S B$-свойство абелевых групп [Электронный ресурс] А.Р. Ешкеев, О.И. Ульбрихт // Siberian Electronic Mathematical Reports. - 2016. - 13. - C. 861-874. Режим доступа: http://semr.math.nsc.r Doi: 10.17377/semi.2016.13.068

12 Ешкеев А.Р. Йонсоновские теории и их классы моделей: моногр. / А.Р. Ешкеев, М.Т. Касыметова. - Караганда: Изд-во КарГУ, 2016. - 370 с.

13 Yeshkeyev A.R. The atomic definable subsets of semantic model / A.R. Yeshkeyev, N.M. Mussina, A.K. Issayeva // Bulletin of the Karaganda University. Mathematics series. - 2019. — 94. - № 2. P. 84-91. DOI 10.31489/2019M2/84-91

14 Yeshkeyev A.R. Companions of the fragments in the Jonsson enrichment / A.R. Yeshkeyev // Bulletin of the Karaganda University. Mathematics series. — 2017. — 85. — № 1. - P. 41-45. DOI: 10.31489/2017M1/4145

15 Ешкеев A.P. JSp-косемантичность $R$-модулей [Электронный ресурс] / A.P. Ешкеев, О.И. Ульбрихт // Siberian electronics mathematical reports. - 2019. - 16. - С. 1233-1244. - Режим доступа: http://semr.math.nsc.ru DOI: 10.33048/semi.2019.16.084

16 Goodrick J. The Schröder-Bernstein property for a-saturated models / J. Goodrick, M.C. Laskowski // Proc. AMS - 2014. - 142. - 3. - P. 1013-1023. Zbl 06268279

17 Yeshkeyev A.R. Method of the rheostat for studying properties of fragments of theoretical sets A.R. Yeshkeyev // Bulletin of the Karaganda University. Mathematics series. - 2020. - 100. - № 4. P. 152-159. DOI 10.31489/2020M4/152-159

18 Bekenov M.I. Semigroup of theories and its lattice of idempotent elements / M.I. Bekenov, A.M. Nurakunov // Algebra and Logic. - 2021.

19 Yeshkeyev A.R. Properties of hybrids of Jonsson theories / A.R. Yeshkeyev, N.M. Mussina // Bulletin of the Karaganda University. Mathematics series. - 2018. - 92. - № 4. - P. 99-104. DOI: 10.31489 /2018M4/99-104

20 Yeshkeyev A.R. Small models of hybrids for special subclasses of Jonsson theories / A.R. Yeshkeyev, N.M. Mussina // Bulletin of the Karaganda University. Mathematics series. - 2019. - 95. - № 3. P. 68-73.

21 Yeshkeyev A.R. Chains of existentially closed models of positive (n(1), n(2))-Jonsson theories / A.R. Yeshkeyev, M.T. Omarova // Bulletin of the Karaganda University. Mathematics series. - 2019. 96. 一 № 4. - P. 69-74. DOI 10.31489/2019M4/69-74 
А.Р. Ешкеев, Н.М. Мусина

\title{
Өзара модельді-үйлесімді фрагменттерінің централдық типтерінің алгебрасы
}

\begin{abstract}
Мақалада өзара модельді-үйлесімді фрагменттердің централдық типтерінің алгебрасының модельді-теоретикалық қасиеттері қарастырылған. Централдық және йонсондық теорияның байытылған сигнатурасының байланыстары да көрсетілген және осындай байыту аясында қарастырылған кейбір толық теорияның орнына авторлар толық 1-типті алған және бұл типті централдық тип деп атап, ал теориялардың орнына мұралы теорияларды қарастырған. Бұл жұмыс 3 бөлімге бөлінген: 1) йонсондық теорияның экзистенциалды тұйық моделінің сыртқы және ішкі әлемдері (берілген теорияның екі экзистенциалды тұйық модельдердің әлемдерінің арасындағы ерекшелік қарастырылған); 2) екі экзистенциалды тұйық модельдерді $\lambda$-салыстыру (Шредер-Бернштейн проблемасы йонсондық теорияларды $J S B$-проблемасы түрінде зерттеуге бейімделген); 3) централдық типтердің алгебрасы (2-бөлімнің нәтижелері $(\operatorname{Fr}(\bar{C}), \times)$ алгебрасына көшірілген, мұндағы $\bar{C}-\bar{T}$ теориясының семантикалық моделі). Бұдан басқа мақалада мына тұжырымдамалар енгізілген: теорияның экзистенциалды тұйық моделінің сыртқы және ішкі әлемі (сонымен қатар осы модельдің әлемі), тұтас модельді үйлесімді йонсондық теория. Жұмыстың негізгі нәтижесі көрсеткендей, теориялардың көбейтіндісіне қатысты йонсондық теориялардың алгебрасының қасиеттері бекітілген, байытылған централдық типтеріне қосымша ретінде қолданылады. Теориялардың көбейтіндісі мен гибридтердің анықтамаларынан, егер екі йонсондық теорияның көбейтіндісі йонсондық теорияны берсе, бұл ұғымдардың сәйкес келетіндігін байқау қиын емес.
\end{abstract}

Kiлm сөздер: йонсондық теория, централдық типтер, $\varphi(x)$-жиын, ішкі әлем, сыртқы әлем, $\lambda$-салыстыру, тұтас модельді үйлесімді теория, фрагмент, централдық типтердің алгебрасы, семантикалық модель.

\author{
А.Р. Ешкеев, Н.М. Мусина
}

\section{Алгебра центральных типов взаимно модельно-совместных фрагментов}

\begin{abstract}
В статье рассмотрены теоретико-модельные свойства алгебры центральных типов взаимно модельносовместных фрагментов. Показаны связи между центром и йонсоновской теорией в разрешенном обогащении сигнатуры. В рамках такого обогащения вместо рассматриваемой некоторой полной теории авторы могут получить некоторый полный 1 тип, и он будет называться центральным, при этом рассматриваемые теории будут являться наследственными. Данная работа разбита на 3 параграфа: 1) внешний и внутренний миры экзистенциально замкнутой модели йонсоновской теории (рассмотрена особенность между этими мирами для двух экзистенциально замкнутых моделей данной теории); 2) $\lambda$-сравнение двух экзистенциально замкнутых моделей (проблема Шредера-Бернштэйна адаптирована к изучению йонсоновских теорий в виде $J S B$-проблемы); 3$)$ алгебра центральных типов (результаты параграфа 2 переносятся для алгебры $(\operatorname{Fr}(\bar{C}), \times)$, где $\bar{C}$ - это семантическая модель теории $\bar{T})$. Кроме того, авторами введены следующие понятия: внешний и внутренний миры одной экзистенциально замкнутой модели одной и той же теории (а также мир этой модели); тотально модельно-совместная йонсоновская теория. Главный результат, достигнутый в работе, показывает, что в качестве приложения к центральным типам фиксированного обогащения используются свойства алгебры йонсоновских теорий относительно произведения теорий. И легко заметить из определений произведения теорий и гибридов, что эти понятия совпадают, если произведение двух йонсоновских теорий дает йонсоновскую теорию.
\end{abstract}

Ключевые слова: йонсоновская теория, центральные типы, $\varphi(x)$-множество, внешний мир, внутренний мир, $\lambda$-сравнение, тотально модельно-совместная теория, фрагмент, алгебра центральных типов, семантическая модель. 


\section{References}

1 Barwise, J. (1982). Ed. Handbook of mathematical logic. Part 1, Model Theory. Moscow, Science.

2 Yeshkeyev, A.R., \& Kasymetova, M.T. (2015). Properties of lattices of the existential formulas of Jonsson fragments. Bulletin of the Karaganda University - Mathematics, 79(3), 25-32.

3 Poizat, B., \& Yeshkeyev, A. R. (2018). Positive Jonsson Theories. Logica Universalis, 12(1-2), 101-127.

4 Yeshkeyev, A.R., \& Issayeva, A.K. (2019). del-cl-atomic and prime sets. Bulletin of the Karaganda University - Mathematics, 93(1), 88-94. DOI: 10.31489/2019M1/88-94

5 Yeshkeyev, A.R. (2015). The Properties of Similarity for Jonsson's Theories and Their Models. Bulletin of the Karaganda University - Mathematics, 80(4), 52-59.

6 Wierzejewski, J. (1976). On stability and products. Fundamenta Mathematicae, 93, 81-95.

7 Bekenov, M.I. (2019). Semigroup of theories and lattices of idempotent elements. Program and Abstracts of The 16th Asian Logic Conference, 36-37.

8 Nurakunov, A.M., \& Bekenov, M.I. (2020). Poluhruppa teorii i ee reshetka idempotentnykh teorii [Semigroup of theories and its lattice of idempotent theories]. Maltsevskiie chteniia-2020 - Maltsev Readings2020 [in Russian].

9 Weinstein, J.M. (1965). First order properties preserved by direct product. Ph.D. thesis. Univ. Wisconsin. Madison, Wis.

10 Keisler, H.J., \& Chang, C.C. (1977). Teoriia modelei [Model Theory]. Moscow: Mir [in Russian].

11 Yeshkeyev, A.R., \& Ulbrikht, O.I. (2016). JSp-kosemantichnost i $J S B$-svoistvo abelevykh hrupp [JSp-cosemanticness and JSB property of Abelian groups]. Sibirskie elektronnye matematicheskie izvestiia - Siberian Electronic Mathematical Reports, 13, 861-874 [in Russian].

12 Yeshkeyev, A.R., \& Kassymetova, M.T. (2016). Yonsonovskie teorii i ikh klassy modelei /Jonsson theories and their classes of models]. Karaganda: Izdatelstvo Karahandinskoho universiteta [in Russian].

13 Yeshkeyev A.R., Mussina, N.M., \& Issayeva, A.K. (2019). The atomic definable subsets of semantic model. Bulletin of the Karaganda University - Mathematics, 94(2), 84-91. Doi: 10.31489/2019M2/84-91

14 Yeshkeyev, A.R. (2017). Companions of the fragments in the Jonsson enrichment. Bulletin of the Karaganda University - Mathematics, 85(1), 41-45. Doi: 10.31489/2017M1/41-45

15 Yeshkeyev, A.R., \& Ulbrikht, O.I. (2019). JSp-kosemantichnost $R$-modulei [JSp-cosemanticness of R-modules]. Sibirskie elektronnye matematicheskie izvestiia - Siberian Electronic Mathematical Reports, 16, 1233-1244. DOI: 10.33048/semi.2019.16.084 [in Russian].

16 Goodrick, J., \& Laskowski, M.C. (2014). The Schroder-Bernstein property for a-saturated models Proc. AMS, 142(3), 1013-1023. Zbl 06268279

17 Yeshkeyev, A.R. (2020). Method of the rheostat for studying properties of fragments of theoretical sets. Bulletin of the Karaganda University - Mathematics, 100(4), 152-159. DOI 10.31489/2020M4/152-159

18 Bekenov, M.I., \& Nurakunov, A.M. (2021). Semigroup of theories and its lattice of idempotent elements. Algebra and Logic.

19 Yeshkeyev, A.R., \& Musina, N.M. (2018). Properties of hybrids of Jonsson theories. Bulletin of the Karaganda University - Mathematics, 92(4), 99-104. DOI: 10.31489/2018M4/99-104

20 Yeshkeyev, A.R., \& Mussina, N.M. (2019). Small models of hybrids for special subclasses of Jonsson theories. Bulletin of the Karaganda University - Mathematics, 95(3), 68-73.

21 Yeshkeyev, A.R., \& Omarova, M.T. (2019). Chains of existentially closed models of positive (n(1), n(2))-Jonsson theories. Bulletin of the Karaganda University - Mathematics, 96(4), 69-74. 
An essential base of the central types...

DOI $10.31489 / 2021 \mathrm{M} 1 / 119-126$

UDC 510.67

\author{
A.R. Yeshkeyev*, M.T. Omarova \\ Buketov Karaganda University, Karaganda, Kazakhstan \\ (E-mail: aibat.kz@gmail.com,omarovamt_963@mail.ru)
}

\title{
An essential base of the central types of the convex theory
}

\begin{abstract}
In this paper, we consider the model-theoretical properties of the essential base of the central types of convex theory. Also shows the connection between the center and Jonsson theory in permissible enrichment signatures. Moreover, the theories under consideration are hereditary. This article is divided into 2 sections: 1) an essential types and an essential base of central types (in this case, the concepts of an essential type and an essential base are defined using the Rudin-Keisler order on the set of central types of some hereditary Jonsson theory in the permissible enrichment); 2) the atomicity and the primeness of $\varphi(x)$-sets. In this paper, new concepts are introduced: the $\varphi(x)$-Jonsson set, the $A P A$-set, the $A P A$-existentially closed model, the $\varphi(x)$-convex theory, the $\varphi(x)$-transcendental theory, the $A P A$-transcendental theory. One of the ideas of this article refers to the fact that in the work of Mustafin T.G. it was noticed that any universal model of a quasi-transcendental theory with a strong base is saturated, but we generalized this result taking into account that: the concept of quasi-transcendence will be replaced by the $\varphi(x)$-transcendence, where $\varphi(x)$ defines some Jonsson set; and the notion of a strong base is replaced by the notion of an essential base, but in a permissible enrichment of the hereditary Jonsson theory. The main result of our work shows that the number of fragments obtained under a closure of an algebraic or definable type does not exceed the number of homogeneous models of a some Jonsson theory, which is obtained as a result of a permissible enrichment of the hereditary Jonsson theory.
\end{abstract}

Keywords: Jonsson theory, central types, essential base, $\varphi(x)$-set, $\varphi(x)$-convex theory, $\varphi(x)$-transcendental theory, APA-transcendental theory, fragment.

This article is devoted to the study of the central types of a special case of the convex theory [1]. Besides, the concept of convexity is considered class of Jonsson theories [2; 80]. Many works were devoted to the study of Jonsson theories: the general properties of Jonsson theories, for example, were studied in the following papers [3-7]; results concerning various extensions of Jonsson theories, including companions, can be found in [8, 9]. When studying the properties of elements and subsets of the semantic model of the fixed Jonsson theory, many new concepts and problems related to them have arisen. First of all, it should be noted the concept of a Jonsson set, which is a generalization of the concept of a basis in a linear space. If we consider a linear space as a special case of a module, then our interest in studying various refinements of the concept of a Jonsson set becomes clear. Moreover, in $[10,11]$, related to the concept of a certain dimension, various generalizations of the well-known model-theoretic results were obtained. The concept of dimension is the cornerstone of all mathematics, and therefore finding new implementations of the ideas of dimension is an urgent task.

On the other hand, one of the classical questions of the Model Theory is the question of the spectrum of models, that is, the number of models in a particular cardinality up to isomorphism. In the study of Jonsson theories, the concept of a Jonsson spectrum was considered [12]. In this case, we have some syntactic invariant of an arbitrary model of an arbitrary signature. Namely, the number of Jonsson theories whose model is the given model under consideration up to cosemanticness [13]. It turned out that within the framework of studying the Jonsson spectrum one can consider the well-known classical question: let $K$ be a certain class of models of an arbitrary signature $\sigma$ of a first-order language $L$ and $L_{0}$ is the set of all sentences of this language, i.e. $L_{0} \subset L$ and $\Gamma \subseteq L_{0}$. If we consider the $T h_{\Gamma}(K)$ theory of the class $K$, where $T h_{\Gamma}(K)=\{\phi \in \Gamma: \forall A \in K$ follows that $A=\phi\}$ and consider the class of models of this theory $M=\operatorname{Mod}\left(T h_{\Gamma} K\right)$, then the connection between the classes $M$ and $K$ is the classical formulation of the question of the axiomatizability of the class $K$. In the case when the theory is not complete (and Jonsson theories, generally speaking, are like that), this class of problems becomes difficult enough for a complete description. Another difficulty in the study of Jonsson theories is the fact that the well-described part is only a small fraction of the class of all Jonsson theories of the fixed signature. And

\footnotetext{
${ }^{*}$ Corresponding author.

E-mail: aibat.kz@gmail.com
} 
this part of the class in question consists of a union of classes consisting of perfect Jonsson theories. That is theories that have a model companion and their class of existentially closed models satisfy the above classical problem of axiomatizability of this class.

The concept of the central type is associated with the concept of hereditary enrichment in Jonsson's theory $[14,15]$. The concept of heredity at the moment does not have a complete description, except for some intuitive guesses. We took one of them from the ideas of constructing a pregeometry [16] on the Boolean of the semantic model of a fixed Jonsson theory. The next idea relates to the fact that in [17] it was noted that the saturation of any universal model of quasi-transcendental theory with a strong base, as well as, the superstability of such theories (Theorem 2).

In this paper, the notion of quasi-transcendence will be replaced by $\varphi(x)$-transcendence, where $\varphi(x)$ defines some Jonsson set $[13 ; 278]$, and the notion of a strong base is replaced by the notion of an essential base but in permissible enrichment of the hereditary Jonsson theory.

\section{An essential types and an essential base of central types}

Before we give the necessary definitions, we recall how we get the central type.

Let $L$ be a first-order language, $T$ be an arbitrary hereditary Jonsson theory in $L$ of the signature $\sigma, C$ be a semantic model of the theory $T, A \subseteq C, \sigma^{\prime}=\sigma \bigcup\{P\} \bigcup\{c\}$.

Let $\bar{T}=T \bigcup T h_{\forall \exists}\left(C, c_{a}\right)_{a \in A} \bigcup\{P(c)\} \bigcup\{P, \subseteq\}$, where $\{P, \subseteq\}$ is an infinite set of sentences expressing the fact that the interpretation of the symbol $P$ is an existentially closed submodel in the language of signature $\sigma^{\prime}$. That is, the interpretation of the symbol $P$ is a solution to the following equation $P(C)=M, M \in E_{T}$ in the language of the signature $\sigma^{\prime}$.

Since we know an example of the fact that the Jonsson theory in enrichment with a unary predicate does not preserve the property of amalgam, and the central type is obtained from the center of the Jonsson theory in the enriched language, we need the following definitions.

Definition 1. An enrichment $\bar{T}$ of the Jonsson theory $T$ is said to be permissible if any $\nabla$-type (it mean that $\nabla$ subset of language $L_{\sigma}$ and any formula from this type belongs to $\nabla$ ) in this enrichment is definable in the framework of $\bar{T}_{\Gamma \text {-stability. }}$

Definition 2. The Jonsson theory is said to be hereditary, if in any of its permissible enrichment, it preserves the Jonssonness.

Consider all extensions of the theory $\bar{T}$ in the language of signature $\sigma^{\prime}$. Since $T$ is a hereditary theory, then $\bar{T}$ will be a Jonsson theory, so it has a center, and we denote it by $\bar{T}^{*}$, and this center is equal to one of above completions theory $\bar{T}$. When restricting the signature $\sigma^{\prime}$ to $\sigma \cup\{P\}$, according to the laws of first-order logic, the constant $c$ no longer belongs to this signature, and we can replace this constant with a variable, for example, $x$. And then the theory $\bar{T}^{*}$ becomes a complete 1-type for the variable $x$. We will call this type the central type of the theory $\bar{T}$ in the above enrichment.

In what follows, we define the concept of an essential type and an essential base using the Rudin-Keisler order on the set of central types of some hereditary Jonsson theory in a permissible enrichment.

Definition 3. Type $p \in S_{\nabla}^{(1)}(X)$ is called essential if for any set $Y, Y \subseteq N, N \in E_{T}$, such that $X \subseteq Y$ in $T$ exists only unique type $q \in S_{\nabla}^{(1)}(Y)$ and the type $q$ is a $J$-nonforking extension of type $p$.

Let $p, q \in S_{\nabla}^{(1)}(X), \mathfrak{A} \in E_{T}$ and $X \subseteq A$. The relation $p \leq_{A} q$ is means that for any model $\mathfrak{B} \in E_{T}$, such that $\mathfrak{B} \supseteq \mathfrak{A}$, from the realizability of $q$ in $B \backslash A$ implies the realizability of $p$ in $B \backslash A$. The relation $p \equiv q$ means that for any model $\mathfrak{A} \in E_{T}, X \subseteq A$, has $p \leq_{A} q$ and $q \leq_{A} p$. We denote the set $\left\{q \mid q \in S_{\nabla}^{(1)}(X), p \equiv q\right\}$ by $[p]$, and the set $\left\{[p] \mid p \in S_{\nabla}^{(1)}(X)\right\}$ denote by $S_{\nabla}^{(1)}[X]$. We write $[p] \leq_{A}[q]$, if $p \leq_{A} q$. The types $p, q$ are called independent if for any $\mathfrak{A} \in E_{T}, X \subseteq A$, don't have a place neither $p \leq_{A} q$, nor $q \leq_{A} p$. If $p$ and $q$ are independent, then we say that $[p]$ and $[q]$ are independent.

The following definition gives the concept of a basis among the above types.

Definition 4. The set $B=\left\{\left[p_{i}\right] \in S_{\nabla}^{(1)}[X] \mid i \in I\right\}$ is called base for $S_{\nabla}^{(1)}[X]$ if:

(1) $\left[p_{i}\right]$ and $\left[q_{j}\right]$ independent for $i \neq j$;

(2) for any $[q] \in S_{\nabla}^{(1)}[X]$ and $\mathfrak{A} \in E_{T}, X \subseteq A$, exists $i \in I$, such that $\left[p_{i}\right] \leq_{A}[q]$.

Definition 5 . The base of the theory $T$ is the base for $S_{\nabla}^{(1)}[\varnothing]$ (if it exists). The base $B$ of $T$ is called essential if for any $[p] \in B$ exists an essential type $q \in[p]$.

Let us define the notion of pregeometry on the Boolean of subsets of the semantic model of a fixed Jonsson theory $T$. 
Let $T$ be a some Jonsson theory, $C$ be its semantic model.

Definition 6 . Let $C$ be as above and let cl: $P(C) \rightarrow P(C)$ be an operator on the power set of $C$. We say that $(C, c l)$ is a pregeometry if the following conditions are satisfied:

i) if $A \subseteq C$, then $A \subseteq \operatorname{cl}(A)$ and $\operatorname{cl}(\operatorname{cl}(A))=\operatorname{cl}(A)$.

ii) if $A \subseteq B \subseteq C$, then $\operatorname{cl}(A) \subseteq \operatorname{cl}(B)$.

iii)(exchange) if $A \subseteq C, a, b \in C$ and $a \in \operatorname{cl}(A \cup\{b\})$, then $a \in \operatorname{cl}(A), b \in \operatorname{cl}(A \cup\{a\})$.

iv)(finite character) if $A \subseteq C$ and $a \in \operatorname{cl}(A)$, then there is a finite $A_{0} \subseteq A$ such that $a \in \operatorname{cl}\left(A_{0}\right)$.

We say that $A \subseteq C$ is closed if $\operatorname{cl}(A)=A$.

In particular, we can define the notion of strong minimality on formula subsets of existentially closed submodels of the semantic model of a fixed Jonsson theory [13].

If $D$ is strongly minimal, we can associate a pregeometry by defining $\operatorname{cl}(A)=\operatorname{acl}(A) \cap D$ for $A \subseteq D$.

We can generalize basic ideas about independence and dimension from strongly minimal sets to arbitrary pregeometries for any subset of fix semantic model of some Jonsson theory.

Let as call $(X, c l$ )-Jonsson pregeometry (further $J$-pregeometry) if $X \subseteq C, C$ and $T$ as above.

Definition \%. If $(X, c l)$ is a Jonsson pregeometry, we say that $A$ is Jonsson independent if $a \notin \operatorname{cl}(A \backslash\{a\})$ for all $a \in A$ and that $B$ is a $J$-basis for $Y$ if $B \subseteq Y$ is $J$-independent and $Y \subseteq \operatorname{acl}(B)$.

Definition 8. We say that a $J$-pregeometry $(X, c l)$ is $J$-geometry if $c l(\emptyset)=\emptyset$ and $\operatorname{cl}(\{x\})=\{x\}$ for any $x \in X$.

If $(X, c l)$ is a $J$-pregeometry, then we can naturally define a $J$-geometry. Let $X_{0}=X \backslash \operatorname{cl}(\emptyset)$. Consider the relation $\sim$ on $X_{0}$ given by $a \sim b$ iff $\operatorname{cl}(\{a\})=\operatorname{cl}(\{b\})$. By exchange, $\sim$ is an equivalence relation. Let $\widehat{X}$ be $X_{0} / \sim$. Define $\widehat{c l}$ on $\widehat{X}$ by $\widehat{c l}(A / \sim)=\{b / \sim: b \in \operatorname{cl}(A)\}$.

Definition 9. Let $(X, c l)$ be $J$-pregeometry. We say that $(X, c l)$ is trivial if $c l(A)=Y_{a \in A} c l\{a\}$ for any $A \subseteq X$. We say that $(X, c l)$ is modular if for any finite-dimensional closed $A, B \subseteq X$

$$
\operatorname{dim}(A \cup B)=\operatorname{dim} A+\operatorname{dim} B-\operatorname{dim}(A \cap B)
$$

Definition 10 . We say that $(X, c l)$ is locally modular if $\left(X, c l_{a}\right)$ is modular for some $a \in X$.

Definition 11. If $X=C$ and $(X, c l)$ is a modular, then the Jonsson theory $T$ is called modular.

Let in what follows the operator $c l$ is either an algebraic or a definable closure of some $\varphi(x)$-set, that is, if $C$ is a semantic model of some Jonsson theory, and $\varphi(C)=A$, then $\operatorname{cl}(A)=M \in E_{T}, M=M_{1} \cup M_{2}, M_{1}=a c l(A)$, $M_{2}=\operatorname{dcl}(A)$.

Definition 12. Let $X \subseteq C$. We will say that a set $X$ is $\nabla$-cl-Jonsson subset of $C$, if $X$ satisfies the following conditions:

1) $X$ is $\nabla$-definable set (this means that there is a formula from $\nabla$, the solution of which in the $C$ is the set $X$, where $\nabla \subseteq L$, that is $\nabla$ is a view of formula, for example $\exists, \forall, \forall \exists$ and so on.);

2) $c l(X)=M, M \in E_{T}$, where $c l$ is some closure operator defining a pregeometry over $C$ (for example $c l=a c l$ or $c l=d c l)$.

It is clear that the $\varphi(x)$-Jonsson set is a special case of Definition 12, i.e. these are elements of the semantic model that are solutions of the formula $\varphi(x)$ in the model $C$.

And in particular, $\varphi(x)$-set is called a $\varphi(x)$-Jonsson set in the theory $T$ if the following definition holds.

Definition 13. Let $X \subseteq C$. We will say that a set $X$ is $\varphi(x)$-Jonsson subset of $C$, if $X$ satisfies the following conditions:

1) $X$ is $\varphi(x)$-definable set (this means that there is a formula from $\varphi(x)$, the solution of which in the $C$ is the set $X$, where $\varphi(x) \subseteq L$, that is $\varphi(x)$ is a view of formula $\exists$ );

2) $\operatorname{cl}(X)=M, M \in E_{T}$, where $c l$ is some closure operator defining a pregeometry over $C$ (for example $c l=a c l$ or $c l=d c l)$.

Any subset of the semantic model is called the set $X$ in the Jonsson theory $T$.

\section{The atomicity and the primeness of $\varphi(x)$-sets}

A model-theoretical property of a model is said to be an $A P A$-property if it satisfies the properties of algebraic primeness and atomicity in the sense of [18]. For example, let us give an important definition of $A P A$-transcendence.

Definition 14. A set $X$ is called an $A P A$-set if it is $\varphi(x)$-Jonsson set and $M$ is algebraically prime and $\left(\Sigma_{1}, \Sigma_{1}\right)$-atomic model, where $M=\operatorname{cl}(X), M \in E_{T}$. And in this case the model $M$ is called an $A P A$-existentially closed model. 
And accordingly, an analog of the quasi-transcendental theory from [17] is the notion of the $A P A$-transcendental theory.

Definition 15. A Jonsson theory $T$ is called $A P A$-transcendental, if for any $A P A$-set of the $X$ in the theory $T$ there exist $A P A$-existentially closed model $M$, such that $\operatorname{cl}(X)=M$.

We recall the classical definition of a convex theory belongs to A. Robinson.

Definition 16. A theory $T$ is called convex if for any its model $A$ and for any family $\left\{B_{i} \mid i \in I\right\}$ of substructures of $A$, which are models of the theory $T$, the intersection $\bigcap_{i \in I} B_{i}$ is a model of $T$, provided it is non-empty. If in addition such an intersection is never empty, then $T$ is called strongly convex.

The following definition selects a rather interesting subclass in the class of convex Jonsson theories, which is defined by the closure of some formula $\varphi(x)$.

Definition 17. The theory $T$ will be called $\varphi(x)$-convex if:

1) it is convex in the classical sense;

2) for any existentially closed model $N$ of this theory, there is a theoretical set $A$ such that $\operatorname{cl}(A)=\cap_{i} B_{i}$, $B_{i} \prec_{\Sigma_{1}} N$ and exists $\varphi(x): \varphi(C)=A$.

Let $C$ be semantic model, $M_{i} \prec C, i \in I$. There are 2 possible cases.

1 case. $M_{i}$ are existentially closed models.

2 case. $M_{i}$ are not existentially closed models. Then, by the Lowenheim-Sculem up theorem, there exist elementary extensions $M_{i}^{\prime} \succ M_{i}$, which, according to Proposition $8.12[2 ; 97]$ will already be existentially closed models.

This means that in any case, there are models $M_{i}$ that will be existentially closed.

Summarizing the above, we can consider a more general situation, defining the next class of theories.

Definition 18. Let $T$ be the Jonsson theory. $T$ is called the $\varphi(x)$-transcendental if on any $\varphi(x)$-set there is $\left(\nabla_{1}, \nabla_{2}\right)$-atomic and $h$-prime model, where $\nabla_{1}, \nabla_{2} \subset L$ and $h$ is an arbitrary homomorphism between the models of the theory $T$.

We denote $\operatorname{Th}{ }_{\forall \exists}(M)$ by $\operatorname{Fr}(A)$ and call it a fragment of the Jonsson theory $T$, where $C$ is the semantic model of this theory, $A \subseteq C, M=\operatorname{cl}(A), M \in E_{T}$.

The following theorem is the main result of this article. This theorem shows that the number of fragments obtained under a closure of an algebraic or definable type does not exceed the number of homogeneous models of some Jonsson theory, which is obtained as a result of a permissible enrichment of the hereditary Jonsson theory.

Theorem. Let $T$ be hereditary, complete for $\exists$-sentences, $\varphi(x)$-convex, $\varphi(x)$-transcendental Jonsson theory with an essential base of central types in its fragments, where $\varphi(x)$ defines a $A P A$-subset of the semantic model $C$. Then there is a cardinal $\mu$ such that the number of fragments is determined by the following formula

$$
H(\alpha, \bar{T})= \begin{cases}1, & \text { at condition } I=1 \& \alpha>0 \\ \aleph_{0}^{\min \left(\mu, \aleph_{\alpha}\right)}, & \text { in other cases. }\end{cases}
$$

where $H(\alpha, \bar{T})$ is the number of homogeneous models of the theory $\bar{T}$.

Proof. Consider the enrichment of the language of the theory $T$ with a new constant symbol $c$ and a new unary predicate symbol $p$. Let us write one of the completions of the theory $T$ in the following form:

$$
\bar{T}=T \bigcup T h_{\forall \exists}\left(C, c_{a}\right)_{a \in A} \bigcup\{P(c)\} \bigcup\{P, \subseteq\} .
$$

Let $\{P, \subseteq\}$ be the set of sentences defining the $N$ model,where $N \in E_{T}, N$ is the core, $\left(\Sigma_{1}, \Sigma_{1}\right)$-atomic model. Such a model exists due to $\varphi(x)$-convexity of the theory $T$. Consider all existentially closed extensions of the model $N$. It is easy to see that the class of all these extensions does not exceed $E_{T}$. Moreover, due to the convexity of the theory $T$, this class coincides with $E_{T}$. To count the number of models $E_{T}$, we will use the central type technique. Let $M_{i}$ be an arbitrary existentially closed extension of the model $N$. Consider the central type of models $M_{i}$. To do this, we will consider the fragments $T h_{\forall \exists}\left(M_{i}\right)$, denote them $T_{i}$. This is a Jonsson theory in the language of the theory $T$, since $M_{i} \in E_{T}$. Consider

$\bar{T}_{M_{i}}=T \bigcup T h_{\forall \exists}\left(C_{\uparrow M_{i}}, c_{a}\right)_{a \in A} \bigcup\{P(c)\} \bigcup\left\{P_{M_{i}}, \subseteq\right\}$, where $\left\{P_{M_{i}}, \subseteq\right\}$ is a set sentences reflecting the fact that $N \preceq \Sigma_{1} M_{i}$. Due to the heredity of the $T_{i}$ theory, the theory $\bar{T}_{M_{i}}$ is a Jonsson theory, and it has a corresponding center, we denote it $\bar{T}_{M_{i}}^{*}$. By virtue of the laws of first-order logic, we can replace the constant symbol $c$ with the symbol of the variable $x$, if in the new language we will leave only the predicate symbol $P$. Then instead of the center $\bar{T}_{M_{i}}^{*}$ we get some complete type in the old language with the predicate symbol $P$. Let's denote this type by $p_{M_{i}}^{c}$. This is the central type of the theory $\bar{T}_{M_{i}}$. 
There will be as many such central types as there are $M_{i}$ models. On this set of central types, i.e. obtained for each model $M_{i} \in E_{T}$, we consider the essential base of essential central types. And concerning it we use the results of Theorems 1 and 2 from [17]; in the language of central types, this means that each $T_{i}$ is a perfect Jonsson theory, since Theorem 2 proved the saturation of the universal model for a quasi-transcendental theory with a strong base. Each semantic model of Jonsson theory is universal in the sense of isomorphic embedding and relative to $\exists$-formulas, and due to the $\exists$-completeness of the $T$ theory, we can transfer the proof of saturation for $\exists$-types. This means that the theories $T_{i}$ are perfect Jonsson theories, due to heredity, $\bar{T}_{M_{i}}$ are also perfect Jonsson theories, so their centers are model complete, which means that all embeddings between models are elementary. In particular, all algebraically prime models are prime models, and $\left(\Sigma_{1}, \Sigma_{1}\right)$-atomic models are atomic models of the theory $\bar{T}_{M_{i}}^{*}$. Therefore, there will be as many central types as there are semantic models of theories $T_{i}$, but all these models are existentially closed models, like the semantic models of Jonsson theories. By virtue of the perfectness of Jonsson's theory $\overline{T_{i}}$, the class $E_{\overline{T_{i}}}$ is equivalent to the class $\operatorname{Mod} \bar{T}_{i}^{*}$. Because of the above, we can conclude that the number of central types for the Jonsson theory is exactly equal to the number of universally homogeneous models, and we can apply the result of Theorem 6 from [17], concerning only the spectrum of homogeneous models, which uniquely determines the criterion for the Jonsson theory, namely, a theory is Jonsson if and only if it has a universally homogeneous model of sufficient power.

\section{Acknowledgments}

This work was supported by the Science Committee of the Ministry of Education and Science of the Republic of Kazakhstan (grant AP09260237).

\section{References}

1 Kueker D.W. Core structures for theories / D.W. Kueker // Fundamenta Mathematicae LXXXIX. 1973. - P. 154-171.

2 Barwise J.Ed., Handbook of mathematical logic. - Part 1, Model Theory / J. Barwise. - Moscow: Science, 1982.

3 Yeshkeyev A.R. del-cl-atomic and prime sets / A.R. Yeshkeyev, A.K. Issayeva // Bulletin of the Karaganda University - Mathematics. — 2019. — 93. — № 1. - P. 88-94. DOI: 10.31489/2019M1/88-94

4 Yeshkeyev A.R. The Properties of Similarity for Jonsson's Theories and Their Models / A.R. Yeshkeyev // Bulletin of the Karaganda University. Mathematics series. - 2015. — 80. — № 4. - P. 52-59.

5 Yeshkeyev A.R. The atomic definable subsets of semantic model / A.R. Yeshkeyev, N.M. Mussina, A.K. Issayeva // Bulletin of the Karaganda University. Mathematics series. - 2019. - 94. - № 2. P. 84-91. DOI 10.31489/2019M2/84-91

6 Yeshkeyev A.R. Properties of hybrids of Jonsson theories/ A.R. Yeshkeyev, N.M. Mussina // Bulletin of the Karaganda University. Mathematics series. — 2018. - 92. — № 4. - P. 99-104. DOI: 10.31489/2018M4 /99-104

7 Yeshkeyev A.R. The properties of central types with respect to enrichment by Jonsson set / A.R. Yeshkeyev // Bulletin of the Karaganda University. Mathematics series. — 2017. — 85. — № 1. P. 36-40. DOI: $10.31489 / 2017 \mathrm{M} 1 / 36-40$

8 Yeshkeyev A.R. Companions of the fragments in the Jonsson enrichment / A.R. Yeshkeyev // Bulletin of the Karaganda University. Mathematics series. — 2017. — 85. — № 1. - P. 41-45. DOI: 10.31489/2017M1 /41-45

9 Yeshkeyev A.R. Companions of $(\mathrm{n}(1), \mathrm{n}(2))$-Jonsson theory / A.R. Yeshkeyev, M.T. Omarova // Bulletin of the Karaganda University. Mathematics series. - 2019. - 96. - № 4. - P. 75-80. DOI 10.31489/2019M4 75-80

10 Ешкеев A.P. JSp-косемантичность и $J S B$-свойство абелевых групп [Электронный ресурс] / A.Р. Ешкеев, О.И. Ульбрихт // Siberian Electronic Mathematical Reports. - 2016. - 13. - C. 861-874. Режим доступа: http://semr.math.nsc.r. Doi: 10.17377/semi.2016.13.068

11 Ешкеев A.P. JSp-косемантичность $R$-модулей [Электронный ресурс] / A.Р. Ешкеев, О.И. Ульбрихт // Siberian electronics mathematical reports. - 2019. - 16. - C. 1233-1244. Режим доступа: http: //semr.math.nsc.ru DOI: 10.33048/semi.2019.16.084 
12 Yeshkeyev A.R. Model-theoretical questions of the Jonsson spectrum / A.R. Yeshkeyev // Bulletin of the Karaganda University. Mathematics series. - 2020. - 98. — № 2. - P. 165-173. DOI: 10.31489/2020M2 /165-173

13 Ешкеев А.Р. Йонсоновские теории и их классы моделей: моногр. / А.Р. Ешкеев, М.Т. Касыметова. - Караганда: Изд-во КарГУ, 2016. - 370 с.

14 Yeshkeyev A.R. The J-minimal sets in the hereditary theories / A.R. Yeshkeyev, M.T. Omarova, G.E. Zhumabekova // Bulletin of the Karaganda University. Mathematics series. - 2019. - 94. - № 2 . - P. 92-98. DOI 10.31489/2019M2/92-98

15 Yeshkeyev A.R. Central types of convex fragments of the perfect Jonsson theory / A.R. Yeshkeyev, M.T. Omarova // Bulletin of the Karaganda University. Mathematics series. - 2019. - 93. - № 1 . P. 95-101. DOI 10.31489/2019M1/95-101

16 Yeshkeyev A.R. Small models of hybrids for special subclasses of Jonsson theories / A.R. Yeshkeyev, N.M. Mussina // Bulletin of the Karaganda University. Mathematics series. — 2019. — 95. - № 3. P. 68-73. DOI 10.31489/2019M2/68-73

17 Мустафин Т.Г. О сильной базе элементарных типов теорий / Т.Г. Мустафин // Сиб. матем. журн. - 1977. - 18. - № 6. - C. 1356-1966.

18 Baldwin J.T. Algebraically prime models / J.T. Baldwin, D.W. Kueker // Ann. Math. Logic. - 1981. 20. - P. 289-330.

\title{
А.Р. Ешкеев, М.Т. Омарова
}

\section{Дөңес теорияның централдық типтерінің елеулі базасы}

\begin{abstract}
Мақалада дөңес теорияның централдық типтерінің елеулі базасының модельді-теоретикалық қасиеттері қарастырылған. Рұқсат етілген байытылған сигнатурада центр мен йонсондық теорияның байланысы көрсетілген. Сонымен қатар қарастырылып отырған теориялар мұралы теориялар болып табылады. Осы жұмыс 2 параграфқа бөлінген: 1) елеулі типтер және централдық типтердің елеулі базасы (сонымен бірге елеулі тип пен елеулі база ұғымдары Рудин-Кейслердің реті арқылы анықталады, яғни кейбір мұралы йонсондық теорияның рұқсат етілген байытуында централдық типтердің жиынында); 2) $\varphi(x)$ - жиынның атомдығы және жайлылығы. Келесі жаңа ұғымдар енгізілген: $\varphi(x)$ - йонсондық жиын, $A P A-$ жиын, $A P A-$ экзистенционалды тұйық модель, $\varphi(x)-$ дөңес теория, $\varphi(x)$ - трансцендентті теория, $A P A-$ трансцендентті теория. Осы мақаланың бір идеясы Т.Ғ. Мұстафиннің еңбегінде квазитрансцендентті теорияның кез-келген әмбебап моделі қатты базамен қаныққан екендігі деп атап өтілген деген дерекке қатысты, бірақ мақала авторлары бұл нәтижені ескере отырып жалпылау жасады: квазитрансценденттілік ұғымы $\varphi(x)$-трансценденттілікке ауыстырылды, мұндағы $\varphi(x)$ кейбір йонсондық жиынды анықтайды; және қатты база ұғымы елеулі база ұғымымен алмастырылды, бірақ мұралы йонсондық теорияның рұқсат етілген байытуында. Жұмыстың негізгі нәтижесі көрсеткендей, алгебралық немесе анықталатын типтің тұйықталуы кезінде алынған фрагменттер саны мұралы йонсондық теорияның рұқсат етілген байыту нәтижесінде алынған кейбір йонсондық теорияның біртекті модельдерінің санынан аспайды.
\end{abstract}

Kiлm сөздер: йонсондық теория, централдық типтер, елеулі база, $\varphi(x)$-жиын, $\varphi(x)$-дөңес теория, $\varphi(x)$ трансцендентті теория, $A P A$-трансцендентті теория, фрагмент.

\section{А.P. Ешкеев, М.Т. Омарова}

\section{Существенная база центральных типов выпуклой теории}

В статье рассмотрены теоретико-модельные свойства существенной базы центральных типов выпуклой теории. Показаны связи между центром и йонсоновской теорией в разрешенном обогащении сигнатуры. При этом рассматриваемые теории являются наследственными. Данная работа разбита на 2 параграфа: 1) существенные типы и существенная база центральных типов (при этом понятия существенного типа и существенной базы определяются с помощью порядка Рудина-Кейслера на 
множестве центральных типов некоторой наследственной йонсоновской теории в разрешенном обогащении); 2) атомность и простота $\varphi(x)$-множеств. Введены новые понятия: $\varphi(x)$ - йонсоновское множество; $A P A-$ множество; $A P A$ - экзистенциально замкнутая модель; $\varphi(x)$ - выпуклая теория; $\varphi(x)$ - трансцендентная теория; $A P A$ - трансцендентная теория. Одна из идей данной статьи относится к тому факту, что в работе Т.Г. Мустафина было замечено, что любая универсальная модель квазитрансцендентной теории с сильной базой насыщена. Авторы статьи обобщили этот результат с учетом того, что понятие квазитрансцендентности будет заменено $\varphi(x)$-трансцендентностью, где $\varphi(x)$ задает некоторое йонсоновское множество; а понятие сильной базы заменено понятием существенной базы, но в разрешенном обогащении наследственной йонсоновской теории. Главный результат данной работы показывает, что число фрагментов, полученных под замыканием алгебраического или определимого типа, не превосходит числа однородных моделей некоторой йонсоновской теории, которая получается в результате разрешенного обогащения наследственной йонсоновской теории.

Ключевые слова: йонсоновская теория, центральные типы, существенная база, $\varphi(x)$-множество, $\varphi(x)$ выпуклая теория, $\varphi(x)$-трансцендентная теория, $A P A$-трансцендентная теория, фрагмент.

\section{References}

1 Kueker, D.W. (1973). Core structures for theories. Fundamenta Mathematicae LXXXIX, 154-171.

2 Barwise, J. (1982). Ed., Handbook of mathematical logic. Part 1, Model Theory. Moscow, Science.

3 Yeshkeyev, A.R., \& Issayeva, A.K. (2019). del-cl-atomic and prime sets. Bulletin of the Karaganda University - Mathematics, 93(1), 88-94. DOI: 10.31489/2019M1/88-94

4 Yeshkeyev, A.R. (2015). The Properties of Similarity for Jonsson's Theories and Their Models. Bulletin of the Karaganda University - Mathematics, 80(4), 52-59.

5 Yeshkeyev, A.R., Issayeva, A.K., \& Mussina, N.M. (2019). The atomic definable subsets of semantic model. Bulletin of the Karaganda University - Mathematics, 94(2), 84-91.

DOI 10.31489/2019M2/84-91

6 Yeshkeyev, A.R., \& Mussina, N.M. (2018). Properties of hybrids of Jonsson theories. Bulletin of the Karaganda University - Mathematics, 92(4), 99-104. DOI: 10.31489/2018M4/99-104

7 Yeshkeyev, A.R. (2017). The properties of central types with respect to enrichment by Jonsson set. Bulletin of the Karaganda University - Mathematics, 85(1), 36-40. DOI: 10.31489/2017M1/36-40

8 Yeshkeyev, A.R. (2017). Companions of the fragments in the Jonsson enrichment. Bulletin of the Karaganda University - Mathematics, 85(1), 41-45. DOI: 10.31489/2017M1/41-45

9 Yeshkeyev, A.R., \& Omarova, M.T. (2019). Companions of $(\mathrm{n}(1), \mathrm{n}(2))$-Jonsson theory. Bulletin of the Karaganda University - Mathematics, 96(4), 75-80. DOI 10.31489/2019M4/75-80

10 Yeshkeyev, A.R., \& Ulbrikht, O.I. (2016). JSp-kosemantichnost i $J S B$-svoistvo abelevykh hrupp [JSp-cosemanticness and JSB property of Abelian groups]. Sibirskie elektronnye matematicheskie izvestiia - Siberian Electronic Mathematical Reports, 13, 861-874 [in Russian].

11 Yeshkeyev, A.R., \& Ulbrikht, O.I. (2019). JSp-kosemantichnost $R$-modulei [JSp-cosemanticness of R-modules]. Sibirskie elektronnye matematicheskie izvestiia - Siberian Electronic Mathematical Reports, 16, 1233-1244. Doi: 10.33048/semi.2019.16.084 [in Russian].

12 Yeshkeyev, A.R. (2020). Model-theoretical questions of the Jonsson spectrum. Bulletin of the Karaganda University - Mathematics, 98(2), 165-173. DOI: 10.31489/2020M2/165-173

13 Yeshkeyev, A.R., \& Kassymetova, M.T. (2016). Yonsonovskie teorii i ikh klassy modelei /Jonsson theories and their classes of models]. Karaganda: Izdatelstvo Karahandinskoho universiteta [in Russian].

14 Yeshkeyev, A.R., Omarova, M.T., \& Zhumabekova, G.E. (2019). The J-minimal sets in the hereditary theories. Bulletin of the Karaganda University - Mathematics, 94 (2), 92-98. DOI 10.31489/2019M2/92-98

15 Yeshkeyev, A.R., \& Omarova, M.T. (2019). Central types of convex fragments of the perfect Jonsson theory Bulletin of the Karaganda University - Mathematics, 93(1), 95-101.

DOI $10.31489 / 2019 \mathrm{M} 1 / 95-101$

16 Yeshkeyev, A.R., \& Mussina, N.M. (2019). Small models of hybrids for special subclasses of Jonsson theories Bulletin of the Karaganda University - Mathematics, 95(3), 68-73.

DOI $10.31489 / 2019 \mathrm{M} 2 / 68-73$ 
17 Mustafin, T.G. (1977). O silnoi baze elementarnykh tipov teorii [On a strong base of elementary types of theories]. Sibirskii matematicheskii zhurnal, 18(6), 1356-1966 [in Russian].

18 Baldwin, J.T., \& Kueker, D.W. (1981). Algebraically prime models. Ann. Math. Logic., 20, 289-330. 


\author{
T.K. Yuldashev*, B.I. Islomov, U.Sh. Ubaydullaev \\ National University of Uzbekistan, Tashkent, Uzbekistan \\ (E-mail: tursun.k.yuldashev@gmail.com, islomovbozor@yandex.com,ulugbekuz88@mail.ru)
}

\title{
On Boundary Value Problems for a Mixed Type Fractional Differential Equation with Caputo Operator
}

\begin{abstract}
This article is devoted to study the boundary value problems of the first and second kind with respect to the spatial variable for a mixed inhomogeneous differential equation of parabolic-hyperbolic type with a fractional Caputo operator in a rectangular domain. In the study of such boundary value problems, we abandoned the boundary value condition with respect to the first argument and instead it is used additional gluing condition. In this case, in the justification of the unique solvability of the problems, the conditions on the boundary domain are removed. This allowed us to weaken the criterion for the unique solvability of boundary value problems under consideration. The solution is constructed in the form of Fourier series with eigenfunctions corresponding to homogeneous spectral problems. Estimates for the convergence of Fourier series are obtained as a regular solution of this mixed equation.
\end{abstract}

Keywords: Mixed differential equation, fractional order, Caputo operator, non model equation, Fourier series, gluing conditions, unique solvability.

\section{Introduction}

The theory of boundary value problems for differential equations of mixed parabolic-hyperbolic and elliptichyperbolic types, by virtue of its applied and theoretical significance, in recent years has become one of the most important branches of the theory of partial differential equations. In 1940, F. I. Frankl discovered applications of the Tricomi problem for the Chaplygin equation in transonic gas dynamics. Later the new applications of mixed-type equations have been found in the theory of Laval nozzles, in plasma theory, and in other branches of physics and mechanics.

Mathematicians began to study more often by the method of Fourier series the unique solvability and stability of the solution of the Dirichlet and Tricomi problems for a mixed type model differential equations of the second order

$$
0=\left\{\begin{array}{c}
u_{t}-u_{x x}+b^{2} u, t \geq 0, \\
u_{t t}-u_{x x}+b^{2} u, t<0
\end{array}\right.
$$

in rectangle domain $\Omega=\{(t, x):-p<t<q, 0<x<l\}$. We note that in [1-3] for this kind of equations in the rectangular domain with two gluing conditions and with a condition over the entire boundary domain were studied. In studying the unique solvability of Dirichlet and Tricomi problems for this kind of mixed equations there is a condition to the measure of the boundary domain. Our approach interfered with the global solvability of the considering problem in an arbitrary rectangle. The method of Fourier series is also widely used in the works of other authors in the study of local and nonlocal boundary value problems for differential and integro-differential equations (see, for example, works [4-9]). The problem of the correct choice of boundary value conditions for a wide class of singular partial differential equations are solved in [10]. The aggregated theorems of existence and uniqueness of classical solutions can be proved with continuously depending of experimental definite function. In [11] a nonlocal problem for the fourth order system of loaded partial differential equations is considered and the questions of a existence unique solution of the considered problem and ways of its construction are investigated.

The fractional differential and integral operators have applications in many fields of mathematical physics, engineering, neurobiology, economics, control theory and combustion science $[12,13]$. Therefore, this kind of differential and integral operators plays an important role in the theory of linear and nonlinear analysis. It is also

\footnotetext{
${ }^{*}$ Corresponding author.

E-mail: tursun.k.yuldashev@gmail.com
} 
known that in control theory is obtained a dynamic system, describing by the aid of fractional order differential equations [14]. There are classical methods of solving some kind of fractional differential equations with RiemannLiouville operator, with the Caputo operator, or with the Erdeli-Kober operator. The Cauchy problems for the diffusion-wave equation with fractional differentiation operators in the sense of Riemann-Liouville and Caputo were investigated in [15-19]. Such kind of problems are of great importance in the construction of mathematical models of diffusion processes. Interesting results were obtained in works [20-22] for the fractional partial differential equations.

In this paper in the rectangular domain the unique solvability of the problem with boundary conditions first and second kind with respect to the spatial variable is established for an inhomogeneous parabolic-hyperbolic equation with fractional Caputo operator. In studying this boundary value problem application of three gluing conditions allowed us to solve the problem in arbitrary rectangular domain. This work is a further development of work [23] for the case of an inhomogeneous equation.

So, in rectangle domain $\Omega=\{(t, x):-p<t<q, 0<x<l\}$ we consider a differential equation of mixed parabolic-hyperbolic type

$$
\left\{\begin{array}{c}
{ }_{c} D_{0 t}^{\alpha} U-U_{x x}+\lambda^{2} U=f(t, x), \quad t \geq 0 \\
U_{t t}-U_{x x}+\lambda^{2} U=f(t, x), \quad t<0
\end{array}\right.
$$

where $\lambda \geq 0, l>0, p>0, q>0$ are known real numbers, $f(t, x)$ is known function and ${ }_{c} D_{0 t}^{\alpha}$ is fractional order operator in the sense of Caputo:

$$
{ }_{c} D_{0 t}^{\alpha} g(t)=\left\{\begin{array}{l}
\frac{1}{\Gamma(1-\alpha)} \int_{0}^{t}(t-z)^{-\alpha} g^{\prime}(z) d z, 0<\alpha<1, \\
\frac{d}{d t} g(t), \quad \alpha=1,
\end{array}\right.
$$

$\Gamma(1-\alpha)$ is Euler gamma function.

Note that the Caputo operator (2) can be represented as follows:

$$
{ }_{c} D_{0 t}^{\alpha} U(t, x)=I_{0 t}^{1-\alpha} \frac{\partial U(t, x)}{\partial t},
$$

where

$$
I_{a t}^{\alpha} g_{0}(t)=\frac{1}{\Gamma(\alpha)} \int_{a}^{t}(t-z)^{\alpha-1} g_{0}(z) d z
$$

is fractional order Riemann-Liouville integral.

We introduce the notations: $J=\{(t, x): t=0,0<x<l\}, \Omega=\Omega_{1} \cup \Omega_{2} \cup J$,

$$
\Omega_{1}=\Omega \cap\{(t, x): t>0, x>0\}, \Omega_{2}=\Omega \cap\{(t, x): t<0, x<0\} .
$$

In the domain $\Omega$ we consider the following problem:

Problem 1. It is required to find a function $U(t, x)$ with the following properties:

$$
\begin{gathered}
U(t, x) \in C(\bar{\Omega}) \cap C^{1}\left(\Omega_{2} \cup J\right), \quad t^{1-\alpha} U_{t}(t, x), t^{2-\alpha} U_{t t}(t, x) \in C\left(\Omega_{1} \cup J\right) ; \\
U_{t t} \in C\left(\Omega_{2} \cup J\right), \quad U_{x x} \in C\left(\Omega_{1} \cup \Omega_{2}\right),{ }_{c} D_{0 t}^{\alpha} U \in C\left(\Omega_{1} \cup J\right)
\end{gathered}
$$

and satisfies the equation (1) in the domains $\Omega_{j}(j=1,2)$; on the line $J$ satisfies the gluing conditions

$$
\begin{gathered}
U(+0, x)=U(-0, x), \quad(0, x) \in \bar{J}, \\
\lim _{t \rightarrow+0} t^{1-\alpha} U_{t}(t, x)=\lim _{t \rightarrow-0} U_{t}(t, x), \quad(0, x) \in J, \\
\lim _{t \rightarrow+0} t^{2-\alpha} U_{t t}(t, x)=\lim _{t \rightarrow-0} U_{t t}(t, x), \quad(0, x) \in J ;
\end{gathered}
$$

satisfies the following boundary value conditions

$$
U(t, 0)=0, \quad U(t, l)=0, \quad-p \leq t \leq q .
$$

So, we note that in studying this problem we use three gluing conditions $(5)-(7)$. 
Solutions of the equation (1), satisfying zero boundary value conditions (8), are sought in the form of the Fourier series

$$
U(t, x)=\sum_{n=1}^{\infty} u_{n}(t) \vartheta_{n}(x),
$$

where

$$
\begin{gathered}
u_{n}(t)=\int_{0}^{l} U(t, x) \vartheta_{n}(x) d x \\
\vartheta_{n}(x)=\sqrt{\frac{2}{l}} \sin \rho_{n} x, \rho_{n}=\sqrt{\mu_{n}^{2}+\lambda^{2}}, \quad \mu_{n}=\frac{\pi n}{l}, n \in \mathbb{N} .
\end{gathered}
$$

It is known that a system of functions $\left\{\vartheta_{n}(x)\right\}_{n=1}^{\infty}$ form a complete system of orthonormal functions in the space $L_{2}[0, l]$. We also expand the function $f(t, x)$ in a Fourier series by eigenvalue functions $\vartheta_{n}(x)$ :

$$
f(t, x)=\sum_{n=1}^{\infty} f_{n}(t) \vartheta_{n}(x),
$$

where

$$
f_{n}(t)=\int_{0}^{l} f(t, x) \vartheta_{n}(x) d x .
$$

Uniqueness of the solution of the problem 1

Theorem 1. If there exists a solution of the problem 1 , then this solution is unique.

Proof. Let $f(t, x)=0$ be in $\bar{\Omega}$. We prove that a homogeneous problem $U_{I}$ has only a trivial solution. We consider the function

$$
\theta_{m}(t)=\int_{0}^{l} U(t, x) \vartheta_{m}(x) d x .
$$

Then for the homogeneous equation (1) we obtain

$$
\begin{gathered}
{ }_{c} D_{0 t}^{\alpha} \theta_{m}(t)=\sqrt{\frac{2}{l}} \int_{0}^{l}\left[{ }_{c} D_{0 t}^{\alpha} U(t, x)\right] \sin \rho_{m} x d x=\sqrt{\frac{2}{l}} \int_{0}^{l} U_{x x}(t, x) \sin \rho_{m} x d x- \\
-\sqrt{\frac{2}{l}} \int_{0}^{l} \lambda^{2} U(t, x) \sin \rho_{m} x d x, \quad 0<t<q, \\
\theta_{m}^{\prime \prime}(t)=\sqrt{\frac{2}{l}} \int_{0}^{l} U_{t t}(t, x) \sin \rho_{m} x d x=\sqrt{\frac{2}{l}} \int_{0}^{l} U_{x x}(t, x) \sin \rho_{m} x d x- \\
-\lambda^{2} \sqrt{\frac{2}{l}} \int_{0}^{l} U(t, x) \sin \rho_{m} x d x, \quad-p<t<0 .
\end{gathered}
$$

Hence, integrating by parts two times over $x$ and taking the conditions (5)-(7) into account, we obtain

$$
\begin{gathered}
{ }_{c} D_{0 t}^{\alpha} \theta_{m}(t)+\rho_{m}^{2} \theta_{m}(t)=0, \quad 0<t<q, \\
\theta_{m}^{\prime \prime}(t)+\rho_{m}^{2} \theta_{m}(t)=0, \quad-p<t<0,
\end{gathered}
$$

Applying the gluing conditions (5)-(7) to (11), we obtain

$$
\theta_{m}(+0)=\int_{0}^{l} U(+0, x) \vartheta_{m}(x) d x=
$$




$$
\begin{gathered}
=\int_{0}^{l} U(-0, x) \vartheta_{m}(x) d x=\theta_{m}(-0), \\
\lim _{t \rightarrow+0} t^{1-\alpha} \theta_{m}^{\prime}(t)=\lim _{t \rightarrow+0} \int_{0}^{l} t^{1-\alpha} U_{t}(t, x) \vartheta_{m}(x) d x= \\
=\lim _{t \rightarrow-0} \int_{0}^{l} U_{t}(t, x) \vartheta_{m}(x) d x=\lim _{t \rightarrow-0} \theta_{m}^{\prime}(t), \\
\lim _{t \rightarrow+0} t^{2-\alpha} \theta_{m}^{\prime \prime}(t)=\lim _{t \rightarrow+0} \int_{0}^{l} t^{2-\alpha} U_{t t}(t, x) \vartheta_{m}(x) d x= \\
=\lim _{t \rightarrow-0} \int_{0}^{l} U_{t t}(t, x) \vartheta_{m}(x) d x=\lim _{t \rightarrow-0} \theta_{m}^{\prime \prime}(t) .
\end{gathered}
$$

By virtue of (3) and (4), the countable systems of differential equations (12) and (13), respectively, have general solutions

$$
\begin{gathered}
\theta_{m}(t)=c_{m} E_{\frac{1}{\alpha}}\left(-\rho_{m}^{2} t^{\alpha}, 1\right), \quad 0<t<q, \\
\theta_{m}(t)=a_{m} \cos \rho_{m} t+b_{m} \sin \rho_{m} t, \quad-p<t<0,
\end{gathered}
$$

where $a_{m}, b_{m}, c_{m}$ are arbitrary constants and $E_{\frac{1}{\alpha}}(z, 1)$ is the Mittag-Leffler function with the form:

$$
E_{\alpha, \sigma}(z) \equiv E_{\frac{1}{\alpha}}(z, \sigma)=\sum_{i=0}^{\infty} \frac{z^{i}}{\Gamma(\alpha i+\sigma)}, \quad \sigma>0 .
$$

Substituting (17) and (18) into (14)-(16) and taking into account the property of the Mittag-Leffler function

$$
E_{\frac{1}{\alpha}}(z)=1+z E_{\frac{1}{\alpha}}(z, \alpha+1)
$$

we obtain

$$
c_{m}=a_{m}, \quad b_{m}=-\frac{\rho_{m}}{\Gamma(\alpha)} c_{m}, \quad\left[\frac{(1-\alpha)}{\Gamma(\alpha)}+1\right] c_{m}=0 .
$$

Hence, we find that $c_{m}=a_{m}=b_{m}=0$. Consequently,

$$
\theta_{m}(t)=\int_{0}^{l} U(t, x) \vartheta_{m}(x) d x=0, t \in[-p, q] .
$$

Therefore, by virtue of completeness of the systems of eigenfunctions $\left\{\vartheta_{m}(x)\right\}_{n=1}^{\infty}$ in the space $L_{2}[0, l]$, implies $U(t, x)=0$ almost everywhere on $[0, l]$ for all $t \in[-p, q]$.

Since, by virtue of the first condition of the problem 1 , the function $U(t, x)$ is continuous in $\bar{\Omega}$. Therefore, the solution of the problem 1 is unique. The Theorem 1 was proved.

\section{Justification of the existence of a solution of the problem 1}

Substituting the expansions (9) and (10) into equation (1), we obtain

$$
\begin{gathered}
\sum_{n=1}^{\infty} \vartheta_{n}(x){ }_{c} D_{0 t}^{\alpha} u_{n}(t)=\sum_{n=1}^{\infty} f_{n}(t) \vartheta_{n}(x)-\sum_{n=1}^{\infty} \rho_{n}^{2} u_{n}(t) \vartheta_{n}(x),(t, x) \in \Omega_{1}, \\
\sum_{n=1}^{\infty} u_{n}^{\prime \prime}(t) \vartheta_{n}(x)=\sum_{n=1}^{\infty} f_{n}(t) \vartheta_{n}(x)-\sum_{n=1}^{\infty} \rho_{n}^{2} u_{n}(t) \vartheta_{n}(x),(t, x) \in \Omega_{2} .
\end{gathered}
$$


Hence, taking into account the fact that the system of eigenvalue functions $\left\{\vartheta_{n}(x)\right\}_{n=1}^{\infty}$ form a complete system of orthonormal functions in the space $L_{2}[0, l]$, we arrive at countable systems of differential equations

$$
\begin{gathered}
{ }_{c} D_{0 t}^{\alpha}\left[u_{n}(t)\right]+\rho_{n}^{2} u_{n}(t)=f_{n}(t), \quad 0 \leq t \leq q, \\
u_{n}^{\prime \prime}(t)+\rho_{n}^{2} u_{n}(t)=f_{n}(t), \quad-p \leq t \leq 0, n \in \mathbb{N} .
\end{gathered}
$$

By virtue of (3), the equation (19) takes the form

$$
I_{0 t}^{1-\alpha} u_{n}^{\prime}(t)+\rho_{n}^{2} u_{n}(t)=f_{n}(t), 0 \leq t \leq q .
$$

Applying the operator $I_{0 t}^{\alpha}[\cdot]$ to both sides of equation (21) and taking

$$
I_{0 t}^{\alpha} I_{0 t}^{1-\alpha} u_{n}^{\prime}(t)=I_{0 t}^{1} u_{n}^{\prime}(t)=u_{n}(t)-u_{n}(0)
$$

into account we bring this equation to the Volterra integral equation of the second kind with respect to the unknown function $u_{n}(t)$ :

$$
\begin{gathered}
u_{n}(t)+\frac{\rho_{n}^{2}}{\Gamma(\alpha)} \int_{0}^{t}(t-\tau)^{\alpha-1} u_{n}(\tau) d \tau= \\
=u_{n}(0)+\frac{1}{\Gamma(\alpha)} \int_{0}^{t}(t-\tau)^{\alpha-1} f_{n}(\tau) d \tau, \quad 0 \leq t \leq q .
\end{gathered}
$$

Taking

$$
\begin{gathered}
\frac{1}{\Gamma(\beta)} \int_{0}^{z} t^{\sigma-1} E_{\alpha, \sigma}\left(\lambda t^{\alpha}\right)(z-t)^{\beta-1} d t=z^{\sigma+\beta-1} E_{\alpha, \sigma+\beta}\left(\lambda z^{\alpha}\right), \\
\frac{1}{\Gamma(\sigma)}+z E_{\alpha, \alpha+\sigma}(z)=E_{\alpha, \sigma}(z), \sigma>0, \beta>0
\end{gathered}
$$

into account we obtain the solution of the equation (22) in the form

$$
\begin{gathered}
u_{n}(t)=c_{n} E_{\frac{1}{\alpha}}\left(-\rho_{n}^{2} t^{\alpha}, 1\right)+ \\
+\int_{0}^{t}(t-\tau)^{\alpha-1} E_{\frac{1}{\alpha}}\left(-\rho_{n}^{2}(\tau-t)^{\alpha}, \alpha\right) f_{n}(\tau) d \tau, 0 \leq t \leq q,
\end{gathered}
$$

where $c_{n}$ is arbitrary constant.

Solving the equation (20) by the Lagrange method, we obtain the representation

$$
u_{n}(t)=a_{n} \cos \rho_{n} t+b_{n} \sin \rho_{n} t+\frac{1}{\rho_{n}} \int_{t}^{0} f_{n}(\tau) \sin \rho_{n}(\tau-t) d \tau, \quad-p \leq t \leq 0,
$$

where $a_{n}, b_{n}$ are arbitrary constants.

Applying the gluing conditions (14)-(16) for $m=n$ to representations (23) and (24), we derive unknown coefficients $a_{n}, b_{n}, c_{n}$ :

$$
c_{n}=a_{n}=\frac{f_{n}(0)}{\rho_{n}^{2}}, b_{n}=0 .
$$

Substituting (25) into (23) and into (24), we obtain the following representations

$$
\begin{gathered}
u_{n}(t)=\frac{f_{n}(0)}{\rho_{n}^{2}} E_{\frac{1}{\alpha}}\left(-\rho_{n}^{2} t^{\alpha}, 1\right)+ \\
+\int_{0}^{t}(t-\tau)^{\alpha-1} E_{\frac{1}{\alpha}}\left(-\rho_{n}^{2}(\tau-t)^{\alpha}, \alpha\right) f_{n}(\tau) d \tau, 0 \leq t \leq q,
\end{gathered}
$$




$$
u_{n}(t)=\frac{f_{n}(0)}{\rho_{n}^{2}} \cos \rho_{n} t+\frac{1}{\rho_{n}} \int_{t}^{0} f_{n}(\tau) \sin \rho_{n}(\tau-t) d \tau,-p \leq t \leq 0 .
$$

Substituting (26) and (27) into the Fourier series (9), we formally represent the solution of the problem 1 in the form of the following Fourier series

$$
\begin{gathered}
U(t, x)=\sum_{n=1}^{\infty} \vartheta_{n}(x)\left[\frac{f_{n}(0)}{\rho_{n}^{2}} E_{\frac{1}{\alpha}}\left(-\rho_{n}^{2} t^{\alpha}, 1\right)+\right. \\
\left.+\int_{0}^{t}(t-\tau)^{\alpha-1} E_{\frac{1}{\alpha}}\left(-\rho_{n}^{2}(t-\tau)^{\alpha}, \alpha\right) f_{n}(\tau) d \tau\right], \quad(t, x) \in \Omega_{1}, \\
U(t, x)=\sum_{n=1}^{\infty} \vartheta_{n}(x)\left[\frac{f_{n}(0)}{\rho_{n}^{2}} \cos \rho_{n} t+\frac{1}{\rho_{n}} \int_{t}^{0} f_{n}(\tau) \sin \rho_{n}(\tau-t) d \tau\right],(t, x) \in \Omega_{2} .
\end{gathered}
$$

Theorem 2. Let the functions $f(t, x), f_{x}(t, x), f_{x x}(t, x), f_{t}(t, x)$ be continuous in $\bar{\Omega}$ and $f_{t t}(t, x) \in$ $\in L_{2}(\Omega), f(t, 0)=f(t, l)=0,-p \leq t \leq q$. Then a regular solution of the problem 1 exists and is defined in the form of series (28) and (29).

Proof. In proving the convergence of the series (28) and (29) with the properties of the problem 1, an important role are played the applications of the Cauchy-Schwartz inequality and Bessel inequality. First, estimate the following functions

$$
\begin{gathered}
\frac{1}{\rho_{n}^{2}}=\frac{1}{\mu_{n}^{2}+\lambda^{2}} \leq \frac{1}{\mu_{n}^{2}}=\left(\frac{l}{\pi}\right)^{2} \frac{1}{n^{2}}, \rho_{n}=\sqrt{\mu_{n}^{2}+\lambda^{2}}, \mu_{n}=\frac{\pi n}{l}, n \in \mathbb{N} ; \\
\left|E_{\frac{1}{\alpha}}\left(-\rho_{n}^{2}(t-\tau)^{\alpha}, \alpha\right)\right| \leq \frac{C}{1+\rho_{n}^{2}(t-\tau)^{\alpha}} \leq \frac{C}{\rho_{n}^{2}(t-\tau)^{\alpha}} \leq \frac{(t-\tau)^{1-\alpha} C}{\rho_{n}^{2}(t-\tau)} \leq \\
\leq \frac{(t-\tau)^{-\alpha} C}{\rho_{n}^{2}} \leq \frac{M_{0}}{\rho_{n}^{2}}, M_{0} \geq(t-\tau)^{-\alpha} C, 0<C=\mathrm{const} ; \\
\left|\int_{0}^{t}(t-\tau)^{\alpha-1} d \tau\right|=\left|\int_{0}^{t}(t-\tau)^{\alpha-1} d(t-\tau)\right|=\left|\frac{(t-\tau)^{\alpha}}{\alpha}\right|_{\tau=0}^{\tau=t} \leq \frac{q^{\alpha}}{\alpha} .
\end{gathered}
$$

Then applying the Cauchy-Schwartz inequality and Bessel inequality to the series (28), we obtain the following estimate

$$
\begin{aligned}
& |U(t, x)| \leq \sqrt{\frac{2}{l}} \sum_{n=1}^{\infty}\left[\left|\frac{f_{n}(0)}{\rho_{n}^{2}}\right| \cdot\left|E_{\frac{1}{\alpha}}\left(-\rho_{n}^{2} t^{\alpha}, 1\right)\right|+\right. \\
& \left.+\int_{0}^{t}(t-\tau)^{\alpha-1}\left|E_{\frac{1}{\alpha}}\left(-\rho_{n}^{2}(t-\tau)^{\alpha}, \alpha\right)\right| \cdot\left|f_{n}(\tau)\right| d \tau\right] \leq \\
& \leq \sqrt{\frac{2}{l}} \sum_{n=1}^{\infty} \frac{\left|f_{n}(0)\right|}{\rho_{n}^{2}} \cdot \frac{M_{0}}{\rho_{n}^{2}}+\sqrt{\frac{2}{l}} \sum_{n=1}^{\infty} M_{0} \frac{\max _{0 \leq t \leq q}\left|f_{n}(t)\right|}{\rho_{n}^{2}}\left|\int_{0}^{t}(t-\tau)^{\alpha-1} d \tau\right| \leq \\
& \leq M_{0} \sqrt{\frac{2}{l}} \sqrt{\sum_{n=1}^{\infty} \frac{1}{\mu_{n}^{8}}} \sqrt{\sum_{n=1}^{\infty}\left|\int_{0}^{l} f(0, x) \vartheta_{n}(x) d x\right|^{2}}+ \\
& +M_{0} \sqrt{\frac{2}{l}} \frac{q^{\alpha}}{\alpha} \sqrt{\sum_{n=1}^{\infty} \frac{1}{\mu_{n}^{4}}} \sqrt{\sum_{n=1}^{\infty} \max _{0 \leq t \leq q}\left|\int_{0}^{l} f(t, x) \vartheta_{n}(x) d x\right|^{2}} \leq
\end{aligned}
$$




$$
\leq 2 \sqrt{\frac{2}{l}} M_{0} M_{1} M_{2} M_{3} \max _{0 \leq t \leq q}\|f(t, x)\|_{L_{2}[0 ; l]}<\infty,(t, x) \in \Omega_{1},
$$

where

$$
M_{1}=\max \left\{\frac{q^{\alpha}}{\alpha} ; 1\right\} ; M_{2}=\max \left\{\left(\frac{l}{\pi}\right)^{2} ;\left(\frac{l}{\pi}\right)^{4}\right\} ; M_{3}=\sqrt{\sum_{n=1}^{\infty} \frac{1}{n^{4}}} .
$$

Similarly to estimate (30), for series (29) we obtain the estimate

$$
\begin{aligned}
& |U(t, x)| \leq \sqrt{\frac{2}{l}} \sum_{n=1}^{\infty} \frac{\left|f_{n}(0)\right|}{\rho_{n}^{2}}+p \sum_{n=1}^{\infty} M_{0} \frac{\max _{p \leq t \leq 0}\left|f_{n}(t)\right|}{\rho_{n}} \leq \\
& \leq \sqrt{\frac{2}{l}} \sqrt{\sum_{n=1}^{\infty} \frac{1}{\mu_{n}^{4}}} \sqrt{\sum_{n=1}^{\infty}\left|f_{n}(0)\right|^{2}}+p \sqrt{\frac{2}{l}} \sqrt{\sum_{n=1}^{\infty} \frac{1}{\mu_{n}^{2}}} \sqrt{\sum_{n=1}^{\infty} \max _{-p \leq t \leq 0}\left|f_{n}(t)\right|^{2}} \leq \\
& \leq 2 \sqrt{\frac{2}{l}} N_{0} N_{1} N_{2} \max _{-p \leq t \leq 0}\|f(t, x)\|_{L_{2}[0 ; l]}<\infty,(t, x) \in \Omega_{2},
\end{aligned}
$$

where

$$
N_{0}=\max \{p ; 1\} ; N_{1}=\max \left\{\frac{l}{\pi} ;\left(\frac{l}{\pi}\right)^{2}\right\} ; N_{2}=\sqrt{\sum_{n=1}^{\infty} \frac{1}{n^{2}}} .
$$

By virtue of the estimates (30) and (31), we conclude that the series (28) and (29) absolutely and uniformly converge.

Similarly to the case of series (28) and (29), it is easy to check, that the series

$$
\begin{gathered}
t^{2-\alpha} U_{t t}(t, x)=\sum_{n=1}^{\infty} t^{2-\alpha} u_{n}^{\prime \prime}(t) \vartheta_{n}(x),(t, x) \in \Omega_{1}, \\
U_{t t}(t, x)=\sum_{n=1}^{\infty} u_{n}^{\prime \prime}(t) \vartheta_{n}(x),(t, x) \in \Omega_{2} .
\end{gathered}
$$

are convergent.

Now we prove the convergence of the following series

$$
\begin{gathered}
U_{x x}(t, x)=-\sum_{n=1}^{\infty} \rho_{n}^{2} \vartheta_{n}(x)\left[\frac{f_{n}(0)}{\rho_{n}^{2}} E_{\frac{1}{\alpha}}\left(-\rho_{n}^{2} t^{\alpha}, 1\right)+\right. \\
\left.+\int_{0}^{t}(t-\tau)^{\alpha-1} E_{\frac{1}{\alpha}}\left(-\rho_{n}^{2}(t-\tau)^{\alpha}, \alpha\right) f_{n}(\tau) d \tau\right], \quad(t, x) \in \Omega_{1}, \\
U_{x x}(t, x)=-\sum_{n=1}^{\infty} \rho_{n}^{2} \vartheta_{n}(x) \times \\
\times\left[\frac{f_{n}(0)}{\rho_{n}^{2}} \cos \rho_{n} t+\frac{1}{\rho_{n}} \int_{t}^{0} f_{n}(\tau) \sin \rho_{n}(\tau-t) d \tau\right], \quad(t, x) \in \Omega_{2} .
\end{gathered}
$$

Integrating twice in parts the integral $f_{n}(t)=\int_{0}^{l} f(t, x) \vartheta_{n}(x) d x$ with respect to $x$, we obtain $f_{n}(t)=-\rho_{n}^{-2} f_{n}^{\prime \prime}(t)$, where $f_{n}^{\prime \prime}(t)=\int_{0}^{l} f_{x x}(t, x) \vartheta_{n}(x) d x$.

Then for the series (32) and (33), respectively, we derive the following estimates

$$
\left|U_{x x}(t, x)\right| \leq \sqrt{\frac{2}{l}} \sum_{n=1}^{\infty} \rho_{n}^{2}\left[\left|\frac{f_{n}(0)}{\rho_{n}^{2}}\right| \cdot\left|E_{\frac{1}{\alpha}}\left(-\rho_{n}^{2} t^{\alpha}, 1\right)\right|+\right.
$$




$$
\begin{gathered}
\left.+\int_{0}^{t}(t-\tau)^{\alpha-1}\left|E_{\frac{1}{\alpha}}\left(-\rho_{n}^{2}(t-\tau)^{\alpha}, \alpha\right)\right| \cdot\left|f_{n}(\tau)\right| d \tau\right] \leq \\
\leq \sqrt{\frac{2}{l}} \sum_{n=1}^{\infty} \frac{\left|f_{n}^{\prime \prime}(0)\right|}{\rho_{n}^{2}} \cdot \frac{M_{0}}{\rho_{n}^{2}}+\sum_{n=1}^{\infty} M_{0} \frac{\max _{0 \leq t \leq q}\left|f_{n}^{\prime \prime}(t)\right|}{\rho_{n}^{2}}\left|\int_{0}^{t}(t-\tau)^{\alpha-1} d \tau\right| \leq \\
\leq 2 \sqrt{\frac{2}{l}} M_{0} M_{1} M_{2} M_{3} \max _{0 \leq t \leq q}\left\|f_{x x}(t, x)\right\|_{L_{2}[0 ; l]}<\infty,(t, x) \in \Omega_{1}, \\
\left|U_{x x}(t, x)\right| \leq \sqrt{\frac{2}{l}} \sum_{n=1}^{\infty} \frac{\left|f_{n}^{\prime \prime}(0)\right|}{\rho_{n}^{2}}+p \sum_{n=1}^{\infty} M_{0} \frac{\max _{p \leq t \leq 0}\left|f_{n}^{\prime \prime}(t)\right|}{\rho_{n}} \leq \\
\leq 2 \sqrt{\frac{2}{l}} N_{0} N_{1} N_{2} \max _{-p \leq t \leq 0}\left\|f_{x x}(t, x)\right\|_{L_{2}[0 ; l]}<\infty,(t, x) \in \Omega_{2} .
\end{gathered}
$$

By virtue of the estimates (34) and (35), we conclude that the series (32) and (33) absolutely and uniformly converge. It follows that the series (28) and (29) satisfy all the properties of the problem 1. Theorem 2 is proved.

Using the same method, we can establish a unique solvability of the following problem.

Problem 2. Find a solution $U(t, x)$ of equation (1) that is regular in the domain $\Omega$ and satisfies all the conditions of problem 1 except (8), which is replaced by the following condition

$$
U_{x}(t, 0)=0, \quad U_{x}(t, 0)=0, \quad-p \leq t \leq q .
$$

\section{References}

1 Sabitov K.B. Dirichlet problem for mixed type equations in a rectangular domain / K.B. Sabitov // Doklady Mathematics. - 2007. - 75. - № 2. - P. 193-196.

2 Капустин Н.Ю. О спектральных задачах со спектральным параметром в граничном условии / Н.Ю. Капустин, Е.И. Моисеев // Дифференциальные уравнения. - 1997. - 33. - № 1. - С. 115-119.

3 Сабитов К.Б. Задача Трикоми для уравнения смешанного параболо-гиперболического в прямоугольной области / К.Б. Сабитов // Математические заметки. - 2009. - 86. - № 2. - С. 249-254.

4 Amanov D. On a Generalization of the Initial-Boundary Problem for the Vibrating String Equation / D. Amanov, G. Ibragimov, A. Kilicman // Symmetry. — 2019. — 11. — № 1. - ID 73.

5 Yuldashev T.K. On a boundary-value problem for Boussinesq type nonlinear integro-differential equation with reflecting argument / T.K. Yuldashev // Lobachevskii Journal of Mathematics. - 2020. - 41. № 1. - P. 111-123.

6 Пулькина Л.С. Задача с динамическим нелокальным условием для псевдогиперболического уравнения / Л.С. Пулькина // Изв. вузов. Математика. - 2016. - № 9. - С. 42-50.

7 Пулькина Л.С. Задача с нелокальным по времени условием для многомерного гиперболического уравнения / Л.С. Пулькина, А.Е. Савенкова // Изв. вузов. Математика. - 2016. - № 10. - С. 4152.

8 Сабитова Ю.К. Задача Дирихле для уравнения Лаврентьева-Бицадзе с нагруженными слагаемыми / Ю.К. Сабитова // Изв. вузов. Математика. - 2018. - № 9. - С. 42-58.

9 Юлдашев Т.К. О разрешимости краевой задачи для дифференциальных уравнений Буссинеска / Т.К. Юлдашев // Дифференц. уравнения. - 2018. - 54. - № 10. - С. 1411-1419.

10 Sidorov N.A. Classic solutions of boundary value problems for partial differential equations with operator of finite index in the main part of equation / N.A. Sidorov // The Bulletin of Irkutsk State University. Series Mathematics. - 2019. - 27. - P. 55-70.

11 Assanova A.T. A nonlocal problem for loaded partial differential equations of fourth order / A.T. Assanova, A.E. Imanchiyev, Z.M. Kadirbayeva // Bulletin of the Karaganda University-Mathematics. - 2020. 97. - № 1. - P. 6-16. 
12 Lundstrom B.N. Fractional differentiation by neocortical pyramidal neurons / B.N. Lundstrom, M.H. Higgs, W.J. Spain, A.L. Fairhall // Nature Neuroscience. - 2018. — 11. — № 11. — P. 1335-1342.

13 Vinagre B.M. Some approximations of fractional order operators used in control theory and application / B.M. Vinagre, I. Podlubny, A. Hernandez, V. Feliu // Fractional Calculus and its Applications. - 2000. - 3. - № 3. - P. 231-248.

14 Monje C.A. Fractional-order systems and controls: fundamentals and applications / C.A. Monje, Y.Q. Chen, B.M. Vinagre, D. Xue, V. Feliu-Batlle. - Springer, Science \& Business Media, 2010.

15 Gorenflo R. On the Cauchy and multipoint problems for partial pseudo-differential equations of fractional order / R. Gorenflo, Y.F. Luchko, S.R. Umarov // Fractional Calculus and its Applications. - 2000. 3. - P. 249-275.

16 Gorenflo R. Wright fractions as scale-invariant solutions of the diffusion-wave equation / R. Gorenflo, Y.F. Luchko, F. Mainardi // Journal of Computational and Applied Mathematics._- 2000. - 118. P. 175-191.

17 Karimov E.T. A boundary problem with integral gluing condition for a parabolic-hyperbolic equation involving the Caputo fractional derivative / E.T. Karimov, J.S. Akhatov // Electronic Journal of Differential Equations. - 2014. - № 14. - ID 1. - P. 1-6.

18 Kilbas A.A. Cauchy problem for differential equation with Caputo derivative / A.A. Kilbas, S.A. Marzan // Fractional Calculus and its Applications. - 2004. - 7. - № 3. - P. 297-321.

19 Turmetov B. On Fractional Analogs of Dirichlet and Neumann Problems for the Laplace Equation / B. Turmetov, K. Nazarova // Mediterranean Journal of Mathematics. — 2019. — 16. — № 59.

20 Yuldashev T.K. Boundary value problem for weak nonlinear partial differential equations of mixed type with fractional Hilfer operator / T.K. Yuldashev, B.J. Kadirkulov // Axioms. - 2020. - 9. — № 2. ID $68 .-$ P. 1-19.

21 Yuldashev T.K. Nonlocal problem for a mixed type fourth-order differential equation with Hilfer fractional operator / T.K. Yuldashev, B.J. Kadirkulov // Ural Mathematical Journal. — 2020. — 6. — № 1. — P. 153167.

22 Yuldashev T.K. Inverse problem for a mixed type integro-differential equation with fractional order Caputo operators and spectral parameters / T.K. Yuldashev, E.T. Karimov // Axioms. - 2020. - 9. 4. - ID 121. - P. 1-19.

23 Islomov B.I. On a boundary value problem for a parabolic-hyperbolic equation with fractional order Caputo operator in rectangular domain / B.I. Islomov, U.Sh. Ubaydullayev // Lobachevskii Journal of Mathematics. - 2020. - 41. - № 9. - P. 1801-1810.

Т.К. Юлдашев, Б.И. Исломов, У.Ш. Убайдуллаев

\title{
Капуто операторы бар аралас типті бөлшек дифференциалдық теңдеуге арналған шеттік есептер туралы
}

\begin{abstract}
Мақалада тікбұрышты аймақтағы бөлшек Капуто операторы бар параболалық гиперболалық типтес біртекті емес аралас дифференциалдық теңдеу үшін кеңістіктік айнымалыға қатысты бірінші және екінші типтегі шеттік есептер зерттелді. Мұндай шеттік есептерді зерттеу кезінде бірінші аргумент бойынша шекаралық шарт қоюдан бас тартылды және оның орнына қосымша үзіліссіздік шарты қолданылды. Бұл ретте біржақты шешілуін негіздеу үшін шекаралық облысқа арналған шарттар алынып тасталады. Бұл мақала авторлары қарастырған шеттік есептердің біржақты шешілу критерийін әлсіретуге мүмкіндік береді. Шешім біртекті спектрлік есептерге сәйкес келетін өз функциялары бар Фурье қатарлары түрінде жасалды. Фурье қатарларының конвергенциясы осы аралас теңдеудің тұрақты шешімі ретінде алынды.
\end{abstract}

Kiлm сөздер: аралас дифференциалдық теңдеу, бөлшек реті, Капуто операторы, модельдік емес теңдеу, Фурье қатары, үзіліссіздік шарттары, біржақты шешілу. 
Т.К. Юлдашев, Б.И. Исломов, У.Ш. Убайдуллаев

\title{
О краевых задачах для дробного дифференциального уравнения смешанного типа с оператором Капуто
}

\begin{abstract}
В статье исследованы краевые задачи первого и второго родов относительно пространственного переменного для смешанного неоднородного дифференциального уравнения параболо-гиперболического типа с дробным оператором Капуто в прямоугольной области. При исследовании таких краевых задач авторы отказались от задания граничного условия по первому аргументу и использовали вместо этого дополнительное условие склеивания. При этом в обосновании однозначной разрешимости снимаются условия на граничную область. Это позволило авторам статьи ослабить критерий однозначной разрешимости рассматриваемых краевых задач. Решение построено в виде рядов Фурье с собственными функциями, соответствующими однородным спектральным задачам. Получены оценки сходимости рядов Фурье как регулярное решение этого смешанного уравнения.
\end{abstract}

Ключевые слова: смешанное дифференциальное уравнение, дробный порядок, оператор Капуто, немодельное уравнение, ряд Фурье, условия склеивания, однозначная разрешимость.

\section{References}

1 Sabitov, K.B. (2007). Dirichlet problem for mixed type equations in a rectangular domain. Doklady Mathematics, 75(2), 193-196.

2 Kapustin, N.Yu., \& Moiseev, E.I. (1997). O spektralnykh zadachakh so spektralnym parametrom v hranichnom uslovii [On spectral problems with a spectral parameter in the boundary condition]. Differentsialnye uravneniia - Differential Equations, 33(1), 116-120 [in Russian].

3 Sabitov, K.B. (2009). Zadacha Trikomi dlia uravneniia smeshannoho parabolo-hiperbolicheskoho v priamouholnoi oblasti [The Tricomi problem for a mixed parabolic-hyperbolic equation in a rectangular domain]. Matematicheskie zametki - Mathematical Notes, 86(2), 249-254 [in Russian].

4 Amanov, D., Ibragimov, G., \& Kilicman, A. (2019). On a Generalization of the Initial-Boundary Problem for the Vibrating String Equation. Symmetry, 11(1), ID 73.

5 Yuldashev, T.K. (2020). On a boundary-value problem for Boussinesq type nonlinear integro-differential equation with reflecting argument. Lobachevskii Journal of Mathematics, 41(1), 111-123.

6 Pul'kina, L.S. (2016). Zadacha s dinamicheskim nelokalnym usloviem dlia psevdohiperbolicheskoho uravneniia [A problem with dynamic nonlocal condition for pseudohyperbolic equation]. Izvestiia vuzov. Matematika - Russian Mathtmatics (Iz. VUZ), 60(9), 38-45 [in Russian].

7 Pul'kina, L.S., \& Savenkova, A.E. (2016). Zadacha s nelokalnym po vremeni usloviem dlia mnohomernoho hiperbolicheskoho uravneniia [A problem with a nonlocal with respect to time condition for multidimensional hyperbolic equations]. Izvestiia vuzov. Matematika - Russian Mathematics (Iz. VUZ), 60(10), 33-43 [in Russian].

8 Sabitova, Yu.K. (2018). Zadacha Dirikhle dlia uravneniia Lavrenteva-Bitsadze s nahruzhennymi slahaemymi [Dirichlet problem for Lavrent'ev-Bitsadze equation with loaded summands]. Izvestiia vuzov. Matematika - Russian Mathematics (Iz. VUZ), 62(9), 35-51 [in Russian].

9 Yuldashev, T.K. (2018). O razreshimosti kraevoi zadachi dlia differentsialnykh uravnenii Bussineska [Solvability of a boundary value problem for a differential equation of the Boussinesq type]. Differentsialnye uravneniia - Differerential Equations, 54(10), 1384-1393 [in Russian].

10 Sidorov, N.A. (2019). Classic solutions of boundary value problems for partial differential equations with operator of finite index in the main part of equation. The Bulletin of Irkutsk State University. Series Mathematics, 27, 55-70.

11 Assanova, A.T., Imanchiyev, A.E., \& Kadirbayeva, Z.M. (2020). A nonlocal problem for loaded partial differential equations of fourth order. Bulletin of the Karaganda university-Mathematics, 97(1), 6-16.

12 Lundstrom, B.N., Higgs, M.H., Spain, W.J., \& Fairhall, A.L. (2018). Fractional differentiation by neocortical pyramidal neurons. Nature Neuroscience, 11(11), 1335-1342. 
13 Vinagre, B.M., Podlubny, I., Hernandez, A., \& Feliu, V. (2000). Some approximations of fractional order operators used in control theory and application. Fractional Calculus and its Applications, 3(3), $231-248$.

14 Monje, C.A., Chen, Y.Q., Vinagre, B.M., Xue, D., \& Feliu-Batlle, V. (2010). Fractional-order systems and controls: fundamentals and applications. Springer, Science \& Business Media, 2010.

15 Gorenflo, R., Luchko, Y.F., \& Umarov, S.R. (2000). On the Cauchy and multipoint problems for partial pseudo-differential equations of fractional order. Fractional Calculus and its Applications, 3, 249-275.

16 Gorenflo, R., Luchko, Y.F., \& Mainardi F. (2000). Wright fractions as scale-invariant solutions of the diffusion-wave equation. Journal of Computational and Applied Mathematics, 118, 175-191.

17 Karimov, E.T., \& Akhatov, J.S. (2014). A boundary problem with integral gluing condition for a parabolichyperbolic equation involving the Caputo fractional derivative. Electronic Journal of Differential Equations, $14(1), 1-6$.

18 Kilbas, A.A., \& Marzan, S.A. (2004). Cauchy problem for differential equation with Caputo derivative. Fractional Calculus and its Applications, 7(3), 297-321.

19 Turmetov, B., \& Nazarova, K. (2019). On Fractional Analogs of Dirichlet and Neumann Problems for the Laplace Equation. Mediterranean Journal of Mathematics, 16(3), 1-17.

20 Yuldashev, T.K., \& Kadirkulov, B.J. (2020). Boundary value problem for weak nonlinear partial differential equations of mixed type with fractional Hilfer operator. Axioms, 9, 2(68), 1-19.

21 Yuldashev, T.K., \& Kadirkulov, B.J. (2020). Nonlocal problem for a mixed type fourth-order differential equation with Hilfer fractional operator. Ural Mathematics Journal, 6(1), 153-167.

22 Yuldashev, T.K., \& Karimov, E.T. (2020). Inverse problem for a mixed type integro-differential equation with fractional order Caputo operators and spectral parameters. Axioms, 9, 4(121), 1-19.

23 Islomov, B.I., \& Ubaydullayev, U.Sh. (2020). On a boundary value problem for a parabolic-hyperbolic equation with fractional order Caputo operator in rectangular domain. Lobachevskii Journal of Mathematics, 41(9), 1801-1810. 


\author{
T.K. Yuldashev ${ }^{1, *}$, S.K. Zarifzoda ${ }^{2}$ \\ ${ }^{1}$ National University of Uzbekistan, Tashkent, Uzbekistan; \\ ${ }^{2}$ Tajik National University, Dushanbe, Tajikistan \\ (E-mail: tursun.k.yuldashev@gmail.com, sarvar8383@list.ru)
}

\title{
On a New Class of Singular Integro-differential Equations
}

\begin{abstract}
In this paper for a new class of model and non-model partial integro-differential equations with singularity in the kernel, we obtained integral representation of family of solutions by aid of arbitrary functions. Such type of integro-differential equations are different from Cauchy-type singular integro-differential equations. Cauchy-type singular integro-differential equations are studied by the methods of the theory of analytic functions. In the process of our research the new types of singular integro-differential operators are introduced and main property of entered operators are learned. It is shown that the solution of studied equation is equivalent to the solution of system of two equations with respect to $x$ and $y$, one of which is integral equation and the other is integro-differential equation. Further, non-model integro-differential equations are studied by regularization method. This regularization method for non-model equation is based on selecting and analysis of a model part of the equation and reduced to the solution of two second kind Volterra type integral equations with weak singularity in the kernel. It is shown that the presence of a non-model part in the equation does not affect to the general structure of the solutions. From here investigation of the model equations for given class of the integro-differential equations becomes important. In the cases, when the solution of given integro-differential equation depends on any arbitrary functions, a Cauchy type problems are investigated.
\end{abstract}

Keywords: singular integro-differential equation, model equation, non model equation, characteristic equation, Cauchy type problem.

\section{Introduction}

In addition to the theory of differential and integral equations, the theory of integro-differential (I-D) equations with regular and singular coefficients plays an important role in theoretical and applied research. There are many scientific publications where theoretical or applied aspects of the theory of I-D equations are studied. Of particular interest is research on I-D equations with singular kernels. During the last years the theory of the I-D equations basically developed in two directions. The first direction is connected with the study of approximate solutions of I-D equations [1-10]. The second direction is connected with construction of the general theory for a new classes of the I-D equations [11-18]. Study of the various aspects of I-D equations in Banach spaces also concerns to the second direction [19-21]. Also, in last years the methods of solving the direct and inverse problems and problems with small parameters for the I-D equations [22-24] were actively developed.

One of section in the theory of the I-D equations, which is not studied completely, is the section of I-D equations with singular and super singular coefficients. Some results in this direction are received by integral transform methods in [25-27]. But, we note that the singularity in dependent of the studied problems has different nature. Therefore, the approach of separate authors to study the singular problems happens in different ways.

In the classical singular integro-differential equations the integrals basically we understood in sense of a principal value of Cauchy. Consequently, in solving some equations the methods of analytic functions are used. Unlike this, we will investigate such singular I-D equations, in which integrals are understood in ordinary sense of Riemann. Therefore, our approaches in studying the given problem are also different from the works [13-15]. This work is a further continuation of our research in [28, 29].

\footnotetext{
${ }^{*}$ Corresponding author.

E-mail: tursun.k.yuldashev@gmail.com
} 


\section{Formulation of problem and basic designation}

We denote: $D=\{(x, y): a<x<c, b<y<d\}$ and $\Gamma_{1}=\{a<x<c, y=b\}, \Gamma_{2}=\{x=a, b<y<d\}$. In the domain $D$ we consider a partial integro-differential equation with singular coefficients:

$$
\begin{aligned}
& \mathbf{K} \varphi \equiv \varphi_{x}^{\prime}(x, y)+\frac{A(x)}{x-a} \varphi+\int_{a}^{x} \frac{B(t)}{(t-a)^{2}} \varphi(t, y) d t+\int_{b}^{y} \frac{E(s)}{s-b} \varphi_{x}^{\prime}(x, s) d s+ \\
& +\int_{b}^{y} \frac{G(x, s)}{(x-a)(s-b)} \varphi(x, s) d s+\int_{a}^{x} \frac{d t}{(t-a)^{2}} \int_{b}^{y} \frac{H(t, s)}{s-b} \varphi(t, s) d s=f(x, y),
\end{aligned}
$$

where $A(x), B(x), E(y), G(x, y), H(x, y)$ are given functions connecting to each other by equalities $G(x, y)=A(x) E(y), H(x, y)=B(x) E(y) ; f(x, y)$ is the given function on the domain $D ; \varphi(x, y)$ is a unknown function.

An importance of studying equation (1) consists in the following idea:

1 The degree of singularity in the kernel of the studied equation with respect to $x$ is equal to 2 and with respect to $y$ is equal to 1 . Therefore the kernels of this equation are not Fredholm type kernels;

2 Singularity in this equation is not understood in the sense of a principal value of Cauchy;

3 For the solution of this equation we do not use methods of the theory of analytic functions;

4 We obtain the solution of the considering equation in the sense of generalized solution from the theory of regular I-D equations.

To solve the equation (1) we introduce some designations:

1 Through $\mathbb{C}_{x}^{\delta_{1}, \delta_{2}}(\bar{D})$ we denote a class of such functions, which have the first order continuous derivative with respect to the variable $x$ with an asymptotic behaviour

$$
f(x, y)=o\left[(x-a)^{\gamma_{1}},(y-b)^{\gamma_{2}}\right], \gamma_{1}>\delta_{1}, \gamma_{2}>\delta_{2} .
$$

2 We denote $\mathbb{C}^{\delta_{1}, 0}(\bar{D})=\mathbb{C}^{\delta_{1}}\left(\overline{\Gamma_{1}}\right)$ and $\mathbb{C}^{0, \delta_{2}}(\bar{D})=\mathbb{C}^{\delta_{2}}\left(\overline{\Gamma_{2}}\right)$.

3 Through $\mathbb{C}_{f(a)}^{\delta_{1}}\left(\bar{\Gamma}_{1}\right)$ we designate a class of such functions, for difference of which there is true the following $f(x)-f(a) \rightarrow 0$ as $x \rightarrow a$. This type of functions has an asymptotic behaviour

$$
f(x)-f(a)=o\left[(x-a)^{\gamma_{1}}\right], \gamma_{1}>\delta_{1} .
$$

If $f(a)=0$, then we have denotation $\mathbb{C}_{0}^{\delta_{1}}\left(\bar{\Gamma}_{1}\right)=\mathbb{C}^{\delta_{1}}\left(\overline{\Gamma_{1}}\right)$.

The solution of equation (1) we seek in the class $\mathbb{C}_{x}^{1,0}(\bar{D})$. For this purpose by $\Pi_{a, A(x), B(x)}^{x, 1,2}$ and $\Pi_{b, E(y)}^{y, 1}$ we designate operators acting on function $\varphi(x, y)$ by rules

$$
\begin{gathered}
\Pi_{a, A(x), B(x)}^{x, 1,2} \varphi(x, y) \equiv \varphi_{x}^{\prime}(x, y)+\frac{A(x)}{x-a} \varphi(x, y)+\int_{a}^{x} \frac{B(t)}{(t-a)^{2}} \varphi(t, y) d t \\
\Pi_{b, E(y)}^{y, 1} \varphi(x, y) \equiv \varphi(x, y)+\int_{b}^{y} \frac{E(s)}{s-b} \varphi(x, s) d s .
\end{gathered}
$$

If $A(x)=A=$ const, $B(x)=B=$ const and $E(x)=E=$ const, then operators $\Pi_{a, A, B}^{x, 1,2} \varphi(x, y)$ in (3) and $\Pi_{b, E}^{y, 1} \varphi(x, y)$ in (4) we call model operators and the equations corresponding to these operators we call model equations.

So, equation (1) by means of just entered operators we represent as

$$
\Pi_{a, A(x), B(x)}^{x, 1,2} \Pi_{b, E(y)}^{y, 1} \varphi(x, y)=f(x, y) .
$$

Then the solution of operator equation (5) is equivalent to the solution of the following system of I-D equations

$$
\left\{\begin{array}{l}
\Pi_{b, E(y)}^{y, 1} \varphi(x, y)=\psi(x, y) \\
\Pi_{a, A(x), B(x)}^{x, 1,2} \psi(x, y)=f(x, y),
\end{array}\right.
$$

where $\psi(x, y)$ is a new unknown function and the function $f(x, y)$ has an asymptotic behaviour (2). 
By separating model part of system (6) we obtain

$$
\left\{\begin{array}{l}
\Pi_{b, E(b)}^{y, 1} \varphi(x, y)=\Psi(x, y) \\
\Pi_{a, A(a), B(a)}^{x, 1,2} \psi(x, y)=F(x, y)
\end{array}\right.
$$

where

$$
\begin{gathered}
\Psi(x, y)=\psi(x, y)-\int_{b}^{y} \frac{E(s)-E(b)}{s-b} \varphi(x, s) d s, \\
F(x, y)=f(x, y)-\frac{A(x)-A(a)}{x-a} \psi(x, y)-\int_{a}^{x} \frac{B(t)-B(a)}{(t-a)^{2}} \psi(t, y) d t .
\end{gathered}
$$

It is obvious that the homogeneous equations of system (7) correspond to characteristic equations

$$
\begin{gathered}
1+\frac{E(b)}{\lambda}=0, \\
\mu+A(a)+\frac{B(a)}{\mu-1}=0 .
\end{gathered}
$$

In dependent of the roots of the characteristic equations (10), (11) we obtain the solution of the system of equations (7) in following form.

Let $\lambda=-E(b)>0$ and the roots of the characteristic equations (11) be real and different, $1<\mu_{1}<\mu_{2}$. Then the solution of nonhomogeneous equations (7) gives by formula:

$$
\left\{\begin{array}{l}
\varphi(x, y)=(y-b)^{\lambda} c_{1}(x)+\Psi(x, y)+\left(\Pi_{b, \lambda}^{y, \lambda+1}\right)^{-1} \Psi(x, y) \equiv E_{1} \\
\psi(x, y)=(x-a)^{\mu_{1}} c_{2}(y)+(x-a)^{\mu_{2}} c_{3}(y)+\left(\Pi_{a, 1-\mu_{1}, 1-\mu_{2}}^{x, \mu_{1}, \mu_{2}}\right)^{-1} F(x, y) \equiv E_{2}
\end{array}\right.
$$

where $E_{1}=E_{1}\left[c_{1}(x), \Psi(x, y)\right], E_{2}=E_{2}\left[c_{2}(y), c_{3}(y), F(x, y)\right]$, operators $\left(\Pi_{b, \lambda}^{y, \lambda+1}\right)^{-1}$ and $\left(\Pi_{a, 1-\mu_{1}, 1-\mu_{2}}^{x, \mu_{1}, \mu_{2}}\right)^{-1}$ are inverse to operators $\Pi_{b, E(b)}^{y, 1}$ and $\Pi_{a, A(a), B(a)}^{x, 1,2}$, respectively, and the explicit form of these operators are

$$
\begin{gathered}
\left(\Pi_{b, \lambda}^{y, \lambda+1}\right)^{-1} \Psi(x, y)=\lambda \int_{b}^{y}\left(\frac{y-b}{s-b}\right)^{\lambda} \frac{\Psi(x, y)}{s-b} d s, \\
\left(\Pi_{a, 1-\mu_{1}, 1-\mu_{2}}^{x, \mu_{1}, \mu_{2}}\right)^{-1} F(x, y)= \\
=\frac{1}{\sqrt{D_{1}}} \int_{a}^{x}\left[\left(1-\mu_{1}\right)\left(\frac{x-a}{t-a}\right)^{\mu_{1}}-\left(1-\mu_{2}\right)\left(\frac{x-a}{t-a}\right)^{\mu_{2}}\right] F(t, y) d t .
\end{gathered}
$$

Substituting the value of $\Psi(x, y)$ and $F(x, y)$ from (8), (9) into (12), after some transformations we come to solving the following Volterra type integral equations

$$
\left\{\begin{array}{l}
\varphi(x, y)+\int_{b}^{y} K_{1}(y, s) \varphi(x, s) d s=E_{1}\left[c_{1}(x), \psi(x, y)\right] \\
\psi(x, y)+\frac{1}{\sqrt{D_{1}}} \int_{a}^{x} K_{2}(x, t) \psi(x, y) d t=E_{2}\left[c_{2}(y), c_{3}(y), f(x, y)\right]
\end{array}\right.
$$

where

$$
\left\{\begin{array}{l}
K_{1}(y, s)=\left(\frac{y-b}{s-b}\right)^{\lambda} \frac{E(s)-E(b)}{s-b} \\
K_{2}(x, t)=\left[\left(1-\mu_{1}\right)\left(\frac{x-a}{t-a}\right)^{\mu_{1}}-\left(1-\mu_{2}\right)\left(\frac{x-a}{t-a}\right)^{\mu_{2}}\right] \frac{A(t)-A(a)}{t-a}+ \\
+\left[\left(\frac{x-a}{t-a}\right)^{\mu_{1}}-\left(\frac{x-a}{t-a}\right)^{\mu_{2}}\right] \frac{B(t)-B(a)}{t-a}
\end{array}\right.
$$


If we introduce new unknown functions as $\varphi_{1}(x, y)=\frac{\varphi(x, y)}{(y-b)^{\lambda}}, \psi_{1}(x, y)=\frac{\psi(x, y)}{(x-a)^{\mu_{1}}}$, then instead the integral equations (13) we solve the following integral equations

$$
\left\{\begin{array}{l}
\varphi_{1}(x, y)+\int_{b}^{y} K_{1,1}(y, s) \varphi_{1}(x, s) d s=\frac{E_{1}\left[c_{1}(x), \psi(x, y)\right]}{(y-b)^{\lambda}} \\
\psi_{1}(x, y)+\frac{1}{\sqrt{D_{1}}} \int_{a}^{x} K_{2,1}(x, t) \psi_{1}(x, y) d t=\frac{E_{2}\left[c_{2}(y), c_{3}(y), f(x, y)\right]}{(x-a)^{\mu_{1}}}
\end{array}\right.
$$

where

$$
\left\{\begin{array}{l}
K_{1,1}(y, s)=\frac{E(s)-E(b)}{s-b} \\
K_{2,1}(x, t)=\left[1-\mu_{1}-\left(1-\mu_{2}\right)\left(\frac{x-a}{t-a}\right)^{\mu_{2}-\mu_{1}}\right] \frac{A(t)-A(a)}{t-a}+ \\
+\left[1-\left(\frac{x-a}{t-a}\right)^{\mu_{2}-\mu_{1}}\right] \frac{B(t)-B(a)}{t-a}
\end{array}\right.
$$

If the following conditions are fulfilled

$$
\begin{aligned}
& A(x) \in \mathbb{C}_{A(a)}^{\mu_{2}-\mu_{1}}\left(\overline{\Gamma_{1}}\right), B(x) \in \mathbb{C}_{B(a)}^{\mu_{2}-\mu_{1}}\left(\overline{\Gamma_{1}}\right), E(y) \in \mathbb{C}_{E(b)}^{\varepsilon}\left(\overline{\Gamma_{2}}\right), \\
& f(x, y) \in \mathbb{C}^{\mu_{2}-1, \lambda-1}(\bar{D}),\left\{c_{2}(y), c_{3}(y)\right\} \in \mathbb{C}^{\lambda-1}\left(\overline{\Gamma_{2}}\right),
\end{aligned}
$$

then the integral equations (14) become a Volterra type integral equations with weak singularity in the kernel and with the continuous right-hand side function. The solution of equations (14) by means of resolvent we write as

$$
\left\{\begin{array}{l}
\varphi_{1}(x, y)=\frac{E_{1}\left[c_{1}(x), \psi(x, y)\right]}{(y-b)^{\lambda}}-\int_{b}^{y} \Gamma_{1}(y, s) \frac{E_{1}\left[c_{1}(x), \psi(x, s)\right]}{(s-b)^{\lambda}} d s \\
\psi_{1}(x, y)=\frac{E_{2}\left[c_{2}(y), c_{3}(y), f(x, y)\right]}{(x-a)^{\mu_{1}}}-\frac{1}{\sqrt{D_{1}}} \int_{a}^{x} \Gamma_{2}(x, t) \frac{E_{2}\left[c_{2}(y), c_{3}(y), f(t, y)\right]}{(t-a)^{\mu_{1}}} d t,
\end{array}\right.
$$

where $\Gamma_{1}(y, s), \Gamma_{2}(x, t)$ are corresponding resolvent of integral equations (14).

Now coming back to our unknown functions $\varphi(x, y), \psi(x, y)$ we find the solution of equation (5) (or equivalent equation (1)) as follow:

$$
\left\{\begin{array}{l}
\varphi(x, y)=E_{1}\left[c_{1}(x), \psi(x, y)\right]-\int_{b}^{y} \Gamma_{1}(y, s)\left(\frac{y-b}{s-b}\right)^{\lambda} E_{1}\left[c_{1}(x), \psi(x, s)\right] d s \\
\psi(x, y)=E_{2}\left[c_{2}(y), c_{3}(y), f(x, y)\right]- \\
-\frac{1}{\sqrt{D_{1}}} \int_{a}^{x} \Gamma_{2}(x, t)\left(\frac{x-a}{t-a}\right)^{\mu_{1}} E_{2}\left[c_{2}(y), c_{3}(y), f(t, y)\right] d t
\end{array}\right.
$$

So we proved:

Theorem 1. We assume that conditions (15) are fulfilled; $G(x, y)=A(x) E(y), H(x, y)=B(x) E(y)$ in I$\mathrm{D}$ equation (1) and the roots of the characteristic equations (10) and (11) are such that $\lambda=-E(b)>0$, $1<\mu_{1}<\mu_{2}$. Then the solution of equation (1) in the class $C_{x}^{1,0}(\bar{D})$ is presented by formula (16).

From the validity of this Theorem 1, the following corollary holds.

Corollary. We assume that conditions (15) are fulfilled; $G(x, y)=A(x) E(y), H(x, y)=B(x) E(y)$ in the I-D equation (1) and roots of the characteristic equations (10) and (11) are such that $\lambda=-E(b)<0, \mu_{1}<\mu_{2}<1$. Then the solution of equation (1) in the class $C_{x}^{1,0}(\bar{D})$ is presented by following formula

$$
\varphi(x, y)=E_{1}[0, \psi(x, y)]-\int_{b}^{y} \Gamma_{1}(y, s)\left(\frac{y-b}{s-b}\right)^{\lambda} E_{1}[0, \psi(x, s)] d s,
$$


where

$$
\psi(x, y)=E_{2}[0,0, f(x, y)]-\frac{1}{\sqrt{D_{1}}} \int_{a}^{x} \Gamma_{2}(x, t)\left(\frac{x-a}{t-a}\right)^{\mu_{1}} E_{2}[0,0, f(t, y)] d t .
$$

Let $\lambda=-E(b)>0$ and the roots of the characteristic equation (11) be real and equal, $\mu_{1}=\mu_{2}=\mu>1$. Then the solution to the second equation of system (7) we represent as:

where

$$
\begin{gathered}
\psi(x, y)=(x-a)^{\mu} c_{4}(y)+(x-a)^{\mu} \ln (x-a) c_{5}(y)+ \\
+\left(\Pi_{a, \mu-1,1}^{x, \mu}\right)^{-1} F(x, y) \equiv E_{3}\left[c_{4}(y), c_{5}(y), F(x, y)\right],
\end{gathered}
$$

$$
\left(\Pi_{a, \mu-1,1}^{x, \mu}\right)^{-1} F(x, y)=\int_{a}^{x}\left(\frac{x-a}{t-a}\right)^{\mu}\left[(\mu-1) \ln \left(\frac{x-a}{t-a}\right)+1\right] F(t, y) d t .
$$

Here the operator $\left(\Pi_{a, \mu-1,1}^{x, \mu}\right)^{-1}$ is inverse to I-D operator $\Pi_{a, A(a), B(a)}^{x, 1,2}$, when the roots of the characteristic equation (11) are real and equal.

In this case repeating the above-stated scheme without stopping in details we obtain the general solution of equation (11) in a following form:

$$
\varphi(x, y)=E_{1}\left[c_{1}(x), \psi(x, y)\right]-\int_{b}^{y} \Gamma_{1}(y, s)\left(\frac{y-b}{s-b}\right)^{\lambda} E_{1}\left[c_{1}(x), \psi(x, s)\right] d s,
$$

where

$$
\psi(x, y)=E_{3}\left[c_{4}(y), c_{5}(y), f(x, y)\right]-\int_{a}^{x} \Gamma_{3}(x, t)\left(\frac{x-a}{t-a}\right)^{\mu} E_{3}\left[c_{4}(y), c_{5}(y), f(t, y)\right] d t
$$

and $\Gamma_{3}(x, t)$ is the resolvent of the integral equation which is written in an explicit form.

Thus the following theorem takes place:

Theorem 2. We assume that the following conditions are fulfilled:

1) $G(x, y)=A(x) E(y), H(x, y)=B(x) E(y)$ in an I-D equation (1);

2) The roots of the characteristic equations (10) and (11) are such that $\lambda=-E(b)>0, \mu>1$;

3) The following inclusions take place

$$
\begin{aligned}
& A(x) \in \mathbb{C}_{A(a)}^{\varepsilon}\left(\overline{\Gamma_{1}}\right), B(x) \in \mathbb{C}_{B(a)}^{\varepsilon}\left(\overline{\Gamma_{1}}\right), E(y) \in \mathbb{C}_{E(b)}^{\varepsilon}\left(\overline{\Gamma_{2}}\right), \\
& f(x, y) \in \mathbb{C}^{\mu-1, \lambda-1}(\bar{D}),\left\{c_{4}(y), c_{5}(y)\right\} \in \mathbb{C}^{\lambda-1}\left(\overline{\Gamma_{2}}\right) .
\end{aligned}
$$

Then the solution of equation (1) in the class $C_{x}^{1,0}(\bar{D})$ can be represented by formula (17).

Remark 1. In this case, if $\lambda=-E(b)<0, \mu<1$, we obtain the unique solution of equation (1) in the form

$$
\varphi(x, y)=E_{1}[0, \psi(x, y)]-\int_{b}^{y} \Gamma_{1}(y, s)\left(\frac{y-b}{s-b}\right)^{\lambda} E_{1}[0, \psi(x, s)] d s,
$$

where

$$
\psi(x, y)=E_{3}[0,0, f(x, y)]-\int_{a}^{x} \Gamma_{3}(x, t)\left(\frac{x-a}{t-a}\right)^{\mu} E_{3}[0,0, f(t, y)] d t .
$$

Let be $\lambda=-E(b)>0$ and the roots of the characteristic equation (11) are complex, conjugate and

$$
\mu_{1,2}=\frac{1-A(a)}{2} \pm \frac{\sqrt{4 B(a)-(1+A(a))^{2}}}{2} i \equiv \alpha \pm \beta i
$$

Then we can write the solution to the second equation of system (7) as:

$$
\begin{aligned}
\psi(x, y)= & (x-a)^{\alpha}\left\{\cos [\beta \ln (x-a)] c_{6}(y)+\sin [\beta \ln (x-a)] c_{7}(y)\right\}+ \\
& +\left(\Pi_{a, \alpha-1, \beta}^{x, \alpha}\right)^{-1} F(x, y) \equiv E_{4}\left[c_{6}(y), c_{7}(y), F(x, y)\right],
\end{aligned}
$$


where

$$
\begin{gathered}
\left(\Pi_{a, \alpha-1, \beta}^{x, \alpha}\right)^{-1} F(x, y)=\frac{1}{\beta} \int_{a}^{x}\left(\frac{x-a}{t-a}\right)^{\alpha}\left\{(\alpha-1) \sin \left[\beta \ln \left(\frac{x-a}{t-a}\right)\right]+\right. \\
\left.+\beta \cos \left[\beta \ln \left(\frac{x-a}{t-a}\right)\right]\right\} f(x, s) d t
\end{gathered}
$$

Here the operator $\left(\Pi_{a, \alpha-1, \beta}^{x, \alpha}\right)^{-1}$ is inverse to I-D operator $\Pi_{a, A(a), B(a)}^{x, 1,2}$, when the roots of the characteristic equation (11) are complex and conjugate.

In this case too repeating the above-stated scheme by means of resolvent of corresponding Volterra type integral equation we obtain the general solution of equation (1) in a following form:

$$
\varphi(x, y)=E_{1}\left[c_{1}(x), \psi(x, y)\right]-\int_{b}^{y} \Gamma_{1}(y, s)\left(\frac{y-b}{s-b}\right)^{\lambda} E_{1}\left[c_{1}(x), \psi(x, s)\right] d s,
$$

where

$$
\psi(x, y)=E_{4}\left[c_{6}(y), c_{7}(y), f(x, y)\right]-\frac{1}{\beta} \int_{a}^{x} \Gamma_{4}(x, t)\left(\frac{x-a}{t-a}\right)^{\alpha} E_{4}\left[c_{6}(y), c_{7}(y), f(t, y)\right] d t .
$$

Thus, the following theorem takes place.

Theorem 3. We assume that the following conditions are fulfilled:

1) $G(x, y)=A(x) E(y), H(x, y)=B(x) E(y)$ in an I-D equation (1);

2) The roots of the characteristic equations (10) and (11) are such that $\lambda=-E(b)>0, \operatorname{Re} \mu_{1,2}=\alpha>1$;

3) The following inclusions take place

$$
\begin{aligned}
& A(x) \in \mathbb{C}_{A(a)}^{\varepsilon}\left(\overline{\Gamma_{1}}\right), B(x) \in \mathbb{C}_{B(a)}^{\varepsilon}\left(\overline{\Gamma_{1}}\right), E(y) \in \mathbb{C}_{E(b)}^{\varepsilon}\left(\overline{\Gamma_{2}}\right), \\
& f(x, y) \in \mathbb{C}^{\alpha-1, \lambda-1}(\bar{D}),\left\{c_{6}(y), c_{7}(y)\right\} \in \mathbb{C}^{\lambda-1}\left(\overline{\Gamma_{2}}\right) .
\end{aligned}
$$

Then the solution of equation (1) in the class $C_{x}^{1,0}(\bar{D})$ can be represented by formula (18).

Remark 2. If $\lambda=-E(b)<0, \alpha<1$, we obtain the unique solution of equation (1) in the form

$$
\varphi(x, y)=E_{1}[0, \psi(x, y)]-\int_{b}^{y} \Gamma_{1}(y, s)\left(\frac{y-b}{s-b}\right)^{\lambda} E_{1}[0, \psi(x, s)] d s,
$$

where

$$
\psi(x, y)=E_{4}[0,0, f(x, y)]-\int_{a}^{x} \Gamma_{4}(x, t)\left(\frac{x-a}{t-a}\right)^{\alpha} E_{4}[0,0, f(t, y)] d t .
$$

\section{Boundary value problem}

One of the most important results in the theory of I-D equations is solving the Cauchy type problem and boundary value problems. The Cauchy type problem, when $x_{0}$ does not the same as singular point, is solved as in ordinary theory. But in the theory of singular I-D equations it is interesting when corresponding conditions are given at singular point. In this case such kind problems we call singular Cauchy type problems. The singular Cauchy type problems are different from ordinary Cauchy problems, that in our case the problem gives with some weights.

We introduce the following denotation:

$$
\left\{\begin{array}{l}
P_{b, \lambda}^{y}[\varphi(x, y)]=\frac{1}{(y-b)^{\lambda}} \varphi(x, y), \\
P_{a, \mu_{1}}^{x}[\varphi(x, y)]=\frac{1}{(x-a)^{\mu_{1}}}\left[\Pi_{b, E(y)}^{y, 1} \varphi(x, y)\right], \\
P_{a, \mu_{1}, \mu_{2}}^{x}\left[\varphi_{x}^{\prime}(x, y)\right]=\frac{1}{(x-a)^{\mu_{1}-\mu_{2}-1}} \frac{d}{d x}\left[\frac{1}{(x-a)^{\mu_{1}}} \Pi_{b, E(y)}^{y, 1} \varphi(x, y)\right] .
\end{array}\right.
$$


Then the following remark holds:

Remark 3. The solution of type (16) has the following property:

$$
\left\{\begin{array}{l}
{\left[P_{b, \lambda}^{y}[\varphi(x, y)]\right]_{y=b}=c_{1}(x)} \\
{\left[P_{a, \mu_{1}}^{x}[\varphi(x, y)]\right]_{x=a}=c_{2}(y)} \\
{\left[P_{a, \mu_{1}, \mu_{2}}^{x}\left[\varphi_{x}^{\prime}(x, y)\right]\right]_{x=a}=\left(\mu_{2}-\mu_{1}\right) c_{3}(y)}
\end{array}\right.
$$

Now we consider the following singular Cauchy type problem:

Singular Cauchy type problem. Let the roots of characteristic equations (10) and (11) be such that $\lambda=-E(b)>0,1<\mu_{1}<\mu_{2}$. It is required to find such solution of equation (1), which belongs to the class $C_{x}^{1,0}(\bar{D})$ and satisfies the following initial value conditions:

$$
\left\{\begin{array}{l}
{\left[P_{b, \lambda}^{y}[\varphi(x, y)]\right]_{y=b}=\omega_{1}(x)} \\
{\left[P_{a, \mu_{1}}^{x}[\varphi(x, y)]\right]_{x=a}=\omega_{2}(y)} \\
{\left[P_{a, \mu_{1}, \mu_{2}}^{x}\left[\varphi_{x}^{\prime}(x, y)\right]\right]_{x=a}=\omega_{3}(y)}
\end{array}\right.
$$

where $\omega_{1}(x), \omega_{2}(y), \omega_{3}(y)$ are given functions on the domain $D$.

By virtue of integral representation (16) and its property (19), for equation (1) we find functions $c_{1}(x), c_{2}(y)$, $c_{3}(y)$ by means of the given functions $\omega_{1}(x), \omega_{2}(y), \omega_{3}(y): c_{1}(x)=\omega_{1}(x), c_{2}(y)=\omega_{2}(y), c_{3}(y)=\frac{1}{\mu_{2}-\mu_{1}} \omega_{3}(y)$. Therefore substituting these values into (16), the unique solution of the singular Cauchy type problem be found as

$$
\left\{\begin{array}{l}
\varphi(x, y)=E_{1}\left[\omega_{1}(x), \psi(x, y)\right]-\int_{b}^{y} \Gamma_{1}(y, s)\left(\frac{y-b}{s-b}\right)^{\lambda} E_{1}\left[\omega_{1}(x), \psi(x, s)\right] d s \\
\psi(x, y)=E_{2}\left[\omega_{2}(y), \frac{1}{\mu_{2}-\mu_{1}} \omega_{3}(y), f(x, y)\right]- \\
-\frac{1}{\sqrt{D_{1}}} \int_{a}^{x} \Gamma_{2}(x, t)\left(\frac{x-a}{t-a}\right)^{\mu_{1}} E_{2}\left[\omega_{2}(y), \frac{1}{\mu_{2}-\mu_{1}} \omega_{3}(y), f(t, y)\right] d t
\end{array}\right.
$$

So we have proved the following theorem.

Theorem. 4 Let all conditions of Theorem 1 be fulfilled. Then the singular Cauchy type problem has a unique solution, which is given by (20).

Such kind of problems can be investigated, when the roots of characteristic equation (11) are real, equal, complex and conjugate.

\section{References}

1 Assis A.S. The AK Transform / A.S. Assis, S. Kuhn, J. Limaco // Applied Mathematics. - 2017. - 8. - P. 145-153. http://doi.org/10.4236/am.2017.82012

2 Biazar J. Galerkin RBF for Integro-differential equations / J. Biazar, M.A. Asadi // British Journal of Mathematical and Computer Sciences. - 2015. - 11. - № 2. - P. 1-9.

3 Hayani W.A. Solving n-th order Inteqro-differential equations using the combined Laplace TransformAdomain Decomposition Method. / W.A. Hayani // Applied Mathematics. - 2013. - 4. - P. 882-886.

4 Khairullina L.E. Uniform wavelet-approximation of singular integral equation solutions / L.E. Khairullina, A.V. Ozhegova // Lobachevskii Journal of Mathematics. - 2018. — 39. — № 6. - P. 777-782.

5 Khalifa A.S. Approximate Solution of Lane-Emden type Equations Using Variation of Parameters Method with an Auxiliary Parameters / A.S. Khalifa, H.N. Hassan // Journal of Applied Mathematics and Physics. - 2019. - 7. - P. 921-941.

6 Supriya M. Solution of Modified Equations of Emden-Type by Differential Transform Method / M. Supriya, R. Banamaly, K.Ch. Pratik // Journal of Modern Physics. - 2011. - 2. - P. 559-563.

7 Асанова А.Т. Численное решение задачи управления для интегро-дифференциальных уравнений / А.Т. Асанова, Э.А. Бакирова, Ж.М. Кадирбаева // Журн. вычисл. матем. и матем. физики. - 2020. - 60. - № 2. - C. 197-215. 
8 Бакирова Э.А. Численный метод решения линейной краевой задачи для интегро-дифференциальных уравнений на основе сплайн-аппроксимации / Э.А. Бакирова, Н.Б. Искакова, А.Т. Асанова // Укр. матем. журн. - 2019. - 71. - № 9. - С. 1176-1191.

9 Белкина Т.А. Сингулярная краевая задача для интегро-дифференциального уравнения в модели страхования со случайными премиями: анализ и численное решение / Т.А. Белкина, Н.Б. Конюхова, С.В. Курочкин // Журн. вычисл. матем. и матем. физики. - 2012. - 52. - № 10. - С. 1384-1416.

10 Федотов А.И. Аппроксимация решений сингулярных интегро-дифференциальных уравнений полиномами Эрмита-Фейера / А.И. Федотов // Уфим. матем. журн. - 2018. - № 2. - С. 109-117.

11 Fomenco A.T. Singularities of integrale Liouville systems, reduction of integrals to lower degree and topological billiards / A.T. Fomenco, V.V. Vedyushkina // Theoretical Applied Mechanics. - 2019. 46. - № 1. - P. 47-63.

12 Бободжанов А.А. Метод нормальных форм в сингулярно-возмущенных системах интегродифференциальных уравнений Фредгольма с быстро изменяющимися ядрами / А.А. Бободжанов, В.Ф. Сафонов // Матем. сб. - 2013. - 204. - № 7. - С. 979-1002.

13 Зарипов С.К. Построение аналога теоремы Фредгольма для одного класса модельных интегродифференциальных уравнений первого порядка с сингулярной точкой в ядре / С.К. Зарипов // Вестн. Том. гос. ун-та. Математика и механика. - 2017. - 46. - С. 24-35.

14 Зарипов С.К. Построение аналога теоремы Фредгольма для одного класса модельных интегродифференциальных уравнений первого порядка с логарифмической особенностью в ядре / C.K. Зарипов // Вестн. Самар. гос. техн. ун-та. Сер. физ.-матем. науки. - 2017. - 21. - № 2. C. $236-248$.

15 Зарипов С.К. Об одной новой методике решения одного класса модельных интегро-дифференциальных уравнений первого порядка с сингулярным ядром / C.К. Зарипов // Математическая физика и компьютерное моделирование. - 2017. - 20. - № 4. - С. 68-75.

16 Hamoud A.A. Existence and Uniqueness of the Solution for Volterra-Fredholm Integro-Differential Equations / A.A. Hamoud, K.P. Ghadle // Journal of Siberian Federal University. Mathematics and Physics. - 2018. - 11. - № 6. - P. 692-701.

17 Laurencot Ph. Some singular equations modeling MEMS / Ph. Laurencot, Ch. Walker // Bulletin of the American Mathematical Society. - 2017. - 54. - № 3. - P. 437-479.

18 Асхабов С.Н. Сингулярные интегро-дифференциальные уравнения с ядром Гильберта и монотонной нелинейностью / С.Н. Асхабов // Владикавказ. матем. журн. - 2017. - 19. - № 3. - С. 11-20.

19 Фалалаев М.В. Сингулярные интегро-дифференциальные уравнения специального вида в банаховых пространствах и их приложения / М.В.Фалалаев // Изв. Иркут. гос. ун-та. Сер. матем. - 2013. - 6. - № 4. - C. 128-137.

20 Falaleev M.V. Fundamental operator-valued functions of singular integrodifferential operators in Banach spaces / M.V. Falaleev // Journal of Mathematical Sciences. - 2018. - 230. — № 5. - P. 782-785.

21 Sidorov N.A. Continuous and generalized solutions of singular integro-differential equations in Banach spaces / N.A. Sidorov, M.V. Falaleev // Bulleten of South Ural State University. Mathematical modelling and Programming. - 2012. - № 11. - P. 62-74.

22 Yuldashev T.K. On inverse boundary value problem for a Fredholm integro-differential equation with degenerate kernel and spectral parameter / T.K. Yuldashev // Lobachevskii Journal of Mathematics. 2019. - 40. - № 2. - Р. 230-239.

23 Юлдашев Т.К. Об одной нелокальной краевой задаче для нелинейного интегро-дифференциального уравнения Фредгольма с вырождением ядра / Т.К. Юлдашев // Дифференц. уравнения. - 2018. 54. - № 12.- C. 1687-1694.

24 Abubakirov N.R. Classes of finite solutions to the inverse problem of the logarithmic potential / N.R. Abubakirov, L.A. Aksentev // Lobachevskii Journal of Mathematics. - 2018. - 39. - № 2. - P. 151-160.

25 Gowers W.T. Generalization of Fourier analysis and how to apply them / W.T. Gowers // Bulletin of the American Mathematical Society. - 2017. - 54. - № 1. - P. 1-44.

26 González-Gaxiola O. An $\alpha$-Mellin transform and some of its applications / O. González-Gaxiola, J.A. Santiago // International Journal of Contemprorary Mathematical Sciences. - 2012 . — 7. — № 48. - P. 23532361. 
27 Al-Omari S.K.Q. On the distributional Mellin transformation and its extension to Boehmian spaces / S.K.Q. Al-Omari // International Journal of Contemprorary Mathematical Sciences. — 2011. — 6. № 17. - P. 801-810.

28 Yuldashev T.K. New type super singular integro-differential equation and its conjugate equation / T.K. Yuldashev, S.K. Zarifzoda // Lobachevskii Journal of Mathematics. - 2020. — 41. - № 6 . P. 1123-1130.

29 Yuldashev T.K. Mellin transform and integro-differential equations with logarithmic singularity in the kernel / T.K. Yuldashev, S.K. Zarifzoda // Lobachevskii Journal of Mathematics. — 2020. — 41. — № 9. - P. 1910-1917.

T.K. Юлдашев, С.К. Зарифзода

\title{
Сингулярлық интегро-дифференциалдық теңдеулердің бір жаңа класы туралы
}

\begin{abstract}
Мақалада ядрода ерекшелігі бар модельдік және модельдік емес дербес туындылы интегродифференциалдық теңдеулердің жаңа класы үшін еркін функциялар көмегімен интегралдық үйір түріндегі шешімі алынған. Интегро-дифференциалдық теңдеулердің бұл түрі Коши типтес сингулярлық интегро-дифференциалдық теңдеулерден өзгеше. Коши типіндегі сингулярлық интегродифференциалдық теңдеулер аналитикалық функциялар теориясының әдістерімен қарастырылған. Зерттеу барысында сингулярлық интегро-дифференциалдық операторлардың жаңа түрлері енгізіліп, осы енгізілген операторлардың негізгі қасиеттері зерттелді. Зерттелетін теңдеудің шешімі х және уке қатысты екі теңдеу жүйесінің шешіміне тең екендігі көрсетілген, олардың бірі - интегралдық теңдеу, ал екіншісі - интегро-дифференциалдық теңдеу болып табылады. Әрі қарай модельдік емес интегро-дифференциалдық теңдеулер регуляризациялау әдісімен зерттелген. Модельдік емес теңдеуді реттеудің бұл әдісі теңдеудің модельдік бөлігін таңдауға және талдауға негізделген және ядродағы әлсіз ерекшелігі бар екінші типтегі Вольтерр типтес екі интегралдық теңдеуді шешуге дейін азаяды. Теңдеуде модельдік емес бөліктің болуы шешімдердің жалпы құрылымына әсер етпейтіні көрсетілген. Сондықтан интегро-дифференциалдық теңдеулердің осы класы үшін модельдік теңдеулерді зерттеу маңызды. Осы интегро-дифференциалдық теңдеудің шешімі кез келген ерікті функциялардан тәуелді болған жағдайда Коши типтес есептер зерттеледі.
\end{abstract}

Kiлm сөздер: сингулярлық интегро-дифференциалдық теңдеу, модельдік теңдеу, модельдік емес теңдеу, сипаттамалық теңдеу, Коши типтес есеп.

Т.К. Юлдашев, С.К. Зарифзода

\section{Об одном новом классе сингулярных интегро-дифференциальных уравнений}

В статье для нового класса модельных и немодельных интегро-дифференциальных уравнений в частных производных с особенностью в ядре получено решение в виде семейства интегрального представления с помощью произвольных функций. Такой тип интегро-дифференциальных уравнений отличается от сингулярных интегро-дифференциальных уравнений типа Коши. Сингулярные интегро-дифференциальные уравнения типа Коши изучаются методами теории аналитических функций. В процессе нашего исследования введены новые типы сингулярных интегро-дифференциальных операторов и изучены основные свойства этих введенных операторов. Показано, что решение исследуемого уравнения эквивалентно решению системы двух уравнений относительно $x$ и $y$, одно из которых является интегральным уравнением, а другое - интегро-дифференциальным уравнением. Далее немодельные интегро-дифференциальные уравнения исследованы методом регуляризации. Этот метод регуляризации немодельного уравнения основан на выборе и анализе модельной части уравнения и сведен к решению двух интегральных уравнений типа Вольтерра второго рода со слабой особенностью в ядре. Показано, что наличие немодельной части в уравнении не влияет на общую 
структуру решений. Отсюда важное значение приобретает исследование модельных уравнений для данного класса интегро-дифференциальных уравнений. В случаях, когда решение данного интегродифференциального уравнения зависит от любых произвольных функций, исследованы задачи типа Коши.

Ключевые слова: сингулярное интегро-дифференциальное уравнение, модельное уравнение, немодельное уравнение, характеристическое уравнение, задача типа Коши.

\section{References}

1 Assis, A.S., Kuhn, S., \& Limaco, J. (2017). The AK Transform. Applied Mathematics, 8, 145-153.

2 Biazar, J., \& Asadi, M.A. (2015). Galerkin RBF for Integro-differential equations. British Journal of Mathematics and Computer Sciences, 11(2), 1-9.

3 Hayani, W.A. (2013). Solving n-th order Inteqro-differential equations using the combined Laplace Transform-Adomain Decomposition Method. Applied Mathematics, 4, 882-886.

4 Khairullina, L.E., \& Ozhegova, A.V. (2018). Uniform wavelet-approximation of singular integral equation solutions. Lobachevskii Journal of Mathematics, 39(6), 777-782.

5 Khalifa, A.S., \& Hassan, H.N. (2019). Approximate Solution of Lane-Emden type Equations Using Variation of Parameters Method with an Auxiliary Parameters. Journal of Applied Mathematics and Physics, 7, 921-941.

6 Supriya, M., Banamaly, R., \& Pratik, K.Ch. (2011). Solution of Modified Equations of Emden-Type by Differential Transform Method. Journal of Modern Rhysics, 2, 559-563.

7 Assanova, A.T., Bakirova, E.A., \& Kadirbayeva, Z.M. (2020). Chislennoe reshenie zadachi upravleniia dlia intehrodifferentsialnykh uravnenii [Numerical Solution to a Control Problem for Integro-Differential Equations]. Zhurnal vychislitelnoi matematiki i matematicheskoi fiziki - Computational Mathematics and Mathematical Physics, 60(2), 203-221 [in Russian].

8 Bakirova, E.A., Iskakova, N.B., \& Assanova, A.T. (2020). Chislennyi metod resheniia lineinoi kraevoi zadachi dlia intehro-differentsialnykh uravnenii na osnove splain-approksimatsii [Numerical Method for the Solution of Linear Boundary-Value Problems for Integrodifferential Equations Based on Spline Approximations. Ukrainskii matematicheskii zhurnal - Ukranian Mathematical Journal, 71(9), 1341-1358 [in Russian].

9 Belkina, T.A., Konyukhova, N.B., \& Kurochkin, S.V. (2012). Sinhuliarnaia kraevaia zadacha dlia intehrodifferentsialnoho uravneniia $\mathrm{v}$ modeli strakhovaniia so sluchainymi premiiami: analiz i chislennoe reshenie [Singular boundary value problem for an integro-differential equation in a random premium insurance model: analysis and numerical solution]. Zhurnal vychislitelnoi matematiki i matem. fiziki - Computational Mathematics and Mathematical Physics, 52(10), 1384-1416 [in Russian].

10 Fedotov, A.I. (2018). Approksimatsiia reshenii sinhuliarnykh intehro-differentsialnykh uravnenii polinomami Ermita-Feiera [Approximation of solutions to singular integro-differential equation by Hermite-Fejer polynomials]. Ufimskii matematicheskii zhurnal - Ufa Mathematical Journal, 2, 109-117 [in Russian].

11 Fomenco, A.T., \& Vedyushkina, V.V. (2019). Singularities of integrale Liouville systems, reduction of integrals to lower degree and topological billiards. Theoretical and Applied Mechanics, 46(1), 47-63.

12 Bobodzhanov, A.A., \& Safonov, V.F. (2013). Metod normalnykh form v sinhuliarno-vozmushchennykh sistemakh intehro-differentsialnykh uravnenii Fredholma s bystro izmeniaiushchimisia yadrami [The method of normal form in singularly perturbed systems of Fredholm type integro-differential equations with quickly changing kernels]. Matematicheskii sbornik - Sbornik: Mathematics, 204(7), 979-1002 [in Russian].

13 Zaripov, S.K. (2017). Postroenie analoha teoremy Fredholma dlia odnoho klassa modelnykh intehrodifferentsialnykh uravnenii pervoho poriadka s sinhuliarnoi tochkoi v yadre [Construction of an analog of the Fredholm theorem for a class of model first order integro-differential equations with a singular point in the kernel]. Vestnik Tomskoho hosudarstvennoho universiteta. Matematika i mekhanika - Tomsk State University Journal of Mathematics and Mechanics, 46, 24-35 [in Russian].

14 Zaripov, S.K. (2017). Postroenie analoha teoremy Fredholma dlia odnoho klassa modelnykh intehrodifferentsialnykh uravnenii pervoho poriadka s loharifmicheskoi osobennostiu v yadre [A construction of analog of Fredgolm theorems for one class of first order model integro-differential equation with 
logarithmic singularity in the kernel]. Vestnik Samarskoho hosudarstvennoho tekhnicheskoho universiteta. Seria Fiziko-matematicheskie nauki - Journal Samara State Technical University, Seria Physical and Mathematical Sciences, 21(2), 236-248 [in Russian].

15 Zaripov, S.K. (2017). Ob odnoi novoi metodike resheniia odnoho klassa modelnykh intehro-differentsialnykh uravnenii pervoho poriadka s sinhuliarnym yadrom [On a new method of solving of one class of model first-order integro-differential equations with singularity in the kernel]. Matematicheskaia fizika $i$ kompiuternoe modelirovanie - Matheematical Physics and Computer Modeling, 20(4), 68-75 [in Russian].

16 Hamoud, A.A., \& Ghadle, K.P. (2018). Existence and Uniqueness of the Solution for Volterra-Fredholm Integro-Differential Equations. Journal of Siberian Federal University. Mathematics \&s Physics, 11(6), 692-701.

17 Laurencot, Ph., \& Walker, Ch. (2017). Some singular equations modeling MEMS. Bulletin of the American Mathematical Society, 54(3), 437-479.

18 Askhabov, S.N. (2017). Sinhuliarnye intehro-differentsialnye uravneniia s yadrom Hilberta i monotonnoi nelineinostiu [Singular integro-differential equations with Hilbert kernel and monotone nonlinearity]. Vladikavkazskii matematicheskii zhurnal - Vladikavkaz Mathematical Journal, 19(3), 11-20 [in Russian].

19 Falaleev, M.V. (2013). Sinhuliarnye intehro-differentsialnye uravneniia spetsialnoho vida v banakhovykh prostranstvakh i ikh prilozheniia [Singular integro-differential equations of the special type in Banach spaces and it's applications]. Izvestiia Irkutskoho hosudarstvennoho universiteta. Seria Matematika The Bulletin of Irkutsk State University. Series Mathematics, 6(4), 128-137 [in Russian].

20 Falaleev, M.V. (2018). Fundamental operator-valued functions of singular integrodifferential operators in Banach spaces. Journal of Mathematical Sciences, 230(5), 782-785.

21 Sidorov, N.A., \& Falaleev, M.V. (2012). Continuous and generalized solutions of singular integro-differential equations in Banach spaces. Bulleten of South Ural State University. Mathematical modelling and Programming, 11, 62-74.

22 Yuldashev, T.K. (2019). On inverse boundary value problem for a Fredholm integro-differential equation with degenerate kernel and spectral parameter. Lobachevskii Journal of Mathematics, 40(2), 230-239.

23 Yuldashev, T.K. (2018). Ob odnoi nelokalnoi kraevoi zadache dlia nelineinoho intehro-differentsialnoho uravneniia Fredholma s vyrozhdeniem yadra [Nonlocal boundary value problem for a nonlinear Fredholm integro-differential equation with degenerate kernel. Differentsialnye uravneniia - Differential Equations, 54(12), 1646-1653 [in Russian].

24 Abubakirov, N.R., \& Aksentev, L.A. (2018). Classes of finite solutions to the inverse problem of the logarithmic potential. Lobachevskii Journal of Mathematics, 39(2), 151-160.

25 Gowers, W.T. (2017). Generalization of Fourier analysis and how to apply them. Bulletin of the American Mathematical Society, 54(1), 1-44.

26 González-Gaxiola, O., \& Santiago J.A. (2012). An $\alpha$-Mellin transform and some of its applications. International Journal of Contemprorary Mathematical Sciences, 7, 48, 2353-2361.

27 Al-Omari, S.K.Q. (2011). On the distributional Mellin transformation and its extension to Boehmian spaces. International Journal of Contemprorary Mathematical Sciences, 6, 17, 801-810.

28 Yuldashev, T.K., \& Zarifzoda S.K. (2020). New type super singular integro-differential equation and its conjugate equation. Lobachevskii Journal of Mathematics, 41(6), 1123-1130.

29 Yuldashev, T.K., \& Zarifzoda, S.K. (2020). Mellin transform and integro-differential equations with logarithmic singularity in the kernel. Lobachevskii Journal of Mathematics, 41(9), 1910-1917. 


\section{АВТОРЛАР ТУРАЛЫ МӘЛІМЕТТЕР СВЕДЕНИЯ ОБ АВТОРАХ INFORMATION ABOUT AUTHORS}

Abdul Rahman, N.A. - Doctor of Mathematical Sciences, Professor, School of Mathematical Sciences, Universiti Sains Malaysia (USM), Penang, Malaysia.

Abdullah, D.Kh. - M.Sc., Assistant Lecture of the Department of Mathematics, College of Science, University of Sulaimani, Kurdistan Region, Sulaimani, Iraq.

Ahmed, Sh.Sh. - PhD, Assistant Professor of the Department of Mathematics, College of Science, University of Sulaimani, Kurdistan Region, Sulaimani, Iraq.

Attaev, A.Kh. - Candidate of Physical and Mathematical Sciences, Docent, Head of department computeraided design mixed systems and control, Institute of Applied Mathematics and Automation of KabardinBalkar Scientific Centre of the Russian academy of sciences, Nalchik, Russia.

Bekbolat, B. - Master of Science, Associate Researcher, Al-Farabi Kazakh National University, Institute of Mathematics and Mathematical Modeling, Almaty, Kazakhstan and Ghent University, Ghent, Belgium.

Bokayev, N.A. - Professor, Doctor of Physical and Mathematical Sciences, L.N. Gumilyov Eurasian National University, Nur-Sultan, Kazakhstan.

Davoodi, S.M. - PhD, Candidate of Mathematical Sciences, School of Mathematical Sciences, Universiti Sains Malaysia (USM), Penang, Malaysia.

Iskakov, S.A. - PhD, E.A. Buketov Karaganda University, Karaganda, Kazakhstan.

Islomov, B.I. - Professor, Doctor of Physical and Mathematical Sciences, Professor of the Department of Differential Equations and Mathematical Physics, National University of Uzbekistan, Tashkent, Uzbekistan.

Jenaliyev, M.T. - Doctor of Physical and Mathematical Sciences, Professor, Chief Scientific Researcher, Institute of Mathematics and Mathematical Modeling, Almaty, Kazakhstan.

Jwamer, K.H.F. - PhD, Professor of the Department of Mathematics, College of Science, University of Sulaimani, Kurdistan Region, Sulaimani, Iraq.

Kasymova, L.Zh. - PhD student, E.A. Buketov Karaganda University, Karaganda Technical University, Karaganda, Kazakhstan.

Khairkulova, A.A. - Master of mathematics, PhD student, L.N. Gumilyov Eurasian National University, Nur-Sultan, Kazakhstan.

Kosmakova, M.T. - PhD, E.A.Buketov Karaganda University, Karaganda, Kazakhstan.

Kvasnikov, V.P. - Professor, Doctor of Technical Sciences, Head of the Department of Computerized Electrical Systems and Technologies of the National Aviation University, Kyiv, Ukraine.

Muratbekov, M.B. - Doctor of Physical and Mathematical Science, Professor, Taraz Regional University named after M.Kh.Dulaty, Taraz, Kazakhstan.

Mussina, N.M. - PhD student, E.A. Buketov Karaganda University, Karaganda, Kazakhstan.

Omarova, M.T. - PhD student, E.A. Buketov Karaganda University, Karaganda, Kazakhstan. 
Ospanov, M.N. - Candidate of physical and mathematical sciences, Docent, Head of the Higher mathematics department, L.N.Gumilyov Eurasian National University, Nur-Sultan, Kazakhstan.

Ramazanov, M.I. - Doctor of Physical and Mathematical Sciences, Professor, Professor of Chair «Mathematical Analysis and Differential Equations», E.A. Buketov Karaganda University, Karaganda, Kazakhstan.

Shkvarnytska, T.Y. - Candidate of Technical Sciences, Associate Professor of the Department of Computerized Electrical Systems and Technologies of the National Aviation University, Kyiv, Ukraine.

Suleimbekova, A.O. - Doctoral student, L.N. Gumilyev Eurasian National University, Nur-Sultan, Kazakhstan.

Tanin, A.O. - PhD student, E.A. Buketov Karaganda University, Karaganda, Kazakhstan.

Tleubergenov, M.I. - Doctor of Physical and Mathematical Sciences, Professor, Chief Researcher, Institute of Mathematics and Mathematical Modeling and Al-Farabi Kazakh National University, Almaty, Kazakhstan.

Tokmagambetov, N. - PhD in Mathematics, Associate Professor, Al-Farabi Kazakh National University, Institute of Mathematics and Mathematical Modeling, Almaty, Kazakhstan and Ghent University, Ghent, Belgium.

Tuzelbaeva, G. - Master student, Institute of Mathematics and Mathematical Modeling amd Al-Farabi Kazakh National University, Almaty, Kazakhstan.

Ubaydullaev, U.Sh. - Doctoral student, National University of Uzbekistan, Tashkent, Uzbekistan.

Vassilina, G.K. - PhD, Senior Researcher, Institute of Mathematics and Mathematical Modeling and Almaty University of Power Engineering and Telecommunications named after G.Daukeev, Almaty, Kazakhstan.

Yehorov, S.V. - Candidate of Technical Sciences, Assistant of the Department of Computerized Information Protection Systems of the National Aviation University, Kyiv, Ukraine.

Yesbayev, A.N. - PhD student, L.N.Gumilyov Eurasian National University, Nur-Sultan, Kazakhstan.

Yeshkeyev, A.R. - Doctor of physical and mathematical sciences, Head of the Department of Algebra, Mathematical Logic and Geometry named after professor T.G. Mustafin, Professor of E.A. Buketov Karaganda University, Karaganda, Kazakhstan.

Yuldashev, T.K. - Candidate of Physical and Mathematical Sciences, Docent, Associate Professor, National University of Uzbekistan, Tashkent, Uzbekistan.

Zarifzoda, S.K. - Candidate of Physical and Mathematical Sciences, Docent, Associate Professor, Tajik National University, Dushanbe, Tajikistan. 